
OAK ST. HDSF

\section{THE UNIVERSITY}

\section{OF ILLINOIS}

\section{LIBRARY}
195
V66Yc2 
Return this book on or before the Latest Date stamped below. A charge is made on all overdue books.

University of Illinois Library

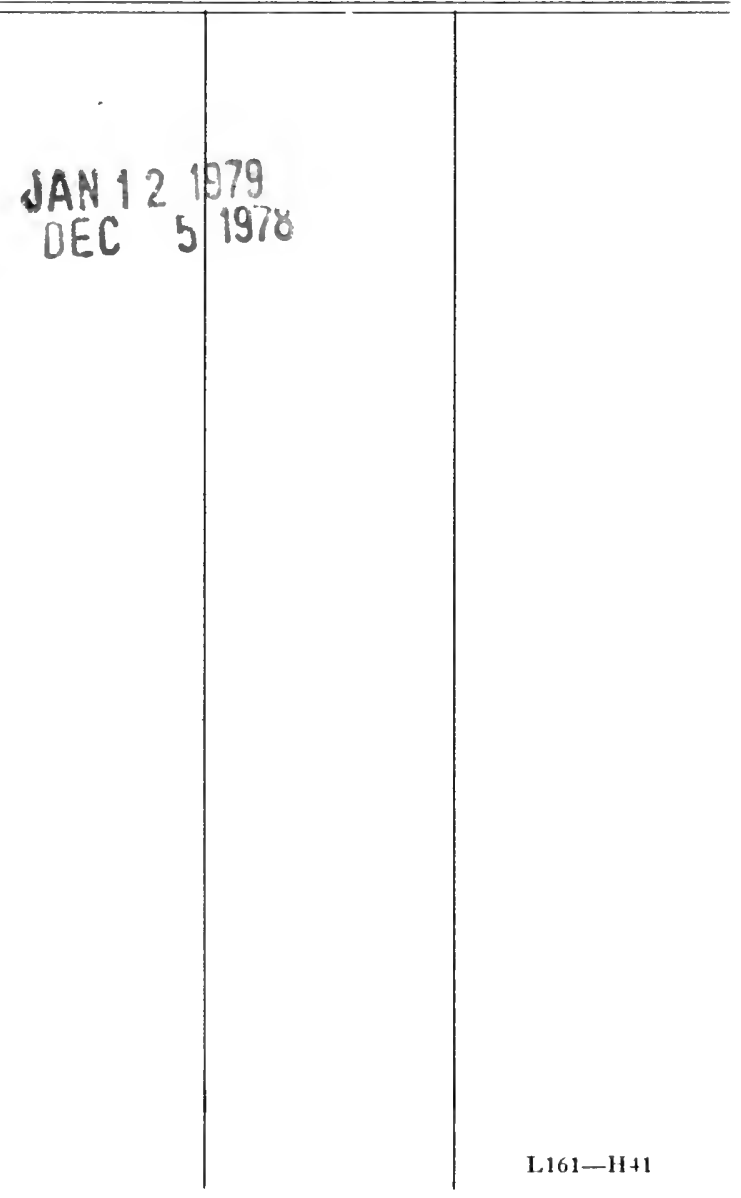





\section{SAGGI FILOSOFICI}

II

VICO 


\section{BENEDETTO CROCE}

\section{LA FILOSOFIA}

DI

\section{GIAMBATTISTA VICO}

Seconda edizione RIveduta

BARI

GIUS. LATERZA \& FIGLI

TIYOGRAFI-RDITORI-LIBBAI

1922 
PROPRIETA LETTERARIA

A NORMA DErLE VIGENTI LEGGI 


$$
\begin{aligned}
& 195 \\
& V 66 \text { Yc } 2
\end{aligned}
$$

8

$\Lambda$

GUGLIELMO WINDELBAND

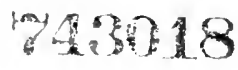





\section{AVVERTENZA}

Per quali ragioni a me sia sembrata necessaria una nuova esposizione della filosofia del Vico, potrà agevolmente desumersi dai cenni sulla fortuna di questo scrittore e dalle notizic bibliografiche, che si leggono nella seconda e terza appendice del presente volume.

Qui occorre avvertire soltanto che la mia esposizione non vuol essere un riassunto libro per libro e parte per parte degli scritti del Vico; e, anzi, presup. pone la conoscenza di questi scritti e, ove manchi, vuol eccitare il lettore a procacciarsela per meglio seguire, e per riscontrare, le interpetrazioni e i giudizî che gli vengono da me offerti.

Su questo presupposto, pur valendomi assai spesso (specialmente nei capitoli relativi alla storiografia) delle parole testuali dell'autore, non ho creduto opportuno virgoleggiarle (salvo dove mi piacesse dare risalto alla precisa espressione originale), perché, avendole di solito combinate da brani sparsi nei piủ varî luoghi e ora abbreviate ora allargate e sempre fram- 
mischiate liberamente con parole e frasi mie di commento, il continuo virgoleggiarle sarebbe stato un mettere in mostra, con piủ di fastidio che di utilita, il rovescio del mio ricamo, che ciascuno potrá osservare da sé, quando ne abbia voglia, col sussidio dei rimandi che ho messi in fondo al libro.

Desideroso di attestare, per quanto mi era possibile, in ogni particolare del mio lavoro, la reverenza che si deve al gran nome del Vico, mi sono studiato di essere breve, di quella brevità che egli considerava quasi suggello di libri scientifici ben meditati. Al qual uopo ho sacrificato anche le discussioni coi singoli interpetri, contentandomi di semplici accenni. Del resto, parte delle interpetrazioni qui esposte mi sembrano frutti maturi delle indagini e controversie che costituiscono la migliore letteratura vichiana; e tutta quell'altra parte, che è mia personale, e l'idea stessa generale del mio libro, difenderò a suo tempo, se sará il caso, contro i dissenzienti e gli obiettanti, nel modo diretto che nel corso dell'esposizione non ho stimato di adoperare. Perché io spero che questo libro avrà l'effetto non già di spegnere ma di raccendere le discussioni intorno alla filosofia del Vico: di questo Altvater, come lo chiamó il Goethe, che $\dot{e}$ fortuna per un popolo possedere, e al quale bisognerà sempre fare capo per sentile italianamente la moderna filosofia, pur pensandola cosmopoliticamente.

La dedica del mio lavoro (oltre a essere omaggio a uno dei maggiori masstri odierni della storia della filosofia) vuol esprimere l'augurio e la speranza che 
venga presto riempita, in tale storia, la lacuna, sulla quale ho richiamato l'attenzione piú volte, e specialmente alla fine della seconda delle appendici di questo volume.

Raiano (Aquila), settembre 1910.

L'augurio espresso nelle ultime linee della precedente avvertenza ebbe compimento, e non solo il Windelband diè luogo alla filosofia del Vico nella quinta edizione della sua Storia della filosofia moderna (Leipzig, 1911, I, 597-98), ma il mio libro fu subito tradotto in inglese e in francese, e altre versioni se ne preparavano, e fiorivano le indagini e le discussioni, quando la guerra sopravvenne a sospendere quella ripresa di studî e la divulgazione dell'opera vichiana fuori d'Italia. Non sí per altro che, durante la guerra e in relazione ad essa, $i$ concetti vichiani non fossero qua e lá richiamati per dominare col pensiero il corso delle cose; e li richiamó, tra gli altri, lo stesso Windelband, nell'ultimo suo scritto, che fu una « lezione di guerra »sulla Filosofia della storia.

Questa nuova edizione contiene piccole correzioni, schiarimenti e aggiunte, ed è messa al corrente nella. parte bibliografica. La tavola dei rinvii ai testi vichiani è stata resa piủ precisa, e in ció, come nella revisione generale, ho avuto l'amichevole aiuto del Nicolini, benemerito editore della Scienza nuova.

Circa la concezione e il metodo del libro non ho alcun cangiamento da introdurre né pentimento da 
manifestare: sebbene da piủ parti mi sia stata rivolta la facile ma superficialissima critica, che l'interpretazione del Vico vi sia tutta compenetrata dal mio proprio pensiero filosofico, e perciò non sia « oggettiva ». In veritá, chi voglia conoscere davvero il Vico deve leggere e meditare i libri del Vico; e questo è indispensabile, e questa è la sola oggettività possibile: non la cosiddetta « esposizione oggettiva 》 che altri ne faccia, e che non potrebbe riuscire se non lavoro estrinseco e materiale. L'esposizione, invece, storica e critica di un filosofo ha una diversa e piú alta oggettivitá, ed è necessariamente il dialogo tra un antico e un nuovo pensiero, nel quale solamente l'antico pensiero viene inteso e compreso. E tale $\dot{e}$, o procura di essere, il mio libro. Che cosa avrei potuto intendere io del Vico, se non mi fossi travagliato su problemi strettamente congiunti ai suoi o derivanti da quelli suoi?

Per questa ragione anche non posso dare importanza all'opposizione che mi è venuta da egregi scrittori cattolici, i quali è naturale che vedano le cose con occhi diversi dai miei. Ciò che, per altro, non mi sembra logico, è il loro sforzo di ridurre il Vico a pensatore ortodosso; nel quale sforzo urtano inevitabilmente in due gravi difficolta. In primo luogo essi vengono a trovarsi di fronte all'impossibilitá di spiegare perché mai il Vico, che, a loro giudizio, non avrebbe fatto altro che ripetere o rinfrescare i concetti della tradizione filosofica cattolica, sia sembrato e sembri tanto originale e rivoluzionario, e sia andato tanto a genio ai pensatori moderni. E parimente, in 
secondo luogo, si tolgono il modo di spiegare l'avversione che per lui provarono gli scrittori cattolici del suo secolo e taluno insigne del secolo seguente, come, per es., Cesare Balbo, che lo sentí estraneo alla scienza cristiana. E questo basti aver detto, perché, riguardoso come credo d'esser sempre stato verso gli scrittori cattolici, non perciò polemizzerei mai con essi, stimando la cosa tanto poco utile, quanto utile e doveroso è, per me, tirare innanzi per la mia via.

Napoli, 27 dicembre 1921.

B. C. 
. 


\section{LA PRIMA Forma della gNoseologia vichiana}

La

a prima forma della dottrina del Vico sulla conoscenza si presenta come diretta critica e antitesi del pensiero cartesiano, che da oltre mezzo secolo dava l'indirizzo generale allo spirito europeo ed era destinato a dominare ancora pel un secolo le menti e gli animi.

Cartesio aveva collocato l'ideale della scienza perfetta nella geometria, sul modello della quale intese a riformare la filosofia e ogni altra parte del sapere. E poiché il metodo geometrico perviene mercé l'analisi a verità intuitive, e da queste muove dipoi per ottenere con deduzione sintetica sempre piú complesse affermazioni, la filosofia, per procedere con rigore di scienza, doveva, a mente di Cartesio, cercare anch'essa il fermo punto d'appoggio in una verita primitiva e intuitiva, dalla quale deducesse tutte le sue ulteriori affermazioni, teologiche, metafisiche, fisiche e morali. L'evidenza, la percezione o idea chiara e distinta era, dunque, criterio supremo; e l'inferenza immediata, l'intuitiva connessione del pensiero con l'essere, del cogito col sum, porgeva la prima verita e la base per la scienza. Con la percezione chiara e distinta, e col dubbio metodico che conduceva al cogito, Cartesio si argomentara 
di sconfiggere una volta per sempre lo scetticismo. Ma, per ciò stesso, tutto quel sapere non ancora ridotto o non riducibile a percezione chiara e distinta e a deduzione geometrica, perdeva ai suoi occhi valore e importanza. Tale la storia, che si fonda sulle testimonianze; l'osservazione naturalistica, non ancora matematizzata; la saggezza pratica e l'eloquenza, che si valgono dell'empirica conoscenza del cuore umano; la poesia, che offre immagini fantastiche. Piuttosto che un sapere, codesti prodotti spirituali erano per Cartesio illusioni e torbide visioni: idee confuse, destinate $o$ a farsi chiare e distinte e percio a svestire la loro anteriore forma d'esistenza, o a trascinare un'esistenza miserabile, indegna dell'attenzione del filosofo. La luce solare del metodo matematico rendeva superfine le fiammelle che sono di guida nelle tenebre e proiettano sovente ombre ingannatrici.

Ora il Vico non si restringe e non si attarda, come altri avversarî di Cartesio, a prendere scandalo per le conseguenze del metodo soggettivo, pericoloso alla religione; o a disputare scolasticamente se il cógito sia o non sia un sillogismo, e se come sillogismo sia o no difettoso; o a protestare con l'offeso buon senso contro il disprezzo cartesiano verso la storia, l'oratoria e la poesia. Egli va diritto al cuore della questione, al criterio stesso stabilito da Cartesio per la verita scientifica, al principio dell'evidenza; e dove il filosofo francese stimava di aver fornito tutto quanto si potesse richiedere per la scienza piú rigorosa, il Vico osserva che, posta l'esigenza alla quale s'intendeva soddisfare, in realtà, col metodo raccomandato, si otteneva ben poco o addirittura nulla.

Bella scienza (dice il Vico) è codesta dell'idea chiara e distinta! Ch' io pensi quel ch'io penso è, sí, cosa indubitabile, ma non mi ha punto l'aria di una proposizione scientifica. Ogni idea, per erronea che sia, può apparire 
evidente; e, non perché a me appaia tale, acquista virtú di scienza. Che "se si pensa, si è anche ", era cosa nota persino al Sosia di Plauto, che esprimeva questa sua persuasione quasi eon le stesse parole della filosofia cartesiana: « sed quom cogito, equidem certo sum ». Ma lo scettico replicherà senupre ai Sosî e ai Cartesî, che egli non dubita di pensare; professera anzi asseverantemente che quel che a lui sembra scorgere è certo, e lo sosterrà con ogni sorta di eavilli; - e che non dubita di essere, anzi cura di esser bene, mereé la sospensione dell'assenso, per non aggiungere ai fastidì delle cose gli altri provenienti dalle opinioni. Ma, nell'affermare cosí, sosterrà insieme che la certezza del suo pensare e del suo essere è coscienza e non scienza; ed è coscienza volgare. Tanto poco la chiara e distinta percezione è scienza, che da quando, per effetto del cartesianismo, essa viene adoperata nella fisica, la conoscenza delle cose naturali non è divenuta punto piú sicura. Cartesio ha spiccato un salto per sollevarsi dalla coscienza volgare alla scienza; ed è rieaduto di piombo in quella coscienza, senza raggiungere la scienza agognata.

Ma in che cosa la verità scientifica consiste, poiché certamente non consiste nella coscienza immediata? In che la scienza differisce dalla semplice coscienza? Qual è il criterio, o, in altri termini, quale la condizione che rende possibile la scienza? Con la chiarezza e con la distinzione non si muove un sol passo; con l'affermazione di un primo vero non si risolve il problema, che non ̀ già cirea un primo vero, ma circa la forma ehe la verita deve avere perché possa essere riconosciuta verita scientifica, ossia verità vera.

Il Vico risponde a questa domanda, e giustifica la sua accusa d'insufficienza al criterio eartesiano, col ricorrere a una proposizione che, a bella prima, potrebbe dirsi ovvia e tradizionale. Tradizionale non in conseguenza della 
tesi storica con la quale il Vico l'accompagna e che egli stesso poi ebbe a rifiutare, cioè che quella proposizione risalga a un'antichissima sapienza italica; ma nel senso che essa era comune e quasi intrinseca al pensiero cristiano. Nulla di piú familiare, infatti, a un cristiano, il quale recita ogni giorno il suo credo in un Dio onnipotente, onnisciente e creatore del cielo e della terra, dell'affermazione che solo Dio può avere scienza piena delle cose, perché egli solo ne è l'autore. Il primo vero (ripete il Vico) è in Dio, perché Dio è il primo fattore; ed è vero infinito perché egli è fattore delle cose tutte, esattissimo perché rappresenta a lui gli elementi cosi esterni come interni delle cose, le quali egli contiene tutte in sé. Questa medesima proposizione circolava nelle scuole, specie, a quanto sembra, presso scotisti e occamisti, e, nel rinascimento, Marsilio Ficino l'asseriva nella Theologia platonica, dicendo che la natura, opera divina, produce le sue cose con vive ragioni dall'intrinseco, come la mente del geometra dall'intrinseco fabbrica le sue figure; e il Cardano ripetera che tale è la vera scienza, la scienza divina, quce res facit, e che di essa tra le umane rende immagine la sola geometria; e lo scettico Sanchez, nel Quod nihil scitur (1581), ricordava che non può a perfecte cognoscere quis qua non creavit, nec Deus creare potuisset nec creata regere quae non perfecte pr(ecognovisset; ipse ergo, solus sapientia, cognitio, intellectus perfectus, omnia penetrat, omnia sapit, omnia cognoscit, omnia intelligit, quia ipse omnia est et in omribus, omniaque ipse sunt et in ipso $\$$. Ma il

$1 \mathrm{Si}$ veda per le origini il mio saggio: Le fonti della gnoseologia vichiana (cit. nell'append. bibliografica), pp. 243-58. Sul concetto del Sanchez (cfr. Opera medica, ed. di Tolosæ T'ectosagum, 1636, p. 110) richiamó l'attenzione il Winderbaxd, Gesch. d. neuer. Philosophie 3, I, p. 23. 
Vico non si restringe ad affermazioni incidentali e, intendendo pel primo la fecondità del concetto espresso in quella proposizione, dall'elogio dell'intinita potenza e sapienza di Dio e dal raffronto con quella limitata dell'uomo ricavò, contro Cartesio, il principio gnoseologico universale, che la condizione per conoscere una cosa è il farla, e il vero è il fatto stesso: "verum ipsum factum».

Non altro che codesto si vuol dire (egli chiarisce), quando si afferma che la scienza è "per çausas scire ", perché la cagione ì quel che per produrre l'effetto non ha bisogno di cosa estranea, è il genere o modo di una cosa: conoscere la cagione è saper mandare ad effetto la cosa, provare dalla causa è farla. In altri termini, è rifare idealmente quel che si è fatto e.si fa praticamente. La cognizione e l'operazione debbono convertirsi tra loro, come in Dio intelletto e volontà si convertono e fanno tutt'uno.

Senonché, stabilito nella connessione del vero e del fatto l'ideale della scienza, e (poiché l'ideale è la vera realtà) conosciuta la natura vera della scienza, la prima conseguenza che da questo riconoscimento deve trarsi è quella stessa che ne traevano i platonici e gli scettici del Rinascimento, l'impossibilità della seienza per l'uomo. Se Dio lia creato le cose, Dio solo le conosce per cause, egli solo ne conosce i generi o modi, ed egli solo ne ha la scienza. Forse che l'uomo ha esso creato il mondo? ha esso creato la propria anima?

All'uomo non è data la scienza, ma la sola coscienza, la quale per l'appunto volge sulle cose di cui non si puó dimostrare il genere o forma oncle si fanno. La verità di coscienza è il lato umano del sapere divino, e sta a questo come la superficie al solido: piuttosto che verita, dovrebbe dirsi certezza. A Dio l'intelligere, all'uomo il solo cogitare, il pensare, l'andare raccogliendo gli elementi delle cose, senza poterli mai raccogliere tutti. A Dio il vero di- 
mostrativo; all'uomo le notizie non dimostrate e non scientifiche, ma o certe per segni indubitati o probabili per forza di buoni raziocinî o verisimili pel sussidio di potenti congetture.

Il certo, la verità di coscienza, non è scienza, ma non perciò è il falso. E il Vico si guarda bene dal chiamare false le dottrinc di Cartesio: egli vuole soltanto degradarle da verità compiute a verità frammentarie, da scicnza a coscienza. Tutt'altro che falso è il cogito ergo sum: il trovarsi finanche sulla bocca del Sosia plautino è argomento non per rigettarlo, anzi per accettarlo, ma come verita di scmplice coscienza. Il pensare, non essendo causa del mio essere, non induce scienza del mio essere; se l'inducesse, essendo l'uomo (secondo che i cartesiani ammettono) mente e corpo, il pensiero sarebbe causa del corpo; il che ci avvolgerebbe tra tutte le spine e gli sterpi delle dispute circa l'azione della mente sul corpo e del corpo sulla mente. Il cogito è, dunque, un mero segno o indizio del mio essere: nient'altro. L' idea chiara e distinta non può darc criterio, non pure delle altre cose ma della mente medesima, perché la mente in quel suo conoscersi non si fa, e, poiché non si fa, ignora il genere o modo onde si conosce. Ma l'idea chiara e distinta è quel che solo è concesso allo spirito dell'uomo, c, come unica ricchezza ch'cgli abbia, preziosissima. Anche per il Vico la metafisica serba il primato fra le scienze umane, che tutte derivano da lei; ma laddove per Cartesio essa può procedere con sicuro metodo di dimostrazione pari a quello geometrico, pel Vico deve contentarsi del probabile, non essendo scienza per cause ma di cause. E del probabile si contentò ai suoi bei tempi, nella Grecia antica e nell' Italia del Rinascimento; e quando volle abbandonare il probabile e si empi la testa dei fumi di quel detto fastoso: « sapientem nihil opinari », cominciò a turbarsi e a decadere. L'esistenza di 
Dio è eerta, ma non è scientificamente dimostrabile, e ogni tentativo di dimostrazione è da considerare documento non tanto di pietà quanto piuttosto d'empietà, perché, per dimostrare Dio, dovremmo farlo: l'uomo dovrebbe diventare creatore di Dio. Parimente bisogna ritenere vero tutto quello che ei è stato rivelato da Dio, ma non domandare in qual modo sia vero, che è ciò che non potremo mai comprendere. Sulla verità rivelata e sulla coscienza di Dio si appoggiano le seienze umane e vi trovano la loro norma di veritå; ma il fondamento stesso è verita di coscienza e non di scienza.

Come il Vico abbassa le scienze che Cartesio prediligeva e coltivava, la metafisica, la teologia, la fisica, cosí risolleva le forme di sapere che Cartesio aveva abbassate: la storia, l'osservazione naturalistica, la cognizione empiriea cirea l'uomo e la società, l'eloquenza e la poesia. O, per meglio dire, non ha bisogno di sollevarle per rivendicarle: dimostrato che le superbe verita della filosofia condotta con metodo geometrico si riducono anch'esse a nient'altro che probabilità e asserzioni aventi valore di sem̌plice coscienza, Ia vendetta delle altre forme del sapere è, nell'atto stesso, bella e compiuta, perché tutte si ritrovano ormai adeguate alla medesima altezza o bassczza che si dica. L'idea di una scienza umana perfetta, che respinga da sé un'altra indegna di questo nome perché fondata non sul ragionamento ma sull'autorità, è chiarita illusoria. L'autorita delle proprie e delle altrui osservazioni e credenze, l'opinione generale, la tradizione, la coscienza del genere umano, vengono restaurate nell'ufficio che hanno sempre avuto e che ebbero nello stesso Cartesio; il quale (come suole accadere) disprezzò quel che egli possedeva in gran copia e di eui si era potentemente giovato, e, uomo dottissimo, sereditò la dottrina e l'erudizione, come chi si è nutrito può darsi il lusso di parlare con disdegno del cibo 
che è già sangue nelle sue vene. La polemica di Cartesio contro l'autorità si era provata, per alcuni rispetti, benefica, avendo scosso la troppo vile servitú di star sempro sopra l'autorità. Ma che non regni altro che il proprio individuale giudizio, che si pretenda rifare da cima a fondo il sapere sulla propria individuale coscienza, che si giunga (come fece il Malebranche) ad augurare perfino di vedere bruciati tutti i filosofi e poeti antichi e di tornare alla nudità di Adamo; è una follia o, per lo meno, un eccesso, dal quale conviene rifuggire nel giusto mezzo. E il giusto mezzo è di seguire il proprio giudizio, ma con qualche riguardo all'autorità; di congiungere insieme, cattolicamente, la fede con la critica circoscritta dalla fede e giovevole alla fede stessa: in modo conforme al carattere indelebile di mera probabilità che ha il sapere o la scienza umana, in modo avverso all' indirizzo della Riforma, pel quale lo spirito interno di ciascuno si fa divina regola delle cose che si devono credere.

C'è, per altro, un gruppo delle scienze cartesiane al quale par che il Vico riconosca, come i suoi predecessori del Rinascimento, un posto privilegiato; vale a dire, non di coscienza, ma di vera e propria scienza, non nella certezza, ma nella verità: le discipline matematiche. Sono. queste, secondo lui, le sole conoscenze possedute dall'uomo in modo del tutto identico a quello del sapere divino, e cioè perfetto e dimostrativo. E non già, come Cartesio aveva creduto, per effetto del loro carattere di evidenza. L'evidenza, usata nelle cose fisiche e nelle agibili, non dà una verità della stessa forza che nelle matematiche. Né le matematiche sono per sé evidenti: con quale chiara e distinta idea si potrebbe concepire che la linea consti di punti che non hanno parti? Ma il punto impartibile, che non si può concepire nelle cose reali, si può, invece, definire; e col definire certi nomi, l'uomo si crea gli ele- 
menti delle matematiche, coi postulati li porta all'infinito, con gli assiomi stabilisce certe verità eterne, e con questi infiniti e con questa eternità disponendo i loro elementi, egli fa il vero che insegna. La forza delle matematiche nasce, dunque, non dal criterio cartesiano, ma appunto dall'altro enunciato dal Vico; non dall'evidenza, ma dalla conversione del conoscere col fare: * mathematica demonstramus, quia verum facimus ». L'uomo prende l'uno e lo moltiplica, prende il punto e lo disegna; e crea i numeri e le grandezze che egli conosce perfettamente perché opera sua. Le matematiche sono scienze operative, e non solo nei loro problemi, ma negli stessi teoremi, che volgarmente si stimano cosa di mera contemplazione. Per tal ragione esse sono anche scienze che dimostrano per cause, contrariamente all'altra opinione volgare che esclude dalle matematiche il concetto di causa; sono, anzi, le sole, tra le scienze umane, che davvero provino per cause. Da questo procedere provengono le loro verità meravigliose; e tutto l'arcano del metodo geometrico consiste nel definire prima le voci, e cioè fare i concetti coi quali si abbia a ragionare; poi stabilire alcune massime comuni, nelle quali colui col quale si ragiona convenga; finalmente, se bisogna, domandare cosa che per natura si possa concedere aftine di poter dedurre i ragionamenti, i quali senza una qualche posizione non verrebbero a capo; e con questi principî da verità piú semplici dimostrate procedere fil filo alle piú composte, e le composte non affermare se prima non si esaminino una per una le parti che le compongono.

Si direbbe che il Vico sia circa il valore delle matematiche affatto d'accordo con Cartesio, dal quale differisca soltanto nella fondazione di quel valore. E, posto che la sua fondazione debba considerarsi piú profonda, tanto piú ne verrebbe rafforzato ed esaltato l'ideale matematico, prefisso alla scienza da quello. Se l'unica conoscenza perfetta 
che lo spirito umano raggiunga è quella matematica, $\grave{e}$ chiaro che sopra essa bisogna sorreggersi e alla stregua di essa modellare o giudicare le altre. Il Vico, insomma, si sarebbe mosso per dare torto a Cartesio e gli avrebbe procurato una migliore ragione che quegli non sospettava. Ma, quantunque cosí sembri a prima vista (e cosí abbia pensato qualelie interpetre), osservando meglio si scorge che la gran perfezione che il Vico attribuisce alle matematiche è piú apparente che reale; che la sicurezza che egli vanta di quel procedere, è, per sua medesima confessione, acquistata a spese della realtà; e che, insomma, l'accento della teoria non eade tanto sulla verita di quelle discipline quanto sulla loro arbitrarietà. $\mathrm{E}$ in questo risalto dato al carattere di arbitrarietà egli differisce non solo dai ricordati filosofi del Rinascimento, ma anche da Galileo e dalla sua scuola .

L' nomo infatti (egli dice), andando attorno a investigare la natura delle cose, e accorgendosi finalmente di non poterla in niun modo conseguire, perehé non ha dentro di sé gli elementi onde sono eomposte, c, anzi, li ha tutti fuori di sé, è condotto via via a volgere a profitto questo stesso vizio della sua mente; e con l'astrazione (non, s'intende, con l'astrazione sulle cose materiali, perché il Vico non assegna origine empirica alle matematiche, ma con l'astrazione che si esereita sugli enti metafisiei) si foggia due cose, « duo sibi confingit $»$ : il punto da disegnare, e l'unita da moltiplicare. Entrambi finzioni (utrumque fictum), perché il punto disegnato non è piú punto e l'uno moltiplicato non è piú uno. Indi, da quclle finzioni, di proprio arbitrio (proprio iure) assume di procedere all'infinito, sieché le linee si possano condurre nell'immenso,

1 Si veda sulla storia della gnoseologia delle matematiche fino al Vico il mio saggio cit., pp. 258-67. 
l'uno moltiplicare per l'innumerabile. A questo modo costruisce per suo uso un mondo di forme e numeri, che egli abbraceia tutto dentro di sé; e col prolungare, col tagliare, col comporre le linee, con l'aggiungere, togliere e computare i numeri, fa infinite opere e conosce infiniti veri. Non puó definire le cose e definisec nomi; non può attingere gli elementi reali e si contenta di elementi immaginarî, dai quali sorgono idee che non ammettono alcuna controversia. Simile a Dio, «ad Dei instar », da nessun sostrato materiale, e quasi dal niente, crea punto, linea, superficie: il punto ehe è posto come quello che non ha parti; la linea come l'escurso del punto, ossia la lunghezza priva di larghezza e di profondità; la superficie, come l'incontro di due linee diverse in uno stesso punto, eioè la lunghezza e la larghezzal senza la profondita. Cosí le matematiche purgano il vizio della scienza umana, di avere sempre le cose fuori di sé e di non aver essa fatto ciò che vuole conoseere. Quelle fanno ciò che conoscono, hanno in sé medesime i loro elementi o si configurano, percio, a somiglianza perfetta della scienza divina (scientice divince similes evadunt).

A chi legge queste e altrettali descrizioni e celebrazioni vichiane del procedere matematico, par d'avvertire come un'ombra d'ironia, se non proprio intenzionale, certamente risultante dalle cose stesse. La fulgida verita delle matematiche nasce, dunque, dalla disperazione della verità; la loro formidabile potenza dalla riconosciuta impotenza! La somiglianza dell'uomo matematico con Dio non è troppo diversa da quella del contraffuttore di un'opera col suo autore: eiò che Dio è nell'universo della realtà, l'uomo è, sí, nell'universo delle grandezze e dei numeri, ma questo universo è popolato di astrazioni e finzioni. La divinita conferita all'uomo è, quasi, divinita da burla.

Per effetto della diversa genesi ehe il Vico assegna alle 
matematiche, anche la loro efficacia viene assai ristretta. Le matematiche non stanno piú, come per Cartesio, al sommo del sapere umano, scienze aristocratiche, destinate a redimere e a governare le scienze subalterne; ma occupano una cerchia, per quanto singolare, altrettanto ben circoscritta, fuori della quale se mai esse si provano a uscire, pérdono, d'un subito, ogni loro mirabile virtú.

Il potere delle matematiche incontra ostacoli a parte ante e a parte post: nel loro fondamento $\mathrm{c}$ in quel che a loro volta sono in grado di fondare. Nel loro fondamento, perché se creano i loro elementi, cioè le finzioni iniziali, non creano la stoffa in cui queste sono ritagliate, e che a esse, non meno che alle altre scienze umane, è fornita dalla metafisica, la quale, non potendo dar loro il proprio soggetto, ne dà certe immagini. Dalla metafisica la geometria toglie il punto per disegnarlo (cioè, per annullarlo come punto); e l'aritmetica l'uno per moltiplicarlo (cioè, per distruggerlo come uno). E poiché la verità metafisica, per quanto certa appaia alla coscienza, non è dimostrabile, le matematiche, in ultima analisi, riposano anch'esse sull'autorita e sul probabile. Ciò basta a svelare la fallacia di ogni trattazione matematica che si tenti dalla Metafisica. Il Vico sembra ammettere una specie di circolo tra geometria e metatisica, la prima delle quali riceverebbe il suo vero dalla seconda e, ricevutolo, to rifonderebbe nella stessa metafisica, confermando reciprocamente la scienza umana con la divina. Ma questo concetto (che è piú che contestabile e si può dichiarare senz'altro incoerente e contradittorio) richiama, in ogni caso, l'uso metafisico, o piuttosto simbolico e poetico che della matematica fecero $\mathrm{Pi}$ tagora e altri filosofi antichi e del Rinascimento, e non ha nulla da vedere con una filosofia trattata matematicamente al modo dei cartesiani. La geometria sarebbe, a giudizio del Vico, l'unica ipotesi per la quale dalla metafisica sia dato 
passare alla fisica; ma rimarrebbe in tale accezione un'ipotesi, nna probabilita, qualcosa di mezzo tra la fede e la critica, tra l'immaginazione e il ragionamento, quale rimane sempre la metafisica e, in genere, la scienza umana, secondo il modo di vedere del Vico in questa prima forma della sua gnoseologia.

Come non fondano la metafisica dalla quale anzi derivano, cosi le matematiche non sono neppure in grado di fondare le altre scienze, che pure seguono a esse nell'ordine di derivazione. Tutte le materie, diverse dai numeri e dalle misure, sono affatto incapaci di metodo geometrico. La fisica non è dimostrabile; se potessimo dimostrare le cose fisiche, le faremmo (si physica demonstrare possemus, faceremus); ma non le facciamo e percio non possiano darne dimostrazione. L'introduzione del metodo matematico nella fisica non ha giovato a questa disciplina, che fece scoperte grandi senza quel metodo, e nessuna né grande né piccola ha fatta mercé di esso. La fisica moderna somiglia, in verità, a una casa che gli antenati hanno riccamente arredata e di cui gli eredi non hanno accresciuto la suppellettile, ma si divertono solamente a eangiarla di posto e a disporla in modi nuovi. É necessario perciò restaurare e sostenere, in fisica, l'indirizzo sperimentale contro quello matematico: l'indirizzo inglese contro quello francese, il cauto uso che delle matematiche fecero Galileo e la sua scuola contro l'incauto e arrogante dei cartesiani. A ragione in Inghilterra si proibisce l'insegnamento della fisica matematica: cotal metodo non procede se non prima definiti i nomi, fermati gli assiomi e convenute le domande; $\mathrm{ma}$ in fisica si hanno a definire cose e non nomi, non vi ha convenzione che non sia contrastata, né si può domandare cosa alcuna alla ritrosa natura. Onde, nel migliorc dei casi, quel metodo si risolve in un puro e innocuo verbalismo: si espongono le osservazioni fisiche con la dicitura: «per la de- 
finizione IV , " per il postulato II , * pcr l'assioma III », c si conclude con le solenni abbreviature: «Q.e.d.»; ma non si svolge nessuna forza dimostrativa e la mente resta dipoi in tutta la libertà di opinare che possedeva innanzi di udire tali metodi strepitosi. Il Vico non sa astenersi, a tal proposito, da paragoni satirici. Il metodo geometrico (egli dice), quando è nel suo legittimo dominio, opera senza farsi sentire, e, ove fa strepito, segno è che non opera: appunto come negli assalti l'uomo timido grida e non ferisce, l'uomo d'animo fermato tace e fa colpi mortali. E ancora: il vantatore del metodo geometrico in cose in cui quel metodo non trae necessità di consentire, quando pronuncia: «questo è assioma 》 0 "questo è dimostrato», è simile al pittore che a immagini informi, le quali per sé non si possano riconoscere, scriva sotto: « questo è uomo», "questo è satiro », "questo è leone », e via discorrendo. Onde accade che col medesimo metodo geometrico Proclo dimostri i principî della fisica aristotelica, Cartesio i suoi, se non tutti opposti, certamente diversi; eppure furono due geometri, dei quali non si può dire che non sapessero usare il metodo. Quel che bisognerebbe, se mai, introdurre nella fisica sarebbe non il metodo ma la dimostrazione geometrica; ma questa è proprio ciò che non è dato introdurvi. Meno ancora è possibile nelle altre scienze via via piú corpulente e piú concrete: meno che in ogni altra, nelle seienze morali. E perciò, non potendosi usare la cosa, in cambio si abusa tanto del nome; e, come il titolo di \& signore », rifiutato un tempo da Tiberio perché troppo superbo, si dả ora a ogni vilissimo nomo, cosí quello di * dimostrazione ", applicato a ragioni probabili e talora apertamente false, ha sminuito la vencrazione che si deve alla verità.

Per le matematiche stesse il Vico scorge pericoli nella sostituzione dei metodi analitici ai geometrici o sintetici. 
E dubita che la nuova meccanica sia frutto davvero dell'analisi, la quale attutisce l'ingegno, ossia la facoltà inventiva, e, certa nel risultato (opere), è oscura nella via (opera), laddove il metodo sintetico è tum opere tum opera certissimo. L'analisi adduce le sue ragioni aspettando se per caso si diano le equazioni che cerca, e sembra un'arte d'indovinare, o una macchina piuttosto che un pensiero. Per analoghe considerazioni il Vico non teneva in alcun pregio le topiche piú o meno meccaniche e le arti lulliane e kircheriane dell' invenzione e della memoria.

La simpatia per lo sperimentalismo che, come si è visto, stacca il Vico dall'indirizzo francese e cartesiano o lo avvicina piuttosto a quello italiano e inglese, a Galileo e al Bacone, lo rende altresí nemico dell'aristotelismo e dello scolasticismo. Esortando egli a cercare i particolari e a valersi del metodo induttivo; affermando che il genere untano era stato arricchito di innumerevoli verità dalla fisica, la quale, mercé il fuoco, le macchine e gli strumenti, si era fatta operatrice di cose simili a peculiari opere della natura; raccomandando la propria metafisica come tale che serve bene (ancillantem) alla fisica sperimentale; non può nou riconoscere ben meritato il discredito in cui era caduta la fisica aristotelica, troppo (egli diceva) universale. $E$ se a Cartesio rimproverava l'introduzione delle forme fisiche nella netafisica, e con ciò la tendenza verso il materialismo, Aristotele e gli scolastici sono poi da lui accusati dell'errore opposto, cioè di aver voluto introdurre le forme metafisiche nella fisica. Come Bacone, egli stima che il sillogismo e il sorite non producano nulla di nuovo e ripetano ciò che è gia contenuto nelle premesse; e mette in chiaro i molteplici danni che gli universali aristotelici cagionano in tutte le parti del sapere: nella giurisprudenza, in cui le vuote generalità soffocano il senno legislativo; nella medicina, che bada piuttosto a tenere in piedi i si- 
stemi che a sanare gl'infermi; nclla vita pratica, nella quale gli abusatori di universali sono derisi col nome di * uomini tematici ». Dagli universali derivano le.omonimie o equivoci, cause d'ogni sorta di errori. Alla diffidenza verso gli universali, intesi qui nel senso di concetti generali o astratti, risponde nel Vico (com'era stato caso frequente presso gli antiaristotelici della Rinascenza) l'esaltazione delle idee platoniche, delle forme metafisiche, o, come egli anche le chiama, dei generi, modelli eterni degli oggetti e infiniti per perfezione. Nominalista nelle matematiche, sospettoso del nominalismo in tutti gli altri campi del sapere, il Vico asserisce la realta delle forme o delle idee, e narra come fin da giovane fosse attratto da questa dottrina, insegnatagli da un suo maestro che era scotista e perciò seguace di quella tra le filosofie scolastiche che piú si approssimava alla platonica.

Considerata nella sua interezza, la prima gnoseologia del Vico non è intellettualistica, non è sensistica e non è veramente speculativa; ma contiene tutte tre queste tendenze che si compongono in certo modo tra loro, non col sottomettersi gerarchicamente a una tra esse, ma col sottomettersi tutte alla riconosciuta incompiutezza della scienza umana. Il suo intento sarebbe di fronteggiare, con un sol movimento tatico, dommatici e scettici, contro i primi negando che si possa sapere tutto e contro i secondi che non si possa sapere cosa alcuna; ma riesce invece a un'affermazione di scetticismo o agnosticismo, nella quale non manca neppure qualche tratto mistico. Il sapere divino è sapere unitario, quello umano è la frammentazione dell'unità; Dio sa tutte le cose perché conticne in sé gli elementi dai quali le compone tutte; l'uomo si studia di eonoscerle col ridurle in pezzi. La scienza umana è una sorta di anatomia delle opere di natura, e viene dividendo l'uomo in corpo e anima, e l'anima in intelletto e volontà, e dal corpo astrae la figura e il moto, e da 
questi l'ente e l'uno; onde la metafisica contempla l'ente, l'aritmetica l'uno e la sua moltiplicazione, la geometria la figura e le sue misure, la meccanica il moto dell'àmbito, la fisica il moto del centro, la medicina il corpo, la logica la ragione, la morale la volontà. Ma accade di questa anatomia come di quella del corpo umano, circa la quale i piú acuti fisiologi dubitano se per effetto della morte e della stessa dissezione sia piú possibile indagare il vero sito, struttura e uso delle parti. L'ente, l'unità, la figara, il moto, il corpo, l'intelletto, la volontà sono altro in Dio, nel quale fanno uno, altro nell'uomo in cui restano divisi: in Dio vivono, nell'uomo periscono. La percezione chiara e distinta, nonché prova di forza, è prova di debolezza dell'intendimento umano. Le forme fisiche appaiono evidenti fintanto che non si mettono al paragone delle metafisiche: il « cogito ergo sum » è certissimo, quando l'uomo considera sé stesso, creatura finita, ma addentrandosi in Dio, che è l'unico e vero ente, egli conosce veramente non essere: con l'estensione e le sue tre misure crediamo di stabilire verita eterne, ma nel fatto \& calum ipsum petimus stultitia», perché le eterne verità sono solamente in Dio: eterno ei sembra l'assioma che il tutto è maggiore della parte, ma, risalendo ai principî, si scorge che è falso e si vede che tanta viltú di estensione è nel punto del cerchio quanto in tutta la circonferenza. Perciò (conclude il Vico) * in metafisica colui avri profittato che nella meditazione di questa scienza avrà sé stesso perduto ».

Giudicare (come pur talora è stato fatto) che in queste proposizioni il Vico sia nient'altro che un platonico o un seguace della tradizionale filosofia cristiana, e negare per conseguenza qualsiasi importanza alla sua prima gnoseologia, significherebbe attenersi a quell'erroneo modo di critica e di storia filosofica il quale, guardando alle conclusioni generali di un sistema, ne trascura il contenuto particolare, 
che solo gli dà la vera fisonomia. S'intende bene che ogni filosofo è sempre, nelle sue conclusioni finali, o agnostico o mistico o materialista o spiritualista, e via dicendo; ossia rientra in qualcuna delle perpetue categorie nelle quali si aggira il pensiero e la ricerca filosofica. Ha presentare in questo modo unilaterale i filosofi giova soltanto a favorire il pregiudizio che la storia del pensiero ripeta di continuo, sterilmente, sé medesina, passando da un errore ad un altro e abbandonando l'errore vecchio per il nuovo, che poi sarebbe anch'esso un vecchio rifatto o ritinto giovane. Il platonismo, agnosticismo o misticismo del Vico è sommamente originale perché tutto contesto di dottrine che non solo non sono inferiori al livello della filosofia contemporanea, ma lo sorpassano d'assai.

La prima di queste dottrine è la teoria del conoscere come conversione del vero col fatto, sostituita al tautologico criterio della percezione chiara e distinta. Quantunque per il Vico quella conversione rappresenti un ideale inconseguibile dall' uomo, non pertanto con essa viene esattamente determinata la condizione e la natura della conoscenza, l'identitả del pensiero e dell'essere, senza la quale il conoscere è inconcepibile. La seconda è la svelata natura delle matematiche, singolari per la loro origine tra le altre conoscenze umane, rigorose perché arbitrarie, ammirevoli ma inette a dominare e a trasformare il restante sapere umano. La terza dottrina, finalmente, è la rivendicazione del mondo dell'intuizione, dell'esperienza, della probabilita, dell'autorità, di quelle forme tutte che l'intellettualismo ignorava o negava. In questi punti l'agnostico, il platonico, il mistico Vico non era né agnostico né mistico né platonico, e compieva un triplice progresso sopra Cartesio, che, sotto tutti e tre questi aspetti, veniva da lui definitivamente criticato.

Dove, invece, Cartesio sopravanzava ancora il Vico era, 
per l'appunto, in quel dommatismo di cui il Vico non voleva a niun conto sapere. Riuscisse o no, Cartesio tentava una scienza umana perfetta, dedotta dall'interna coscienza; e il Vico, giudicando troppo superbo il filosofo francese e disperando del tentativo, asseriva invece la trascendenza della verità, si appoggiava alla rivelazione $e$ si restringeva a dare una metafisica "humana imbecillitate dignam». La sua era una gnoseologia dell'umiltà, come quella di Cartesio della superbia. Ora, il Vico non poteva progredire anche per questo verso se non ismettendo almeno una parte della sua umiltà e acquistando qualcosa della superbia di Cartesio; introducendo nel suo spirito cattolico un po' del lievito di quello spirito protestante che gli sembrava cosi pericoloso; provandosi a concepire una filosofia alquanto meno degna dell'umana debolezza e tanto piú degna dell'uomo, che è debole e forte insieme, è uomo ed è Dio. E questo progresso è manifesto nella forma successiva del suo pensiero. 
LA SECONDA FoRMa DELla gNoSEOLOGIA VICHIANA

La pleta dedizione del suo animo al cattolicismo del suo tempo e del suo paese, lo legavano saldamente alla gnoseologia e metafisica platonico-cristiana; la quale, per questi ostacoli psicologici, non poteva sviluppare nella mente di lui le contradizioni di cui era pregna. L'idea di Dio lo domava e lo sorreggeva insieme; ed egli non aveva l'andacia né sentiva il bisogno d'investigare a fondo quale valore sia da attribuire alla rivelazione, o se sia concepibile un Dio fuori del mondo, o come l'uomo possa affermare Dio senza in qualche modo dimostrarlo e percio crearlo lui. Per far si che il Vico aprisse e in parte percorresse una nuova via, la quale avrebbe condotto lo spirito umano al superamento della concezione platonico-cristiana, era indispensabile che la Provvidenza (per servirci fin da ora di un concetto vichiano, che verrà illustrato piú oltre) adoperasse verso di lui un inganno, e con Iungo e tortuoso giro lo menasse all'imboccatura della nuova via, non lasciandogli sospettare dove questa avrebbe messo capo.

Gli scritti, nei quali il Vico espose la sua prima gnoseologia, il De ratione studiorum, il De antiquissima italorum sapientia, e le polemiche relative, appartengono al 
quadriennio 1708-1712. Nel decennio che seguí, il Vico fu tratto a darsi sempre piú alle ricerche sulla storia del diritto e della civiltả. Lesse Grozio per prepararsi a scrivere la vita di Antonio Carafa, e s'ingolfò nei dibattiti sul diritto naturale; intensificò gli studî sul diritto romano e sulla scienza del diritto in genere, per rendersi degno di una cattedra di giurisprudenza nella università napoletana; ripensò alle origini delle lingue, delle religioni, degli Stati, poco soddisfatto delle tesi storiche da lui sostenute nel De antiquissima, e forse anche intimamente scosso da qualche critica che coglieva giusto, fattagli da un recensente del Giornale dei letterati; l'insegnare rettorica, che era il suo mestiere, gli porgeva continua occasione a meditare sulla natura e la storia della poesia e delle forme del linguaggio. Cosicché, se non è esatto dire che il Vico fu condotto al suo nuovo orientamento, culminante nella seconda Scienza nuova, mercé un processo non filosofico ma filologico (essendo chiaro che un orientamento filosofico non può nascere se non da un processo egualmente filosofico), è indubitabile che il materiale e lo stimolo pel suo nuovo pensiero gli furono offerti dagli studî filologici.

Attraverso i quali egli ebbe a farc un'esperienza solenne: cioè, che quella materia di studio non poteva essere e non era elaborata dal suo pensiero senza l'ajuto di certi principî necessarî, che gli si ripresentavano in ogni parte della storia da lui presa a meditare. Un tempo gli era sembrato che le scienze morali, ragguagliate al metodo matematico, occupassero, quanto a sicurezza, l'infimo posto. Ora, nella quotidiana familiarita con quelle scienze, gli si veniva scoprendo il contrario: niente di piú sicuro del fondamento delle scienze morali.

E quella loro sicurezza non era la semplice evidenza cartesiana; nella quale l'oggetto, per intrinseco che si dica, rimane estrinseco; ma era una sicurézza davero intrinseca, 
intrinsecamente ottenuta. Nel ripiegarsi con la mente sui fatti della storia, il Vico sentiva di appropriarsi meglio qualcosa che gia gli apparteneva, di rientrare in possesso di proprî beni. Egli ricostruiva la storia dell'uomo; e che cosa era la storia dell'uomo se non un prodotto dell'uomo stesso? Chi fa la storia se non la fa l'uomo, con le sue idee, i suoi sentimenti, le sue passioni, la sua volontà, la sua azione? E lo spirito umano, che fa la storia, non è quello stesso che si adopera a pensarla e a conoscerla? La verità dei principî generatori della storia nasce, dunque, non dalla forza dell'idea chiara e distinta, ma dalla connessione indissolubile del soggetto con l'oggetto della conoscenza.

Il che importava che la scoperta che il Vico ora compiva, la verita che egli ora riconosceva alle scienze morali, era la visione di un nuovo nesso del principio gnoseologico gia da lui formolato nel periodo precedente della sua speculazione, ossia del criterio della verità riposto nella conversione del vero col fatto. La ragione da lui addotta, per la quale l'uomo può avere perfetta scienza del mondo umano, è per l'appunto che il mondo umano l'ha fatto l'uomo stesso; e ove avvenga che chi fa le cose esso stesso le narri, ivi non può essere piú certa l'istoria».

Con questo riattacco alla precedente teoria l'affermazione cirea la possibilita delle scienze morali non prese, soggettivamente, nello spirito del Vico l'importanza e non portò le conseguenze di una rivoluzione, che gli sconvolgesse da cima a fondo l'assetto delle sue idee e lo costringesse a procurarne uno affatto nuovo. Quell'affermazione parve a lui, da una parte, una conferma della sua vecchia dottrina, un esempio aggiunto agli altri che aveva già recati di scienza perfetta (scienza divina dell' universo e scienza umana del mondo matematico); e dall'altra, un'estensione del campo conoscitivo, i cui limiti (perché certi limiti sussistevano sempre) aveva tracciati dapprima in modo troppo 
stretto. Prima, aveva circoscritto una breve sfera luminosa in mezzo a un vasto campo buio o fiocamente illuminato; ora, la sfera luminosa si ampliava di ún tanto, e di altrettanto scemava la zona tenebrosa. Ampliamento che non lo gettava punto in conflitto con le sue convinzioni religiose, e, anzi, sembrava favorirle ed esserne favorito. La religione non insegna forse la liberta, responsabilita ${ }^{\circ} \mathrm{e}$ consapevolezza che l'uomo ha dei proprî atti e fatti?

Il Vico non senti dunque il bisoguo di scrivere un nuovo libro metafisico, perché gli sembrò che bastasse aggiungere una postilla al gia scritto e ritoccare alquanto le sue precedenti affermazioni. La sua nuova gnoseologia, tenendo fermo il criterio generale della verità contrapposto al criterio cartesiano - e cioè, che solo chi fa le cose le conosce, - divideva le cose tutte nel mondo della natura e nel mondo umano; e osservando che il mondo della natura è stato fatto da Dio e perciò Dio solo ne ha la scienza, restringeva l'agnosticismo solamente al mondo fisico, e dichiarava, per contrario, che del mondo umano, come fatto dall'uomo, l'uomo ha la scienza. Elevava cosi le conoscenze, dapprima meramente indiziarie e probabili, circa le cose dell'uomo al grado di scienza perfetta; ed esprimeva maraviglia che $i$ filosofi si studino con tanto impegno di conseguire la scienza del mondo naturale, chiuso all'uomo, e trascurino il mondo umano o civile o delle nazioni (come anche lo chiamava), del quale è possibile conseguire scienza. Di questo erramento trovava la cagione nella facilita che la mente umana, immersa e seppellita nel corpo, prova a sentire le cose del corpo, e nello sforzo e fatica che le costa d'intendere sé medesima: come l'occhio corporale vede tutti gli oggetti fuori di sé e, per vedere sé stesso, ha bisogno dello specchio.

In ogni altra parte, le sue idee restavano immutate. Di là dal mondo umano, il mondo soprannaturale, inaccessi- 
bile all'uomo, e il mondo naturale, che era in certo senso anch'esso soprannaturale; di là dalla scienza perfetta che l'uomo può avere di sé stesso, la metafisica platonico-cristiana, adatta alla debolezza, che continuava pur sempre ad affliggere l'uomo. Le discipline naturali venivano considerate sempre come semiscienze; le matematiche come una formazione astratta, validissima nell'astratto, priva di forza innanzi al reale. Il sillogismo di Aristotele, il sorite degli stoici, il metodo geometrico dëi cartesiani erano perseguitati dallo stesso odio di prima, e con lo stesso amore celebrata l'induzione che il Verulamio, " gran filosofo insieme e politico , commendava e illustrava nel suo $\mathrm{Or}$ gano, e che gl'inglesi adoperavano con gran frutto della sperimentale filosofia.

Un ravvedimento circa l'applicabilità del metodo geometrico potrebbe sembrare la frequente asserzione del Vico che la scienza delle cose umane sia da lui costruita con uno « stretto metodo geometrico ». Ma, anche a lasciar andare che la struttura della Scienza nuova è proprio l'opposto di quella geometrica, è un fatto che, nel tempo stesso e negli stessi libri, egli non cessa di mettere in guardia contro l'uso del metodo matematico nelle cose fisiche e morali, il quale * ove non sono figure di linee o di numeri o non porta necessità, spesso invece di dimostrare il vero può dare apparenza di dimostrazione al falso »; onde il preteso ravvedimento sarebbe una palmare contradizione, se non gli si potesse dare un significato che ristabilisce interamente la coerenza nelle idee del Vico. Un significato assai semplice, perché, riconosciuta ormai alle scienze morali non meno che alla geometria la potenza di convertire il vero col fatto, esse potevano e dovevano svolgersi con metodo analogo a quello sintetico della geometria, con cui da vero si passa a immediato vero, e seguire il mondo umano dai suoi inizî ideali nei suoi progressi fino 
alla sua perfezione, sicché lo studioso non doveva sperare di poter intendere le loro dottrine per salti, ma doveva percorrerle per gradi da capo a piedi, senza recalcitrare alle conclusioni inaspettate che ne uscissero, come non si recalcitra a quelle della geometria, e attendendo soltanto a esaminare la saldezza del nesso tra premesse e conseguenze. Era, dunque, codesto un metodo chiamato geometrico per analogia o per sineddoche, ma in effetti intrinsecamente speculativo, da non confondere con l'applicazione della matematica alle cose morali, quale ne avevano dati esempî i cartesiani e lo Spinoza.

Né si può concedere senza liserve il giudizio di alcuni interpetri: che il Vico in realtà, con l'ammettere una scienza dell' romo da investigarsi nelle modificazioni stesse della mente umana, si ravicinasse e facesse seguace di Cartesio; al qual nopo si suole addurre anche l'altra dichiarazione di lui, che, per pensare la sua Scienza nuova, convenisse « ridursi a uno stato di somma ignoranza, come né filosofi né filologi né libro alcuno fossero mai stati al mondo ». Certamente, il Vico con la nuova forma della sua gnoscologia entra anche lui nel soggettivismo della filosofia moderna inaugurato da Cartesio (anzi, vi era già entrato, in certo modo, con la sua dottrina attivistica della verità come rifacimento del fatto); e, in questo significato del tutto generico può dirsi, anche lui, cartesiano. Pure, se a Cartesio rimane ancora inferiore, perché il suo soggettivismo è principio non della scienza tutta ma di quella sola del mondo umano, per un altro verso si pone di sopra al filosofo francese, in quanto, per lui, la verita meditata nel mondo umano non è statica ma dinamica, non è trovata ma prodotta, è scicnza e non coscienza. Per quel che concerne poi l'esortazione a far conto come se non vi fossero mai stati libri al mondo né placiti di filosofi e di filologi, essa non importa altro se non cle 
bisogni spogliarsi di ogni pregiudizio, di ogni comune invecchiata anticipazione, di ogni corpulenza proveniente da fantasia o da memoria, per ridursi \& in istato di puro intendimento, informe di ogni forma particolare », com'e indispensabile per la scoperta e l'apprendimento di ogni nuova verití; e tanto poco qui l'esortazione ha il significato cartesiano e malebranchiano di un rifuto della crudizione e dell'autorita, che, per non dir altro, nel medesimo luogo al quale di sopra si è alluso, si trova avvertito che la Scienza nuova « suppone una grande e varia cosí dottrina come erudizione ", dalle quali prende le verita come già conosciute per valersene da termini per fare le sue proposizioni.

Nella nuova sua gnoseologia il Vico, insomma, diventa non gia piú cartesiano ma sempre più vichiano, sempre piú lui. Cartesio non pare gli servisse neppure come tramite attraverso cui giungere alla persuasione della possibilita di costruire con la mente la scienza della mente. Il tramite vero $f u$ il criterio stesso vichiano della verità, messo a contatto con le osservazioni che l'autore venne facendo nel corso dei suoi studî storici. Che se si volessero cercare precedenti, nella storia della filosofia, alla seconda forma della gnoseologia del Vico, bisognerebbe, circa la divisione dei due mondi di realta e delle due sfere di conoscenza, c circa la preferenza manifestata per le indagini morali rispetto alle naturali, correre col peusiero alla posizione assunta da Socrate verso i fisiologi del suo tempo, al sentimento di religioso mistero onde il filosofo attico arretrava innanzi al mondo della natura e si rivolgeva a indagare la conformazione dell'animo umano. $\mathrm{E}$, circa la maggiore trasparenza delle scienze morali in quanto concernono cose che l'uomo stesso ha prodotto, si potrebbe richiamare la partizione aristotelica delle scienze in fisiche, che considerano il movimento estrinseco all'uomo, 
e in pratiche e poietiche, che considerano le cose prodotte dall'uomo. La distinzione era passata nella filosofia delle scuole; e Tommaso d'Aquino parla della natura come " ordo quem ratio considerat sed non facit ", e del mondo dell'attività umana come «ordo quem ratio considerando facit ». Ma queste riferenze non sono indicate dal Vico, il quale pure assai si compiaceva nel fare omaggio dei proprî pensieri agli antichi filosofi e, ammesso anche che avessero qualche efficacia sopra di lui, è certo che tra esse e la dottrina vichiana sulla conoscibilità del mondo umano corre distanza non minore che tra la proposizione dell'onniscienza di Dio creatore e il principio gnoseologico che egli seppe ricavarne.

Di questo principio, la dottrina vichiana sulle scienze morali è né piú né meno che la prima legittima applicazione; e inesattamente il suo autore (come di solito, poi, gl'interpetri) ebbe a presentarla quale semplice estensione delle applicazioni gia date, un secondo caso aggiunto a quello gia contemplato delle scienze matematiche.

Nel caso delle scienze matematiche, il principio della conversione del vero col fatto veniva applicato solo in apparenza. Originale e vero, quel principio; originale e vera la teoria delle matematiche; del tutto artificiale e falsa la connessione delle due verità. Mancava (se non c'inganniamo) un effettivo rapporto tra il concetto di Dia che crea il mondo, e, perché lo crea, lo conosce; e quello di colui che costruisce arbitrariamente un mondo di astrazioni e, nel fare ciò, non conosce nulla o conosce soltanto (quando non è piú geometra o aritmetico ma filosofo, quando scrive non gli Elementi di Euclide ma le pagine di gnoseologia del De antiquissima) che egli procede arbitrariamente. Se le discipline matematiche foggiano i concetti a libito, se producono finzioni e non verità, esse, a dil' vero, non sono scienze né conoscenze di sorta, e non 
c'è possibilita di porle a riscontro con la scienza divina, che è scienza della reale realtả. Nelle matematiche (diceva il Vico) * l'uomo, contenendo dentro di sé un immagi$\mathrm{n}$ at o mondo di linee e di numeri, opera talmente in quello con l'astrazione, come Dio nell'universo con la realtà». Il riscontro può riuscire brillante, ma risplende, forse, di luee piuttosto metaforica che logica.

Nelle scienze morali, invece, il riscontro è tanto logico, che deve dirsi senz'altro coincidenza. Il sapere umano è, qualitativamente, il medesimo del divino, e al pari del pensiero divino conosee il mondo umano; sebbene, quantitativamente piú ristretto, non si estenda, come quello, al mondo della natura. Nel campo umano, non piú espedienti di debolezza, non piú finzioni, non piú falsificazioni : qui si è nella maggiore concretezza del conoscere. L'uómo crea il mondo umano, lo erea trasformandosi nelle cose civili; e, col pensarlo, ricrea la sua creazione, ripercorre vie già percorse, la rifà idealmente e perció conosce con vera e piena scienza. Questo è davvero un mondo, e l'uomo è per davvero il Dio di questo mondo.

Ci sembra, dunque, incontrastabile cle solamente l'applicazione del verum-factum, quale si effettua nella Scienza nuova, risponda al eriterio stabilito; e che l'altra che ne era stata anteriormente tentata per le matematiche, importante per altri rispetti e validissima a liberare gli spiriti dal pregiudizio matematico, non si possa considerare vera e propria applicazione. E, forse, il Vico ebbe talvolta qualche sentore della differenza tra le due applicazioni, la propria e la metaforica, che per solito confuse come identiche. La scienza del mondo umano (egli dice) « procede appunto come la geometria che, mentre sopra i suoi elementi il costruisce o 'l contempla, essa stessa si faccia il mondo delle grandezze; ma con tanto piú di realita quanta piú ne lianno gli ordini d'intorno alle facende 
degli uomini, che non ne hanno punti, linee, superficie o figure ». E un altro indizio della coscienza che si accendeva a tratti in lui di avere per la prima volta, nella dottrina circa il mondo umano, ritrovata una conoscenza vera e propria (non una mera finzione di conoscenza), potrebbe vedersi nell'uso assai piú convinto, piú caldo ed entusiastico che egli fa, in questo caso, dell'epiteto « divino $\gg$; ben diverso da quello freddo, se non propriamente ironico, dell' « ad Dei instar " nel De antiquissima. Le prove della Scienza nuova (dice piú d'una volta, con rapimento) « sono d' una spezie divina, e debbono, o leggitore, arrecarti un divin piacere, perocché in Dio il conoscere e il fare è una medesima cosa! ».

La conversione del vero col fatto nelle scienze morali non poteva non ripercuotersi nella trattazione del certo ossia (secondo uno dei parecchi significati, e forse il principale, che il Vico attribuisce a questa parola) delle cognizioni storiche (del peculiare, certum, contrapposto al commune o verum); il che forma l'altro tratto importante della seconda gnoseologia vichiana. Nella prima gnoseologia, quelle cognizioni erano legittimate e protette, come si è visto, col parificarle a ogni altra sorta di conoscenze tutte egualmente deboli o egualmente forti, perché tutte fondate sulla probabilità e sull'autorità, sia dell' individuo (autopsia) sia del genere umano. Ma, redenta dall'autorità c dalla probabilità la conoscenza dello spirito umano e delle sue leggi, le cognizioni storiche, quantunque di loro natura fondate sempre in qualche modo sull'autorità, venivano rischiarate di nuova luce. Il certo doveva entrare in un nuovo rapporto, perché aveva ormai di fronte non un altro certo, ossia una semplice conoscenza probabile circa lo spirito umano, ma un vero, una conoscenza filosofica.

Questo rapporto è chiamato altresi dal Vico il rapporto di filosofia e filologia, la prima delle quali versa circa 
* necessaric naturce $\gg$ e contempla la ragione onde viene la scienza del vero, la seconda cirea « placita humani arbitrii » e osserva l'autorità onde viene la coscienza del certo. L' una considera l'universale, l'altra l'individuale, l'una (avrebbe detto il Leibniz) le «vérités de raison », l'altra le «vérités de fait ». Distinzione ehe non è mantenuta dappertutto, presso il Vico, nella medesima nettezza; tanto che a volte l'autorità contrapposta alla ragione diventa, secondo lui, parte della ragione stessa, o si confonde con la conoscenza dell'arbitrio umano, contrapposta a quella della volonta razionale; ma di cui è per altro chiarissimo il senso generale. E per « filologia » il Vico non intende solamente lo studio delle parole e della loro storia, ma, poiché alle parole sono annesse le idee delle cose, anzitutto la storia delle cose; onde i filologi debbono trattare di guerre, paci, alleanze, viaggi, commerci, di costumi, leggi e monete, di geografia e di cronologia, e di ogni altra cosa che si attenga alla vita dell'uomo nel mondo. La filologia insomma (nel significato vichiano, che è poi il significato esatto) abbraccia non solamente la storia delle lingue o delle letterature, ma quella altresi delle idee e dei fatti, della filosofia e della politica.

Certamente, la filologia, le verità di fatto, il certo non sempre erano stati brutalmente maltrattati come dai cartesiani. Il Grozio aveva dato esempio di vastissima erudizione storica, messa a servigio delle sue dottrine sul diritto naturale. Il Gravina, contemporaneo e connazionale del Vico, richiedeva come necessarie al giurisperito non solo la « ratiocinandi ars », ma la « latince lingua peritia» $\mathrm{e}$ la « notitia temporum $»$. E il Leibniz, or ora ricordato, riasseriva l'importanza dell'erudizione contro i cartesiani e padroneggiava da gran signore i piú svariati aneddoti storiei, che profondeva a piene mani nei suoi libri. Ma il Vico notava che filosofia e filologia rimanevano tuttavia, ai suoi 
tempi, estranee l'una all'altra, come erano state quasi del tutto presso i greci e i romani: i tanti luoghi di storici, oratori, filosofi e poeti, che il Grozio accumulava, costituivano un puro ornamento; e il medesimo il Vico avrebbe giudicato forse (se ne avesse avuto conoscenza ce ne avesse comunicato il suo giudizio) del largo uso che il Leibniz faceva della storia. Leggendo i libri dei filologi, egli provava un tal senso di vuoto e di fastidio per l'affastellamento inintelligente delle notizie storiche, che era tratto quasi a dare ragione (e dové darla per qualche tempo incondizionatamente) a Cartesio e al Malebranche nel loro odio contro l'erudizione. Senonché (pensò dipoi), quei due filosofi, in cambio di sprezzare l'erudizione, avrebbero dovuto piuttosto indagare se non fosse stato possibile richiamare li filologia ai principî della filosofia; e i filologi, da parte loro, invece di arrecare $i$ fatti a pompa di erudizione, debbono industriarsi di elaborarli a fini di scienza. La filologia è da ridurre a scienza: ecco il pensiero del Vico circa i rapporti del certo col vero, della filologia con la filosofia.

Che cosa vuol dire ridurre la filologia, o la storia, che è lo stesso, a scienza o a filosofia? A rigore, la riduzione non è possibile, non perché si tratti di cose eterogenee, ma anzi perché quelle sono omogenee: la storia è già intrinsecamente filosofia; non è possibile proferire la piú piccola proposizione storica senza plasmarla col pensiero, cioè, con la filosofia. Ma poiché questo presupposto filosofico della filologia allora non era avvertito (come non fu molto spesso neppure nei tempi seguenti), e facilmente veniva negato; poiché i piú, come sappiamo, o concepivano un'aristocratica filosofia geometrica, disdegnosa e aborrente dal profanum vulgus dei casi storici, ovvero (come faceva prima il Vico stesso) una filosofia e una storia egualmente poco rigorose e meramente opinabili; il Vico, mutato il suo punto di vista filosofico, raggiunta la coscienza 
del metodo speculativo nella scienza dell'uomo, inteso piú profondamente lo spirito umano, doveva scorgere quanto ci fosse da riformare nella storiografia corrente, sentire il bisogno di una piú perfetta filologia come conseguenza della sua piú perfetta filosofia, e in termini gnoseologici esprimerlo con quella formola del richiamare alla filosofia la filologia, a ut hac posterior, ut par est, prioris sit consequentia ». Doveva, in altre parole, togliere la storia dalla sua condizione d'inferiorita, dalla servitú al capriccio, alla vanità, al moralismo, alla precettistica o ad altri fini estrinseci, e riconoscerle il fine proprio e intrinseco di necessario complemento del vero universale. In pari tempo, la filosofia si sarebbe riempita di storia, affiatata con la storia; e da questo affiatamento avrebbe acquistato maggiore larghezza e un senso piú vivo della realtà concreta da spiegare. Tale, senza dubbio, è uno dei significati che ha la formola vichiana del congiungimento di filosofia e filologia e della riduzione della filologia a scienza.

Ma non meno è fuori dubbio che, nel pronunziare quella formola, il Vico voleva qualcosa di piú e, di solito, intendeva qualcosa d'altro. Questo qualcos'altro può, nel modo piú diretto, essere chiarito dall'appello che egli fa al Bacone e al suo «metodo di filosofare piú accertato »: metodo espresso nel titolo del libro baconiano: Cogitata et visa, e che il Vico si proponeva di « trasportare dalle naturali alle umane cose civili». Esigeva, insomma, la costruzione di una storia tipica delle società umane (cogitare), da riscontrare poi nei fatti (videre), accertando coi fatti la costruzione ideale e avverando con la costruzione ideale i fatti, confermando la ragione con l'autorita e l'autorità con la ragione; di una scienza che fosse insieme filosofia dell'umanità e storia universale delle nazioni. Ora questa costruzione che egli esigeva, questo qualcosa di mezzo tra il cogitare e il videre, tra il pensiero e l'esperienza, questo 
misto dei due processi, è intrinsecamente diverso dalla unitì di filosofia e filologia in quanto interpetrazione filosofica dei dati di fatto. Questa interpetrazione è la storia vivente; l'altra non è né filosofia né storia, ma una scienza empirica dell'uomo e delle societa, materiata di schemi che non sono le extratemporali categorie filosofiche e neppure gl'individuali fatti storici (benché senza categorie filosofiche e senza fatti storici non potrebbero mai costruirsi): una scienza empirica, e perciò né esatta né vera, ma solamente approssimativa e probabile, e soggetta a verificazione e rettificazione da parte cosí della filosofia come della storia.

Sarebbe impossibile determinare quale di codesti due significati della filologia ridotta a storia sia quello proprio del Vico, perché nel suo pensiero si trovano tutti e due; o quale prevalga, perché effettivamente prevale ora l'uno ora l'altro, quantunque il secondo, quello empirico, sia piú di frequente formolato. Anzi si potrebbe dire che, quando Vico intitolara Scienza nuova la sua opera, il principale dei significati che dava a questo titolo * invidioso », si riferiva appunto a quella scienza empirica: alla scienza cioè che fosse insieme filosofia e storia dell' umanita, alla storia ideale delle leggi eterne sopra le quali corrono i fatti di tutte le nazioni nei loro sorgimenti, progressi, stati, decadenze e fini. Il Vico, ill realtà, non unificò mai (e non poteva) i due diversi significati, e ne serbò la duplicita, la quale, appunto perché non era distinta chiaramente, prendeva apparenza d'identita. Di qui la parziale giustiticazione di entrambe le tendenze che si sono manifestate tra gl'interpetri, dei quali alcuni vogliono che il Vico professasse e adoperasse il metodo speculativo, altri che il suo metodo fosse, nell'idea e nell'attuazione, empirico, induttivo e psicologico; gli uni che egli mirasse a dare un sistema di filosofia dell' umanità, gli altri che si propo- 
nesse una sociologia o una demopsicologia. Unilaterali entrambi, ma i secondi piú dei primi, perché se in veriti nel Vico c'è del Bacone e c'è del Platone, dell'empirista e del filosofo, quando poi si colga il carattere del suo ingegno, quando si penetri nell'intimo del suo spirito, c si partecipi ai suoi dissidî e al suo magnanimo sforzo, si deve riconoscere che il Vico, checché volesse e credesse, era della stoffa di un Platone e non di un Bacone; - che il Bacone stesso del quale egli parla è mezzo immaginato da lui, è un Bacone alquanto platonizzato; - e che la Scienza nuova gli pareva, in fondo, cosi nuova non perché fosse un'empirica costruzione baconiana (nel quale caso niente di piú vecchio, bastando ricordare la Politica di Aristotele e i Discorsi del Machiavelli), ma perché era tutta pregna di una nuova filosofia, la quale, infatti, irrompe da ogni parte, attraverso tutta la sua empiria. 


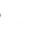


LA STRUTtura INTERNA DELla SCIENZA NUOVA

I.

a poca chiarezza circa il rapporto di filosofia e filologia, l'indistinzione dei due modi affatto diversi di concepire la riduzione della filologia a scienza, sono conseguenza e cagione insieme dell'oscurità che regna nella * Scienza nuova . Col quale nome intendiamo tutto quel complesso di ricerche e dottrine che il Vico venne mettendo fuori dal 1720 al 1730 , anzi al 1744 , c che, elaborato precipuamente nelle tre opere del De uno universi iuris principio et fine uno e della prima e seconda Scienza nuova, ha nella redazione definitiva di quest'nltima la sua forma piú sviluppata e matura, alla quale principalmente giova riferirsi.

La Scienza nuova, in modo conforme al vario significato dei termini e del rapporto tra filosofia e filologia, consta di tre ordini di ricerche: filosofiche, storiche ed empiriche; e contiene tutt'insieme una filosofia dello spirito, una storia (o gruppo di storie), e una scienza sociale. Alla prima appartengono le idee, enunciate in alcuni assiomi o dignita e sparse altresí nel corso dell'opera, sulla fantasia, sull' universale fantastico, sull'intelletto e l'universale logico, sul mito, sulla religione, sul giudizio morale, sulla forza e il diritto, sul certo e il vero, sulle 
passioni, sulla provvidenza, e tutte le altre determinazioni concernenti il corso o sviluppo necessario della mente ossia dello spirito umano. Alla seconda, ossia alla storia, l'abbozzo di una storia universale delle razze primitive dopo il diluvio e dell'origine delle varie civiltả; la caratteristica della societa barbarica o eroica antica in Grecia e specialmente in Roma sotto l'aspetto della religione, del costume, del diritto, del linguaggio, della costituzione politica; l'indagine sulla poesia primitiva, che si esemplitica poi piú lalrgamente con la determinazione della genesi e del carattere dei poemi omerici; la storia delle lotte sociali tria patriziato e plebe e dell'origine della democrazia, studiata anch'essa principalmente in Roma; la caratteristica della barbarie ricorsa, ossia del medioevo, anch'esso studiato in tutti gli aspetti della vita e raffrontato con le societả barbariche primitive. Finalmente, alla scienza empirica si richiama il tentativo di stabilire un corso uniforme nelle nazioni, concernente la successione cosi delle forme politiche come delle altre e correlative manifestazioni teoretiche e pratiche della vita, e i tanti tipi che il Vico viene delineando del patriziato, della plebe, del feudalesimo, della patria potestà e della famiglia, del diritto simbolico, del linguaggio metaforico, della scrittura geroglitica, e via discorrendo.

Ora se questi tre ordini di ricerche e dottrine fossero stati logicamente distinti nella mente del Vico e solo letterariamente mescolati e compressi in un medesimo libro, questo sarebbe potuto riuscire disordinato, sproporzionato, disarmonico, e perciò faticoso a chi si faccia a leggerlo, ma non veramente oscuro. Né, del resto, in linea di fatto, può dirsi che la Scienza nuova, almeno la seconda ossia l'esposizione definitiva che il Vico offri del suo pensiero, difetti di un disegno generale, abbastanza ben concepito. L'opera è divisa in cinque libri, il primo dei quali dovrebbe raccogliere i principî generali, cioè la filosofia; il secon- 
do, oltre un breve cenno sulla storia universale antichissima, descrivere la vita delle societa barbariche, e ad esso formare appendice il terzo sulla discoverta del vero Omero, e cioè sul piú cospicuo esempio della poesia barbarica; il quarto, delineare la scienza empirica del corso che fanno le nazioni; e il quinto esemplificare íl ricorso col caso particolare del medioevo. E tuttavia, a dispetto di questa bella architettura, la seconda Scienza nuova, com'è la piú ricca e compiuta, cosi è la piú oscura tra le opere del Vico. Se, d'altra parte, il Vico, pur avendo ben chiare in mente le sue idee, adoperasse una terminologia insueta o una forma troppo concisa di esposizione e troppo piena di allusioni e d'inespressi presupposti, sarebbe senza dubbio uno scrittore difficile, ma, neppure in questa ipotesi, oscuro. La quale ipotesi neanche risponde alla realtà, giacché il Vico è assai parco di termini scolastici e predilige le espressioni vive e popolari; i scrittore robusto ma non laconico, e spesso si compiace di ripetere le sue idce fermandovisi sopra a piú riprese e con molta insistenza; e mette in tavola tutte le sue carte, cioè tutto il materiale eludito dal quale gli sono state suggerite le dottrine. Né, infine, si è detto molto quando si è detto che al Vico mancava piena coscienza delle sue scoperte; perché questa coscienza manca piú o meno in tutti i pensatori e in nessuno puo essere mai piena. L'oscurità, la vera oscuritá, quella che si avverte nel Vico, e che a volte avvertiva egli stesso senza riuscir mai a trovarne la causa, non è superficiale e non nasce da cagioni estrinseche o accidentali, ma consiste veramente in oscurita d'idee, nella deficiente intelligenza di certi nessi e nella sostituzione con nessi fallaci, nell'elemento arbitrario che perciò s'introduce nel pensiero, o, per dirla nel modo piú semplice, in veri e proprî errori. Si potrebbe riscrivere la Scienza nuova rifacendone l'ordine e mutandone o schiarendone la terminologia (chi scrive ha 
fatto per suo conto questa prova), e l'oscurità persisterebbe, anzi si accrescerebbe, perché in siffatta traduzione l'opera, perdendo la forma originale, perderebbe altresí quella torbida ma possente efficacia che può tenere luogo talvolta della cbiarezza e che, dove non illumina, scuote lo spirito del lettore e propaga l'onda del pensiero quasi per vibrazioni simpatetiche.

Che cagione dell'oscurità, ossia dell'errore o degli errori del Vico, sia l'indistinzione o confusione già notata nella sua gnoseologia circa il rapporto tra filosofia, storia e scienza empirica, e sussistente non meno nel suo effettivo pensiero intorno ai problemi dello'spirito e della storia umana, risulta dall'osservare come filosofia, storia e scienza empirica si convertano a volta a volta presso di lui l'una nell'altra e, danneggiandosi a vicenda, producano quelle perplessità, equiroci, esagerazioni e temerità, che sogliono turbare il lettore della Scienza nuova. La filosofia dello spirito si atteggia ora come scienza empirica ora come storia; la scienza empirica ora come filosofia ora come storia; e la proposizione storica acquista l'universalita del principio filosofico o la generalita dello schema empirico. Per esempio, la filosofia dell' umanità assume di determinare le forme, categorie o momenti ideali dello spirito nella loro successione necessaria, c bene merita per tal rispetto il titolo o la definizione di storia ideale eterna sulla quale corrono nel tempo le storie particolari, non potendosi concepire nessun frammento, per piccolo che sia, di storia reale, dove non operi quella storia ideale. Ma poiché storia ideale è anche pel Vico la determinazione empirica dell'ordine in cui si succedono le forme delle civiltà, degli stati, dei linguaggi, degli stili, delle poesie, accade che egli concepisca la serie empirica come identica alla serie ideale e fornita delle virtú di questa; onde la pronunzia tale che debba sempre esattamente riscontrarsi nei fatti, "fosse anco che 
nell'eternita nascessero di tempo in tempo mondi infiniti $x$; il che è apertamente falso, non essendovi alcuna ragione che si ripetano in perpetuo (col « dovette, deve e dovrà) le empiriche aristocrazie di Grecia o di Roma, e le civiltà sorgano o decadano per l'appunto come sorsero o decaddero quelle antiche. E nel medesimo atto di questo assolutizzamento del corso empirico, il corso ideale si vela di un'ombra empirica, perché, reso identico all'altro, riceve il carattere empirico dell'altro, e si temporalizza, da eterno ed extratemporale che era nella concezione iniziale. Si dica il medesimo delle singole forme dello spirito, le quali, come ideali ed extratemporali, sono tutte e scmpre in ogni singolo fatto; ma il Vico, confondendole coi fatti reali e concreti che la scienza empirica fissa nei suoi schemi, viene, subito dopo averle proposte, ad abbuiarle nella loro ideale forma e distinzione. $\dot{E}$ vero che il momento della forza non è quello della giustizia; ma il tipo empirico della socicta barbarica fonảata sulla forza, appunto perché è una determinazione rappresentativa e approssimativa, e si riferisce a uno stato di cose concreto e totale, non contiene solamente forza, si anche giustizia; c quando quel momento ideale o quel tipo sono scambiati fra loro e presi come identici, da una parte il concetto filosofico della forza s'intorbida di quello di giustizia e, facendosi ibrido e contradittorio o incoerente, si sforma, dall'altra il tipo empirico della societa barbarica viene esagerato e di troppo irrigidito. La confusione dell'clemento filosofico e dell'empirico si può dire manifesta nella « dignità cle definisce la natura delle cose: « Natura di cose altro non è che nascimento di esse in certi tempi c con certe guise, le quali sempre che sono tali, indi tali e non altre nascono le cose »; dove appaiono messi insieme le guise e i tempi, la genesi ideale e la genesi empirica. Similmente, è verissimo che la storia debba procedere d'accordo con la filo- 
sofia, e che quello che è filosoficamente ripugnante non possa essere giammai storicamente accaduto; ma, poiché per il Vico la filosofia è indistinta dalla scienza empirica, egli, dove il documento gli manea e perciò nessuna filosofia è applicabile, si sente tuttavia sicuro della verita, c, riempiendo il vuoto con la congettura che gli fornisce lo schema della scienza empirica, s'illude di aver fatto ricorso a «prove metafisiche». O anche, trovandosi innanzi a fatti dubbî, anziché attendere che la scoperta di altri documenti dissipi le dubbiezze, risolve il dubbio col prenderli, come egli dice, " in conformità delle leggi », cioè sempre dello schema empirico; il che, in via d'ipotesi, è certamente lecito. Ma quell'ipotesi è, invece, pel Vico, una "verità meditata in idea ", sicché il riscontro coi fatti, che egli pure raccomanda per conferma, dovrebbe essere superfluo; o, se i fatti nel riscontro risultassero contrarî, il torto dovrebbe essere dei fatti, cioè dell'apparenza, non mai dell'ipotesi, affermata eome verità indubbia perché filosofica. Di qui la tendenza, che è nel Vico, a fare, come si dice, violenza ai fatti.

Bastino questi esempî a indicare il vizio intimo di struttura che è nella Scienza nuova, e a porre uno dei capisaldi della nostra esposizione e della nostra critica del pensiero vichiano, nel corso delle quali molti altri esempî ci si faranno spontaneamente innanzi e anche i gia dati saranno meglio schiariti. Ma un altro caposaldo che bisogna bene stabilire è che quel vizio è il vizio di un organismo sommamente robusto, e che gli ordini di ricerche che vengono dal Vico confusi sono costituiti da effettive ricerche di straordinaria novità, verità e importanza. E, insomma, il vizio medesimo ehe s'incontra di frequente presso gl'ingegni assai originali e inventivi, i quali di rado portano a perfezione nei particolari le loro scoperte; laddove gl'ingegni meno inventivi sogliono essere piú esatti e conse- 
guenti. Profondità e acume non sempre vanno insieme e con pari vigore; e il Vico, quantunque non fosse molto acuto, era sempre molto profondo.

Luce e tenebre, verita ed errore che si alternano e incrociano quasi a ogni punto della Scienza nuova, sono diversamente appresi secondo le diverse anime dei lettori e critici; anzi, in casi eminenti com'è questo del Vico, si possono scorgere in modo piú netto tali diversità. Vi sono anime restie e diffidenti, pronte a notare ogni piú piccola contradizione, inesorabili nell'esigere le prove di ogni affermazione, vigorose nel maneggiare le tenaglie dei dilemmi che stritolano senza pietà un povero grand'uomo. Per costoro l'opera del Vico (e molte altre della stessa qualità) è un libro chiuso; e, tutt'al piú, offrirà loro l'argomento per una di quelle cosí dette « demolizioni », che essi compiono con grande facilità e gusto, sebbene con searso successo, perché l'uomo da essi ucciso, dopo morto, suole restale piú vivo di prima. Ma vi sono altré anime, che alla prima parola che vada diritta al loro cuore, al primo raggio di verita che lampeggi ai loro occhi, si aprono tutte con desiderio, si abbandonano con fiducia, s'illebriano d'entusiasmo, non rogliono sapere di difetti, non scorgono difficơlta, o le difficoltà appianano subito e i difetti giustificano nel modo piú semplice, e, quando per caso scrivono, le loro seritture si configurano come a apologie ». E per costoro è da temere che la Scienza nuova sia un libro troppo aperto. Certamente, se fra questi due atteggiamenti opposti non ce ne fosse un telzo, se bisognasse risolversi di necessità per l'uno o per l'altro, sarebbe da preferire il peccato del troppo vivo amore a quello della gelida indifferenza, la troppa fede, che pur lascia eoglierc qualche aspetto del vero, alla nessuna fede che non ne laseia vedere alcuno. Ma un terzo atteggiamento è possibile, ed è doveroso pel critico: quello di non perdere mai 
di vista la luce, ma di non dimenticare le oscurità di giungere allo spirito passando oltre la lettera, ma di non trascurare la lettera, anzi di ritornarvi di continuo, procurando di mantenersi interpetre libero ma non fantasioso, amante fervido ma non cieco.

I due capisaldi stabiliti, il vizio e la virtú che si sono riconosciuti proprî della mente del Vico, la sua geniale confusione o la sua genialità confusionaria, impongono perciò come generale canone ermeneutico di andare separando per via d'analisi la schietta filosofia che è in lui dall'empiria e dalla storia con le quali è commista e quasi incorporata (e altresí queste da quella), e di notare via via gli effetti e le cause della commistione. Le scorie non possono essere considerate inesistenti, congiunte come sono all'oro nello stato di natura, ma non debbono impedire di riconoscere e purificare l'oro; o, fuori di metafora, la storia dev'esserc storia senza dubbio, ma tale non è se non è intelligente. 
LA FORMA FANTASTICA DEL CONOSCERE

(La poesia e il lingeaggio)

Delle forme dello spirito il Vico studiò, nella Scienza nuova, principalmente, e si potrebbe dire esclusivamente, quelle inferiori o individualizzanti, che egli designava tutt'insieme col nome di « certo »: - nello spirito teoretico la fantasia, nello spirito pratico la forza o arbitrio, e nella scienza empirica corrispondente alla filosofia dello spirito, la civiltà barbarica o sapienza poetica, la cui investigazione costituisce (come egli stesso dice) \& quasi tutto il corpo dell'opera .

Pcrché e come egli prendesse cosi forte interesse a codeste forme inferiori e alle societa primitive e storie barbariche che le rappresentavano, è anche qui, nell'aspetto estrinseco, spiegato dagli studî che il Vico ebbe a condurre sul diritto romano e sui tropi e le figure rettoriche, dalla tradizione umanistica ancora viva in Italia, dal culto allora rinvigorito per le scienze archeologiche, dalla curiosità che spingeva a indagare l'antichissima civiltà italiana, e via enumerando. Ma altri non pochi, nel suo tempo e nel suo stesso paese, trattarono le medesime materie senza punto acquistare la predilezione e la penetrazione del fantastico, dell'ingenuo, del violento: cose 
delle quali lo stesso Vico possedeva la predilezione, ma non ancora la penetrazione, quando compose il De antiquissima. Sicché la ragione piena di quell'interessamento si vede quando si consideri l'origine del Vico filosofo e si tenga presente il earattere della sua mente, antitetica allo spirito cartesiano. Il cartesianismo, tutto rivolto alle forme universalizzanti e astrattive, trascurava le individualizzanti; e tanto piú il Vico doveva essere attirato da esse come da un mistero. Il cartesianismo rifuggiva con orrore dalla selva selvaggia della storia; e il Vico s'internava bramoso in quella parte appunto della storia, nella quale, per cosí dire, è piú forte il sentore della storicità: nella storia che è piú lontana e diversa dalla psicologia delle eta colte. Il cartesianismo generalizzava questa psicologia a tutti i tempi e a tutti i popoli, e il Vico era portato a indagare nelle loro profonde differenze e opposizioni i modi di sentire e di pensare delle varie età.

Lo sforzo grande che bisognava fare, e ehe egli stesso fece, per riprendere, attraverso l' intellettualismo moderno, la coscienza della psicologia primitiva, è espresso dal Vico, dove parla delle « aspre difficultà * che gli era costata * la ricelca di ben venti anni », per * discendere da queste nostre umane nature ingentilite a quelle affatto fiere ed immani, le quali ei è affatto negato d'imaginare e solamente a gran pena ei è permesso d'intendere »; 0 , poeo diversamente, quando insiste sull'impossibilità - ora che le menti umane sono troppo ritirate dai sensi perfino presso il volgo, adusate ai tanti vocaboli astratti, assottigliate con l'arte dello scrivere, quasi spiritualizzate dalla pratica dei numeri - di entrare nella vasta immaginativa dei primi uomini, * le menti dei quali di nulla erano astratte, di nulla assottigliate, di nulla spiritualizzate, anzi tutte profondate nei sensi, tutte rintuzzate dalle passioni, tutte seppellite nei corpi s, e di formare 
l'idea, per es., della s natura simpatetica ». E quello sforzo, doloroso ma trionfante, che aveva dovuto compiere, era un'altra delle ragioni per le quali egli sentiva come * nuova $\gg$ la sua Scienza. Di questa infatti, ossia della ricerca sulla forma ideale e sull'epoca storica del certo, mancò (egli dice) tutta la greca filosofia. Platone l'aveva tentata invano nel Cratiio, perché gli era rimasta ignota la lingua dei primi legislatori, dei poeti eroi, tratto in inganno dalle formole emendate e ammodernate che le leggi erano venute rivestendo via via in Atene. In un errore analogo erano caduti tra i moderni Giulio Cesare Scaligero, Francesco Sanchez e Gaspare Schopp, che presero a spiegare le lingue coi principî della logica, e della logica aristotelica, sorta tanti secoli dopo le lingue. E il Grozio, il Selden, il Pufendorf e gli altri scrittori del diritto naturale meditarono anch'essi sulla natura umana ingentilita dalla religione e dalle leggi, sicché ritrassero il corso storico cominciando dalla meta in giú; ossia si fermarono sull'intelletto e ignorarono la fantasia, sulla volonta moralmente disciplinata e trascurarono la selvaggia passione. Egli stesso, il Vico, se col prendere a indagare l'antichissima sapienza italiana aveva dato segno del suo interessamento per quel problema, si era, per altro, sviato nella ricerca, seguendo le orme dell'autore del Cratilo.

Sotto l'aspetto filosofico, la Scienza nuova, per questa preponderanza che vi ha l'indagine delle forme individualizzanti e in ispecio della fantasia (la dottrina dei primi popoli come poeti e del loro pensare per caratteri poetici è, dice il Vico, « la chiave maestra * dell'opera), si potrebbe non troppo paradossalmente definire una filosofia dellospirito con particolare riguardo alla filosofia della fantasia, cioc̀ all'Estetica.

L'Estetica è da considerare veramente una scoperta del Vico: sia pure con le riserve onde s'intendono sempre 
circondate tutte le determinazioni di scoperte e di scopritori, e quantunque egli non la trattasse in un libro speciale, né le desse il nome fortunato col quale doveva battezzarla, qualche decennio piú tardi, il Baumgarten. Del resto, giova notare che nella terminologia della Scienza nuova s'incontra un nome simile ad alcuno degli equivalenti che il Baumgarten passava in rassegna per l'Estetica: quello di Logica poetica. Ma, in fondo, il nome importa poco, e assai importa la cosa; e la cosa è che il Vico espose una idea della poesia, che era a quei tempi, e doveva rimanere per un pezzo ancora, un'ardita e rivoluzionaria novità. Persisteva allora la vecchia idea praticistica o pedagogica, che dalla tarda antichità, attraverso il Medioevo, si era trapiantatá e radicata nel Rinascimento, della poesia come ingegnoso rivestimento popolare di sublimi concetti filosofici e teologici; e, accanto a questa, sebbene in grado minore, l'altra che la considerava come prodotto o strumento di svago e di voluttà. Queste concezioni avevano alterato perfino il senso originale del trattato aristotelico della Poetica, nel quale venivano introdotte e poi lette come se effettivamente Aristotele le avesse pensate e scritte. Né il cartesianismo le rettificò, ma piuttosto (com'era da aspettare, data la sua generale tendenza) attenuò e annullò l'oggetto medesimo di quelle definizioni, come cosa di nessuno o di traseurabile valore. In un tempo in cui si cercava di ridurre a forma matematica la metafisica e l'etica, in cui si dispregiava l'intuizione del concreto, si escogitavano una letteratura e una poesia atte a diffondere la scienza nel volgo o nel bel mondo, s'iniziavano tentativi per foggiare lingue artificiali logiche piú perfette di quelle storiche e viventi, e perfino si teneva possibile di stabilire regole per comporre arie musicali senza essere musicisti e poemi senza essere poeti; - in codesto ambiente distratto, gelido, nenico, beffardo, solo un miracolo sembra potesse risvegliare 
una diversa e opposta coscienza, una coscienza calda e veemente di quel che sia veramente la poesia e della sua originale funzione; e questo miracolo fu compiuto dallo spirito tormentato, agitato e scrutatore di Giambattista Vico.

Il quale criticd tutt'insieme le tre dottrine della poesia, come esornatrice e mediatrice di verità intellettuali, come cosa di mero diletto, e come esercitazione ingegnosa di cui si possa senza danno far di meno. La poesia non è sapienza riposta, non presuppone la logica intellettuale, non contiene filosofemi: i filosofi, che ritrovano queste cose nella poesia, ve le hauno ficcato dentro essi stessi, senza arvedersene. La poesia non è nata per capriccio di piacere, ma per necessita di natura. La poesia tanto poco è superflua ed eliminabile che, senza di essa, non sorge il pensiero: è la prima operazione della mente umana. L'uomo, prima di essere in grado di formare universali, forma fantasmi; prima di riflettere con mente pura, avverte con animo perturbato e commosso; prima di articolare, canta; prima di parlare in prosa, parla in versi; prima di adoperare termini tecnici, metaforeggia, e il suo parlare per metafore è tanto proprio quanto quello che si dice " proprio». La poesia, non che essere una maniera di divulgare la metafisica, è distinta e opposta alla metafisica: l'una purga la mente dai sensi, l'altra ve la immerge e rovescia dentro; l'una è tanto piú perfetta quanto piú s'innalza agli universali, l'altra quanto più si appropria ai particolari; l'una infievolisce la fantasia, l'altra la richicde robusta; quella ci ammonisce di non fare dello spirito corpo, questa si diletta di dare corpo allo spirito; le sentenze poetiche sono composte di sensi e passioni, quelle filusofiche di rifiessioni, che, usate nella poesia, la rendono falsa e fredda: non mai, in tutta la distesa dei tempi, uno stesso uomo fu insieme grande metafisico e grande poeta. Poeti e filosofi possono dirsi gli uni il senso, gli altri l'intel- 
letto dell'umanità; $e$ in tale significato è da ritenere vero il detto delle scuole che « niente è nell'intelletto che prima non sia nel senso ». Senza il senso, non si da intelletto; senza poesia, non si dà filosofia né civiltà alcuna.

Quasi piú miracoloso di questa concezione della poesia è che il Vico intravedesse la qualitả genuina del linguaggio: problema non meglio risoluto e assai meno agitato e investigato dalla filosofia antica e nuova, fino a quel tempo. Il linguaggio si soleva, a volta a volta, o confonderlo con la logicità o abbassarlo a semplice segno estrinseco e convenzionale o, per disperazione, dichiararlo di origine divina. Il Vico intese che l'origine divina era, in questo caso, un rifugio da pigri, e che il linguaggio non è né logicità né arbitrio, e, al pari della poesia, non è prodotto né di sapienza riposta né di placito o convenzione. Il linguaggio sorge naturalmente: nella prima forma di esso, gli nomini si spiegarono a con atti muti », ossia per cenni, e a con corpi aventi naturali rapporti alle idee che volevano significare », ossia per oggetti simbolici. Ma, anche per i linguaggi articolati e per le lingue volgari, « con troppo di buona fede », cioè con iscarso accorgimento, è stato ricevuto da tutti i fllologi che essi significhino a placito; laddove, per le anzidette origini, dovettero significare naturalmente, e ogni parola volgare cominciare certamente da un singolo individuo di una nazione e provenire dal linguaggio primitivo per cenni e per oggetti. Nel latino, come nelle altre lingue, si osserva che quasi tutte le voci sono formate per proprieta naturali o per trasporti; e il maggior corpo delle lingue tutte, presso tutte le nazioni, è costituito dalla metafora. La diversa opinione deriva dall'ignoranza dei grammatici, i quali, abbattutisi in gran numero di vocaboli che offrono idee confuse e indistinte, non sapendone le origini onde furono un tempo luminose e distinte, escogitarono, per darsi pace, la dottrina 
della convenzione, e vi trassero Aristotele e Galeno, armandoli contro Platone e Giamblico. La grave difficolta, che si suole mettere innanzi contro l'origine naturale del linguaggio e in favore della convenzione, la diversita delle lingue volgari secondo i popoli, si scioglie col considerare che i popoli, per la diversità dei climi, temperamenti e costumi, guardarono le melesime utilità o necessità della vita sotto aspetti diversi, e percio produssero lingue diverse; com'è comprovato altresi dai proverbî, che sono massime di vita umana sostanzialmente identiche, eppure spiegate in tanti diversi modi quante sono state e sono le nazioni. Singolarmente importante è poi l'insistenza onde il Vico professa di avere ritrovato le vere origini delle lingue « nei principî della poesia»: con che viene, per una parte, riasserita l'origine spontanea e fantastica del linguaggio, e dall'altra, se non per esplicito, certo per implicito, si tende a sopprimere la dualità di pocsia e linguaggio.

Nei quali principî della poesia il Vico ritrova non solamente l'origine delle lingue, ma anche quella delle lettere o scritture, dichiarando errore di grammatici la separazione fatta tra le due origini, che sono congiunte per natura e che come tutt' una cosa si presentano nella lingua primitiva mutola, per cenni e per oggetti. La sapienza riposta e la convenzione non hanno luogo neppure qui: i geroglifici non furono un ritrovato di filosofi per nascondervi dentro i misteri delle loro grandi idee, ma comuni e naturali necessità di tutti i primi popoli; e solamente le scritture alfabetiche nacquero tra $\mathrm{i}$ popoli gia inciviliti per effetto di libera convenzione. In altri termini, il Vico viene a distinguere, sia pure in modo confuso, nelle cosi dette scritture quella parte che è propriamente scrittura e percio convenzione, dall'altra che è invece diretta espressione, e percio linguaggio, favola, poesia, pittura. Caratteristica di queste scritture espressive o linguaggi è l'inseparabilita 
del contenuto dalla forma; la loro ragione poetica è tutta qui: che la favola e l'espressione siano una cosa stessa, cioè una metafora comune ai poeti e ai pittori, sicché un mutolo senza espressione verbale possa dipingerla. Il Vico arreca in esempio di esse alcuni aneddoti tradizionali, come le cillque "parole reali " (la ranocchia, il topo, l'uccello, il dente d'aratro e l'arco da saettare), che Idantura, re legli Sciti, mandò in risposta a Dario che gli aveva intimato guerra; e l'apologo degli alti papaveri che re Tarquinio svolse innanzi agli occhi dell'ambasciatore di suo liglio Sesto circa il modo di domare Gabî: - procedimenti espressivi non diversi da costumanze che si osservano ancora presso popolazioni selvagge e presso i volghi; e poi, altresí, le imprese, le bandiere, gli emblemi delle medaglie e monete. Una frivola favoletta, che rimpicciolisce e calunnia l'ufficio vero delle imprese, narra come esse venissero inventate nei tornei di Germania, qual costume di galanteria, dai garzoni che gareggiavano per meritare l'amore delle nobili donzelle. Ma le imprese, nel Medioevo, furono cosa seria, come a dire la scrittura geloglifica di quell'età: un parlare muto, che suppliva la povertà dei parlari convenuti o delle scritture alfabetiche; c solamente piú tardi, nei tempi colti, diventarono gioco e diletto, si convertirono in imprese galanti ed erudite, le quali bisogna animare coi motti, perché, ora, hanno significazioni solamente analoghe, laddove quelle primitive e naturali erano mutole e tuttavia parlavano senza bisogno cl'interpetri. In questa schietta naturalitả perdurano nei tempi colti alcune di tali forme espressive; per es., le insegne o bandiere, che sono una certa lingua armata, con la quale le nazioni, come prive di favella, si fanno intencere tra loro nei niaggiori affari del diritto naturale delle genti, nelle guerre, alleanze e commerci.

Cosí, al lume del concetto estetico pensato dal Vico, 
poesia, parole, metafore, scritture, simboli figurati, tutto si rischiara di lampi e dà guizzi di vita: cose grandi e cose piccole, l'epos e l'araldica. La dottrina delle forme fantastiche riceve un avviamento nuovo affitto nella storia delle idee; perché se il Vico si oppone coi suoi concetti alle scuole del suo tempo e specie alla cartesiana, nemmeno poi annoda e ripiglia altra scuola o tradizione piú o meno remota. Egli stesso sente la propria opposizione come diretta non contro una scuola particolare, ma contro tutte quelle che, nei secoli, avevano formolato dottrine sull'argomento. Circa la poesia dice che egli « rovescia » tutto ció che se ne era pensato da Platone e poi da Aristotele via via fino ai recenti Patrizzi, Scaligero e Castelvetro, i quali si perderono in inezie tali * che fa vergogna fin riferirle (il Patrizzi faceva nascere la poesia dai canti degli uccelli e dal sibilo dei venti!). Circa le lingue, il suo intendimento non era rimasto soddisfatto né da Platone né dai moderni Wolfango Lazio, Scaligero e Sanchez. Circa le lettere, rifiutata l'origine divina che era sostenuta dal Mallinkrot e da Ingevaldo Elingio, o (che valeva il medesimo) interpetratala a suo modo, da saggio per iscandalo delle vane opinioni, incerte, leggiere, sconce, boriose e ridevoli, che le facevano provenire dai Goti e per essi da Adamo e dalla personale comunicazione di Dio, o piú direttamente dal paradiso terrestre, o da un gotico Mercurio inventore. Cirea le imprese, infine, osserva che i tanti che ne avevano composto trattati, non ne avevano inteso nulla, e, solo per caso e indovinando, lasciavano trapelare un sentore della verità col chiamarle * croiche ». In realtà, sarebbe difficile assegnare veri e proprî precedenti ai concetti estetici vichiani, e tutt'al piú si potrebbero ritrovarne vaghe suggestioni in certe sparse sentenze che egli raceoglic; qualche stimolo piú prossimo nelle dispute secentesche sulle differenze tra intelletto e iugegno, ragione e immaginativa, 
dialettica e rettorica ${ }^{2}$; e qualche riscontro di particolari estrinseci, come nei ravvicinamenti fatti da qualche retore di quel tempo (il Tesauro) delle arguzie rettoriche parlate con le arguzie tigurate.

Senonché quei concetti, nati da cosi possente getto di originalita, non appena dai loro lineamenti generali si passi alle determinazioni particolari, dall'idea o ispirazione originaria agli svolgimenti effettivi, si vedono come turbarsi, ondeggiare, barcollare. Lasciamo da parte le varie successive opinioni che il Vico tenne, e che si legano al processo storico del suo spirito, sulla poesia, sulla lingua o sulla metafora, - dalle orazioni accademiche e poi dal De ratione e dal De antiquissima al Diritto universale, e ancora da questo alla prima, e dalla prima alla seconda Scienza nuova: indagine che potrebbe porgere argomento a un'apposita dissertazione e che non entra nel quadro della nostra esposizione. Ma, anche nella forma ultima del suo pensiero estetico, coesistono dottrine contradittoric. Egli non sta pago a dire, come ha detto, che la forma poetica è la prima operazione della mente, che essa è costituita da sensi di passione, è tutta fantastica, priva di concetti e di riflessioni; ma aggiungerà che la poesia, diversamente dalla storia, rappresenta il vero nella sua idea ottima, e compie percio quella giustizia e attribuisce quel premio e quella pena che spetta a ciascuno e che non sempre si ottiene nella storia, dominata sovente dal capriccio, dalla necessita e dalla fortuna. Dirà ancora che la poesia ha per suo fine l'animazione dell'inanimato, essendo il piú sublime lavoro di essa indirizzato a dare vita e senso alle cose insensate. Dira che la poesia non è altro che imitazione, e che i fanciulli, i quali valgono assai nell'imitare, sono poeti, e che i popoli primitivi, fanciulli del genere umano, furono in-

1 Si veda il capo 3.0 della parte storica della mia Estetica. 
sieme sublimi poeti. Dirà che la poesia ha per propria materia l'impossibile credibile, com'è impossibile che i corpi siano menti e pure fu creduto che il cielo tonante fosse Giove, onde i poeti non si esercitarono in altro maggiormente che nel cantare i prodigi compiuti dalle maghe per opera d'incantesimi. Dirà che la poesia è nata da inopia, ossia che è un effetto d'infermità dello spirito; perché l'uomo rozzo e di debole cervello, non potendo soddisfare il bisogno che prova del generale e dell'universale, foggia a sostituzione i generi fantastici, gli universali o caratteri poetici; e che, per conseguenza, il vero dei poeti e il vero dei filosofi sono lo stesso, questo astratto e quello rivestito d'immagini, questo una metafisica ragionata e quello una metafisica sentita e immaginata, confacente all'intendimento popolaresco. Parimente da inopia, cioè dall'incapacita ad articolare, sarebbe nato il canto, e percio i muti e gli scilinguati escono in suoni che sono canti; c dall'incapacità a significare le cose in modo proprio, le metafore. Dira, infine, che lo scopo della poesia è d'insegnare al volgo l'operare virtuosamente. - In questi detti sono accennati i piú diversi concetti sulla poesia, alcuni conciliabili con la dottrina fondamentale, ma proposti senza mediazione e percio effettivamente non conciliati; altri, affatto inconciliabili. Il Vico potrebbe essere, a volta a volta, sul fondamento di singoli testi, presentato come sosteuitorc dell'estetica moralistica, pedagogica, astratta e tipeggiante, mitologica, animistica, e via discorrendo. E se non ricasca nelle vecchie teorie che egli aborriva, e se non si dissipa tra gli errori nuovi che precorreva, si deve al fatto che su tutte quelle varieta e incoerenze sormonta costante il pensicro che la poesia è la prima forma della mente, anteriore all'intelletto e libera da riflessione e raziocinî. 
Come non seppe, valendosi del suo principio capitale, seeverare e accordare gli altri che circa la natura della poesia esistevano nella tradizione scientifica o erano stati da lui escogitati, cosí non riusci a liberarsi dalla tirannia delle classificazioni empiriche, vecchie e nuove. In cambio, si sforzò di filosofarle, e tentò di dedurre serialmente le diverse forme della poesia, epica lirica drammatica; del verso e del metro, spondaico giambico prosastico; del parlare figurato, metafora metonimia sineddoche ironia; delle parti del discorso, onomatopee interiezioni pronomi particelle nomi verbi, modi e tempi del verbo (al qual proposito richiama perfino un caso di afasia da lui osservato in Napoli in persona di « un nomo onesto tócco da grave apoplessia, il quale mentova nomi e si è dimenticato affatto de' verbi »); delle scritture, geroglifiche simboliche alfabetiche; delle lingue secondo la loro crescente complessità, che va dalle parole monosillabiche alle composte e dalla prevalenza di voeali e dittonghi alla prevalenza delle consonanti. In questi tentativi disseminò dappertutto interpetrazioni nuovo e parzialmente vere di fatti particolari; ma non giunse, e non poteva, a sistemazione scientifica. E neppure vide chiaro nella relazione della poesia con le altre arti, che talora unificò con quella, come quando considera intrinsecamente identiche pittura e poesia, e viene notando analogie tra la poesia e la pittura del Medioevo; e, tal'altra, stranamente separò, come quando pretende che la delicatezza delle arti sia frutto delle filosofie e che delicatissimo siano pittura, scultura, fonderia e intaglio, perché debbono astrarre le superficie dai corpi che imitano.

Queste incoerenze ed errori, che abbiamo passati in rapida rassegna, se in parte derivano da scarsa capacita di distinzione e di elaborazione, per un'altra e maggiore parte si riportano piú direttamente al già chiarito vizio fondamentale clue è nella struttura della Scienza nuova; 
e qui, propriamente, allo scambio fatto dal Vico tra il concetto filosofico della forma poetica dello spirito e il concetto empirico della forma barbarica della civilta. * Talché (egli stesso dichiara) questa prima età del mondo si può dire con verità tutta occupata d'intorno alla prima operazione della mente ». Ma la prima età del mondo, essendo costituita da uomini in carne ed ossa e non da categorie filosofiche, non poté essere occupata intorno a un a sola operazione della mente. Quest' una poteva, come si suol dire, prevalere (e la parola stessa scopre il carattere quantitativo e approssimativo del concetto); ma tutte le altre dovevano essere in atto insieme con lei, la fantasia e l'intelletto, la percezione e l'astrazione, la volontà e la moralità, il cantare e il numerare. A siffatta evidenza il Vico non poteva sottrarsi, epperò in quella fase di eiviltà introdusse non solo il poeta, ma anche il teologo, il fisico, l'astronomo, il paterfamilias, il guerriero, il politico, il lcgrislatore; senonché le attivita di tutti costoro volle considerare e chiamare poetiche, con metafora tratta dall'asserita prevalenza della forma fantastiea dello spirito, e il complesso di esso sapienza poetica. Il carattere metaforico della denominazione è accusato, o balza agli occhi, in alcuni luoghi caratteristici; come dove le « arti », ossia le arti meccaniche, produttrici pratiche di oggetti per gli usi della vita, sono definite e poesic in certo modo reali », e l'antico diritto romano, per l'abbondanza delle formole e cerimonie onde si riveste, è detto « poema drammatico serio ». Ma le metafore sono pericolose, quando, come nel caso della Scienza nuova, trovano terreno favorevole alla loro conversione in concetti; e, infatti, l'eta storica barbarica, metaforeggiata come sapienza poetica, non tardo a trasformarsi, presso il Vico, nell'eta ideale della poesia, conferendo a quest'ultima tutte le proprie attribuzioni. Cola erano teologi, e la poesia fu considerata dal 
Vico come teologia, sebbene fantastica; educatori, e fu fatta educatrice, sebbene di volgo; sapienti di cose fisiche, e fu fatta sapienza, sebbene di fisica immaginaria. E poiché quei barbari non potevano non pensare per concetti, rozzi che questi fossero e involti nelle immagini, $i$ fantasmi della poesia, individuati, singolarizzati, le sentenze di essa sempre corpulente, si falsificarono in universali fantastici, che sarebbero qualcosa di mezzo tra l'intuizione, che è individualizzante, e il concetto, che universalizza: la poesia, che doveva rappresentare il senso, lo schietto senso, rappresentò invece il senso gia intellettualizzato, e il detto che niente si trova nell'intelletto che non sia già nel senso, acquistò il significato che l'intelletto è il senso stesso, schiarito, o il senso l'intelletto stesso, confuso; onde non si ebbe piú bisogno dell'aggiunta cautela: * nisi intellectus ipse ». Per converso, la civilta barbarica divenne come una mitologia o allegoria della ideale età poetica; e i primi popoli furono trasformati in moltitudini di * sublimi poeti $\gg$; come poeti furono fatti (nella ontogenesi corrispondente a tale filogenesi) perfino i fanciulli. Il concetto dell' universale fantastico come anteriore all'universale ragionato concentra in sé la duplice contradizione della dottrina; perché all'clemento fantastico dovrebbe essere congiunto in quella formazione mentale l'elemento dell' universalità, il quale, per sé preso, sarebbe poi un vero e proprio universale, ragionato e non fantastico: donde una petitio principii, per la quale la genesi degli universali ragionati, che dovrebbe essere spiegata, viene presupposta. E, d'altro canto, se l'universale fantastico s' interpetrasse come purificato dell'elemento universale e logico, cioè come mero fantasma, la coerenza si ristabilirebbe certamente nella dottrina estetica; ma la sapienza poetica o civiltà barbarica verrebbe mutilata di una parte essenziale del suo organismo, perché privata di ogni sorta di concetti, e, per dir cosí, disossata. 
Per risolvere la contradizione conveniva dissociare poesia e sapienza poetica; del che, in veritá, s'incontra qualche accenno presso il Vico. Egli confessa talvolta, quasi involontariamente, la non corrispondenza tra la categoria filosofica e il tipo sociale, e per quest'ultimo è costretto a ricorrere ai «press'a poco», e ai «piú o meno». Gli accade di dire, per es., che gli uomini primitivi erano \& nulla o assai poco ragione e tutti robustissima fantasia », \& quasi tutti corpo e quasi niuna riflessione »; ovvero, dopo avere distinte con filosofiche pretese tre lingue degli dèi, degli eroi e degli uomini, osserverà che * la lingua degli dèi fu quasi tutta muta e pochissimo articolata; la lingua degli eroi mescolata egualmente di articolata e di muta; la lingua degli uomini quasi tutta articolata e pochissimo muta ». La favella poetica (ammette ancora) sopravvive alla sapienza poetica e scorre per lungo tratto dentro il tempo istorico o eta civile, come (dice con magnifica immagine) * i grandi rapidi fiumi si spargono molto dentro il mare e serbano dolci l'acque portatevi con la violenza del corso ». Anche nei tempi moderni non si può dismettere il parlare fantastico, $c$ \& per ispiegare i lavori della mente pura ei han da soccorrere i parlari poetici per trasporti de' sensi ». La poesia non sembra che sia finita con la fine della barbarie, perché pur nei tempi civili sorgono poeti; e che quelli della prima epoca fossero fantastici per natura, e i nuovi tali si facciano per arte ed industria - ossia, come il Vico vuole, con lo sforzarsi di perdere memoria delle parole proprie, di purgarsi delle filosofie, di riempirsi la mente di pregiudizî fanciulleschi o volgari, di rimettere la mente in ceppi costringendosi, tra l'altro, all' uso della rima, - queste restrizioni, del resto facilmente confutabili, si affaticano invano a sminuire l'importanza del fatto riconosciuto: che la poesia è di tutti i tempi, e non di quello solo barbarico; è 
una categoria ideale e non un fatto storico. Ma le restrizioni anzidette, come la rarità e la timidezza degli accenni ricordati, provano che il Vico non era in grado di eseguire la dissociazione tra poesia e sapienza poetica, impeditone dall' ibridismo del concetto e del metodo stesso della Scienza nuova.

Se, per altro, l'idea della poesia come pura fantasia, nonostante tutte le confusioni e incoerenze nelle quali si avvolge, non fosse rimasta salda nel fondo del pensiero del Vico, e non avesse operato, per cosí dire, nel sottosuolo della Scienza nuova, non sarebbe agevole, né forse possibile, intendere la concezione capitale che domina la sua filosofia dello spirito, e che è strettamente legata con quell'idea. Diciamo, la concezione dello spirito come sviluppo, o, per adoperare la terminologia propria del Vico, come corso o spiegamento: concezione la quale, pur senza espressa contrapposizione, superava quella ordinaria, limitantesi quasi esclusivamente a enumerare e classificare le facoltà dello spirito. La dottrina degli universali fantastici come spontanee formazioni mentali, universali rozzi ma forniti di un motivo di vero, era certamente bastevole come strumento per debellare l'empirica teoria che faceva sorgere le civilta da un'alta e ragionata saggezza ordinatrice, opera personale di Dio o di uomini sapienti, sorti non si sa come e piovuti non si sa donde. Il Vico poneva chiaro il dilemma delle due e non piú guise di spiegare l'origine della civiltả: o nella riflessione di uomini sapienti, ovvero in un certo senso e istinto umano di uomini bestioni; e si risolveva per la seconda ipotesi, per $i$ - bestioni * che via via si erano fatti uomini; cioè per il pensiero che si evolve dall'universale fantastico a quello ragionato, per l'assetto sociale che procede via via dalla forza all'equità. Ma era quella concezione bastevole per fondare la storia ideale o filosofia dello spirito? Nella fi- 
losofia dello spirito, essa si sarcbbe tradotta in qualcosa di simile, se non d'identico, alla dottrina che, per effetto del cartesianismo e anche di una certa tal quale rinascita che ebbe la scolastica di Duns Scotus, correva ai tempi del Vico, e secondo cui la vita dello spirito si esplicava nei gradi successivi del concetto oscuro, confuso, chiaro e distinto: il Leibniz, com'è noto, fece argomento di speciale studio le percezioni oscure e confuse, le «petites perceptions ». Dottrina nel suo intrinseco intellettualistica, perché i concetti, confusi e oscuri che fossero, erano pur sempre concetti; e impotente perciò a dare ragione, nonché della poesia, neppure dello sviluppo spirituale, che non può intendersi nella sua dialettica quando sia costituito di differenze meramente quantitative, le quali, in realtà, non sono differenze ma identità e perciò immobilità; e, infatti, tutto quell'indirizzo fu, insieme, antiestctico e statico, privo di una vera dottrina della fantasia e di una vera dottrina dello sviluppo. 11 pensiero del Vico è, invece, avverso all'intellettualismo, simpatico alla fantasia, tutto dinamico ed evolutivo; lo spirito è, per lui, un eterno dramma; e, poiché il dramma vuole tesi e antitesi, la sua filosofia della mente è impiantata sull'antinomia, cioè sulla reale distinzione e opposizione di fantasia e pensiero, poesia e metafisica, forza ed equita, passione e moralita, per quanto egli sembri talvolta, per le ragioni gia note, disconoscerla o, piuttosto, per quanto venga talvolta a ingarbugliarla con indagini e dottrine empiriche e con determinazioni storiche. 
. , 
LA FORMA SEMIFANTASTICA DEL CONOSCERE

(IL Mito E LA RELigione)

Anche la dottrina del Vico sul mito, se è non meno originale e profonda di quella circa la poesia, non è del tutto limpida, perché le relazioni tra poesia e mito sono cosí strette che l'ombra gettata sull' una deve necessariamente stendersi in qualche modo sull'altro.

Proseguendo a indagare, come abbiamo fatto sin qui e faremo sempre nel séguito, lo stato delle cognizioni ai tempi del Vico secondo le varie discipline e problemi che egli prese a trattare, ricorderemo in breve, circa gli studî sulla mitologia, come tra il Cinque e il Seicento non solamente si mettessero insieme grandi compilazioni lettelarie di miti (delle quali gia aveva dato esempio, nel Trecento, il Boccaccio), ma venissero dottamente propugnate le due teorie esplicative gia note all'antichità classica e mon ignote del tutto al Medioevo: la teoria del mito come allegoria di veritá filosofiche (morali, politiche e via discorrendo), e quella del mito come storia di personaggi effettivamente esistiti e di avvenimenti accaduti, adornate dall'immaginazione che divinizzava gli eroi (evemerismo). L'allegorismo ispirava, tra l'altre, l'opera di Natale Conti, Mythologice sive explanationis fabularum libri decem (1568) 
e il De sapientia veterum (1609) del Bacone; dove, per altro, quel sistema era proposto non senza qualche dubbio e con la espressa cautela che, se anche non valesse come interpetrazione storica, avrebbe potuto sempre mantenere il suo valore di moralizzazione (aut antiquitatem illustrabimus aut res ipsas). Il neoevemerismo cra rappresentato autorevolmente da Giovanni Leclerc (Clericus), l'erudito ginevrino-olandese verso cui tanta reverenza e gratitudine ebbe a professare il Vico per aver degnato di attenzione il suo Diritto universale, e del quale fece epoca, in materia mitologica, l'edizione della Teogonia esiodea; lo seguí tra gli altri il Banier, autore del libro: Les fables expliquées par. l'histoire (1735). Un terzo sistema, anch'esso non senza qualche precedente antico, derivava $i$ miti da popoli particolari, dagli egiziani o dagli ebrei, ovvero dall'opera di singoli filosofi e poeti inventori; e, quando non si risolveva in una pura e semplice ipotesi storica sulla formazione di alcuni o di tutti i miti trasmessi dall'antichità o non si riportava alla rivelazione divina, è chiaro che implicava la teoria che il mito sia non già una forma eterna, ma un contingente prodotto dello spirito, il quale, com'è nato una volta, cosí possa morire o sia già morto.

Il Vico si oppone risolutamente alla prima e alla terza scuola, all'allegorismo e alla dottrina della derivazione storica; e ricorda, per la prima, il trattato baconiano dal quale aveva tratto incentivo a meditare sull'argomento, ma ch'egli giudicava « piú ingegnoso che vero »; e per l'altra scuola, considerante i miti come storie sacre alterate e corrotte dai gentili e in particolare dai greci, il De theologia gentili (1642) del Vossio, la Demonstratio evangelica (1679) di aniele Huet, e il Phaleg et Canaan del Bochart. I miti o favole non contengono sapienza riposta, cioè concetti ragionati, avvolti consapevolmente nel velo della favola; e perciò non sono allegorie. L'allegoria im- 
porta che si abbia, da una parte, il concetto o significato, dall'altra la favola o involucro, e tra le due cose l'artifizio che le fa stare insieme. Ma i miti non si possono scindere in questi tre momenti, e neppure in un significato e un significante: i loro significati sono univoci. Importa altresí, quella teoria, che chi crede al contenuto, non creda alla forma; ma i creatori dei miti dettero ingenua e piena fede a quelle loro creazioni; e fintasi, per es., la prima fuvola divina, la piú grande di quante mai se ne finsero in appresso, Giove re e padre degli dèi e degli uomini in atto di fulminante, essi stessi che se lo finsero lo credettero, e con ispaventose religioni lo temerono, riverirono e osservarono. Il mito, insomma, non è favola ma storia, quale possono formarsela gli spiriti primitivi, e da questi è severamente tenuta come raceonto di cose reali. I filosofi che sorsero posteriormente, servendosi dei miti per esporre in modo allegorico le loro dottrine, ovvero illudendosi di ritrovarvele per quel senso di riverenza che si porta all'antichita tanto piú venerabile quanto piú oscura, ovvero stimando comodo di giovarsi di tale espediente per i loro fini politici, - e cosi Platone omerizzando e, nel tratto stesso, platonizzando Omero; - resero i initi favole, quali in origine non erano e intrinsecamente non sono. Onde è da dire che filosofi e mitologi furono piuttosto essi i poeti che immaginarono tante strane cose sulle favole, ladrove i poeti o ereatori primitivi furono i veri mitologi e intesero narrare cose vere dei loro tempi. - Per la medesima ragione, ossia per essere i miti parte essenziale della sapienza poetica o barbarica, e come tale spontanei in tutti i tempi e luoghi, non si pưo attribuirli a un singolo popolo che li avrebbe inventati e dal quale si sarebbero trasmessi agli altri, quasi ritrovato particolare di uomini particolari od oggetto di rivelazione.

Codesta dottrina, superante l'allegorismo e lo storiei- 
smo, è un altro aspetto della rivendicazione che il Vico compi delle forme conoscitive alogiche contro l'intellettualismo, il quale le negava appunto col presentarle ora come forme artificiali ora come prodotti accidentali o dovuti a cause soprannaturali. Né sembra accettabile l'opinione che aggrega il Vico all'indirizzo neoevemeristico, da lui in verità non combattuto espressamente e verso il quale presenta anche, se si vuole, alcune superficiali soniglianze, ma insieme con le somiglianze questa radicale diversità: che per lui le favole non sono alterazioni di storie reali né si riferiscono di necessita a individui reali, ma sono intrinsecamente verita storica, nella forma che la verita storica suol prendere nelle menti primitive.

Altra piú precisa determinazione circa la natura del mito il Vieo non dà né poteva, appunto perché essendo in lui ondeggiante il concetto stesso della poesia, egli non era in grado di segnare i limiti tra le due forme. Parlo, in genere, di poesia e di mito come di cose distinte, ma non fermò la distinzione. Eppure, il Vico si era bene imbattuto nel concetto che porge quel criterio distintivo, e l'aveva enunciato; senonché, in cambio di valersene per la dottrina del mito, ne aveva fatto una o alcune delle sue parecchie definizioni della poesia. Quel carattere poetico, quell'universale fantastico che, introdotto nell'estetica come principio esplicativo della poesia, dà origine a tante insuperabili difficoltà, è invece, per l'appunto, la definizione del mito, e come tale fornisce alla scienza della mitologia il vero principio che le bisogna. Se il. concetto del compiere grandi fatiche pel comune vantaggio non si sa staccare dall' immagine di un uomo particolare che abbia compiuto alcuna di quelle fatiche, quel concetto diventa il mito, per es., di Ercole; ed Ercole è insieme un individuo. che fa azioni individuali e uccide l'idra di Lerna e il leone nemeo o lava le stalle di Augia, ed è un concetto; come 
il concetto dell'operosita utile e gloriosa è un concetto ed è, insieme, Ercole: è un universale e un fantasma: un universale fantastico.

Anche quel subline lavoro, che il Vico diceva proprio della poesia, di dare vita alle cose inanimate, spetta non propriamente alla poesia ma al mito. Il quale, incorporando i concetti in immagini, ed essendo le immagini sempre qualcosa d'individuale, viene ad atteggiarli come esseri viventi. Cosí gli uomini primitivi, che non conoscevano la cagione del fulmine e perciò non ne possedevano la definizione fisica, erano tratti, miteggiando, a concepire il cielo come un vasto corpo animato, che - a somiglianza di essi medesimi quando erano in preda alle loro violentissime passioni, urlando, brontolando, fremendo, - parlasse e volesse dire qualche cosa. E del mito e non della poesia si deve riconoscere l'origine nell' « inopia», nella debolezza della mente e nella sua inadeguazione ai problemi che vuole risolvere, nella incapacità a pensare per universali ragionati e a esprimersi con termini proprî, onde sorgono gli universali fantastici e le metonimie e le sineddoche e ogni sorta di metafore. Le contradizioni, notate da noi nell'universale fantastico e che lo rendono inadatto a fondare la dottrina estetica, stanno perfettamente a posto nella dottrina del mito; il quale è, per l'appunto, questa contradizione: un concetto che vuol essere immagine e un'immagine che vuol essere concetto, e perciò un'inopia, anzi un'impotenza potente, un contrasto e una transizione spirituale, dove il nero non è ancora e il bianco muore. Infine, la sapienza poetica, cioè la teologia, fisica, cosmografia, geografia, astronomia e tutto il complesso delle restanti idee e credenze dei popoli primitivi, esposte dal Vico, erano effettivamente mito e, non, come egli dice, poesia, per la buona ragione ch'egli stesso adduce che quelle erano le loro storie; e la poesia è poesia e non istoria, neppure 
piú o meno fantasticata. Poesia, i pocmi omerici in quanto esprimerano i scntimenti c le umane aspirazioni della grecità; storia, gli stessi poemi omerici, in quanto erano cantati e ascoltati come racconti di fatti realmente accaduti : rue forme di prodotti spirituali che, se sembrano materialmente racogliersi in una stessa opera, non per ció s'identificano.

Tutto questo il Vico vede e non vede, o, meglio, ora intravede c ora travede e perciò non si puỏ dire che riesca a determinare veramente la distinzione e a risolvere il problema dei rapporti tra mito e poesia. Un altro importante e ancora assai dibattuto problema della scienza mitologica, se cioè il mito sia filosofia o storia, potrebbe credersi, inrece, da lui risoluto in modo netto; perché egli ripete molte volte che i miti contengono sensi storici, e non già filosofici, dei popoli primitivi; ma, in realtà, ove si faccia bene attenzione, si scorge che egli, nonché risolverlo, non se lo propone neppure. I sensi storici, che il Vico assevera, sono contrapposti non propriamente ai sensi filosofici in genere, ma « ai sensi mistici di altissima filosofia » a a sensi analogi », che i mitologi da lui criticati vi ritrovavano; cioè, da una parte ripetono la critica all'allegorismo e, dall'altra, combattono quel cattivo modo d'interpetrazione storica che trasferisce idee e costumi moderni ai popoli antichi. La sua teoria si concilia, a dir vero, alla pari con quella che avvicina il mito alla filosofia, e con l'altra che l'avvicina alla storia; con l'eclettica che ammette entrambi gli elementi, e con la speculativa, che li ammette altresí cntrambi ma per la ragione che filosofia e storia, cosí in sé medesime come nel mito, costituiscono, in fondo, una cosa sola e indivisibile.

Come «inopia», il mito deve essere superato. La mente umana - che agogna naturalmente di unirsi a Dio donde clla viene, cioè al vero Uno, e che non potendo per la 
esuberante natura sensuale dell' uomo primitivo esercitare lat facolta, sepolta sotto i loro sensi troppo vigorosi, di astrarre dai subietti le proprietà e le forme universali, si era finta le unita immaginarie, i generi fantastici o i miti, - nel suo successivo spiegarsi o esplicarsi risolve via via i generi fantastici in generi intelligibili, gli universali poetici in ragionati, e si libera dai miti. L'errore del mito passa cosí nella verità della filosofia. Il Vico conosce e adopera un concetto dell'errore, dell'error'e propriamente detto, nascente dalla volonta e non dal pensiero, il quale quanto a sé non erra mai (mens enim semper a vero urgetur quia nunquam aspectu amittere possumus Deum); dell'errore che consiste in vuote parole arbitrariamente combinate (verba autem sapissime veri vim voluntate mentientis eludunt ac mentem deserunt, immo menti vim faciunt et Deo obsistunt); dell'errore, insomma, che, per adoperare la sua efficacc descrizione, si ha quando gli nomini «mentro con la bocea dicono, non hanno nulla in lor mente, perocché la lor mente è dentro il falso, che è nulla». Ma sa anche che l'errore non è mai del tutto errore, appunto perché, non potendosi dare idee false e consistendo il falso soltanto nella sconcia combinazione delle idec, in esso è sempre il vero, e ogni favola ha qualche * motivo di verità . Perciò, lnngi dal disprezzare le favole, ne riconosce il valore quasi di embrione del sapere riposto o della filosofia che si svolgerà poi. I poeti (ossia, nel nuovo significato che assume nel Vico questa parola, i creatori dei miti) sono il senso (cioè, nel nuovo significato, la filosofia rudimentale e imperfetta); e i filosofi sono l'intelletto dell'umanità (vale a dire, la filosofia piú compiuta, che nasce dalla precedente). L'jdea di Dio si evolve a poco a poco dal Dio, che colpí la fantasia dell' nomo isolato, al Dio delle famiglie, divi parentum, al Dio della classe sociale o della patria, divi patrii, al Dio delle nazioni, fino a quel Dio "che a tutti è Giove», 
al Dio dell'umanità. Le favole destarono Platone a intendere le tre pene divine, che gli dèi solamente, e non gli uomini, possono infliggere: l'oblio, l'infamia e il rimorso; il passaggio per l' Inferno gli suggerí il concetto della via purgativa onde l'anima si purifica dalle passioni, e l'arrivo agli Elisi quello della via unitiva onde la mente va ad unirsi a Dio per mezzo della contemplazione delle eterne cose divine. Dalle somiglianze e metafore dei poeti Esopo trasse gli esempî e gli apologi con cui dette i suoi avvisi; e dall'esempio, che si fonda sopra un caso solo e soddisfa le menti rozze, si svolge l'induzione, che si vale di piú casi simili, quale l'insegnò Socrate con la dialettica, e successivamente il sillogismo, che Aristotele scoperse e che non regge senza un universale. Le etimologie delle parole svelano le verita intraviste dai primi uomini e deposte nel loro linguaggio; per es., cio che i filosofi moderni con gravi ragioni hanno dimostrato, che i sensi fanno essi le qualita chiamate « sensibili », è già adombrato nella parola «olfacere d della lingua latina, che implica il pensiero che l'odorato \& faccia * l'odore. Il Vico attribuisce tanta importanza a questa connessione tra universali poetici e universali ragionati, tra mito e filosofia, da essere tratto ad affermare che le sentenze dei filosofi, le quali non trovino precedente e riscontro nella sapienza poetica e volgare, debbano essere errate. Anzi, è questo un altro significato che egli assegna talvolta al rapporto tra filosofia e filologia: di una conferma reciproca tra sapienza volgare e sapienza riposta, conciliate entrambe nell'idea di una filosofia perenne dell' umanità.

Con la teoria del mito e del rapporto di esso con la filosofia il Vico ha dato, tutt'insieme, la sua teoria della religione e del rapporto tra religione e filosofia. Due pensieri circolano, a questo proposito, per entro la Scienza nuova: l'uno, che la religione nasca, nella fase della debo- 
lezza e dell'incultura, dal bisogno mentale di dare pace alla curiosita e d'intendere in qualche modo le cose della natura e dell' uomo (di spiegare, per es., il fulmine); I'altro, che la religione s' ingeneri negli animi pel terrore di colui che minaccia fulminando. E si potrebbero chiamare le due teorie, dell'origine teoretica e dell'origine pratica della religione; e poiché, conformemente alle dottrine del Vico, l'uomo è nient'altro che intelletto e volontà, è chiaro come, fuori di queste due origini, la religione non possa averne altre. Ora, lasciando da parte la religione nel significato pratico (della quale si discorrerà piú innanzi), la religione nel significato teoretico che cosa c̀ altro se non l'universale fantastico, l'animismo poetico, il mito? A essa si lega quell' istituto che il Vico chiama la divinazione, il complesso dei metodi coi quali si raceoglieva e interpetrava la lingua di Giove, le parole reali, i segni e cenni del Dio, finto nell' universale fantastico e ereato dall'immaginazione animatrice. $\mathrm{E}$ come dal mito procede la scienza e la filosofia, cosí, parimente, dalla divinazione la conoscenza delle ragioni e cause, la previsione filosofica e scientifica.

Il Vico, a questo modo, si liberava dal pregiudizio che cominciava a prevalere al suo tempo (si ricordino la storia degli oracoli antichi del Van Dale, resa popolare dal Fontenelle, e il libro gia citato del Banier), e tanta efficacia ebbe per un secolo ancora, delle religioni come <impostura d'altrui », quando erano invece (egli dice) nate da " propria credulità ". Colui che non ammetteva l'origine artificiale dei miti, non poteva ammetterla neppure delle religioni. Ma come egli rifiutava altresí l'origine sopranna-. turale o rivelata dai miti, cosí nello stesso atto pronunziava né piú né meno che l'origine naturale, anzi umana, delle religioni; e, quel che piú specialmente è da notare, la riponeva in una forma inadeguata dello spirito, nella 
forma semifantastica, che è il mito. Né bisogna fare caso di qualclic suo breve detto incidentale, che sembra in contrasto con questa teoria; come là dove dice che la religione precede non solo le filosofie ma il linguaggio stesso, il quale suppone la coscienza di qualcosa di comune tra gli uomini: equivoci derivanti dalla solita perplessità metodica e da. abito di poca chiarezza. L'identificazione della religione col mito, e l'origine umana delle religioni, non solo è insistentemente espressa, ma è essenziale a tutto il sistema del Vico. Origine umana, che non esclude, nelle parole di lui, un diverso concetto di religione: la religione rivelata, e percio di origine soprannaturale. Egli, infatti, pone sempre, da un canto, la teologia poetica, che è mitologia, e la. teologia naturale, che è metafisica o filosofia; e, dall'altro, la teologia rivelata. Ma quest'ultimo concetto c̀ ammesso da lui, non perché si leghi ai precedenti e tutti derivino da un principio comune, si bene semplicemente perchè il Vico afferma gli uni e afferma l'altro. L'origine umana, la teologia poetica, di cui è séguito la teologia metafisica, è quella che vale per l'umanità gentilesca, ossia per l'umanita intera, fatta eccezione del popolo ebreo che è privilegiato dalla rivelazione. Per quali motivi il Vico serbassc questo dualismo, e sopra quali contradizioni pungenti fosse a cagione di esso costretto ad adagiarsi, anclie questo si vedra piú oltre, e a suo luogo. Ma appunto perché quel dualismo rimase in lui senza mediazione, noi dobbiamo, esponendo il suo pensiero, tenere fermo ciascuno dei due termini del dualismo, e, per ora, l'origine meramente umana: la religione quale prodotto del bisogno teoretico dell'nomo giacente in condizioni di relativa povertà mentalc. Concetto che lia rapporti solanente indiretti con quello bruniano della religione come cosa necessaria alla moltitudine rozza e poco sviluppata, e con quello campanelliano della religione naturale o perpetua, 
eterna filosofia razionale coincidente col cristianesimo spogliato dai suoi abusi; e che ha rari e deboli riscontri negli scrittori del tempo, i quali, anche quando vi accennano di passaggio, l'intendono in modo superficiale e lo presentano senza nessuna coerenza con le altre loro idee: battono sulla religione in quanto ignoranza e trascurano la sapienza di quella ignoranza, la religione come verita. 
. 


\section{LA COSCIENZA MORALE}

Lue altre dottrine del Vico di ragion teoretica, cioè di logica della filosofia, delle scienze fisiche e matematiche e delle discipline storiche, sono state già esposte nell'esporre la sua gnoseologia, e si desumono quasi tutte dai primi scritti, perché nella Scienza nuova la fase della «mente tutta spiegata» appare, piú che altro, come un limite della ricerca. Soltanto giova notare che il Vico tocea altresí il problema del rapporto tra poesia e storia, ma, sempre a causa dell'indistinzione tra filosofia e scienza sociale, non gli riesce di risolverlo pienamente. Sotto un aspetto, sembra a lui che la storia sia anteriore alla poesia, perché questa, dice, presuppone la realtà e contiene una «imitazione di piủ»; sotto un altro aspetto, che la poesia costituisca la forma prima, perché presso i popoli primitivi la loro storia è la loro poesia e i primi storici sono i poeti. A ogni modo, egli insiste sull'elemento poetico, intrinseco alla storia; e di Erodoto, padre della greca storia, osserva che non solo i libri di lui sono ripieni la piú parte di favole, ma « lo stile ritiene moltissimo dell'omerico, nella qual possessione si sono mantenuti tutti gli storici che sono venuti appresso, i quali usano una frase mezza tra la poetica e la volgare s: "verba ferme poëtarum ", come ripete altrove facendo suo un detto di Cicerone. 
Né si trovano svolti particolarmente nel Vico i rapporti fra teoria e pratica, intelletto e volonta, benché dappertutto egli suggerisca il pensiero generale che come in Dio intelletto e volontà coincidono, similmente nell' uomo, immagine di Dio; onde la mente o spirito non è divisa in un pensiero e in una volontà, in un pensiero che proceda per un verso e in una volontà che proceda per un altro, ma pensiero e volontà si compenetrano e formano un tutto solo: concezione assai superiore a quella della filosofia del suo tempo, cioè del leibnizianismo, in cui persisteva il concetto dell'arbitrio divino, e percio dell'irrazionalità. Un altro suo e singolare pensiero importerebbe invece, per chi concluda frettolosamente, la precedenza della pratica sulla teoria; perché il Vico dice che i filosofi pervengono ai loro conectti mercé l'esperienza delle istituzioni sociali c delle leggi nelle quali gli uomini si accordano come in qualcosa di universale, e che Socrate e Platone, per es,, presuppongono la democrazia e i tribunali ateniesi. Ma questa successione delle religioni che generano le repubbliche, delle repubbliche che generano le leggi, delle leggi che generano le jdee filosofiche, e che egli chiama « una particella della storia della filosofia narrata filosoficamente », è, appunto, teoria d'importanza non filosofica ma sociologica.

Per quel che concerne le dottrine di ragion pratica, delle quali ora entriamo a trattare, potrebbe parere che il Vico, diversamente che in quelle di ragion teoretica, non sia in recisa opposizione alle jdee del suo tempo, ma anzi si ricolleghi proprio a un movimento del suo tempo: alla scuola del diritto naturale. Il capo della scuola, l'iniziatore del movimento, Ugo Grozio, era da lui chiamato uno dei suoi quattro autori, insieme con Platone, in cui trovava appagata la sua brama di una filosofia idealistica, col Bacone che gli aveva fatto sorgere in mente l'idea di una scienza positiva e storica delle societa, e con Tacito, che 
vedremo piú innanzi qual servizio gli rese o il Vico credette di averne ottenuto. E insieme col Grozio ricorda perpetuamente gli altri principali autori del diritto naturale, il Selden e il Pufendorf, trascurando gl'innumerevoli loro seguaci, che considera, piuttosto che autori di scienza, sempliei « adornatori » del sistema groziano.

Il ricollegamento, in un certo senso, è evidente e confessato e professato dallo stesso Vico; ma anche è indubitabile che egli non aderi semplicemente a quella scuola, e neppure la continuò al modo di chi serbi i concetti generali e direttivi, e svolga o corregga i particolari. La continuò solamente in significato dialettico, cioè in quanto ne ebbe a contrastare le tesi capitali o ad accoglierle eangiandole profondamente. Il diritto naturale gli offerse non soluzioni ma problemi, e di questi anche se alcuni gli offerse ben determinati, altri, e piú gravi, suseito solamente nel suo spirito: problemi dunque o non risoluti o neppure veduti, che il Vico si propose e in parte risolse.

Gli aspetti e le tendenze del diritto naturale erano molteplici, e conviene preliminarmente distinguerli ed enumerarli. In primo luogo, in quella scuola, presa nel suo complesso e nei suoi tratti cssenziali, si esprimeva il progresso sociale, onde l'Europa, uscendo dal feudalesimo e dalle guerre di religione, si dava una nuova coscienza, spiccatamente borghese e laica : si ricordi che la formazione di essa fu quasi contemporanea alla nascita dell'anticlericale e borghese istituto della «massoneria . "Naturale» voleva dire, tra l'altro, «non soprannaturale»; e, quindi, ostilità o indifferenza di fronte al soprannaturale e alle istituzioni che lo rappresentavano e ai conflitti sociali che ingenerava. Non a caso il Grozio fu arminiano; il Pufendorf ebbe liti con teologi; il Tomasio è rammentato tra i promotori della liberti di coscienza. Le proteste di reverenza verso la religione e verso la chiesa, che con molta 
abbondanza quei pubblicisti solevano inserire nei loro scritti (i quali ne sono come soffusi da un velo di pietà), erano cautele da politici, che procurano di minare il nemico senza lasciarsi scorgere, di ferire coprendosi. Cautela lodata, per es., nel Grozio da uno dei seguaci della scuola (l'autore della Pauco plenior iuris naturalis historia, 1719), che celebra il maestro come "instrumentum divina providentice », quasi Messia venuto a redimere il * lumen naturale » dalla servitú al «supernaturale », e fornito pereio di tutta la forza e di tutta l'abilita occorrenti; talché, esperto delle persecuzioni scolastiche, «caute versabatur.... ne maius bilem adversus prudentiam naturalem et rationalem ex latebris productam tam minis irritaret $\gg$, e procedendo a separare le leggi umane dalle divine, non prendeva di fronte la scuola teologica con l'attaccarne gli errori fondamentali, anzi perfino la lodava nei prolegomeni dell'opera sua. "Naturale » significava altresi ciò che è comune agli individui delle varie nazioni e stati; onde, sotto l'aspetto pratico, forniva un ottimo motto d'ordine per riunire in certi desiderî, speranze e lotte comuni la borghesia dei varî paesi. I trattati del diritto naturale furono, nel secolo decimosettimo e nel seguente, per la borghesia, quel che il Manifesto dei comunisti e il grido: "Proletarî di tutto il mondo, unitevi », tentarono di essere per la elasse operaia nel decimonono.

In quanto quella scuola e quella pubblicistica erano manifestazione di un moto pratico, l'interesse filosofico vi aveva parte subordinata e ufficio sussidiario. Per questa ragione, in secondo luogo, le trattazioni del diritto naturale, filosoficamente considerate, non si levano di solito sopra un chiaro e popolare empirismo. I prineipî, sui quali si appoggiano, non sono approfonditi e assai spesso neppure estrinsecamente unificati; i concetti, che adoperano, sono piuttosto rappresentazioni generali; la forma della tratta- 
zione è solo apparentemente sistematica. Qualcuno di quegli scrittori procurava di collegare le sue dottrine giusnaturalistiche con la filosofia platonica, stoica o cartesiana, risaliva ad assiomi logici e metafisici, si giovava della deduzione e clel metodo matematico. Ma tutto codesto era accostamento e non fusione, adornamento e non ravvivamento; e, tutt'al piú, valeva come prova di diligenza e di serietà d'intenzioni.

La filosofia, per altro, implicita piú o meno nei trattatisti del diritto naturale ed osplicita nei filosofi che prosero a elaborarlo speculativamente, si accordava con lo spirito del tempo, del quale ci sono noti i caratteri generali. Cosicché terzo aspetto del giusnaturalismo fu, in etica, o l'utilitarismo, ora piú o meno larvato ora apertamente dichiarato, e a volta a volta ragionato con filosofia piuttosto matematizzante o piuttosto sensistica, di tendenze materialistiche o di tendenze razionalistiche; ovvero (che è quasi il medesimo) un astratto e intellettualistico moralismo, che minacciava di procipitare a ogni istante nell'utilitarismo. Dal quale intellettualismo e utilitarismo, combinati con l'impronta pratica e rivoluzionaria di quel moto spirituale, che era rivolto piuttosto a un semplicistico diritto da far trionfare che non a riconoscere quello realmente svoltosi nella storia e riceo di tante forme e vicende, derivava il quarto carattere di esso, cioè la mancanza di senso storico, l'antistoricismo della scuola, la quale stabiliva l'astratto ideale di una natura umana fuori della storia umana o non fusa e vivente in questa.

Infine, borghese, anticlericale, utilitario o materialistico com'era, il giusnaturalismo aveva un quinto e importante carattere, l'avversione alla trascendenza e la tendenza a una concezione immanentistica dell'uomo e della società. Carattere poco esplicato e poco ragionato dottrinalmente, ma non pertanto facilmente riconoscibile nel complesso dei concetti di quella scuola. 
Ora, l'ispirazione del Vico era genuinamente ed esclusivamente teoretica, punto pratica o riformistica; altamente speculativo il suo metodo, e disdegnoso dell'empirismo; idealistico, e perciò antimaterialistico e antiutilitaristico, il suo spirito; la sua gnoseologia anelante al concreto, al certo, e però storicizzante. Per conseguenza, la sua dottrina della ragion pratica, pure prendendo le mosse dal giusnaturalismo, doveva uscire diversa, anzi contraria a questo, in tutti i primi quattro caratteri da noi enunciati. E se in qualcosa coincideva (non nella via per pervenirvi, ma nel risultamento), era appunto dove meno l'autore avrebbe voluto: nel carattere immanentistico e areligioso.

Ma poiché il nostro proprio tema non è già la critica e modificazione che il diritto naturale ebbe nel pensiero del Vico, sí bene questo pensiero stesso, sarà opportuno, ripigliando il filo della esposizione, seguire ordine alquanto diverso da quello tenuto nel ricapitolare $\mathbf{i}$ varî caratteri del giusnaturalismo, e cominciare dal vedere l'opposizione del Vico all'utilitarismo dichiarato o larvato di quella scuola, e la dottrina che egli svolse sul principio dell'etica.

I due principali rappresentanti dell' utilitarismo nel secolo decimosettimo, che il Vico ha sempre innanzi agli occhi, sono l'Hobbes e lo Spinoza; ma ricorda insieme con essi il Locke e il Bayle e, del secolo precedente, il Machiavelli e, risalendo all'antichità, gli stoici col loro concetto del fato, gli epicurei con quello del caso, Carneade col suo scetticismo, e perfino l'inconsapevole dottrina che è contenuta nel motto « Vee victis », attribuito al Brenno o capo dei Galli invasori di Roma. Dell' Hobbes ammirava lo sforzo magnanimo nel cercare di accrescere la filosofia di una teoria che le era mancata nei bei tempi della Grecia, cioè della teoria dell'uomo considerato in tutta la società del genere umano; ma diceva infelice l'evento, fallito il ten- 
tativo, che (come anche quello del Locke) nel fatto risultava assai prossimo all'cpicureo. L'Hobbes non si era accorto che egli non si sarebbe potuto neppure proporre il suo problema del diritto naturale dell'umanita, se il motivo non gliene fosse stato fornito per l'appunto dalla religione cristiana, la quale comanda verso tutto il genere umano, nonché la giustizia, la carità. Agli stoici invece, al loro fato e al loro determinismo onde furono incapaci a ragionare adeguatamente di repubblica e di leggi, a codesti « spinosisti dell'antichita », si collegava idealmente lo Spinoza, del cui utilitarismo, diverso di spiriti tanto dal lockiano quanto dall' hobbesiano (perché lo Spinoza « mente, non sensu de veris rerum diiudicat $»$ ), non isfuggiva al Vico la singolarità. Ma, per singolare che debba dirsi, esso costrinse lo Spinoza a ragionare di repubblica in modo poco elevato, «come di una società che sia di mercadanti ». Quelle dottrine utilitarie, calunniose dell'umana natura, parvero al Vico proprie di uomini disperati, che per la loro vilta non ebbero mai parte nello stato, o per la loro superbia si stimarono tenuti bassi e non promossi agli onori dei quali per la loro boria si credevano degni; e annoverò tra costoro il povero Spinoza, il quale, non avendo, perché ebreo, niuna repubblica, mosso da livore, si sarebbe dato a escogitare una metafisica * da rovinare tutte le repubbliche del mondo *. Severo è il suo giudizio sulle condizioni dell'etica ai suoi tempi, che era quale poteva essere sulla base di una metafisica meccanica e materialistica, senza lume di finalita. Cartesio fu affatto sterile in quel campo, perché le poche cose che sparsamente ne lasciò scritte non compongono dottrina e il suo trattato delle Passioni serve piuttosto alla medicina che alla morale; similmente sterili il Malebranche o il Nicole, e i Pensieri del Pascal, solitaria eccezione, sono «pur lumi sparsi ». Degli italiani, il Pallavicino offri appena un abbozzo di etica nel 
suo trattato Del bene, c il Muratori, nella sua Filosofia morale, fece prova assai infelice.

L' utilità non è principio esplicativo della moralità, perché proviene dalla parte corporale dell'uomo e, per tale provenienza, è cangevole, laddove la moralita, l'honestas, è eterna.-Derivare la moralità dall'utilità è scambiare l'occasione con la causa, fermarsi alla superficie e non spiegare per nulla i fatti. Nessuno dei varî modi nei quali il principio utilitario viene atteggiato dai filosofi, la frode o impostura, la forza, il bisogno, rende conto delle differenziazioni, cioè dell'organismo sociale. Quale frode poteva mai sedurre e trarre in inganno $\mathrm{i}$ supposti primi semplici e parchi posseditori di campi, i quali vivevano affatto contenti della sorte loro? Quale forza, se i ricchi, i pretesi usurpatori, erano pochi, e i poveri, i derubati, molti? Codeste spiegazioni sono giochetti, indegni del grave problema. Quei forti, quei potenti erano, in realta, potenti d'altro che di sola forza; tanto che si facevano protettori dei deboli e oppugnatori delle tendenze distruttive e antisociali: la loro legge era, sí, di forza, ma « a natura prostantiori dictata, cosa che ben era lecito ignorare al barbaro Brenno, ma non a uomini filosofi. La forza creatrice e organizzatrice delle prime repubbliche fu tutta umanita generosa, alla quale si debbono richiamare sempre gli Stati, quantunque acquistati con l'impostura e con la forza, perché reggano e si conservino; conformemente al detto del Machiavelli di richiamarli alle origini, ma con l'intesa che le origini profonde si trovano nella clemenza e nella giustizia. Gli uomini sono tenuti insieme da qualcosa di piú saldo dell'utilità. Società d'uomini non può incominciare e durare senza fede scambievole; senza che altri riposino sopra le altrui promesse e si acquetino alle altrui asseverazioni di futti occulti. Si può forse ottenere questa fcde col rigore delle leggi penali 
contro la menzogna? Ma le leggi sono prodotto della societa, e, perché sorga societa, è necessaria quella fede scambievole. Si dira, come dice il Locke, che si tratta di un processo psicologico, pel quale gli uomini via via si avvezzano a credere quando altri loro dica e prometta di narrare la verita? Ma, in questo easo, quegli uomini gia intendono l'idea di un vero, che basti rivelare per obbligare altrui a doverlo credere senza niun documento umano; e il principio psicologico dell'abitudine è oltrepassato.

La causa vera della società umana non è, dunque, l'utilita, la quale favorisce soltanto, come occasione, l'azione della causa, e fa si che gli uomini, per natura sociale deboli e indigenti, e divisi dal vizio di origine, si traggano a celebrare la loro natura sociale, « rebus ipsis dictantibus , secondo la formola pomponiana, che il Vico ripete con predilezione. Cose, fatti, circostanze mutano nella moralità che non muta; e di qui l'illusione degli utilitaristi, che guardano dall'esterno e si tengono alle apparenze e vedono il mutamento e non la costanza. L'omicidio è vietato; ma l'approvazione che si dà a colui il quale, minacciato nella vita, non potendo altrimenti salvarsi, uccide l'ingiusto aggressore, non importa mutevolezza del criterio morale circa l'omicidio, perché, in quelle particolari circostanze, non si tratta, in realta, di omicidio, ma di pena capitale che l'ingiustamente aggredito, trovandosi in solitudine, infligge quasi per tacita delegazione sociale. Il furto $\partial$ vietato; ma colui che, per tenersi in vita, prende altrui un pane, non viola la moralita, perché esercita un diritto fondato sull'equobono.

La sola filosofia che porti con sé una vera etica sembra al Vico la platonica, risalente a un principio metafisico, l'idea eterna che educe da sé e crea la materia; laddove l'etica aristotelica è fondata sopra una metafisica che conduce a un principio fisico, alla materia, dalla quale 
si educono le forme particolari facendo di Dio un vasellaio che lavori le cose fuori di sé. L'etica dei giureconsulti romani abbonda, senza dubbio, di splendidi aforismi, ma non è altro che una semplice arte di equità, insegnata con innumerabili minuti precetti di giusto naturale, che quelli indagavano dentro le ragioni delle leggi e la volontà del legislatore; epperò non può considerarsi come filosofia morale, dove fa d' uopo procedere da pochissime verità eterne, stabilite in metafisica da una giustizia ideale. Per ragioni analoghe il Vico non poteva appagarsi del Grozio e degli altri ginsnaturalisti; circa i quali nota in genere cosa verissima, cioè che i loro grossi volumi recano, sí, titoli magnifici, ma poi non contengono nulla piú di ciò che è volgarmente risaputo. Se si pesano i principî del Grozio con la bilancia esatta della critica, risultano tutti piuttosto probabili e rerisimili che necessarî e invitti. Nella questione dell'utilità il Grozio non coglie il punto giusto, non distinguendo l'occasione dalla causa; né * inchioda », ossia non definisce, l'antichissima disputa se il diritto sia in natura o solo nelle opinioni degli uomini, nella quale filosofi e teologi ancora contendono con lo scettico Carneade e con Epicuro; propone l'ipotesi degli uomini primitivi che siano « semplicioni », ma si dimentica affatto di ragionarla. E poiché quei suoi «semplicioni », accortisi dei danni della solitudine bestiale, vengono alla vita comune, e questa determinazione è loro dettata dall'utilità, il Grozio scivola anche lui, senza avvedersene, nell'utilitarismo e nell'epicureismo.

Ma il Vico, invece, alla domanda se il diritto sia per natura o per convenzione risponde con la solenne \& dignità *: * Le cose fuori del loro stato naturale né vi si adagiano né vi durano ». Alla domanda donde nasca la società risponde richiamando il senso umano, la coscienza, il bisogno che ha l'uomo di salvarsi dal nemico interno che gli 
rode il petto. L'origine è certamente nel timore, ma nel timore di sé stesso, non della violenza altrui; è nel rimorso che punge, nel pudore che tingendo di rosso il volto dei primi romini fa risplendere per la prima volta la moralità sulla terra. Dal pudore nascono tutte le virtú, l'onore, la frugalità, la probità, la fede nelle promesse, la verita nelle parole, l'astensione dall'altrui, la pudicizia. Celebrando la società, l'uomo celebra la natura umana.

Il pudore o coscienza morale, tradotto nella corrispondente scienza empirica, dà il senso comune degli uomini d'intorno alle umane necessita o utilita, che è la fonte del diritto naturale delie genti. Questo senso comune (dice il Vico) è un giudizio senza alcuna riflessione, comunemente sentito da tutto un ordine, da tutto un popolo, da tutta una nazione e da tutto il genere umano. Giudizio senza riflessione non è veramente giudizio, dal quale la riflessione è inseparabile; non è giudizio anche perché sentito e non pensato. Ma non è neppure quello che poi si disse « sentimento», termine vago, jgnoto al Vico non meno che alla filosofia tradizionale. E piuttosto un atteggiamento pratico che, simile a un di presso negl' individui viventi in condizioni simili, produce i simili costumi dei varî gruppi sociali, da quelli di una classe particolare a quelli dell'intera umanità. Atteggiamento affatto spontaneo (e, anche per questo definito privo di riflessione), onde i costumi si generano dall'interno e non dall'esterno, e sono simili senza che siano copiati gli uni dagli altri ( senza prendere esempio l'una nazione dall'altra »). Attraverso quel senso comune la coscienza morale s'incorpora in compatti e resistenti istituti; ed esso accerta l'umano arbitrio, che è di sua natura incertissimo. 


$$
\text { - }
$$




\section{VII}

\section{Morale e religione}

Ma il timore interno, il pudore, la coscienza morale è svegliata negli uomini dalla religione: il timore è timore di Dio, il pudore è vergogna innanzi a lui. Gli uomini primitivi errano per la terra solitarî, selvaggi, feroci, senza lingue articolate, senza concubiti certi, in preda alle loro disordinate violentissime passioni; piuttosto che uomini, «bestioni ». Chi li frenera? Donde verra il soccorso che. loro inpedisca di distruggersi a vicenda? Non possono indirizzarli uomini sapienti, che non si sa donde o come s'introdurrebbero in mezzo a loro; non puo salvarli l'intervento di Dio: Dio si è ritirato nel suo popolo eletto e non ha nessun commercio con la restante umanità, con l'umanita gentilesca. Ma quei « bestioni » son pur uomini: Dio, nell'abbandonarli, ha lasciato nel fondo del loro cuore una favilla dell'esser suo. Ecco: il cielo fulnina, i bestioni stupiscono, si fermano, temono; si accende in loro la confusa idea di qualcosa che li supera, di una divinita. Ed essi pensano, o piuttosto immaginano, un primo Dio, un Cielo o un Giove fulminante; e a quel Dio si rivolgono per placarlo o per invocarlo a soccorso. Ma per placarlo e averlo soccorritore debbono conformare la propria vita a questo intento: umiliarsi alla divinita, domare l'orgoglio e la fie- 
rezza, astenersi da certi atti, compierne altri. Dal pensiero della divinita riceve forza dunque il conato ossia la libertà, che è propria della volontà umana, di tenere in freno i moti impressi alla mente dal corpo per acquetarli o per dare loro altra direzione. E con questi atti di dominio sopra sé stesso, con la libertà, è nata insieme la mo-. ralita: il timore di $\mathrm{Dio}$ ha posto il fondamento alla vita umana. La terra si copre di are; le grotte dei suoi monti, dove il maschio trascina ora la femmina, vergognoso dei concubiti innanzi al volto del Cielo o di Dio, assistono ai primi riti nuziali, proteggono le prime famiglie; il grembo della terra si apre ad accogliere il pio deposito dei morti corpi. Le prime e fondamentali istituzioni etiche - culto religioso, matrimonî, sepolture - sono sorte.

Questa potenza etica- e sociale dell'idea di Dio si riafferma nel corso della storia posteriore; perché, quando i popoli sono infieriti con le armi, e nessun potere hanno piú sopra di loro le umane leggi, l'unico mezzo di ridurli è la religione. Si riafferma nello svolgimento individuale della vita umana: ai fanciulli, infatti, non si può altrimenti insegnare la pietà che col timore di qualche divinità; e, nella disperazione di tutti i soccorsi della natura, l' uomo desidera un essere superiore che lo salvi, e questo essere è Dio. Tutte le nazioni credono in una divinita provvidente: popoli che vivano in società senza alcuna. coscienza di Dio, per es., in alcuni luoghi del Brasile, in Cafra, nelle Antille, sono novelle di viaggiatori, che procurano smaltimento ai loro libri con mostruosi ragguagli.

Se è cosi (e cosí è certamente), nessuna dottrina è piú stolta di quella che pretende concepire morale e civiltà senza religione. Come delle cose fisiche non si può avere certa scienza senza la guida delle verità astratte fornite dalle matematiche, delle cose morali non si può senza la scorta delle verità astratte metafisiche, e perciò 
senza l'idea di Dio. Quando si spegne o si oscura la coscienza religiosa, insieme si spegne e si oscura il concetto di societa e di stato. Ebrei, cristiani, gentili e maomettani ebbero quel coneetto, perché tutti credettero in qualche divinità, sia come mente infinita libera, sia come piú dèi composti di mente e di corpo, sia come un unico Dio, mente infinita libera in corpo infinito. Ma non lo ebbero gli epicurei, che attribuivano a Dio il solo corpo e col corpo il easo; né gli stoici, che lo fecero soggetto al fato. E ottimamente Cicerone diceva ad Attico, epicureo, di non potere istituire con lui ragionamento intorno alle leggi, se prima non gli concedesse che vi sia provvidenza divina. L'Hobbes, che rinnovava l'epicureismo, e lo Spinoza, rinnovatore dello stoicismo, si è visto che non intesero nulla di quel che siano società e stato. Tra gli empî uomini primitivi, brutti, irsuti, squallidi, rabbuffati, dovrebbero andarsi a disperdere quei dotti dalla \& sfumata letteratura », e a capo di essi Pietro Bayle, che sostengono che senza religione possa vivere, e viva di fatto, umana società.

La manchevolezza nell'idea di Dio è altresí il principale argomento della critica che il Vico muove a due di coloro che egli altamente onorava come "príncipi» del diritto naturale, al Grozio e al Pufendorf. Né l'uno né l'altro (egli dice) statuisce per primo e proprio principio la provvidenza divina. Il Grozio non gia che propriamente la neghi, ma, * per lo stesso grande affetto che porta alla verità », per meglio assodare la necessità razionale dell' umana societa, ne vuol prescindere, e professa che il suo sistema regga, tolta anche ogni cognizione di Dio; onde il Vico lo taccia di socinianismo, perché pone la naturale innocenza in una semplicita di natura umana. Peggio il Pufendorf, il quale addirittura sembra sconoscere la provvidenza e comincia con un'ipotesi scandalosa ed epicurea, suppo- 
nendo l'uomo gettato in questo mondo senza niun aiuto e cura di Dio (senza neppure quella scintilla chiusa in petto, che si dilaterà in fiamma morale); della qual cosa essendo stato ripreso (dallo Schwartz), cerco di giustificarsi con una particolare dissertazione (l'Apologia del 1686), ma non giunse a scorgere il principio vero che solo rende possibile spiegare la società.

Ora perché mai, essendoci note tutte codeste energiche affermazioni e polemiche del Vico sulla condizionalità religiosa della morale, abbiamo asserito che il solo punto in cui egli si trovi veramente d'accordo col Grozio, col $\mathrm{Pu}$ fendorf, e in genere con la scuola del diritto naturale, è la concezione affatto immanente dell'etica? - Perché, se ben si osservi, il Vico non si oppone al metodo tenuto dai giusnaturalisti; ché anzi anch'egli costruisce la sua scienza della società umana prescindendo, come il Grozio, da ogni idea di Dio, e, come il Pufendorf, ponendo l'uomo senza aiuto e cura di Dio, cioè prescindendo dalla religione rivelata e dal Dio di essa. Come per quei due, materia della sua indagine è il diritto naturale e non il soprannaturale, il diritto delle genti e non quello del popolo eletto, il diritto che sorge spontaneo nelle caverne e non quello che scende giú dal Sinai. L'opposizione del Vico (da lui esposta con la consueta confusione e oscurita) si aggira non sopra codeste affermazioni, ma sul concetto stesso di religione. La religione, insomma, della quale egli parla, non è la medesima di cui parlavano, o non parlavano, il Grozio e il Pufendorf.

Religione, come già sappiamo, vale per il Vico non gia rivelazione ma concezione della realtà; o che si affermi, come nei tempi della mente tutta spiegata, in forma di metafisica intelligibile, e mova dal pensiero di Dio per schiarire la logica nei suoi raziocinî e discendere a purgare il cuore dell'uomo con la morale; o che si affacci, 
come nei primordî dell'umanitả, in forma di metafisica poetica. Dalla religione rivelata, quando si ricerchi il fondamento della morale, si pù ben prescindere; ma in qual modo si potrebbe da quella religione naturale, che è tutt'una cosa con la coscienza della verità? Plutarco, descrivendo lo primitive religioni spaventevoli, pone in problema se, invece di venerare cosi empiamente gli dèi, non sarebbe stato meglio che non fosse esistita religione alcuna; ma egli dimentica che da quelle fiere superstizioni si svolsero luminose civiltà e sull'ateismo non crebbe mai nulla. Senza una religione, mite o feroce, ragionata o immaginosa, che dia l'idea piú o meno determinata e piú o meno elevata di qualcosa che superi gl'individui e in cui gli individui tutti si raccolgano, mancherebbe alla volonta morale l'oggetto del suo volere.

E a questo punto si chiarisce quello che abbiamo distinto come il sccondo significato, pratico o etico, della parola "religione * nel Vico. Nel qual significato egli rivendica e giustifica il detto degli empî che * il timore fece gli dèi »; o, anche, addita la radice della religione nel desiderio che gli uomini hanno di vivere eternamente, mossi da un senso comune d'immortulità nascosto nel fondo della loro mente. La religione è, in questo secondo significato, un fatto pratico ossia la moralita stessa, come nel primo era la verità stessa.

Intesa dunque la religione dal Vico o (nel primo significato) come condizione o (nel secondo) come sinonimo della moralita, è chiaro che, col censurare il Grozio e il Pufendorf per la loro trascuranza di questo importantissimo concetto, egli non faceva altro in sostanza che ribadire la critica all'insipido moralismo e al larvato utilitarismo di quei due pensatori. E pel medesimo fine ebbe anche altre volte ricorso all'efficace strumento del concetto di religione. Perché se alla filosofia attribuí talora l'ufficio di 
giovare il genere umano sollevando e reggendo l' uomo caduto, tal'altra giudicò che essa sia piuttosto adatta a ragionare, e che le massime ragionate dai filosofi intorno alla morale servano solamente all'eloquenza per accendere i sensi a compiere i doveri della virtú, laddove solo la religione è efficace a far virtuosamente operare. Nella scienza empirica, poi, che corrisponde a questa parte della filosofia dello spirito, il Vico, mutate in due epoche storiche la religione (o metafisica poetica) e la filosofia, fatto della prima il carattere dell'epoca barbarica e della seconda quello dell'epoca civile, è ovvio che dovesse sostenere, come sostenne, che sola fondatrice di ogni civilta e della stessa filosofia è la religione, e rigettare il detto (che egli, non senza ritoccarlo, attribuiva a Polibio) che, se ci fossero al mondo filosoti, non farebbero uopo religioni. Come potrebbero sorgere filosofi (egli obietta), se prima non sorgano le repubbliche ossia le civiltà? e come le repubbliche potrebbero sorgere, senza l'opera delle religioni? Quel detto si deve dunque invertire: senza religione, nessuna filosofia. Fu la religione, fu la provvidenza divina, che addimesticò i figliuoli dei Polifemi e via via li ridusse all'umanità degli Aristidi e dei Socrati, dei Lelî e degli Scipioni Africani.

Anche il concetto dello stato ferino, che nei libri dei giusnaturalisti serviva da ipotesi e da espediente didascalico, sia per isvolgere la trattazione indipendentemente dalla teologia mistica senza sollevare troppi scandali, sia per insinuare le loro teorie utilitaristiche, nel Vico ricompare con nuovo ufficio e nuovo contenuto. Cattolico di pure intenzioni, avendo dato pace al suo animo col separare la religione rivelata da quella umana, egli è in grado di assumere lo stato ferino come vera e propria realtà. Verità ideale, in quanto rappresenta nella dialettica della coscienza pratica un momento necessario per la genesi della noralita (il momento premorale); realtà storica ed empi- 
vica, come approssimativa condizione di fatto in quei periodi di anarchia e fermentazione che precedono il sorgere della civiltà o seguono alle crisi di queste. I giusnaturalisti facevano ossequio, ora piú ora meno, alla dottrina tradizionale della chiesa, cioè che l'umanità gentilesca, nella dispersione seguita alla confusione babelica, avesse portato seco un residuo di religione rivelata, un vago ricordo del vero Dio, donde l'origine della vita sociale e degli dèi falsi e bugiardi, barlume del Dio vero; e per questa ragione lo «stato ferino " veniva proposto nel loro sistema come astratto e irreale. Il Vico eseguiva sul serio la distinzione tra ebrei e gentili, e concepiva lo stato ferino come privo di ogni aiuto che provenisse dall'anteriore rivelazione: uno stato nel quale l'nomo era, per cosí dire, da solo a solo con le proprie sconvolte e turbolente passioni.

Stato di fatto senza moralità, ma (diversamente che nell'ipotesi utilitaria) tutto pregno di esigenze morali, e dal quale si esce col farsi esplicito di questo implicito. Ma si esce naturalmente e non gia per effetto della grazia divina: la vera grazia divina è la stessa natura umana, a cui partecipano i gentili al pari degli ebrei, tutti irraggiati nel volto da un lume divino.

L'uomo ha libero arbitrio, ma debole, di fare delle passioni virtú; e nel suo travaglio verso la virtú è aiutato in modo naturale da Dio con la provvidenza. Di certo, il Vico non intende disconoscere l'efficacia altresí della diretta e personale grazia divina; ma, col suo solito metodo, la separa dalla provvidenza naturale, che sola gl'inporta e sola considera. A lui piacque sempre, per quel che concerneva le controversie sulla grazia, di tenersi lontano dai due estremi, tipicamente rappresentati, secondo lui, dal pelagianismo e dal calvinismo; e tin da giovane, studiando le opere del Ricardo (il gesuita Stefano Deschanps), teologo della Sorbona, ne accettò la dimostrazione circa l'ec- 
cellenza della dottrina agostiniana, appunto perché media tra quegli estremi. Siffatta temperata dottrina gli sembrava propria (diceva) per meditare un principio di diritto naturale delle genti, che spiegasse l'origine del diritto romano e di ogni altro gentilesco, e per tenersi nel tempo stesso in accordo con la religione cattolica. Era disposto a concedere che vi sia una nazione privilegiata, l'ebrea; e che l'uomo cristiano, nella lotta contro le passioni, sia piú forte del non cristiano, perché, dove non giunge la grazia naturale, può essere soccorso dalla soprannaturale. Ma, infine, il miracolo è miracolo, e la Scienza nuova non è scienza di miracoli.

Che tale non sia, è confermato dalla critica del Vico al terzo dei tre * principi » del diritto naturale, a Giovanni Selden, celebre ai suoi tempi quanto dimenticato poi, autore del De iure naturali et gentium iuxta disciplinam hebraorum (1640). Diversamente dal Grozio (e avversario di lui anche in altre questioni), il Selden non negava anzi sublimava l'eficacia della religione, né concepiva possibilita alcuna di vita morale e civile per il genere umano, fuori della rivelazione. La quale, fatta da Dio al popolo ebreo, da questo sarebbe passata ai gentili per molteplici vie di trasmissione: Pitagora, per es., avrebbe avuto per maestro Ezechiele; Aristotele, al tempo della spedizione di Alessandro in Asia, si sarebbe stretto in amicizia con Simone il giusto; a Numa Pompilio sarebbe giunta qualche notizia della Bibbia e dei profeti. C'era di che soddisfare ogni animo di credente, che si ritraesse timoroso dai libri degli altri giusnaturalisti avvertendone le tendenze eterodosse. Ma il Vico non vuol sapere di codesto sistema ultrareligioso. Se il Grozio prescindeva dalla provvidenza e il Pufendorf la sconosceva, il Selden aveva il torto (egli dice) di supporla, di farne cioè un deus ex machina, senza spiegarla con l' intrinseca natura della mente umana. Contrario 
alla filosofia, quel sistema non era meno contrario alla storia sacra, la quale anche per gli ebrei ammette in certo modo un diritto non rivelato ma naturale, e solamente perché essi ne persero coscienza nel tempo della schiavitú d'Fgitto, fa intervenire l'opera diretta di Dio con la legge data a Mosé; - e non era conforme, nell'asserita trasfusione di cognizioni e leggi dagli cbrei nei gentili, a quel che dice Flavio Giuseppe degli ebrei, sempre restii a qualsiasi contatto con popoli stranieri, e a quel che il Vico supponeva fosse detto ancle a questo proposito da Lattanzio, come in genere era privo di qualsiasi piú elementare sussidio di documenti. Cosicché lá conclusione del Vico è sempre la medesima: gli ebrei si giovarono altresí di un aiuto straordinario del vero Dio, ma le restanti nazioni s'incivilirono per opera dei soli lumi ordinarî della provvidenza.

Se poi il Vico interpetrasse esattamente il Grozio e il Pufendorf ed esattamente ne riferisse le parole, è quesito per noi di lieve peso, perché non tanto c'importa il modo nel quale il Vico espose e giudicò gli altri filosofi, quanto le idee che egli sostenne pur attraverso i suoi fraintendimenti storici, che, a dir vero, non sono pochi. Tuttavia, sarà bene indicare di volo, circa le difficoltà che possono incontrarsi su questo punto, la soluzione che a noi sembra plausibile. Senza dubbio, chi, dopo aver letto le censure del Vico, apra il De iure belli et pacis e vi trovi che il Grozio include espressamente fra i suoi tre principî fondamentali, accanto alla ragione e alla socialità, la volonti divina, e che quel suo prescindere da Dio suona poco piú di una semplice frase enfatica a siguificare la forza della socialità e della ragione (le quali avrebbero efficacia « etiamsi daremus non esse Deum $\gg$ che Dio non si curi delle cose umane, quod sine summo scelere dari nequit >); - chi apra il Pufendorf e vi legga il piú solenne rifiuto dell'ipotesi groziana, cmpia ed assurda, e la dichiarazione che le 
Ieggi naturali resterebbero sospese in aria, prive di forza, senza la volontà di un Dio legislatore; - può essere tratto a tacciar il Vico di poca diligenza o di strana puntigliositá ortodossa nella critica che muove a questi suoi predecessori. Ma il Vico, in verită, non sapeva che cosa farsi di un Dio messo accanto alle altre fonti della moralità, o messovi disopra come una superflua fonte della fonte; egli, che cercava Dio nel cuore dell' uomo, sentiva e scorgeva l'abisso che lo separava da coloro che non l'avevano piú nel cuore e appena, per abito o per prudenza, lo serbavano nelle parole. Piú sottilmente si potrebbe domandare perché mai, se il Vico era d'accordo coi giusnaturalisti nel prescindere dalla rivelazione, e se egli, anziché rigettare, approfondiva la loro superficiale dottrina immanentistica, si atteggiasse a loro risoluto avversario e facesse la voce grossa e insistesse presso prelati e pontefici nell'attribuirsi il vanto di aver esso pel primo formato un sistema del diritto naturale, diverso da quello dei tre autori protestanti e adatto alla chiesa romana. L'ipotesi che operasse cosí per politica cautela la proporremmo, se, invece di lui, avessimo innanzi, per es., un appassionato e magnanimo ma furbo frate, un Tommaso Campanella; ma la candida personalità del Vico la esclude affatto, e solo si può concedere che, poco chiaro com'era sempre nelle sue idee, questa volta si adagiasse alquanto nella poca chiarezza e, trasportato dalla sua calda fede, alimentasse le sue illusioni, fino a idoleggiarsi dentro di sé con la veste di defensor ecclesice nell'atto stesso che soppiantava la religione della chiesa con quella dell' umanità. 


\section{VIII}

\section{Morale e Diritto}

Dopo che tanto lume di originalita è rifulso ai nostri occhi, non ci riesce possibile fermare lo sguardo su quelle dottrine c classificazioni etiche che il Vico attinse alla filosofia tradizionale e mise soprattutto nel primo libro del Diritto universale; quantunque esse per l'appunto riescano assai care a non pochi lettori e siano divenute quasi popolari per le conţinue citazioni che ne sono state fatte. Che Dio sia * posse, nosse, velle infinitum s e l'uomo " posse, nosse, velle finitum quod tendit ad infinitum ; - che la re. pubblica o Stato sia immagine di Dio, e poiché « omnia infra se, nil superius habet », debba « uni Deo, prceterea nemini reddere rationem », e come la liberta di Dio inerisce alla sua eterna ragione, cosí lo Stato liberamente ubbidisea alli legge da esso stesso stabilita; - che la giustizia * utilitates dirigat et excequet », comandando nella costruzione delle repubbliche quasi architetta alle due giustizie particolari commutativa e distributiva, due fabre divine ehe misurano le utilita con le due misure eterne, aritmetica e greometrica, sicché "quorl est aquum cum metiris, idem est iustum quum eligis "; - queste e altrettali sentenze, oltre che poco originali, ci sembrano o fallaci o vuote, sebbene possano adornarsi dei nomi ora di Aristotele ora del 
Campanella ora di altri filosofi antichi o del Rinascimento. Se, per dirne una, la giustizia consistesse in misurazioni, una filosofia della giustizia non farebbe d'uopo, dovendo bastare le scienze del calcolo e della misura. Il Vico medesimo, in un punto, svela involontariamente e ingenuamente il circolo vizioso di quella metafora scambiata per concetto, dicendo che gli uomini debbono * comunicare tra loro egualmente le utilita, solamente serbata una giusta differenza ove si tratta di meriti, e questa stessa per serbar l'egualità».

Meglio clie queste viete formole, gioverebbe andare raccogliendo le frequenti c acute osservazioni di psicologia morale che si trovano sparse nei suoi scritti, espresse in istile lapidario; o ricordare la sua poco nota teoria sul riso, che egli faceva nascere dall'aspettazione delusa e dalla debolezza della mente e che perciò negava cosi all'animale come all' uomo perfetto, considerando l'uomo che ride come un satiro o fauno, medio tra la 'bestia e l'uomo. - Ma, astenendoci da questa raccolta che è estranea al nostro intento, noteremo piuttosto che, anche nelle sopramenzionate comuni distinzioni e classificazioni, il Vico ha un qualche merito; cioè, per l'appunto, dí riconoscere, nel tempo stesso che le propone, il necessario confondersi e identificarsi di tutte o di molte tra quelle distinzioni. Cosí, distinte le due giustizie, le tre virtú e i tre diritti, finisce col dichiarare che codeste dualità, trinità e molteplicità fanno tutt'uno.

E tutt'uno fanno per lui anche giustizia e virtú, giacché quella forza del vero o ragione umana, che è virtú in quanto pugna con la cupidità, la medesima è giustizia in quanto dirige e agguaglia le utilità. Il che vuol dire che il Vico non distinse, almeno nella esposizione sistematica del Diritto universale, il diritto dalla morale: distinzione che, in verita, ebbe poco rilievo nel giusnaturalismo e ap- 
pena è adombrata (per es., nel Grozio) come quella di un piú e di un meno di moralità. Affatto moralistica, e dedotta dal concetto etico di rimorso, è altresi la dottrina vichiana della pena, la quale, infitta dalle leggi, non sarebbe per lui altro che il supplemento sociale alla coscienza individuale nel caso in cui non si purga da sé per mezzo del rimorso e della pena interiore.

Ma il problema del rapporto tra diritto e morale quanto piú, presso il Vico, è assente dalla formolazione teorica e dalla trattazione sistematica, tanto piú è presente nelle osservazioni particolari e circola, si può dire, in tutta la Scienza nuova. Né poteva essere altrimenti, atteso che quel rapporto rimanda alla distinzione tra la volontà morale e le forme di volonta inferiori o anteriori; e noi sappiamo che il Vico era da tutte le sue tendenze portato a profondarsi clentro la regione inferiore e oscura dello spirito, cosí conoscitiva come pratica, cosí della fantasia come dell'arbitrio e della passione.

Delle passioni avvertí sempre la somma importanza; c se non poteva approvare il darsi loro in preda, e se giudicò sempre la morale epicurea una morale * da sfaccendati chiusi nei loro orticelli ", non approvava nemmeno le morali troppo austere, come quella degli stoici, che per altra via riusciva anch'essa a morale da «solitarî » e non da uomini viventi in repubblica. Lo stoicismo predica, sí, nn giusto eterno e immutabile e vuole l'onesta norma delle azioni umane; ma convelle la natura umana, la disumana, l'annienta e la induce alla disperazione pretendendola affatto insensata alle passioni, sconoscendo le utiliti e necessita di natura corporea, inculcando quella massima «piú dura che ferro», ehe i peccati siano tutti eguali e che tanto pecchi colui che batte uno schiavo quanto chi uccide il padre. Anehe il giansenismo gli dové suscitare nell'animo gli stessi dubbî, se lamenta che « in odio della probabile, 
s'irrigidisca in Francia la cristiana morale». Non i filosofi solitarî, ma quelli politici bisogna seguire, e principalmente i platonici, i quali riconoscono che si debbono, non gia sradicare, ma moderare le umane passioni e farne umane virtú. Cosí dalla ferocia, dall'avarizia, dall'ambizione, che sono i tre vizî che corrono attraverso tutto il genere umano, la Provvidenza trae la milizia, la mercatanzia e la corte, ossia la fortezza, l'opulenza e la sapienza delle repubbliche: di quei tre grandi vizî, che distruggerebbero l'umana generazione sulla terra, plasma la civile felicità.

Anche circa le utilita il Vico osserva che «ex se» non sono lué buone né cattive ("neque turpes neque honestce"), tali diventando solamente nel loro rapporto con la coscienza morale ( sed earum incequalitas est turpitudo, aqualitas autem honestas »). Nella sua scienza empirica, propugna contro il Grozio un ius naturate prius, al quale si riferiscono la tutela della propria vita e la procreazione ed educazione della prole, e lo ravvicina all'żż’́popov degli stoici. Che esso non abbia autorità morale è provato da ciò, che il diritto che gli succede nell'ordine genetico, il ius naturale posterius (da Giustiniano definito: "quod naturalis ratio inter omnes homines constituit tt apud omnes gentes perceque custoditur ") è prius iure, vince nel conflitto tra i due e segna quello anteriore della nota d'immutabilità. Ora, sebbene questo diritto naturale primo sia esemplificato e definito in modo soltanto empirico, che cosa mai esso è, in abbozzo, se non il mero diritto, il diritto non ancora eticizzato?

Ma dore piú propriamente si annida, presso il Vico, il diritto come distinto dalla morale è nel concetto del certo: parola questa usata da lui in molteplici significati, non bene sceverati né messi in armonia né dedotti l'uno dall'altro, benché tutti un po' confusamente si raccolgano 
come abbiamo visto, sotto quello grenerale della forma spontanea dello spirito in quanto distinta dalla forma riflessa. Il certo, nella sua accezione pratica, vuol dire, tra l'altro, opposizione al vero della volontà, ed è, insomma, la forza di fronte all'equita e alla ginstizia, l'autoritì di fronte alla ragione, la mera volonta di fronte alla volontà morale. Tale distinzione nasce piuttosto nella nostra mente, che non sia nelle parole del Vico; il quale distingue e non distingue, e, affermato, per es., che «certum ab auctoritate est, verum a ratione », subito dopo afferma altresí che * anctoritas cum ratione omnino pugnare non potest, nam ita non leges essent, sed monstra legum ». Tuttavia, per effetto di questa trattazione del ce ro, la Scienza nuova gli parve che contenesse una filosofia dell'antorita; la quale (soggiungeva) " è il fonte della giustizia esterna, che dicono i morali teologi ». E, cioè, connetteva il concet to del certo con la distinzione e la terminologia dell'esterno e dell' interno, gia in uso nella morale scolastica, e che, adoperate circa quel tempo da Cristiano Tomasio, senza troppo merito filosofico di costui erano destinate a dare avviamento all'indagine filosofica dei rapporti tra diritto e morale.

Altro ma affine significato del certo pratico nel Vico è quel che si chiama la lettera delle leggi, formula legum: lettera che può stare in contrasto con la ragione e eon lal morale, e tuttavia ha la sua peculiare efficacia: "dure iex, sed certe; durum, sed scriptum est». 亡, insomma, il valore della legge in quanto legge, la quale, destituita di un vero contenuto etico, serba pur sempre il valore che le viene dall'imperio della volonta. "Il certo delle leggi (scrive il Vico) è un'osenrezza della ragione unicamente sostenuta dall'autorità, che le ci fa sperimentare dure nel praticarle e siam necessitati praticarle per lo dir lor certo, che in buon latino significa 
particolarizzato, o, come le scuole dicono, individuato . Il Vico intravrede in qualche modo perfino il carattere individuale, che è all'origine di ogni legge. Il "legibus, non exemplis iudicandum» è precetto relativamente tardo: le prime leggi furono, per l'appunto, «exempla", eastighi esemplari. Dagli esempî reali derivarono quelli ragionati, dei quali si valsero e si valgono la logica c la rettorica; e, quando poi furono intesi gli universali intelligibili, si riconobbe che la legge dovesse avere carattere di universalità.

La società primitiva che il Vico ritrae, è, nel suo aspetto giuridico, quasi il mito del diritto mero ossia della forza pratica. Un tempo furono nomini di sformate forze di corpo ed altrettanto stupidi d'intendimento, i quali stimavano divina ogni forza superiore alla loro umana (e questo era il loro diritto); e gli dèi non altro che esseri piú forti, ai quali erano costretti a piegarsi, pur fremendo, come Polifemo, che se ne avesse avuto la facolta avrebbe combattuto col medesimo Giove, o come Achille, che ad Apollo diceva che, se le sue forze fossero pari, non si sgomenterebbe di venire a tenzone con lui. Fu consiglio della Provvidenza che codesti uomini feroci, non addomesticati dall'impero della ragione, temessero almeno la divinità della forza, e da essa estimassero la ragione. E su ciò si fonda il principio della giustizia esterna della guerra. Ma il mito dell'età della forza non può avere il rigore del concetto filosofico; e perciò quei forti sono dal Vico, per un altro verso, considerati come eticamente i migliori : «fortissimi » e optimi », termini identici; il loro diritto non è il vero, il diritto razionale, ma nemmeno è il puro certo: è il vero « ex certo mixtum ». Senonché, lo stesso affermato miscuglio e predominio del certo nel vero postula il concetto del puro certo, dal Vico sottinteso. 
Quando il Vico rimprovera il Grozio e gli altri giusnaturalisti di avere cominciato la storia dalla metà in giú, dalle eta civili, e trascurato il periodo precedente, il rimprovero, per ciò che si attiene alla filosofia della pratica, si traduce in quello di avere dimenticato il momento ideale della forza per guardare unicanente alla giustizia, all'equità e alla moralità. Il momento della forza, che costituisee l'altra ossia la prima «metà ", fu invece il campo chiuso dell'Hobbes e, prima di lui, del Machiavelli, e, prima ancora, di Epicuro, i quali trattarono di esso solo, « con empietà verso Dio, con iscandalo verso i principi e con ingiustizia verso le nazioni ». Donde è agevole ricavare che la confutazione che il Vico fa degli utilitaristi e teorici della forza, $\dot{e}$, in pari tempo, riconoscimento e accettazione della esigenza che essi rappresentavano e che avevano il solo torto di svolgere in modo unilaterale e astratto: il suo « stato ferino» somiglia per piú rispetti all' hobbesiano, con la differenza che da questo l'umanità si salva mercé la riconosciuta utilità, e dal vichiano si salva mercé la coscienza religiosa e morale. Pure il Vico non professò, per questa parte, nessuna gratitudine all' Hobbes e allo Spinoza, al Machiavelli o a Epicuro, perché gli parve di avere trovato in un autore classico tutto il materiale e lo stimolo che gli giovava, tutto il contrappeso di cui la filosofia platonica aveva bisogno. L'autore fu uno dei suoi quattro, e quello appunto di cui si è avvertito di sopra che restava a noi ancora da vedere l'uso che il Vico ne fece: Tacito. Il quale, a suo dire, con mente metafisica incomparabile contempla l'uomo qual è, laddove Platone lo contempla quale dev'essere; e come Platone con quella scienza universale si diffonde in tutte le parti dell'onestá, cosí Tacito discende a tutti i consigli dell'utilita, acciocché tra gl'infiniti irregolari eventi della milizia e della fortuna l'uomo sapiente di pratica si conduca bene. AI 
congiungimento nel suo spirito del filosofo greco e dello storico romano (interpetrato da lui, come è agevole scorgere, nel modo consueto presso i politici *tacitiani del Seicento) il Vico attribuí l'essere riuscito ad abbozzare una propria idea della storia eterna, « onde se ne formasse il sapiente insieme di sapienza riposta, qual è quel di Platone, e di sapienza volgare, qual è quel di Tacito». Da Tacito, insomma, egli avrebbe rieevuto la spinta al suo gran lavoro, che fu di rendere conereto l'ideale, e d'inserire (come dieeva, adattando un detto ciceroniano) la repubblica di Platone nella feccia di Romolo. 
LA STORICITA DEL DIRITTO

C

Uome lo spirito conoscitivo passa dal sentire senza avvertire all'avvertire con animo perturbato e commosso e indi al riflettere con mente pura; cosí, analogamente, lo spirito volitivo passa dalla ferinita al certo pratico e da questo al vero. Nella correlativa scienza empirica il passaggio è press'a poco quello dallo stato ferino all'eroico o barbarico e dall'eroico al civile. Tutte le manifestazioni della vita si conformano a questi tre tipi sociali: donde tre spezie di nature, tre spezie di costumi, tre spezie di diritti e quindi di repubbliche, tre spezie di lingue e di seritture, tre spezie di autorita, di ragioni, di giudizî, tre sètte di tempi. Per quanto il Vico sia confuso e talvolta contradittorio nel determinare i particolari delle varie corrispondenze, il suo pensiero generale è chiaro. Dove la riflessione è scarsa e la fantasia gagliarda, sono anche gagliarde le passioni, violenti i costumi, aristocratici ossia feudali gli stati, sottoposte alla rigida autorita paterna le famiglie, dure le leggi, simbolici i procedimenti dei negozî giuridici, metaforici i linguaggi, geroglifiche le scritture. Per contrario, dove la riflessione predomina, la poesia si dilegua o si riempie di filosofia, i costumi si fanno miti, le passioni regolate, i popoli assumono i governi, i componenti 
delle famiglie sono anzitutto cittadini dello stato, le leggi si compenetrano di equità, le procedure si semplificano, i linguaggi si sfrondano di metafore, le scritture diventano alfabetiche. Forme miste, quali le vagheggiano artificiosamente alcuni politici, sarebbero mostri; e sebbene si osservino forme mescolate naturalmente, ossia ritenenti il vezzo delle primiere, ciascuna forma per la sua unità si sforza sempre, quanto piú paò, di scacciare dal suo subbietto tutte le proprietà di altre forme.

Quale dei varî tipi sociali sta a fondamento degli altri e porge il criterio per giudicarli? o quale è il criterio e la misura per gindicarli tutti quanti? Una siffatta domanda, per il Vico, non ha senso. Ciascuno di quei tipi ha la propria misura in sé stesso. I governi (egli dice) debbono essere conformi alla natura degli uomini governati: la scuola dei principi è la morale dei popoli. Si può inorridire innanzi alla guerra, al diritto del piú forte, alla riduzione dei vinti a sehiavi, cioè a cose che ripugnano ai nostri costumi ingentiliti; ma la societa, che si esplicava con quei costumi, era necessaria e perciò buona. La divinità della forza, come si è detto di sopla, teneva il posto e compieva l'ufficio del non ancora possibile impero della ragione. Vengono di poi i tempi della ragione umana tutta spiegata; e gli uomini non si stimano piú secondo la forza, ma si riconoscono eguali nella natura ragionevole, che è la propria ed eterna natura umana. Altri tempi, altri costumi, e buoni non meno, ma non piú, dei primi.

Tanto varrebbe domandare la misura comune di questi varî tipi sociali, quanto se si domandasse quale sia la vera età della vita individuale, la misura comune della fanciullezza, della giovinezza, della virilita, della vecchiaia. Paragone che, per l'appunto, il Vico stesso mette innanzi. Come i fanciulli tutto scelgono secondo il capriccio e si comportano con violenza, gli adolescenti vigoroggiano per 
la fantasia, gli adulti guidano le cose con piú pura ragione e i vecchi con solida prudenza; cosi al genere umano, infermo, solitario e indigentissimo nelle sue origini, convenne crescere dapprima in isfrenata libertà, poi ritrovare i necessarî, utili e comodi della vita con l'ingegno e con la fantasia (che fu il secolo dei pocti); e, infine, coltivare la sapienza con la ragione (che fu il secolo dei filosofi). Parimente, il diritto naturale nacque dapprima con lcggi, per cosi dire, di giusta libidine e di giusta violenza; poi fu rivestito con alcune favole di giusta ragione; infine, si affermò apertamente nella sua schietta ragione e generosa verità.

Con siffatto modo di considerare e giudicare stati, leggi e costumi, il Vico respingeva un'altra delle dottrine o delle pretese capitali del giusnaturakismo: quell'astrattismo e antistoricismo, che abbiamo ricordato a suo luogo, e del quale era conseguenza la concezione di un diritto naturale, che stia di sopra al diritto positivo, e percio una sorta di codice eterno, una legislazione perfetta, non attuata ancora pienamente ma da attuare, i cui lineamenti traspaiono con molta nitidezza nelle opere dei giusnaturalisti attraverso il tenue velame dottrinale e filosofico. Codice eterno, che era poi, nella sua parte effettuale, un codice contingente e transitorio, o almeno la proposta di un codice conforme alle tendenze riformistiche e rivoluzionarie di quegli serittori, piuttosto che filosofi, pubblicisti.

Il Vico si spaceia del codice ideale eterno senza averne l'aria: prontissimo, anzi, a riconoscere che il a ius naturale philosophorum » ì eterno nella sua idea e severissimamente stabilito «ad rationis aterno libellam ». Ma dall'eternitì concessagli a parole e per osscquio alla vecchia filosofia scolastica e triadizionale, della quale qua e la egli risentiva l'efficacia, passa a negargli di fatto l'eternità e il carattere soprastorico, perché, invece di metterlo sopra e fuori la 
storia, lo colloca al posto che gli spetta, dentro la storia. Il diritto della violenza o eroico, cangiatosi nel diritto incivilito, giunge via via a un certo termine di chiarezza, al quale per la sua perfezione altro non rimane che alcuna setta di.filosofi lo compia e fermi con massime ragionate sull'idea di un giusto eterno; e questo raziocinamento e sistemazione è il « ius naturale philosophorum », estrema forma dello svolgimento storico del diritto e non già regola perpetua di esso: risultamento, non misura. Di qui l'accusa del Vico al Grozio che, per avere scambiato il \& ius naturale philosophorum », il diritto composto di massime ragionate da moralisti e teologi e in parte da giuristi, col * ius naturale gentium » (nella terminologia groziana: per avere scambiato il diritto naturale con una forma di diritto arbitrario o positivo), fraintese i giureconsulti romani, i quali intenderano parlare solamente di questo secondo, e percio propose correzioni e mosse loro censure i cui colpi vanno a cadere nel vuoto.

Il codice eterno, considerato intrinsecamente, è un'utopia; e poiché la prima e maggiore delle utopie fu la Repubblica platonica, conviene, per meglio determinare il punto di cui si tratta, osservare il comportamento del Vico rispetto alla costruzione politica platonica. A dare ascolto alle sue parole, la Repubblica platonica sarebbe stata un altro dei tanti incentivi e modelli che egli avrebbe avuti a concepire la Scienza nuova. Dallo studio di Platone incomineiò a destarsi in lui, senz'avvertirlo, \& il pensiero di meditare un dritto ideale eterno che celebrassesi in una città universale nell'idea o disegno della providenza, sopra la quale idea son pure fondate tutte le repubbliche di tutti i tempi, di tutte le nazioni: che ela quella repubblica ideale, che in conseguenza della sua metafisica divina doveva meditar Platone ». Doveva, ma non lo poté fare "per l'ignoranza *, in cui egli era, " del primo 
uomo caduto $\gg$; cioè dell'originario stato ferino e della sapienza, che gli successe, affatto poetica o volgare: ignoranza in cui fu mantenuto per un errore comune delle menti umane che misurano da sé le nature non ben conosciute d'altrui, di guisa che egli innalzò le barbare e rozze origini dell'umanita gentilesca allo stato perfetto delle sue altissime divine cognizioni riposte, e sapientissimi di tal sapienza riposta immaginò quei primi uomini che furono invece, nella realta, « bestioni tutti stupore e ferocia ». In conseguenza di quešt'errore erudito Platone, in cambio di meditare sulla repubblica eterna e sulle leggi del giusto eterno con le quali la Provvidenza ordinò il mondo delle nazioni e lo governa con le bisogne comuni del genere umano onde esse si reggono sul comune senso di tutta l'umana generazione, "medito in una repubblica ideale ed in un pur ideale gi usto ", col quale le nazioni non si conducono punto. $\mathrm{E}$, anzi, se mai, dovrebbero discostarsenc e purgarsene, perché tra quelle determinazioni di repubblica perfetta se ne trovano alcune disoneste e da aborrire, com'è la comunanza delle donne. Cosicché, il Vico accettava da Platone l'idea di una repubblica eterna, sconvolgendola da cima a fondo con la soggiunta riserva: che la vera repubblica eterna non è l'astratta platonica, ma il eorso storico in tutti i suoi varî e successivi modi, dai bestioni non esclusi a Platone compreso. Di codesta, che è la * generis humani respublica», la * magna goneris humani civitas », la "respublica uriversa , egli intende studiare \& formam, ordines, societates, negotia, leges, pecata, ponas et scientiam in ea tractandi iuris », e come tutte queste cose si venissero svolgendo « a suis usque primis humanitatis originibus, divina providentia moderante, moribus gentium ac proincle auctoritate », cioè \& per varic utilitatum et necessitatum humanarum rudimenta, sive adeo per ipsarum sponte rerum oblatas occasiones ». La « grau 
citti delle nazioni fondata e governata da Dio » è nient'altro, dunque, che la Storia.

Negando il fissamento del codice e la delineazione della società modello, non si vuole, per altro, dichiarare impossibile una pratica della scienza concepita dal Vico, cioè, della Scienza nuova considerata sotto il triplice aspetto di storia ideale, storia tipica e storia storica. Ogni veriti ha la sua pratica, ossia le sue conseguenze pratiche; e pensare in un modo o in un altro la natura e lo srolgimento dell' umanità importa condursi, praticamente, nell' un modo o nell'altro. Chi, per es., crede alla docile innocenza dei popoli selvatici, andrà verso quelli $\mathrm{col}$ viso sorridente, con la bocca piena di buone parole, reeando in mano l'abbici e il catechismo dei diritti e doveri; chi crede invece ai « bestioni » vichiani, adotterà procedimenti alquanto piú severi e, forse, il ferro e il fuoco. Chi pensa, come il Vico, che « i costumi valgano piú delle leggi », e, insieme, che « i costumi non si cangino d'un tratto ma per gradi e in lungo tempo », non sarà incline al facile legiferare e non s'illuderà di potere plasmare a nuovo l'umanità sopra un modello soggettivo. Codesta pratica, a ogni modo, non è piú teoria, ma azione; e quando si vuole ridurla a teoria, o si fa un miscuglio mostruoso di determinazioni nccessarie e di determinazioni contingenti, o, nello scansare taie errore e sforzarsi di raggiungere una forma rigorosamente dottrinale della pratica, si riottiene né piú né meno che la teoria scientifica stessa, dalla quale la pratica derivava.

Il Vico ebbe il pensiero di far seguire alla sua Scienza nuova una Pratical della Scienza nuova; e anzi nella prima redazione italiana dell'opera proponeva due " pratiche $» 10^{\circ}$ una nuova arte critica, da scrvire di fiaccola per distinguere il vero nella storia oscura e favolosa; $2 .^{\circ}$ un'arte come diagnostica, per determinare i gradi di necessità o utilità delle cose umane, e, come ultima con- 
seguenza, ottenere il modo di conoscere per segni indubitati lo stato delle nazioni. Arte critica e diagnostica che, chi ben consideri, confluivano in una sola: nella migliore conoscenza che, mercé i principî stabiliti dal Vico, era dąto conseguire circa la vita passata e presente delle nazioni.

Tale concetto è ripetuto e schiarito in altri luoghi del medesimo libro. Le scienze, discipline e arti fin oggi svolte (dice il Vico) concernono oggetti particolari; la Scienza nuova, che investiga i principî stessi dai quali escono tutte le discipline, è invece al caso di stabilir l'axun o stato di perfezione dell'intero complesso, e i gradi c gli estremi per i quali e dentro i quali l' umanità, come ogni altra cosa mortale, deve correre e terminare; cosicché si apprendono mercé quella scicnza le pratiche come una nazione, sorgendo, possa pervenire allo stato perfetto, e come, decadendo da esso, possa sollevarvisi di nuovo. Lo stato di perfezione consisterebbe nel fermarsi le nazioni in certe massime cosi dimostrate per ragioni costanti come praticate coi costumi umani, nelle quali la ragione riposta dei filosofi desse la mano e sorreggesse la sapienza volgare delle nazioni, e in cotal guisa convenissero i piú riputati delle accademie con tutti $i$ sapienti delle repubbliche ( $i$ filosofi, cioè, e gli uomini di stato), e la scienza delle divinc e umane cose civili ossia della religione e delle leggi, le quali sono una teologia e una morale pomandata che si acquista per abiti, fosse assistita dalla scienza delle divine e delle umane cose naturali, che sono una teologia e una morale ragionata, che s'acquista coi raziocinî; talché trarsi fuori di si fatte massime sarebbe il vero errore o divagamento, non di uomo ma di fiera. La pratica della Scienza nuova, dunque, come si vede chiaro dal programma cosí tracciato, non era altro che il riassunto o il duplicato della scienza stessa, della quale mettcra 
in risalto $\mathbf{i}$ due elementi capitali: la sapienza spontanea e quella riflessa, il certo e il vero, e la necessita di tener conto di entrambi.

Anni dopo, in una delle elaborazioni che il Vico fece della seconda Scienza nuova (quella del 1731), ricompare il pensiero e la parola di una pratica di essa scienza, come suona il titolo di uno speciale paragrafo conclusivo, che egli si proponeva di aggiungere all'opera. * Tutta quest'opera (cosí cominciava quel paragrafo) è stata finora ragionata come una mera scienza contemplativa d'intorno alla comune natura delle nazioni: però sembra per questo istesso mancare di soccorrere alla pr adenza umana onde ella si adoperi perché le nazioni, le quali vanno a cadere, o non ruinino affatto o non s'affrettino alla loro ruina; ed in conseguenza mancare nella pratica, qual dee essere di tutte le scienze che si ravvolgono d'intorno a materie le quali dipendono dall'umano arbitrio, che tutte si chiamano attive». Or bene: in che mai consistera codesta pratica? * Cotal pratica ne può essere data facilmente da essa contemplazione del corso che fanno le nazioni, dalla qual avvertiti i sapienti delle repubbliche (gli uomini di stato) e i loro principi, potranno con buoni ordini, leggi ed esempli richiamar i popoli alla loro $\dot{\alpha} x_{i} \dot{y}$ o sia stato perfetto . In altri termini: uomo avvisato, mezzo salvato; la contemplazione è la sola pratica che la Scienza nuova possa dare; l'altra metà della salvazione dipende non già dall'avvisante cioè dal pensiero, ma dall'avvisato, cioc dal fare. Al Vico non viene neppure in mente di porsi a determinare * gli ordini, le leggi e gli esempli , che faccia d'uopo adottare in questo o quel tempo e luogo, perché codesta non sarebbe impresa da filosofo; come infatti riconosce esso stesso subito dopo, chiaramente, col dire: "La pratica, la qual ne possiamo dar noi da filosofi, ella si può chiudere dentro dell'ac- 
cademie s; ch'è quanto dire nel puro campo della scienza e della cultura.

Sarebbe, di certo, temerario pretendere di conoscere con sicurezza le ragioni per le quali il Vico, come aveva omesso nella prima edizione della seconda Scienza nuova le dichiarazioni circa la pratica che erano nella prima, tralascio nel manoscritto dell'ultima edizione dello stesso libro anche il capitoletto sulla Pratica. Ma sara lecito per lo meno congetturare che la ragione principale fosse nell'avvertita vacuità di quel capitoletto, il quale, promettendo una pratica, non la dava, e finiva col confessare che o non si poteva dare o era stata gia data con la teoria stessa. 


$$
\text { . }
$$




\section{$\mathrm{X}$}

\section{La Provvidenza}

\section{L}

que il loro corso; e il principio che governa il corso delle nazioni è la Provvidenza. Sotto questo aspetto la Scienza nuova si può definire una Teologia civile ragionata della provvidenza divina. Tra le discipline storiche era segnata dal Bacone una "historia Nemeseos ». Ciò che il Bacone enunciava come poco piú che nudo titolo, il Vico formolò come effettivo problema e svolse come teoria. I filosofi, secondo lui, quando non avevano addirittura disconosciuto come materialisti e fatalisti la Provvidenza, l'avevano considerata solamente sull'ordine delle cose naturali (epperò chiamavano "teologia naturale " la metafisica), confermando quell'attributo di Dio con l'ordine fisico che si osserva nei moti dei corpi, per es. delle bfere e degli elementi, e con la cagione finale osservata sopra le altre minori cose naturali. Importava, invece, ragionare la Provvidenza « sull'cconomia delle cose civili».

Taluno dei primi interpetri ebbe a notare (e l'osservazione è stata rinnovata in séguito non poche volte) che il Vico adoperò promiscuamente la parola " provvidenza " in significato soggettivo e in significato oggettivo: ora come persuasione che hanno gli uomini di una divinità provvi- 
dente che regga i loro destini, ed ora come l'efficacia stessa di questa provvidenza. La duplice o triplice accezione di un medesimo vocabolo nella terminologia vichiana non è cosa che, a questo punto, ci possa piú stupire, perché gia piú volte finora ci siamo dovuti industriare a sciogliere le sue omonimie o a unificare le sue sinonimie. E percio ammettiamo subito che uno dei significati di « provvidenza » presso il Vico può essere, anzi è per l'appunto, quello della persuasione circa la provvidenza, ossia l'idea che l'uomo ha di Dio, dapprima nella forma del mito, dipoi in quella pura e ragionata della filosofia. Le antiche nazioni gentili (egli dice) " incominciarono la sipienza poetica metafisica di contemplare Dio per l'attributo della sua provvidenza », sulla quale furono fondati gli auspicî e la divinazione. Senza di essa, dunque, non si forma nell'uomo la sapienza, che è coscienza dell'intinito; non sorge liı moralità, ch'è timore e riverenza del potere superiore che governa le cose umane. Ma la provvidenza, in tale significato, non dà luogo a nuovo discorso, dopo di quello già fatto da noi a proposito cosí del mito come dei rapporti tra morale e religione.

Passando, dunque, senz'altro, alla Provvidenza nel secondo significato, ossia al suo vero e proprio concetto, ci sembra opportuno prescindere per qualche istante dal Vico e fornire alcuni schiarimenti dottrinali.

$\dot{E}$ comune osservazione che altro è produrle un fatto, altro conoscere il fatto prodotto. La conoscenza di ciò che realmente un fatto è, si ottiene talora, nella vita dell'individuo, dopo parecchi anni, nella vita dell'umanità dopo parecchi secoli. Coloro medesimi che sono i diretti agenti di un fatto, non ne banno di solito la conoscenza o l'hanno assai imperfetta e fallace; tanto che sono passate in proverbio le illusioni, che, come si dice, accompagnano l'attività degli uomini. Il poeta crede di cantare la puriti 
ed effettivamente canta la lascivia; crede di cantare la forza e canta la debolezza; crede di essere terribilmente pessimista ed è fanciullescamente ottimista; crede di essere Satana ed è un brav'uomo inoffensivo. Non meno s'ingannano i filosofi; e dei loro inganni non dovremo, in verità, andar lontano a cercare esempî, perché tanti e tanti ce ne viene porgendo proprio il filosofo che stiamo studiando: uno di coloro che maggiormente s'illusero sulle reali tendenze dei proprî pensieri. E s'inganna l'uomo politico che, assai spesso, credendo e professando di lottare per la libertà, è semplice aiutatore di reazione, o credendo di servire alla reazione, incita a ribellarsi e serve alla libertà. E via discorrendo. Illusioni spiegabilissime, perché gl'individui e i popoli, nel ferrore del produrre o appena uscenti da quel fervore, possono forse esprimere il loro stato d'animo, ma nou farne quella critica che è il racconto storico; onde, quando non si rassegnano a tacere e ad aspettare, narrano di sé stessi storie fantastiche, verita e poesia commiste. Anzi, in questa dimostrata difficoltà di conoscere l'agire nell'agire è uno dei motivi della saggia raccomandazione a parlare il meno possibile di sé medesimi, e della diffidenza che si prova per le autobiografic e i libri di menorie, curiosi e anche, se si vuole, importanti, ma che non porgono mai la schietta verità storica dei fatti narrati.

Le opere umane ci ginngono, per tal modo, avvolte nei fumi delle illusioni che si sollevano dagl'individui. E lo storico superficiale si ferma all'involucro e prende a raccontare come le cose siano andate, facendosi portavoce di quelle illusioni. A questo modo la storia della poesia si viene conformando come il racconto delle intenzioni, delle opinioni, dei fini del poeta o di quelli che gli attribuirono i suoi contemporanei; la storia della filosofia, come l'aneddotiea dei sentimenti, delle bizze, e dei fini pratici dei fi- 
losofi; quella politica, come un tessuto d'intrighi, di bassi interessi, di pettegolezzi, di miserie. Ma non appena un piú cauto o diverso ingegno storico si avvicina a quelle storie, il primo atto ch'egli compie è di soffiare sulla nebbia, spazzare via gl'individui e le loro illusioni e guardare direttamente le cose, quali si sono prodotte nella loro successione oggettiva e nella loro origine sopraindividuale. La storia vera e reale emerge allora di là dagl'individui, come un'opera che si compia dietro le loro spalle: opera di una forza diversa dagl'individui agenti: Fato, Caso, Fortuna, Dio. Gl'individui, che prima erano tutto e riempivano la scena coi loro gesti o coi loro gridi, ora, in questa seconda guisa di storia, sono meno che nulla, e i loro atti e gridi, destituiti di seria cfficacia, destano riso o pietà. Si guarda atterriti il Fato che li domina, si stupisce alle strane combinazioni del Caso e ai capricci della Fortuna, si adorano i disegni imperscrutabili della Provvidenza divina. Di codeste forze gl'individui appaiono a volta a volta l'inerte materiale, i leggieri giocattoli, i ciechi strumenti.

Senonché una piú profonda considerazione va oitre anche questa seconda veduta della storia. La pietà che sembrano destare gl'individui, la comicita che suscitano, in effetti non è meritata da essi ma dalle loro immaginazioni, o, piuttosto, da coloro che le scambiano per verita. La storia reale è fatta dalle opere e non dalle immaginazioni e illusioni; ma le opere sono poi compiute dagl'individui, non certamente in quanto sognanti, ma, appunto, in quanto operanti; non nella frivolezza del loro opinare, ma nell'ispirazione del genio, nel sacro furore del vero, nel santo entusiasmo dell'eroismo. Fato, Caso, Fortuna, Dio sono spiegazioni che Lanno tutto il medesimo difetto, che è di separare l'individuo dal suo prodotto, e, invece di cacciare via, come si argomentano, il capriccio o l'arbitrio individuale dalla storia, inconsapevolmente lo rafforzano e lo 
moltiplicano. Capriccioso è il cieco Fato, il Caso stravagante, il tirannico Dio; epperò il Fato passa nel Caso e in Dio, il Caso in Fato e Dio, e Dio si converte nell' uno e nell'altro, tutti eguali e tutt'uno.

L'idea, che supera e corregge tanto la visione individualistica della storia quanto quella sopraindividualistica, è l'idea della razionalità della storia. La storia è fatta dagl'individui; ma l'individualità è la concretezza stessa dell' universale, e ogni azione individuale, appunto perché individuale, è sopraindividuale. Non vi è né l'individuo né l'universale come due cose distinte, ma l'unico corso storico, i cui aspetti astratti sono l'individualità priva di universalità e l'universalità priva d'individualità. Quest'unico corso storico è coerente nelle sue molteplici determinazioni, al modo di un'opera d'arte che è varia e una insieme e nella quale ogni parola si abbraccia coll'altra, ogni tono di colore si riferisce agli altri tutti, ogni linea si lega a ogni altra linea. A tale patto solamente è dato intendere la storia, che altrimenti resterebbe inintelligibile, come inintelligibili restano un discorso senza significato e una incoerente azione da folle.

La storia dunque non è opera né del Fato né del Caso, ma di quella necessità che non è fatalità e di quella liberta che non è caso. E poiché la veduta religiosa che la storia sia opera di Dio ha, sulle altre, il vantaggio e il merito d'introdurre una causa della storla che non sia né fato né caso, e perciò neppure piú propriamente causa ma efficienza creativa e spirito intelligente e libero, è naturale che, per atto di gratitudine verso questa veduta piú alta, non meno che per opportunita di linguaggio, si sia tratti a dare alla razionalita della storia il nome di Dio che tutto regge e governa, o della Provvidenza divina. A denominarla cosi, purgando in pari tempo la denominazione delle sue scorie mitiche, per le quali Dio e la sua 
provvidenza si corrompevano di nuovo in un fato o in un caso. Onde la Provvidenza nella storia ha, in quest' ultima sua forma logica, il duplice valore di una critica delle illusioni individuali, allorehé si presentano come la piena e sola realta della storia, e di una critica della trascendenza del divino. E si puo dire che nel punto di vista di essa si siano collocati e si collochino sempre, come per istinto, cioè anche senza fare professione di esplicita teoria, tutti gl'ingegni naturalmente forniti di quella particolare attitudine che si chiama senso storico.

Se ora, nel tornare al Vico, ricerchiamo quale soluzione egli desse al problema della forza che muove la storia, e quale contenuto preciso avesse in lui il concetto della provvidenza nel significato oggettivo, è agevole anzitutto escludere che la sua fosse quella Provvidenza trascendente e miracolosa, che aveva formato il tema dell'eloquente Discours del Bossuet. Agevole, sia perché egli in tutta la sua filosofia non fa mai altro ehe ridurre il trascendente all' immanente, e qui innumeri volte ripete che la sua provvidenza opera per vie naturali o (valendosi della terminologia della scuola) per cause seconde; sia perché sopra questo punto c'è, si può dire, fra gl'interpetri consenso generale.

Non meno insistente è la sua eritica del fato e del caso, o, come talora tripartisee, della fortuna, del fato e del caso. Egli avverte anche che la dottrina del fato si aggira in un circolo vizioso, perché la serie eterna delle cagioni, con la quale esso tiene einto e legato il mondo, pende dall'arbitrio di Giove e Giove è insieme soggetto al fato; onde c'è rischio che gli stoici restino avvolti in quella * eatena di Giove », con la quale vogliono trascinare le cose umane. Quei tre concetti, ai quali corrispondono le opportunita se si tratta di cose desiderate, le oceasioni se di quelle che arvengono oltre la speranza, e gli acci- 
denti se di quelle che si presentano oltre l'opinione, sono distinzioni piú che altro dell'apprendimento soggettivo, perché oggettivamente pertengono a un'unica legge, ła quale potrebbe chiamarsi altresi * fortuna , ove con Platone.si riconosca per signora delle cose umane l'opportunità; e tutte tre sono le manifestazioni e le vie della Provvidenza divina, che è intelligenza, libertà, necessità. Quello che fece il mondo delle nazioni « fu pur Mente, perché 'l fecero gli uomini con intelligenza; noṇ fu Fato, perclée'l fecero con elezione; non Caso, perché con perpetuità, sempre cosi facendo, escono nelle medesime cose ».

Il Vico lumeggia nei modi piú immaginosi quella commedia degli equivoci, che sono le illusioni circa i fini delle azioni che si compiono. Gli uomini credettero di salvarsi dalle minace del cielo fulminante col portare via le femmine nelle grotte per isfogare la libidine bestiale fuori dello sguardo di Dio; e, nel tenerle ferme cola dentro, fondarono i primi coneubiti pudici e le prime società; cioè i matrimonî e le famiglie. Si fortificarono in luoghi adatti (ol fine di difendere sé stessi e le loro famiglie; e, in realtà, con quel fortificarsi in certi luoghi, ponevano fine alla vita nomade, al divagamento ferino, e imparavano la cultura dei campi. I deboli e sregolati, ridotti alle estreme necessità dalla fame e dulle vicendevoli uccisioni, per campare la vita corsero a chiedere riparo in quelle terre fortificate facendosi famoli degli eroi; e cosí, senza stuperlo, vennero ad ampliare le famiglie da famiglie di soli figliuoli a famiglie anche di famoli e da queste a stati aristocratici e feudali. Gli aristocratici, feudatarî o patrizî, credettero di difendere e perpetuare il loro dominio quiritario sulle terre con l'usare la piú stretta ligidità verso i famoli o plebi che le lavoravano; ma a questo modo indussero i famoli, per loro difesa, a unirsi tra loro, svegliarono in essi la coscienza della propria forza, da plebe ne fecero uomini, 
e quanto piú fieramente i patrizî si stimarono patrizî e si sforzarono di mantenersi tali, tanto piú efficacemente concorsero a distruggere lo stato patrizio e a creare quello democratico. Cosi (dice il Vico) il mondo delle nazioni esce * da una mente spesso diversa ed alle volte tutta contraria e sempre superiore ad essi fini particolari ch'essi uomini si avevan proposti; de' quali fini ristretti fatti mezzi per servire a fini piú ampî, gli ha sempre adoperati per conservare l'umana generazione in questa terra .

Ma già da talune di queste parole del Vico si potrebbe ritrarre che egli tendeva talvolta a concepire gli uomini come coscienti dei proprî fini utilitarî e incoscienti di quelli morali. Il che condurrebbe logicamente a spiegare la vita sociale con esclusivi principî utilitarî, e la moralitì come un qualcosa di accidentale rispetto alla volontà uniana e perciò di non veramente morale: una formazione estrinseca piú o meno potente a tenere insieme gli uomini, o l'opera nascosta di una Provvidenza extramondana. L'utilitarismo s'insinua soprattutto in una pagina nella quale è detto che l'uomo, per la sua corrotta natura, essendo tiranneggiato dall'amor proprio pel quale segue principalmente la propria utilità e vuole tutto l'utile per sé e niuna parte pel compagno e non può porre in conato le passioni per indirizzarle a giustizia, nello stato bestiale ama solamente la sua salvezza; presa moglie e generati figliuoli, ama la sua salvezza con la salvezza della famiglia; venuto a vita civile, ama la sua salvezza con la salvezza della città; distesi gl'imperî sopra piú popoli, ama la sua salvezza con la salvezza della nazione; unite le nazioni in guerre, paci, alleanze e commerci, ama la sua salvezza con la salvezza di tutto il genere umano; e * in tutte queste circostanze ama principalmente l'utilità propria ». Per la qual ragione, 
« non da altri che dalla Provvedenza divina deve essere tenuto dentro tali ordini a celebrare con giustizia la famigliare, la civile e finalmente l'umana societa; per gli quali ordini, non potendo l'uomo conseguire ciò che vuole, almeno voglia conseguire ciò che dee dell'utilità, ch'è quel che dicesi giusto ». La pubblica virtú romana (scrive altrove) " non fu altro che un buon uso che la Provvedenza faceva di si gravi, laidi e fieri vizî privati, perché si conservassero le città ne' tempi che le menti degli uomini, essendo particolarissime, non poterano naturalmente intendere ben comune».

Senonché l'utilitarismo, come sappiamo, è affacto repugnante alla concezione etica del Vico, fondata sulla coscienza morale o sul pudore; e perciò queste sue affermazioni, che inconsaperolmente vi condurrebbero, non possono spiegarsi se non come effetto del turbamento che talora producera in lui la sopravvivenza del concetto trascendente e teologico circa la Provvidenza, e anche della poca chiarezza di pensiero, per la quale non gli riusciva di tenere ben distinto il concetto delle illusioni individuali da quello dei fini individuali e sostituiva talvolta il secondo dove avrebbe dovuto trattare solamente del primo. Se la provvidenza divina, l' * unità della religione d'una divinità provedente», è "l'unitả dello spirito che informa e dà vita al mondo delle nazioni », questa religiosità non può starsene al pensiero dell' inconsapevole indirizzamento dei fini individuali a effetti universali, ma deve esplicarsi nel dar vita e vigore al fini universali direttamente, e l'uomo sarà tutt'insieme utilitario e morale, o che s'illuda di essere morale dov'è utilitario o di esser utilitario dor'è effettivamente morale.

A ogni modo, e nonostante queste oscillazioni o piuttosto confusioni, concepire i fini particolari come veicolo degli universali e le illusioni come accompagnanti e coo- 
peranti con l'azione importa concepire dialetticamente il moto della storia e superare il problema del malc. Nel Vico, questo problema ha, infatti, pochissimo rilievo, tanto in lui dominava l'idea che la Provvidenza governi tutto; e perció quel che si chiama male, non solo gli si mostrava voluto dagli uomini sotto sembianza di bene (falsum sub veri specie, mala sub bonorum simulacris amplectimur), ma doveva logicamente svelarglisi come esso stesso una forma di bene, a quella guisa che bene era la barbarica forza costitutrice della prima societa. In qualche raro luogo dei suoi primi scritti nel quale gli accade di accennare a tali questioni, il Vico nota che noi altri uomini, a causa della nostra iniquità onde a nosmetipsos, non hanc rerum universitatem spectamus ", le cose che ci contrariano stimiamo male, "qua tamen, quia in mundi commune conferunt, bona sunt».

La concezione della storia diventa nel Vico veramente oggettiva, affrancata dall'arbitrio divino, ma non meno dall'impero delle piccole cause e delle spiegazioni aneddotiche; e acquista coscienza del suo fine intrinseco, che è d'intendere il nesso dei fatti, la logica degli avvenimenti, di essere rifacimento razionale di un fatto razionale. Gli studî storici, a quei tempi, non eranó tanto danneggiati dal primo errore (ché anzi la concezione teologica, fin dagli inizî del Rinascimento italiano, poteva considerarsi decaduta), quanto da quella forma di storia che appunto allora venne prendendo nome di prammatica, e che restringeudosi all'aspetto personale degli avvenimenti e non raggiungendo per questa via la piena realtà storica, cercava di darsi calore e vita mercé le riflessioni e gli ammaestramenti politici e morali. Un monumento di storia prammatica sorgeva nella stessa patria del Vico, contemporaneamente alla Scienza nuova: la Storia civile del regno di Napoli di Pictro Giannone, il quale era veramente 
l'uomo del suo paese e del suo tempo e scrisse un gran libro di polemica e anche, per certi rispetti, di storia, ma tale che, con la sua altezza, dà modo di segnare la tanto maggiore altezza dell'opera vichiana. Ben altro che astuzie di papi, vescovi e abati, e semplicita di duchi e imperatori, avrebbe saputo scoprire il Vico, se avesse dovuto narrare lui per filo e per segno le origini della proprietả e della potenza ecclesiastica nel Medioevo. F ben altro, come vedremo, egli scopri realmente nella storia, tutte le volte che prese a indagarne qualche parte. 



\section{$\mathrm{XI}$}

\section{RICORSI}

I

o spirito, percorsi i suoi stadî di progresso, e dalla sensazione innalzatosi successivamente all'universale fantastico e poi a quello intelligibile, dalla violenza all'equità, non può, in conformita della sua eterna natura, se non ripercorrere il suo corso, ricadere nella violenza e nel senso, e di la riprendere il moto ascensivo, iniziare il ricorso.

È codesto il significato filosofico del « ricorso » vichiano, ma non è il modo preciso in cui lo si trova espresso negli scritti del Vico, dove l'eterno circolo viene quasi esclusivamente considerato nelle storic dei popoli, come ricorso delle cose umane civili. La civilta va a terminare nella "barbarie della riflessione ", peggiore della prima barbarie del senso, clie era di una ficrezza generosa, laddove l'altra è vile, insidiosa e traditrice; e perciò è necessario che quella malnata sottigliezza d'ingegni maliziosi vada a irrugginire dentro lunghi secoli di una nuova barbarie del senso. Tuttavia, dai fatti storici e dallo schema sociologico bisogna estrarre e depurare il concetto del « ricorso », non solo per rendersi conto dell'assolutezza ed eternitả che il Vico gli attribuisce, ma anche per giustificare la rappresentazione storica e la legge sociologica che 
si fondano sopra di esso e da esso principalmente attingono la loro forza.

Le leggi di corsi e ricorsi, che erano state stabilite dai filosofi e politici greci e da quelli italiani del Rinascimento, si fondavano certamente anch'esse sopra qualche filosofia, ma assai superficiale; onde assunevano a loro obietto le estrinseche e vuote forme politiche, delle quali procuravano di determinare la suceessione sopra dati di esperienza o su vaghi raziocinî. Ma il Vico ha per suo obietto le forme di cultura, che abbracciano in sé tutti gli atteggiamenti della vita, l'economia e il diritto, la religione e l'arte, la scienza e il linguaggio; e, riportandole alla loro intima fonte, che è lo spirito umano, ne stabilisce la successione sccondo il ritmo delle elementari forme dello spirito. Per questo, tutta l'erudizione che si è spesa per ravvicinare i ricorsi vichiani alle teorie di Platone o di Polibio, del Machiarelli o del Campanella, riesce mediocremente inutile: tanto piú che il Vico (il quale, come sappiamo, pure fraintendendo spesso i suoi predecessori, non si può dire che volesse celarli, anzi, dove gli pareva scorgere riscontri e consensi, se ne pompeggiava) non sentí il bisogno di ricordarle o vi accennò con poca stima.

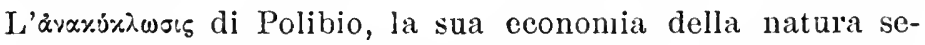
condo la quale si cangiano e tramutano e al medesimo punto gli stati ritornano, è sembrata quasi un'anticipazione della storia ideale eterna; pure il Vico mette Polibio insieme con gli altri, invitando i lettori a considerare « quanto [poco] i filosofi abbiano con iscienza meditato sui principî dei civili governi, e quanto [poco] con verita Polibio abbia ragionato sulle loro mutazioni ». Il Campanella connetteva i suoi circoli storici con leggi astrologiche; e il Machiavelli ecco come concepisce la catastrofe che inizia il ricorso: « Quando l'astuzia e malignita umana è venuta dove la può venire, conviene di necessità che il mondo 
si purghi per uno dei tre modi [peste, fame e inondazione, oltre quelli umani delle nuove religioni e linguaggi], aceiocché gli uomini, essendo divenuti pochi e battuti, vivano piú comodamente e divengano migliori ». Il solo precedente al quale il Vico quasi si gloria di riferirsi, è l'antichissima tradizione egiziana sulla successione delle tre età degli dèi, degli eroi e degli uomini, ehe interpetra in guisa tutta sua e riempie di eontenuto affatto nuovo.

Se la filosofia, che è nel fondo, conferisce forza alla teoria sociologica vichiana dei ricorsi, il materiale storico col quale è, per eosí dire, impastata, v'introduce qualehe debolezza. Il Vico ebbe pratica e predilezione particolare per la storia specialmente giuridica di Roma, donde mossero le sue indagini e alla quale si dedicò per piú anni; e questa storia, sia perché da lui meglio ricercata, sia per li sua stessa complessità, grandiosità e durata, finí per parergli la storia tipiea o normale, da servire di misura tutte le altre, e gli si confuse con la stessa legge del corso e ricorso. Roma gli offriva l'asilo di Romolo, cioè il passaggio dallo stato ferino all'ordinamento politico; le aristocrazie, monarehiche dapprima solo in apparenza, e poi neppure nell'apparenza; la democrazia, uscente dalla lotta contro l'aristocrazia e terminante nell'effettiva monarchia, cioè nella forma piủ perfetta della vita civile; e di qui, per proeesso degenerativo, la barbarie della riflessione ossia della civilta, che è incomparabilmente peggiore della prima e generosa barbarie, e, conseguenza di essa, una sceonda condizione di divagamento ferino, e la nuova barbarie, la nuova gioventú, il Medioevo. La storia di Roma, a mala pena generalizzata e integrata qua e la con quella della Grecia, si seorge nolle * degnita > vichiane che formolano le leggi della dinamica sociale. Gli uomini prima sentono il necessario, dipoi badano all'utile, appresso avvertiscono il comodo, piú innanzi si dilettano del pia- 
cere, quindi si dissolvono nel lusso, e finalmente impazzano in istrapazzar le sostanze. Ci vogliono prima uomini immani e goffi come i Polifemi, affinchẻ l'uomo ubbidisca. all'uomo nello stato delle famiglie, e per clisporlo a ubbidire alla legge nello stato futuro delle città. Ci vogliono i magnanimi e gli orgogliosi come gli Achilli, determinati a non cedcre ai loro pari, affinché sulle famiglie si costituiscano le repubbliche di forma aristocratica. Quindi si richicdono i valorosi e giusti, quali gli Aristidi e gli Scipioni Africani, per aprire la strada alla libertà popolare. Piú innanzi, personaggi appariscenti con grandi immagini di virtú accompagnata da grandi vizî, che presso il volgo fanno strepito di vera gloria, quali gli Alessandri e i Cesari, per introdurre le monarchie. Piú oltre ancora, i tristi riflessivi, quali i Tiberi, per istabilirle; e, finalmente, i furiosi, dissoluti e sfacciati, quali i Caligola, i Neroni, i Domiziani, per rovesciarle.

Per effetto di questo assottigliamento della storia romana a storia tipica, e insieme della corpulenza che la storia tipica acquista nella storia di Roma, la legge vichiana dei ricorsi è tutta rotta da eccezioni, assai piú frequenti e gravi che le medesime leggi empiriche non comportino; talché se il suo schema empirico fosse tutt'uno, come a lui sembrò, con la legge ideale dello spirito, parrebbe quasi ironia l'affermata costanza di esso nell'eternità e nei mondi infiniti. Il disegnato corso delle cose umane (egli scrive) non fecero, nell'antichità, Cartagine, Capua e Nuınanzia, le tre citta che minacciarono di disputare a Roma l'impero del mondo; perché i cartaginesi furono prevenuti dalla nativa acutezza africana, che piú aguzzarono nei commerci marittimi; i capuani dalla mollezza del cielo e dall'abbondanza della Campagna felice; i numantini, perché nel loro primo furore dell'eroismo furono oppressi dalla potenza romana, comandata da uno Scipione Africano, vin- 
citore di Cartagine, e assistita dalle forze del mondo. $\mathrm{E}$ dall'antichità saltando ai tempi moderni, gli americani correrebbero ora il corso delle cose umane, se non fossero stati scoperti dagli europei; Polonia e Inghilterra persistono stati aristocratici (tale stimava il Vico l'Inghilterra, perché, non come la Francia, monarchia assoluta), ma perverranno a perfettissime monarchie, se il corso naturale delle cose umane civili non sarà loro impedito da cagioni straordinarie. Neppur il Medioevo poteva considerarsi, secondo la mente del Vico, come un vero e proprio ritorno allo stato ferino, se si aprí con lo stabilimento della religione del vero Dio, del cristianesimo; né, a ogni modo, quel ritorno alla ferinità e alla barbarie sembra che sia la sola via che si offra alle nazioni, giunte alla loro $\grave{\alpha} x \mu \dot{\eta}$, al loro culmine. C'è l'altra che le nazioni corrotte perdano l'indipendenza e vengano sotto il dominio di altre migliori. Né, infine, la decadenza è inevitabile, se uomini di stato e filosofi, lavorando concordi, possono serbare la perfezione raggiunta e raffrenare la dissoluzione minacciante, e se difatti (come egli nota) le poche repubbliche aristocratiche che sopravvivevano ai suoi tempi quali residui del Medioevo (per es., Venezia), riuscivano a conselvarsi con arti di * sopraftina sapienza ». I suoi proprî tempi il Vico giudica di alta civiltà : una compiuta umanita (egli dice) sembra sparsa, oggi, per tutte le nazioni. Pochi grandi monarchi reggono il mondo dei popoli, e quelli ancora barbari o durano per la perdurante sapienza volgare di religioni fantastiche e fiere, o insiememente per effetto del temperamento naturale dei varî popoli. Le nazioni, infatti, soggette allo czar di Moscovia sono di mente pigra; quelle del chan di Tartaria, genti molli; i popoli sui quali regnano il negus di Etiopia e i re di Fez e di Marocco, deboli e parchi. Nella zona temperata il Giappone celebra un'umanità eroica, somigliante alla romana dei 
tempi delle guerre cartaginesi, fieri nelle armi, con una lingua che arieggia la latina (qui il Vico fraintendeva il ragguaglio di un missionario gesuita), con una religione feroce di dèi orribili tutti carichi d'armi infeste (e qui esagerava alquanto un passo del Bartoli); i cinesi, invece, con una religione mansueta, coltivano le lettere e sono umanissimi; umani ed esercitanti le arti della pace, i popoli del gran Mogol; i persiani e i turchi mescolano alla mollezza dell'Asia la rozza dottrina della loro religione, e i turchi in ispecie temperano l'orgoglio con la magnificenza, col fasto, con la liberalità e con la gratitudine. Umanissima per eccellenza l'Europa, composta in grandi monarchie e dove dappertutto si professa la religione cristiana, la quale insegna un'idea di Dio infinitamente pura e perfetta e comanda la carità verso tutto il genere umano. Il Vico ferma l'occhio sulle confederazioni dei cantoni svizzeri e delle provincie unite di Olanda (che gli ricordano le leghe etolie ed achee), e sul corpo dell'Impero germanico, sistema di città libere e di principi sovrani, che gli sembra quasi saggio di un grande stato aristocratico, il piú perfetto di tutti, forma ultima degli stati civili, perché non si puó intenderne altra superiore, riproducendo essa la prima, l'aristocrazia dei patrizî, ree sovrani nelle loro famiglie e uniti in ordini regnanti nelle prime città, ma riproducendola non piú barbarica, anzi sommamente civile. I' Europa sfolgora dappertutto di tanta -umanita, che vi si abbonda di tutti i beni i quali possono felicitare l'umana vita non meno pei piaceri della mente e dell'animo che per gli agi del corpo; e tutto ciò per virtú della religione cristiana che insegna verità sublimi, servita dalle piú dotte filosofie dei gentili e dalle tre maggiori lingue del mondo, l'ebraica, la greca e la latina, e riunente per tal modo la sapienza comandata con la ragionata, la piú scelta dottrina dei filosofi con la piú colta erudizione 
dei filologi. Codesta somma civilta, garantita dal cristianesimo, sarebbe andata o stava per andare incontro a un nuovo stato ferino? E difficile conoscere quel che veramente il Vico pensasse in proposito. C'è, tra i suoi versi, una canzone cupamente pessimistica; ma è una effusione giovanile e, a ogni modo, piuttosto che a decadenza sociale, accenna addirittura a un'imminente tine del mondo. Nelle sue lettere, si fa un triste quadro delle condizioni degli studî ai suoi tempi; ma non si spinge lo sguardo fuori di quel campo ristretto, a considerare la vita sociale $\mathrm{c}$ politica. D'altra parte, nell'ultimo suo scritto filosofico, nel De mente heroica, volgendosi a quelli che dicevano tutto essere ormai perfetto e non presentarsi nient'altro da fare, affermava che si era nel maggiol fervore di progresso: " Mundus iuvenescit adhuc; nam septingentis non ultra ab hinc annis, quorum tamen quadringentos barbaries percurrit, quot nova inventa? quot nova artes? quot nova scientia excogitata?... . Ma si potrebbe osservare che il De mente heroica è un'orazione accademica, e che forse per questo il Vico vi fece tacere i suoi dubbî o i suoi intimi convincimenti. In ogni caso, come adattare nella previsione di una imminente decadenza il sorgere di quel fatto provvidenziale che era la Scienza muova, la quale illuminava la vita delle nazioni e ne rendeva possibile la diagnosi e la cura? Tutto sommato, è probabile che il pensiero del Vico circa le sorti della società a lui contemporanea sia difficile tanto a cogliere perché, in verita, un pensiero determinato su quel punto a lui mancava, essendo il suo animo tratto in qua $c$ in la da diverse e opposte tendenze e agitato fra timori e speranze.

Se non fosse stata turbata dallo schema della storia romana, la teoria empirica dei ricorsi non sarebbe stata costretta ad accogliere tante e tanto gravi eccezioni, né si sarebbe impigliata in cosi angosciose perplessità, e avrebbe 
piú agevolmente allogato le osservazioni storiche dell'autore, e, insomma, si sarebbe presentata con tratti piú semplici e generali. Essa sarebbe consistita sopratutto nella determinazione e illustrazione del nesso tra epoche di prevalenza fantastica ed epoche di prevalenza intellettiva, tra spontanee $\mathrm{e}$ riflesse, onde dalle prime escono le seconde per potenziamento e dalle seconde, attraverso la degenerazione e la decomposizione, si torna alle prime. La storia politica mostra di continuo lo spettacolo di aristocrazie che, da forti che erano, si fanno vili e spregeroli, e cedono all'urto di classi meno affinate o addirittura rozze ma moralmente piú energiche, fintanto che queste, diventate a loro volta raffinate, raggiunta la piú alta fioritura delle idee storiche di cui portavano il germe, entrano in un periodo di decadenza e di fermentazione, dal quale esce una nuova classe dominatrice, giovanilmente barbara. E la stọia della filosofia mostra periodi positivistici e periodi speculativi, l'irrigidirsi delle soluzioni filosofiche nelle dottrine scolastiche e nei dommi, il ritorno alla mera osservazione del fatto singolo, e il rinascente processo speculativo. E la storia letteraria ci parla anch'essa di periodi realistici e idealistici, romantici e classicisti, di corruttela classica che è alessandrinismo e decaclentismo, e di barbarie romantica che da questo risorge. Ecco altrettanti casi di veri e proprî ricorsi vichiani. Ma poiché la natura dello spirito, messa a fondamento di questi cicli, è fuori del tempo ossia è in ogni istante del tempo, non bisogna esagerare la distinzione dei periodi; e, se quella legge deve avere una certa rigidezza, deve per altro serbare anche una certa elasticità. Non bisogna mai dimenticare che in ogni epoca, per aristocratica o democratica, romantica o classica, positica o speculativa che si dica, anzi in ogni individuo e in ogni fatto, è dato notare momenti aristocratici e democratici, romantici e classici, 
positivi e speeulativi, e ehe quelle distinzioni su grande scala sono quantitative e di comodo: il che non deve portarci né a sostenere quella legge a tutti i rischi, cadendo nell'artificiosità, né a eombatterla a oltranza, ricusando i servigi che gli schemi generali e approssimativi sogliono rendere.

Perciò, quando sia cosí intesa e corretta, non solo non ' c'è bisogno d'introdurre in essa quelle grosse e stridenti eceezioni che il modellamento sulla storia romana e sulla sua catastrofe finale faceva neccssarie, ma le aceuse mosse al Vico di troppa uniformità si dileguano. Vincenzo Cuoco, uno dei primi che presero a studiare con intelligenza l'opera del Vico, notava, a proposito e contro i ricorsi, che a la natura non si rassomiglia mai a sé stessa, ed è l'uomo ehe per comporre le sue osservazioni forma lc classi e i nomi ». Verissima sentenza, ma che si volesse applicare a questo caso, non varrebbe solo eontro i ricorsi viehiani, ma eontro ogni sorta di scienza umana di carattere empirico. Altri rimprovera al Vico di avere trascurato ordini di cause che hanno importanza grande nella storia, per es. il clima, le disposizioni naturali delle razze e dei popoli, gli avvenimenti straordinarî. Ma, lasciando stare ehe il Vieo fece menzione piú volte di tutte queste eose mettendo in rapporto i caratteri dei popoli e i climi con le forme $c$ vicende degli Stati e ricordando avvenimenti e eircostanze che affrettano il corso naturale ossia ordinario delle nazioni (come, tra l'altro, nel discorrere della storia greca); il vero è che egli non doveva tenerne conto, o non poteva indugiarvisi, perché il suo assunto coneerneva lc uniformità e non le differenze, o certe uniformità e non certe altre, ehe rispetto alle prime diventavano differenze trascurabili. Allo stesso modo (e il paragone è calzante ed è piú che un paragone) chi si faccia a notare i earatteri generali delle varie età della vita, della infanzia, della fan- 
ciullezza, dell'adolescenza e via dicendo, trascurerà di notare gli acceleramenti o ritardi di sviluppo secondo i varî climi o le varie razze o i varî accidenti. Nel medesimo gruppo di addebiti, veri e inopportuni insieme, rientra che il Vico abbia negato la comunicabilita e compenetrazione reciproca delle civilta col sostenere insistentemente che la civiltà nacque separatamente presso i popoli senza sapere nulla gli uni degli altri e perciò senza prendere esempio reciproco. Il quale addebito è stato controbattuto osservando che il Vico non manca di ricordare casi di efficacia di un popolo sull'altro e di trasmissione delle civiltả e dei loro prodotti (per es., della scrittura alfabetica dai caldei ai fenicî e da questi agli egiziani), e che, a ogni modo, la sua legge è non empirica ma filosofica e si riferisce alla spontaneità produttrice dello spirito umano. Senonché, ciò che è in discussione è appunto l'aspetto empirico e non quello filosofico della legge; e la risposta giusta sembra a noi, come si è già accennato, che il Vico non potesse e mon dovesse tenere conto delle altre circostanze, al modo stesso (per ripigliare l'esempio) che chi nello studiare le varie fasi della vita descrive le prime manifestazioni del bisogno sessuale nel vago fantasticare $o$ in altri fatti consimili della puberta, non tiene conto dell'iniziazione all'amore che gli adolescenti meno esperti possono ricevere dai piú esperti, quando l'assunto della ricerca concerna non le leggi sociali dell'imitazione ma le leggi fisiologiche dello sviluppo organico. E colui che affermasse che pur senza iniziazione e ammaliziamento il bisogno sessuale si risveglia egualmente e si procaccia soddisfazione, riaffermerebbe, senza dubbio, nient'altro che l'incontrastabile verità di un'antichissima novellina orientale che il Boccaccio inserí nel Decamerone, ma prollunzierebbe insicme il piú esatto riscontro alla famosa e tanto contrastata dignità vichiana. 
Né i ricorsi vichiani si oppongono di necessità, come spesso si è creduto, al concetto di progresso sociale. Si opporrebbero, se, invece di essere semplicemente uniformi, fossero identici, in conformità dell'idea, che si è affacciata nell'antichità e nei giorni nostri.a qualche cervello stravagante, dell'eterno ritorno delle cose singole e individuali. Il ripercorso del corso, il circolo eterno dello spirito, può o deve (sebbene il Vico non lo dica) pensarsi non solo diverso nel moto uniforme, ma continuamente arricchentesi e crescente su sé stesso, in guisa che la nuova epoca del senso sia in realta arricchita di tutto l'intelletto, di tutto lo svolgimento precedente, e cosí la nuova epoca della fantasia o quella della mente spiegata. La barbaric ritornata, il Medioevo, fu per tanti rispetti uniforme all'antica barbarie; ma non per ciò deve considerarsi identica se contenne in sé il cristianesimo che compendiò e superò il pensicro antico.

Tutt'altra questione è se nel Vico sia esplicito e rilevato il concetto di progresso. Il Vico non nega il progresso, vi fa anche, quando parla delle condizioni dei suoi tempi, qualche accenno come a una realtà di fatto; ma non ne ha il concetto, e molto meno gli dà rilievo. La sua filosofia, se procura l'alta visione del processo dello spirito ubbidiente alla sua propria legge, ritiene tuttavia, rla questa mancanza di coscienza circa il progressivo arricchimento del reale, qualcosa di desolato e di triste. Il carattere individuale degli uomini e degli avvenimenti è, nel Vico, obliterato: individui e avvenimenti stanno soltanto come casi particolari di un aspetto dello spirito o di una fase della civiltà; e perciò, sempre, Aristide con Scipione, Alessandro con Cesare, non mai Aristidc come Aristide, Scipione come Scipione, c Alessandro e Cesare come Alessandro e come Ccsare. Progresso importa ufficio privilegiato di ciascun fatto, di ciascun individuo, ciascuno 
mettendo la propria nota, insostituibile, nel poema della storia, e ciascuno rispondente con maggior voce al suo predecessore.

Ma la ragione per la quale al Vico doveva fare difetto l'idea di progresso e la sua ricerca storica doveva riuscire unilaterale, non si può scorgere bene se non quando si sia dato uno sguardo alla sua metafisica. 


\section{XII}

\section{LA METAFISICA}

Per « metafisica » intendiamo la concezione che ebbe il Vico della realta tutta e non del solo mondo umano; e includiamo nel significato della parola anche l'eventuale conclusione negativa che affermi l'inconoscibilità o la imperfetta conoscibilità di una o piú sfere del reale, o di quella suprema in cui le altre si riuniscono.

Il Vico per l'appunto (come ci è noto dalla seconda forma, che è poi l'ultima, della sua gnoseologia) segnò una profonda linea divisoria tra mondo umano e mondo naturale: il primo trasparente all'uomo perché fatto dall'uomo, e il secondo opaco, perché Dio, che l'ha fatto, egli ne ha la scienza. E la sua concezione della realtá totale e ultima, la metafisica da lui esposta tutt'insieme con la sua prima gnoseologia, ritiene il solo valore, che questa le concede, di una probabile ma inverificabile congettura, la quale si compie nella certezza della teologia rivelata. Essa rimane perciò senza possibile congiungimento con la Scienza nuova, che procede con metodo sicuro di verità e prescinde affat to dalla rivelazione. Il Vico non la rifiutó mai; ne discorre nella sua autobiografia che è del 1725, contemporanea alla prima Scienza nuova; la ricorda con compiacimento nel 1737, cioè sette anni dopo la seconda Scienza 
nuova, quando la sua vita scientifica era (ed egli stesso cosí la considerava) terminata. Ma, sebbene non la rifiutasse, la tenne sempre come appartata in un angolo della sua mente.

Sembrerebbe che, assodato questo punto, non ei dovesse essere, circa la metafisica vichiana, altro da dire d'importanza filosofica. Pure, non è cosi. E in primo luogo, poiché ogni parte della filosofia implica le altre e dalla trattazione di una delle cosí dette scienze filosofiche particolari si può sempre desumere il carattere del tutto, è legittimo cercar di determinare, scrutando la Scienza nuova, quale metafisica vi è implicita, ossia quale complemento filosofico quella scienza logicamente sopporta e richiede.

Ora la Scienza nuova, che affermava la conoscibilita piena delle cose umane, e non già nella loro superficie come in una psicologia, ma nell'intima loro natura; la Scienza nuova, che raggiungeva di là dagl'individui la conoscenza della Mente che informa il mondo ed è Provvidenza; quella Scienza, che con divino piacere contemplava l'eterno circolo dello Spirito: innalzata che si era a tale altezza tendeva necessariamente all'interpetrazione di tutta la realtà, della natura e di Dio come Mente. Che questa tendenza fosse oggettiva, della Scienza nuova, e non soggettiva, del Vico, nel quale quella scienza, per cosí dire, si er a pensata, è quasi superfluo avvertire di nuovo. Il Vico, come persona, non solo non la favori, ma anzi la compresse e represse con tanta energia che non ne lasciò apparire traccia nei suoi libri. Di nessuna dottrina filosofica ebbe tanto terrore, e contro nessuna polemizzò con tanta frequenza, quanto contro il panteismo; e forse proprio questa preoccupazione polemica è la sola traccia, sebbene affatto involontaria, che si possa notare nei suoi scritti, della tendenza che egli doveva sentire in sé. Egli 
era e voleva restare cristiano e cattolico: la trascendenza, il Dio personale, la sostanzialità dell'anima, per quanto la sua scienza non vi conducesse, erano bisogni irrefrenabili della sua coscienza. Ma ciò, come permetteva al Vico di reprimere soltanto, e non di sopprimere, la logica e intrinseca tendenza del suo pensiero, cosí dà a noi facoltà di riconoscerla nella cosa stessa. E a ragione un critico italiano (lo Spaventa) ebbe ad affermare che nel Vico si affacci l'esigenza di una nuova metafisica; e un altro, tedesco e cattolico, defini il sistema di lui un semipanteismo. Piú arrischiato sarebbe forse, col ricordato critico italiano, spingersi a dire clee il Vico progredi sul concetto delle due sostanze cartesiane e dei due attributi spinoziani e della stessa monade leibniziana, sorpassando il parallelismo e l'armonia prestabilita col distinguere le due provvidenze, i due attributi, la natura e lo spirito, in modo che uno di essi sia scala all'altro, e col concepire il punto di unione e la derivazione del contrario come spiegamento o sviluppo; onde la natura sarebbe il fenomeno e la base propria dello spirito, il presupposto che lo spirito fa a sé stesso per essere veramente spirito, vera unità. Perché, potendosi dubitare che la distinzione dei due attributi o delle due provvidenze, la naturale e l'umana, sia ben fondata e ineluttabile conseguenza del concepire la sostanza come spirito e come mente, non si può dedurre il passag. gio evolutivo dall'una all'altra come tendenza implicita nel concetto vichiano della mente. Per questa seconda e particolare tendenza occorrono, insomma, prove particolari e documentarie, che si hanno bensí ma insufficienti e malsicure, e non nel sistema della Scienza nuova, ma piuttosto in quello che cronologicamente lo precedette.

Perché anche la metafisica che il Vico delineò nella prina fase del suo pensiero non è (com'è sembrato a parecchi e può sembrare a prima vista) priva di ogni significato e 
importanza. Essa dimostra la medesima avversione contro il materialismo e il medesimo amore per l'idealismo che anima le meditazioni della Scienza nuova. La filosofia di Epicuro, che prende a suo principio il corpo gia formato e diviso in parti multiformi ultime, composte d'altre parti che per difetto di vuoto interposto si fingono indivisibili, sembrara a lui una filosofia da soddisfare le menti rozze dei fanciulli e le deboli delle donnicciuole; e con quanto diletto vedeva spiegate da quel filosofo (ossia nel poema di Lucrezio) le forme della natura corporea, con altrettanto o riso o compatimento lo vedeva tratto dalla dura necessità a perdersi in mille inezie e sciocchezze per ispiegare le guise della mente. Di * falsa posizione», non meno dell'epicurea, il Vico accusava la fisica cartesiana, che anch'essa ha per principio il corpo già formato, diversa da quella di Epicuro in ciò che l'una ferma la divisibilita del corpo negli atomi, l'altra fa $i$ suoi tre elementi divisibili all'infinito; l' una pone il moto nel vano, l'altra nel pieno; l'una comincia a formare $\mathbf{i}$ suoi infiniti mondi da una casuale declinazione di atomi dal moto in giú del proprio loro peso e gravita; l'altra, i suoi indefiniti vortici da un impeto impresso a un pezzo di materia inerte e quindi non divisa ancora, che col moto impresso si divide in quadrelli e impedita dalla sua mole mette in necessita di sforzarsi a movere in moto retto, e, non potendo per il suo pieno, incomincia, divisa nei suoi quadrelli, a moversi circa il centro di ciascun quadrello. Cosí se Epicuro commetteva il mondo al Caso, Cartesio lo assoggettava al Fato; e invano, per salvarsi dal materialismo, egli sovrappose alla sua fisica una metafisica alla maniera platonica, con cui si studiò di stabilire due sostanze, una distesa e l'altra intelligente, e di far luogo a un agente immateriale, perché queste due parti non erano congruenti nel sistema, richiedendo la sua fisica meccanica una metafisica 
come l'epicurea, che stabilisce un sol genere di sostanza corporea opcrante. Per simili o analoghe ragioni, il Vico respingeva le filosofie del Gassendi, dello Spinoza e del Locke; e le fisiche di altri autori, quella per es. di Roberto Boyle, gli parevano profittevoli per la medicina e per la spargirica, inutili per la filosofia. Di Galileo giudicava che avesse mirato la fisica con occhio di gran geometra, ma non con tutto il lume della metafisica. Le sue simpatic si volgevano ai filosofi che erano insieme geometri, e percio alla fisica pitagorica o timaica, secondo la quale il mondo consta di numeri; alla metafisica platonica che dalla forma della nostra mente, senz'alcuna ipotesi, stabilisce per principio di tutte le cose l'idea eterna sulla scienza e coscienza che abbiamo di certe eterne verità che sono nella nostra mente e che non possiamo sconoscere o rinnegare; alla dottrina, che egli attribuiva a Zenone stoico, dei punti metafisici; e, infine, alla filosofia del Rinascimento italiano, quando risplendevano i Ficino, i Pico della Mirandola, gli Steuco, i Nifo, i Mazzoni, i Piccolomini, gli Acquaviva e i Patrizzi.

Il concetto fondamentale della sua cosmologia era dato dai punti metafisici, nei quali trovava applicazione il rioperamento della matematica sulla metafisica, da lui ammesso come procedere analogico costruttivo. Al modo stesso che dal punto geometrico nasee la linea e la superficie, e il punto che viene definito non aver parti da la dimostrazione che le linee altrimenti incommensurabili si tagliano eguali nei loro punti; cosí è lecito postulare punti non piú geometrici ma metafisici, i quali, non estesi, generino l'estensione. Tra Dio, che è quiete, c il corpo, che è moto, s'interpone mediatore il punto metafisico, il cui attributo è il conato, ossia l'indefinita virtú e sforzo dell'universo a mandar fuori e sostenere le cose particolari tutte. L'esistenza del corpo non è altro che un'indefinita virtú di 
mantenerlo disteso, la quale sta egualmente sotto cose distese quantunque disuguali, ed è insieme indefinita virtú di muovere che sta sotto ai moti quanto si voglia disuguali. Sotto un granello di arena vi ha tal cosa che, dividendosi quel corpicello, dà e sostiene un' infinita estensione e grandezza; sicché la mole dell'universo tutto, nel corpo del granello, se non è in atto, è bene in potenza e in virtú. Questo sforzo dell'universo, che è sotto ogni piccolissimo corpicciuolo, non è né l'estensione del corpicciuolo né l'estensione dell' universo; è la mente di Dio, la quale, pura di ogni corpolenzil, agita e muove il tutto. Ogni particolare determinazione della realta si accorda con questa verità fondamentale. Il tempo si divide, l'eternità è nell'indiviso; le perturbazioni dell'animo diminuiscono e crescono, la tranquillita d'animo non conosce gradi; le cosc estese si corrompono, le inestese constano nell'indivisibilità; il corpo tollera divisione, la mente non la tollera; le opportunità sono nel punto, i casi in ogni parte; la scienza non si divide, l'opinione genera le sètte; la virtú non sta né piú in qua né piú in là, il vizio spazia dappertutto; il retto è uno, le cose prave innumerevoli; in ogni genere di cose, insomma, l'ottimo viene collocato nell'indivisibile.

La sostanza in genere, che sta sotto e sostiene le cose, si divide nelle due specie della sostanza distesa, che e quella che sostiene ugualmente estensioni disuguali, e della sostanza cogitante, che sostiene ugualmente pensieri disuguali; e siccome una parte dell'estensione è divisa dall'altra ma indivisa nella sostanza del corpo, cosi una parte della cogitazione, cioè a dire un determinato pensiero, è divisa dall'altra ed è indivisa nella sostanza dell'anima. Proprio dell'anima è il conato, ossia la liberta, negata affatto ai corpi; e Cartesio, che cominciava la sua fisica dal conato dei corpi, la incominciava veramente da poeta e ricadeva nelle concezioni antropomortiche dei popoli primi- 
tivi. Quelli che i meccanici dicono conati, forme, potenze, sono moti insensibili dei corpi, coi quali essi o s'appressano, come voleva la meccanica antica, ai loro centri di gravita, o, secondo le teorie della meccanica nuova, s'allontanano dai loro centri del moto. E, al pari del conato, è inconcepibile nei corpi la comunicazione del moto, concedere la quale tanto varrebbe quanto concedere la compenetrazione dei corpi, non essendo altro il moto che il corpo che si muove: la percossa data a una palla è soltanto occasione perché lo sforzo dell' univer'so, il quale era sí debole nella palla da far sembrare che essa si mantenesse quieta, si spieghi di piú e cosí ei dia apparenza di piú sensibile moto. Coi cartesiani, per altro, e in ispecie col Malebranche, il Vico s'accordava circa l'origine delle idee, inclinando alla concezione che Dio le crei in noi volta per volta; coi cartesiani altresi teneva che i bruti fossero macchine; e con tutta la filosofia del suo tempo riconosceva la soggettivita delle qualitì sensibili.

Lascianclo queste ultime dottrine, alle quali il Vico accenna appena e che non gli sono proprie, tutta sua veramente è quella fondamentale dei punti metafisici; giacché l'attribuzione di essa a un fantastico Zenone, nella cui persona erano fusi e confusi l'eleate e lo stoico (secondo un errore comune nella letteratura filosofica del tempo), non può ingannare nessuno, e non ingannò il medesimo Vico clıe, messo alle strette, spiegò come fosse stato condotto a interpetrare a quel modo ciò che di Zenone riferisce Aristotele e concluse che, se quella dottrina non si voleva ricevere come zenoniana, la si prendesse per sua propria e non assistita da nomi grandi. Né, d'altro canto, si può riportarla alla monadologia leibniziana, che è dubbio se fosse nota al Vico, che il Vico a ogni modo non mentova (laddove pur mentova, con parole di alta reverenza, il Leibniz), e con la quale la somiglianza è molto 
vaga, perché i punti metafisici non sono monadi. Se mai, qualche efficacia si puỏ affermare che avesse sopra di essa la scoperta leibniziana e newtoniana (che allora si cominciava a divulgare in Italia anche per opera di taluni amici personali del Vico) del calcolo infinitesimale; i cui termini d'infiniti massimi, minori, maggiori e via dicendo (egli dice) stravolgerebbero l'umano intendimento, perché l'infinito è schivo di ogni moltiplicazione e comparazione, se non soccorresse una metafisica la quale stabilisca che sotto tutti gli attuali distesi e attuali movimenti sia una virtú o potenza di estensione e di moto sempre uguale a sé stessa, cioè infinita. E piú giustamente ancora è stato indagato il confluire nella concezione vichiana delle correnti platoniche (del platonismo della Rinascenza) e galileiane, particolarmente di queste ultime; il che, per altro, non ne diminuisce l'originalità.

Originalita, senza dubbio, di un pensare fantasticheggiante e arbitrario, che per tal ragione rimase senza possibilita di svolgimento e senza efficacia diretta sulla restante concezione del Vico. Al recensente del Giornale dei letterati, che chiamava quella metafisica un abbozzo, l'autore rispondeva che era affatto compiuta: un aborto, invero, piuttosto che un abbozzo, e, come aborto, compiuto. E nella Scienza nuova, oltre qualche richiamo alla negata attribuzione del conato ai corpi, c'è un solo fuggevole ma curioso tentativo di connessione con una metafisica geometrica o aritmetica sul tipo di quella ora delineata; ed è là dove si afferma che sull'ordine delle cose civili corpulente e composte si conviene l'ordine dei numeri, che sono cose astratte e purissime, e si osserva che, infatti, i governi cominciano dall'uno con le monarchie familiari, passano ai pochi con le aristocrazie, s'inoltrano ai molti e tutti nelle repubbliche democratiche, e finalmente ritornano all' uno nelle monarchie civili (assolute), sicché l'umanità 
corre sempre dall'uno all'uno, dall'assolutismo del paterfamilias a quello del monarca illuminato.

Ma se si può e si deve negar valore alla cosmologia vichiana; se le contradizioni e le oscurita in cui si avvolge sono manifeste e furono notate dai critici del tempo; è anche innegabile il carattere che essa ha di dinamismo, in opposizione al meccanicismo della filosofia contemporanea. L'escogitazione dei punti metafisici, nella quale Dio appare il gran geometra che conoscendo fa e facendo conosce le cose dell'universo, è come il simbolo della necessità di risolvere la natura in termini idealistici. Un Vico teologizzante, un Vico agnostico, perfino un Vico immaginoso inventore di romanzi cosmologici e fisici, si trova qua e là; ma un Vico materialista non si trova in nessuna parte dell'opera sua.

Anche questa non ardita metafisica destò sospetto di panteisino, benché l'autore insistesse nella dottrina teologica che il fire di Dio si converte $\alpha a$ intra "col generato $\mathrm{e}$ « $a b$ extra» col fatto, e che perciò il mondo è creato in tempo; che l'anima nmana, la quale, specchio della divina, pensa l'infinito e l'eterno, non è terminata da corpo e quindi neppure da tempo, e perciò è immortale; e che in qual modo l'infinito sia disceso nelle cose tinite, ciò, se anche Dio l'insegnasse, non si potrebbe intendere dall'uomo. Comunque, egli stimò necessario chiudere le risposte ai suoi critici col raccogliere le proposizioni che dimostravano il suo ortodossismo, e ribadire che « essendo Dio altrimente sostanza e altrimente le creature, e la ragion d'essere o l'essenza essendo propria della sostanza, le sostanze create, anche in quanto all'essenza, sono diverse e distinte dalla sostanza di Dio ».

La trascendenza limitava la mente del Vico e, impedendogli di raggiungere l'unità del reale, gl'impediva anche la conoscenza veramente completa di quel mondo 
umano, ch'egli aveva cosí potentemente, con opposto principio, rischiarato. Ed ecco ora perché il Vico, senza negare il progresso, non poteva averne il concetto. $\dot{E}$ stato osservato che il concetto di progresso è estraneo al cattolicismo e prende origine dalla riforma protestante, e che percio il cattolico Vico doveva inibirselo. Ma altresi il concetto della provvidenza immanente è inconciliabile col cattolicismo, e tuttavia il Vico lo pensò profondamente. Il che vuol dire che non l'impulso gli mancava, ma piuttosto la possibilità di andar oltre un certo segno, dove la sua fede sarebbe stata messa a troppo aperto sbaraglio. Il progresso, dedotto dalla provvidenza immanente e introdotto nella Scienza nuova, avrebbe accentuato la differenza nell' uniformità, il sorgere del nuovo a ogni istante, il perpetuo arricchimento del corso a ogni ricorso; avrebbe cangiato la storia, da un rassegnato percorrere e ripercorrere il solco tracciato da Dio sotto l'occhio di Dio, in un dramma che ha in sé la propria ragion d'essere; avrebbe trascinato nelle sue spire l'intero cosmo e reso reale il pensiero dei mondi infiniti. Il Vico, all'affacciarsi di questa visione, arretra pauroso, si ferma ostinato, e il filosofo è sostituito in lui dal credente. 


\section{XIII}

\section{Passaggio alla storiografia}

Carattere generale della storiografia vichiana

D

alle cose precedentemente discorse è chiaro che la parte storica della Scienza nuova non poteva configurarsi come una storia del genere umano, nella quale ai popoli e agl'individui fosse riconosciuto l'ufficio proprio e singolare che ciascuno di essi esercitò nel corso degli avvenimenti. A tal uopo il Vico avrebbe dovuto chiudere il suo sistema di pensiero, che in un punto rimaneva spezzato e aperto alla concezione religiosa; e innalzare la sua divinita provvidente a divinita progrediente, determinando i corsi e i ricorsi come il ritmo interno del progresso. Ovvero, per raggiungere nella storia, in senso diametralmente opposto, la visione dell' individualità, doveva abbandonare la sua germinale filosofia idealistica, togliere la divisione tra provvidenza ordinaria e straordinaria, darsi totalmente in braccio alla fede e alla tradizione religiosa, e tracciare la storia dell' umanità sul disegno che Dio aveva rivelato o permetteva d'intravvederc. Come credente, egli repugnava al primo partito, come filosofo, al secondo; onde la storia da lui ricostruita non poteva essere, e non fu, storia universale.

Per conseguenza, non fu neppure quello che si chiama filosofia della storia, se a questa denominazione si rida 
il significato originario di una "storia universale " (cioè che abbia l'occhio alle maggiori e piú nascoste iuncturce rerum) « narrata filosoficamente (vale a dire, piú filosoficamente che non si solesse dai cronisti, dagli aneddotisti e dagli storiografi cortigiani, politici e nazionali). La controversia se al Vico o allo Herder spetti di aver fondato la filosofia della storia, dovrebbe francamente risolversi a favore dello Herder, perché l'opera di costui ha quell'andamento di «storia universale » che manca alla Scienza nuova; sebbene, d'altro canto, sia agevole trovare allo Herder precursori in buon numero, a cominciare dai profeti ebraici e dallo schema delle quattro monarchie, che rimase non solo nel Medioevo ma ben oltre nei tempi moderni lo schema costruttivo della storia universale. Né sarà fuori luogo soggiungere che la cosi detta filosofia della storia in quanto storia universale non costituisce una speciale scienza filosofica o una storia nettamente distinguibile da altre forme di storia (salvo che, per ismania di renderla autonoma, non se ne faccia il mostro di una storia astratta o di una filosofia storicizzata); e quando al Vico o allo Herder si attribuisce il vanto di avere creato con la filosofia della storia una nuova scienza, si rivolge loro un complimento di dubbia lega: il quale, per ciò che in particolare concerne il Vico, è stato cagione che non si scorgesse il valore vero dall'opera sua. Infatti, la « Scienza nuova d'intorno alla comune natura delle nazioni », intesa come l'equivoca seienza della filosofia della storia (Phi'losophie de l'histoire intitolò il Michelet la sua riduzione francese dell'opera vichiana), non ha lasciato vedere la Scienza nuova come nuova filosofia dello spirito e iniziale metafisica della mente.

Il dissidio che era, nella sua concezione generale, tra scienza e credenza, riappare, nella storiografia del Vico, come divisione e opposizione tra storia degli ebrei e 
storia delle genti, tra storia sacra e storia profana. La storia ebraica non andò soggetta alle leggi delle altre, ebbe un corso tutto proprio, si spiega con principî affatto particolari, cioè con l'azione diretta di Dio. La Scienza nuova, che nella sua parte filosofica non ne dava i principî esplicativi, non avrebbe dunque dovuto trattarne altrimenti nella sua parte storica. E tale sarebbe stato, forse, il desiderio del Vico. Ma al desiderio si opponeva (senza parlare del bisogno in cui egli era di premunirsi della taccia di empieta, che non sarebbe mancata) il suo scrupolo di nomo di fede e di buona fede, che lo spingeva a cercare una qualche armonia tra le due storie, le quali, per quanto divise egli le ponesse (ricordando in proposito che anche un autore gentile, Tacito, chiamava gli ebrei « uomini insocievoli »), entrambe si erano svolte sulla terra e avevano avuto reciproche relazioni, non foss'altro che all'origine dell'umanita e nella sua palingenesi per opera del cristianesimo. Accadde che il Vico il quale voleva e doveva, per l'indirizzo stesso della sua mente, evitare il racconto della storia universale, e attenersi insieme ai soli problemi filosoficamente e filologicamente trattabili, non potesse esimersi dal rompere talvolta il suo proposito, e dal tentare un qualche congiungimento tra le due storic, e in pari tempo una qualche apologia della storia sacra con gli argomenti forniti dalla scienza e dalla storia profana.

È questa la parte piú infelice ma altamente significante dell'opera sua. Egli era costretto ad ammettere, in contrasto a tutte le sue scoperte, con istrazio di tutta la sua mente, che gli ebrei avevano goduto il privilegio di serbare intatte le loro memorie fino dal principio del mondo, della qual cosa le altre nazioni si vantavano a vuoto, e che perciò l'origine e successione certa della storia universale dovesse domandarsi alla storia sacra. E l'esigenza di connettere i suoi concetti circa le civiltà primitive con 
la cronologia biblica, con l'anno che si soleva assegnare alla creazione del mondo, con la tradizione del diluvio - universale e con quella dei giganti, di trovare (com'egli dice) « la perpetuità della storia sacra con la profana , lo portò a immaginare le cose piú stravaganti. Imperversato dunque nell'anno 1656 dalla creazione il diluvio, o separatisi i figli di Noè, mentre gli ebrei iniziano o proseguono la loro sacra storia con Abramo e gli altri patriarchi, e poi con le leggi date da Dio a Mosè, tutti i restanti semiti e i camiti e giapetici, i primi piú tardi e per minor tempo, i secondi e i terzi piú presto e per tempo piú lungo, caddero nello stato ferino ed errarono per la terra, bestioni stupidi e feroci. E laddove gli ebrei, sottomessi al governo teocratico, severamente educati e praticanti le abluzioni, rimasero di giusta statura, i componenti delle altre razze, senza disciplina né morale né fisica, travolgendosi nel fango, nello sterco e nell'urina e assorbendo sali nitrici (eosi cone di sterco e di urina la terra s'ingrassa e diventa feconda) crebbero in corpi mostruosi e giganteschi. Cento anni pei semiti e dugento per le altre due razze durò lo stato ferino; fino a quando la terra, che era rimasta a lungo inzuppata dall'umidore del diluvio universale, asciugandosi mandò fuori esalazioni secche o materie ignite in aria a ingenerare $i$ fulmini. Coi fulmini, come gia sappiamo, e con la mitologia del cielo fulminante che è Giove, si sveglia nei bestioni la coscienza di Dio e la coscienza di sé, onde diventano uomini. Si apre cosí l'età degli dèi, che socialmente è quella delle monarchie familiari dove il padre è re e sacerdote e nel corso della quale si viene costituendo il sistema delle deità maggiori, e i giganti mercé le spaventose religioni e l'educazione domestica che doma la loro carne e sviluppa in essi l'elemento spirituale, e mercé le lavande, degradano via via alla giusta corporatura quale hanno gli uomini che s'in- 
contrano agli inizî della susseguente età eroica. - Tale, indicata per sommi capi, è la bizzarra costruzione, fatta dal Vico, dei cominciamenti della storia umana sulla terra, messi in armonia coi racconti della storia sacra; e di essa si sarebbe l'iso o sorriso meno, se si fosse guardato al dramma che vi è sotto, alla tormentosa coscienza del eredente che, lottando col pensatore, cerca rifugio in quelle stravaganze. Con le quali, a ogni modo, il Vico valicava sopra una serie di sassi vacillanti (il diluvio, i giganti, le esalazioni secche) la fiumana della tradizione religiosa e raggiungeva il terreno sodo della storia critica, dove scopriva altresí il primo appoggio della sua filosofia dello spirito, la ferinità. È da osservare inoltre che il rapporto con la storia ebraica - la sola che a lui s'imponesse come storia vera e propria, cioè come un unicum, sebbene in modo miracoloso, affatto individuato - gli suggeri i rari accenui che s'incontrano nei suoi scritti ad assegnare ai varî popoli uno speciale ufficio o missione; onde gli parve talvolta che gli ebrei rappresentassero la mens, i caldei la ratio e i giapetici la phantasia.

Parallelamente a questa storia fantastica dei cominciamenti del gellere umano sulla terra, corrono i tentativi di apologetica biblica. Il Vico non tralascia di arrecare prove che dovrebbero profanamente confermare i racconti della storia sacra. Conferma, per es., del diluvio e dei giganti sarebbero i simiglianti racconti dei greci e di altri popoli; il governo teocratico, del quale nessuna storia profana ha notizia precisa e oscuramente vi alluclono i poeti nelle loro favole, si riscontrerebbe nel governo degli ebrei innanzi e dopo il diluvio; - gli ebrei avrebbero ignorato la divinazione, perché vivevano in diretti rapporti col vero Dio, laddove i caldei ebbero la magia o divinazione secondo i moti degli astri e i popoli di Europa quella per auspicî. Si sente in tutto ciò, senza dubbio, qualcosa di voluto, un 
voler vedere o un voler non vedere, un darsi sulla voce, un eccitarsi alla persuasione; come è consueto, del resto, in molti credenti colti e scientificamente educati. Il Vico scriverà perfino una volta, nell'esporre la genesi storica delle forme grammaticali e nell'asserire che i verbi cominciarono dagli imperativi e cioc̀ dai comandi monosillabici che i padri davano a mogli, figliuoli c famoli (es, sta, $i, d a$, fac ecc.), che da ciò si ricava un'indiretta dimostrazione della verità del cristianesimo, perché in ebraico la terza persona singola e maschile del perfetto è rappresentata dalla nuda radice senza alcun segno flessivo: prova evidente che i patriarchi dovettero dare gli ordini nelle loro famiglie a nome di un sol Dio (Deus dixit). Questo, a suo parero, era « un fulmine da atterrare tutti gli scrittori che hanno oppinato gli ebrei essere stati una colonia uscita da Egitto, quando, dall'incominciar a formarsi, la lingua ebraica ebbe incominciamento da un solo Dio ». Sono fulmini, a dir vero, che invece di fulminare i miscredenti, illuminano la povertà degli argomenti sui quali l'apologetica si appoggia anche in un uomo come il Vico; e, oggettivamente considerando, la divisione introdotta per iscrupolo religioso tra storia sacra e storia profina, col conseguente trattamento eritico di questa e dommatico di quella, e con le conseguenti strane ipotesi e difese, faceva e fa pensare irresistibilmente che il sottrarsi della storia sacra alla scienza umana provenga non dall'impotenza della scienza umana, ma dall'impotenza della storia sacra, cioè, dall'impotenza a serbarsi inalterata nella scienza; sicché di rado uno scrupolo religioso $\mathrm{fu}$ di tanto pericolo alla causa della religione.

Ma il Vico aveva troppo genuino e rigoroso senso scientifico da mettersi a fare, e per giunta a contraggenio, il Selden o il Bossuet; onde l'armonizzamento con la storia sacra o l'apologetica rimangono in lưi episodî, dai quali 
si può prescindere. E poiché, d'altra parte, gli era vietato di profanare del tutto la filosofia e la storia, e di rappresentare il movimento storico complessivo in base al criterio del progresso, non gli restava se non guardare i fatti dall'aspetto che la sua filosofia gli coneedeva libero: quello dei corsi e ricorsi, dell'eterno processo e delle eterne fasi dello spirito. Qui era la sua forza, qui poteva riconoscere il carattere specifico, se non propriamente quello individuale, di leggi, costumi, poesie, favole, d'intere formazioni sociali e culturali che erano state fraintese dalla storiografia fino ai suoi tempi. E per questa ragione egli, anziché narrare la storia, doveva restringersi a mettere in luce gli aspetti comuni di certi gruppi di fatti, appartenenti a tempi e nazioni varie. Nella Scienza nuova si ha (egli dice) - tutta spiegata la storia, non gi à particolare ed in tempo delle leggi e dei fatti de' romanie de'greci, ma sull'identita in sostanza d'intendere e diversita dei modi lor di spiegarsi». * Si arrecheranno (dice ancora in altra occasione) i fatti a modo di esempli perché s'intendano in ragion di principî », imperocché «vedere avverati i principî nella quasi innumerabile folla delle conseguenze, egli si dee aspettare da altre opere che da noi o gia se ne son date fuori o gia sono alla mano per uscire alla luce delle stampe ». Ossia, come sappiamo, in quella Scienza si ha da una parte una filosofia e dall'altra una descrittiva empirica, storicamente esemplificata, nella quale i romani non stanno come romani, ma in ciò che hanno di comune coi greci o magari coi giapponesi; la storia di Roma sotto i re o ai primi tempi della repubblica spiega le sue affinità con quella dei primi secoli del Medioevo; e Omero non sta come Omero, ma come esempio della poesia primitiva e, attraverso i secoli, ritrova e abbraccia il suo fratello, Dante. Forza e limite insieme, perché la storia non consiste di certo, essen- 
zialmente, in queste somiglianze; ma senza la percezione delle somiglianze come si giungerebbe a fissare le differenze? Dante non è Omero, i baroni non sono $\mathrm{i}$ « patres », l'ateniese Solone non è il romano Publilio Filone, il feudalismo dell'età carolingia e in genere medievale non è la costituzione sociale delle eta primitive di Grecia c di Roma; ma certamente, per taluni rispetti, Dante è piú vicino a Omero che non al Petrarca, i baroni della prima epoca piú prossimi ai « patres» che non alla posteriore nobilta di corte, Solone somiglia piú a un tribuno o a un dittatore romano che a qualche altro dei sette savî coi quali suole andare congiunto, il feudalismo medievale si rischiara col ravvicinamento alle societa fondate sull'economia agraria. Notare queste somiglianze significa negare o rigettare indietro altre più superficiali e aprire la via alla conoscenza dell'individualità, indicando la regione approssimativa dove si trova la verita piena. Il Vico, piuttosto che narrare e rappresentare, classifica; ma c'è classificazione e classificazione: quella che si fa a servigio di un pensiero superficiale e quella che si fa a servigio di un pensiero profondo. E la parte storica della Scienza nuova è una grande sostituzione di classificazioni superficiali con classificazioni profonde.

In questo àmbito, dov'è la forza della storiografia vichiana, le deficienze e gli errori provengono non dal di fuori dei limiti tracciati, ma da cagioni operanti dentro quei limiti stessi. É stato allegato, in discolpa del Vico, che gran parte dei suoi errori sono da attribuire ai materiali scarsi e insufficienti dei quali egli disponeva; ma scarsi e insufficienti rispetto alla nostra brama di sapere sono, sempre, i materiali di studio, e nel giudicare uno storico non può essere questione di ciò, sí del modo cauto o incauto nel quale egli adopera i materiali di cui dispone. Ancora è stato detto che il Vico ebbe i difetti del suo tempo; 
e qui si è dimenticato che egli nasceva nel secolo nel quale si era svolta la criticissima filologia di Giuseppe Scaligero e di tutta la scuola olandese, e che suoi contemporanei furono in Italia lo Zeno, il Maffei e il Muratori. Il vero è che la forma mentale da noi già descritta, del Vico, come turbava la pura trattazione filosofica con le determinazioni della scienza empirica e dei dati storici, cosí turbava la ricerca storica col miscuglio della filosofia e della scienza empirica. Il Vico era in uno stato come di ebrezza: confondendo categorie e fatti, si sentiva molto spesso sicuro a priori di quel che i fatti gli avrebbero detto e non li lasciava parlare e subito metteva loro in bocca la sua risposta. Una frequente illusione gli faceva ravvisare rapporti tra cose che non ne avevano alcuno; gli inutava ogni ipotetica combinazione in certezza; gli faceva leggere negli autori, invece delle parole esistenti, altre non mai scritte e ch'egli medesimo senz'accorgersene aveva interiormente pronunziate e proiettate negli scritti altrui. L'esattezza gli era impossibile, e in quella sua eccitazione ed esaltazione di spirito, quasi la disprezzava; perché, infatti, dieci, venti, cento errori particolari che cosa avrebbero tolto alla verità sostanziale? L'esattezza, la « diligenza » (egli dice) « dee perdersi nel larorare d'intorno ad argomenti c'hanno della grandezza, perocché ella è una minuta e, perché minuta, anco tarda virtú ». Etimologie immaginose, interpetrazioni mitologiche arrischiate e infondate, scambî di nomi e tempi, esagerazioni di fatti, citazioni fallaci s'incontrano a ogni passo nelle sue pagine e molte sc ne possono vedere notate nella bella edizione della seconda Scienza nuova, curata dal Nicolini, e qualcuna ne noterenıo via via anche noi a mo' di saggio, na guardandoci dal dirgli di continuo sulla voce, e qualche volta rettificando tacitamente le sue citazioni. Sicché, come parlando della sua filosofia abbiamo osservato che il 
Vico non era ingegno a cuto, cosí, parlando della sua storiografia, dobbiamo ora dire che egli non era ingegno eritico. Ma come, negandogli cola l'acume in piccolo, gli riconoscevamo quell'acume in grande che è la profondità, cosí anche qui dobbiamo aggiungere che, se il Vico mancava di senso critico in piccolo, abbondava di quello in grande. Negligente, cervellotico, affastellato nei particolari; circospetto, logico, penetrativo nei punti essenziali; scopre il fianco, e talora tutta la persona ai colpi del piú meschino e meccanico erudito, e intimidisce ed è atto a ispirare reverenza a ogni critico e storico, per grande che sia. E se spaziando sempre negli universali e tutto preso dalle sue geniali scoperte, molte volte non dié tempo àl tempo e non dié agio e campo alla sua forza indagatrice e osservatrice di spiegarsi, e invece di storia inventò miti e intessé romanzi; dove poi lasciò che quella forza liberamente si spiegasse, compi anche nel campo. della storia cose mirabili, come e'industrieremo di venire mostrando nei capitoli che seguono.

Ma passare a rassegna le interpetrazioni storiche vichiane per confrontarle, come da molti si è fatto ed è comune vezzo, con quelle della storiografia odierna e lodarle o censurarle di conseguenza, sarebbe poco concludente; perché, dove c'è accordo tra i due termini del confronto, l'accordo potrebbe essere fortuito, e, dove e'è divergenza, la dottrina recente potrebbe essere pur tuttavia svolgimento o conseguenza del tentativo antico, e, a ogni modo, lo stato odierno delle cognizioni storiche non porge in niun easo una misura assoluta. E, d'altra parte, sarebbe fuori luogo (oltreehé superiore alle nostre forze) ripigliare tutti i problemi che il Vico trattò e toccò per esaminare quel che di vero o di falso fosse nelle sue conclusioni, perché tanto varrebbe scrivere una terza Scienza nuova, meglio conforme ai nostri tempi. A noi spetta indicare soltanto i principali pro- 
Xi1I. CARATTERE DELla Storiografia VICHIANA 159

blemi storici che egli si propose, riassumere le soluzioni che ne diede, e avere l'occhio sempre allo stato della scienza non già ai tempi nostri ma ai tempi suoi, per determinare quali progressi si debbano al Vico nclla storia degli studî storici. 
$$
\text { - }
$$

\section{.}




\section{XIV}

\section{NuOVl CANONI}

PER LA STORIA DEL TEMPO OSCURO E FAVOLOSO

$\mathrm{T}_{1}$

periodo storiografico, che precedette il Vico, fu, come s'è detto, tutt'altro che di credulità e di acrisía. Trascorsi da un pezzo erano i tempi in cui si compilavano le * cronache del mondo * si accoglieva ogni favola e ogni piú grossolana falsificazione come storia: i semi sparsi da alcuni umanisti avevano portato i loro frutti negli cruditi italiani, nella scuola giuridica francese, nella gia ricordata scaligeriana, in tutti i grandi cronologi, epigrafisti, archeologi, topografi e geografi, che ordinarono nel secolo decimosettimo le prime e colossali raccolte critiche di fonti per la storia dell'antichità. Anzi, nel tempo stesso che i filologi andavano correggendo e perfezionando i loro metodi e sfatavano imposture e riempirano lacune, si diffondeva, per effetto della filosofia intellettualistica, lo scetticismo, o pirronismo storico come anche era chiamato, col Bayle, col Fontenelle, col Saint-Evremond e altri molti, plecursori di quella polemica contro la verita c l'utilita della storia, che doveva diventare cosi vivace nel secolo seguente.

Quest'ultimo indirizzo era, piuttosto che eritico, ipercritico, mettendo capo alla distruzione della storia in ge- 
nere; e poiché lo scetticismo storico rivestí assai spesso il carattere di paradosso a uso della societa elegante e dei belli spiriti, la sua efficacia sul progresso degli studî fu assai scarsa, o, tutt'al piú, valse a provocare vigorose reazioni (di una delle quali fu rappresentante il Vieo) a farore della tradizione e dell'autorità. Giova invece notare le defieienze del primo e seriamente scientifico indirizzo dei filologi e antiquarî: i quali restituivano testi, svelavano falsifieazioni, ricostruivano serie di sovrani e di magistrati, raddrizzavano la cronologia, contestavano perfino alcune leggende; ma, sia per la mentalita consueta dei puri eruditi e filologi, sia per l'ambiẹte generale della cultura di quel secolo, pur vivendo sempre a contatto dell'antico e del primitivo, non sentivano punto, e non facevano sentire, l'antico e il primitivo. Fortissimi nei particolari, erano deboli nelle cose essenziali. Anche quando aleuno dei piú geniali si accorgeva, per es., dell'importanza dei canti popolari, mezzo di trasmissione storiea in tempi in eui mancava o era rarissimo l'uso della serittura, da queste e simili osservazioni non riceveva tale scossa da esserne spinto a rinnovare da cima a fondo la sua coneezione della vita primitiva, come aecadde invece al Vico, il quale, quasi a un tempo, intese la forma filosofiea del certo e i due periodi di vita spirituale e sociale, che le corrispondevano nella storia reale: il periodo oscuro e quello favoloso.

Anch'egli moveva da una sorta di scetticismo, seetticismo concernente i pregiudizî dei dotti e delle nazioni circa l'indole e i fatti dell'antichita; e statuiva, nel combatterli, una serie di canoni o "degnita », che paiono ispirati agli « idola del Bacone, di cui offrono come l'analogo nel campo della ricerca storica. Il Vico metteva in guardia in primo luogo contro le magnifiche opinioni ehe si erano avute fino ai suoi tempi intorno alla lontanissima e sconosciuta antichità: ingenua illusione di cui 
trovava la sorgente in ciò che l'uomo, allorché si rovescia nell'ignoranza, fa di sé regola dell'univer'so (e qui è piú vicina l'analogia col Bacone, perché tale enunciato somiglia per l'appunto alla classe degli « idola tribus» in cui la mente fa di sé regola delle cose, ex analogia hominis, non ex analogia universi). Sopra la medesima osservazione si fonda il detto che "fama crescit eundo », e il tacitiano: " omne ignotum pro magnifico est ». Donde il vezzo d' interpetrare i costumi antichi con l'aspettazione di trovarli simili o migliori di quelli moderni e civili. Cosí Cicerone, per « un trasporto di fantasia » ammirava la mansuetudine degli antichi romani, che chiamavano « ospite 》 il nemico di gucrra; non avvedendosi che la cosa stava proprio al rovescio e che gli ospiti erano « hostes », stranieri e nemici. Parimente Seneca, per provare che convenga usare umanita verso gli schiavi, ricordava che i padroni erano detti in antico * padri di famiglia $»$ quasi che $i$ «patresfamilias » non fossero stati disumanissimi, nonché contro gli schiavi e famoli, contro i medesimi loro figliuoli, adeguati ai famoli. E per lo stesso pregiudizio il Grozio (che veramente il Vico scambia qui col suo esegeta Gronovio e di costui fraintende le parole), volendo dimostrare la mitezza degli antichi germani, recava un gran numero di leggi barbariche, nelle quali l'omicidio era punito con la multa di pochi danari: documento, per contrario, di quanto fosse tenuto a vile il sangue dei poveri vassalli rustici, che erano per l'appunto gli « homines », di cui parlavano quelle leggi.

In secondo luogo, ammoniva di non prestare fede alla boria delle nazioni, che (come avrebbe osservato Diodoro siculo) tutte sia greche sia barbare - caldei, sciti, egizî, cinesi - si vantarono di avere, ciascuna prima delle altre, fondata l'umanità, ritrovati i comodi della vita e serbate le loro memorie fin dalle origini del mondo. Ciascuna 
di esse, non avendo per molte migliaia d'anni avuto commercio con le altre onde potesse accomunare le notizie, $\mathrm{fu}$, nel buio della sua cronologia, simile a un uomo che, dormendo in una stanza piccolissima, nell'errore delle tenebre la crede certamente molto maggiore di quanto con le mani la toccherà poi. Chi prenda quei sognati vanti per notizie sicure, si trova nell'imbarazzo di scegliere fra tante nazioni e tante memorie, tutte, con pari fondamento, offrentisi a gara come primitive.

Con la boria delle nazioni il Vico metteva la boria dei dotti, i quali ciò che essi sanno vogliono che sia antico quanto il mondo; e percio si compiacciono nell'immaginare una inarrivabile riposta sapienza degli antichi, che coincide poi per l'appunto, mirabilmente, con le opinioni professate da ciascuno di quei dotti e da essi ammantate di antichita per imporne piú solennemente l'accettazione. In tale errore cadde non solo Platone, specialmente nelle ricerche del Cratilo, ma quasi tutti gli storici, antichi e moderni: vi era caduto lo stesso Vico (che poté, dunque, studiarlo assai bene in sé medesimo), quando nel De antiquissima aveva creduto di trovare nelle etimologie dei rocaboli latini le prove di una metafisica italiana perfettamente concorde con quella sua propria della conversione tra «verum e « factum e dei punti metafisici.

Ai quali tre pregiudizî, e piú strettamente alla boria dei dotti, va di séguito il quarto che ora si chiamerebbe delle «fonti » o degli «influssi di cultura », e che il Vico sarcasticamente designava come quello della successione delle scuole per le nazioni. Secondo tale dottrina, Zoroastro, per es., avrebbe istruito Beroso per la Caldea, Beroso a sua volta Mercurio 'Trismegisto per l'Egitto, Mereurio Atlante legislatore dell'Etiopia, Atlante Orfeo missionario della Tracia, e finalmente Orfeo avrebbe fermato la.sua scuola in Grecia. Lunghi viaggi, e agevoli, 
in verità, a quelle prime nazioni che, appena uscite dallo stato selvaggio, vivevano appollaiate sulle montagne in siti poco accessibili, sconosciute alle loro medesime confinanti! E questi lunghi viaggi avrebbero avuto per oggetto di diffondere invenzioni, che ciascuna nazione poteva fare senz'altro da sé, e che se poi, conosciutisi tra loro i popoli per guerre e trattati, si ritrovarono simili, fu perché contenevano un motivo comune di vero e nascevano dalle medesime necessità umane. C'era bisogno di supporre l'efficacia del diritto ateniese o di quello mosaico sul romano, come usavano $\mathrm{i}$ «pareggiatori » delle leggi o trattatisti del diritto comparato, per ispiegare come si fosse formato il diritto, riconosciuto in Palestina, in Atene e in Roma, di uccidere il ladro di notte? C'era bisogno che Pitagora andasse diffondendo la dottrina della trasmigrazione delle anime, che si ritrova perfino in India?

Restava il pregiudizio circa gli storici antichi conside. rati come informatissimi dei tempi primitivi, i quali, invece, nel racconto delle origini, seppero quanto o meno di noi posteri. Per la storia greca, il Vico leggeva, o meglio credeva di leggere, in Tucidide la confessione che i greci, fino alla generazione a questo storico precedente, non conoscevano nulla della propria antichità; c osservava altresí che gli storici greci solo al tempo di Senofonte cominciarono ad avere qualche notizia precisa delle cose persiane. La storia romana si soleva principiarla da Roma; ma con Roma certamente non nacque il mondo, la quale fu una cittả nuova fondata in mezzo a un gran numero di minuti popoli piú antichi nel Lazio; e per Roma stessa Tito Livio dichiara di non entrare mallevadore della verità dei fatti concernenti i primi secoli di quella storia, e a proposito della seconda guerra cartaginese, di cui è in grado di scrivere con piú verità, ingenuamente confessa di non sapere da qual parte Annibale fece il suo 
grande e memorabile passaggio in Italia, se dalle Alpi cozie o dalle appennine. Tanto gli storici antichi erano bene informati!

Per questi e altrettali motivi di scetticismo, tutto quanto si narrava dei greci fino al tempo di Erodoto e dei romani fino alla seconda guerra cartaginese parve al Vico tutto incertissimo: un territorio quasi res nullius, ove si poteva entrare col diritto del primo occupante. Egli vi entrava armato dei canoni positivi che nascevano accanto, anzi dal grembo di quelli negativi, che abbiamo riferiti. Perché, se il Vico negava fede agli storici lontani dai tempi e luoghi dei fatti che raccontavano, se screclitava le vanterie nazionali, se svelava le illusioni e le ciarlatanerie dei dotti, noll rimaneva pago per altro a quest'opera di distruzione; e al posto del vecchio e malfido cacciato via badava a sostituire il nuovo di migliore qualità e di maggiore resistenza, cioè un complesso di metodi mercé i quali era dato procacciarsi nuovi documenti con lo studiare meglio quelli già posseduti. Ogni avanzamento nelle conoscenze storiche non si effettua, in veriti, in altra guisa che con questo ritorno dal racconto ricevuto al documento sottostante, col quale solamente è dato confermare, rettificare e arricchire il racconto.

Il primo metodo che il Vico addita, la prima fonte che egli schiude per la conoscenza delle societa antichissime, è l'etimologia delle lingue, che si soleva esercitare ai suoi tempi in modo affatto arbitrario, col raffrontare $i$ suoni di qualche sillaba o lettera, e cercare altre superficiali somiglianze, inferendone la derivazione di un vocabolo da una lingua o dall'altra, dal latino, dal greco o dall'ebraico. Ma affinché l'etimologizzare sia fiuttuoso, bisogna non dimenticare che le lingue sono i testimoni piúgravi degli antichi costumi dei popoli, che si celebrarono al tempo in cui si formarono esse lingue; e illuminare per- 
cio, perpetuamente, le lingue coi costumi e i costumi con le lingue. Cosí le etimologie dei vocaboli astratti ci portano nel bel mezzo di una società affatto contadinesca, perché l' « intelligere», per es., richiama il * legere » o raccogliere i frutti dei campi (donde «legumina»); il « disserere », lo spargere semenze; e la maggior parte delle espressioni intorno a cose inanimate si svelano trasporti dal coipo umano e dalle sue parti e dagli umani sensi e passioni, come * bocca » per ogni apertura, « labbro per orlo di vaso, * fronte e spalle » per avanti e clietro, e simili. Il Vico vagheggio un etimologico comune a tutte le lingue native, composto di radici monosillabiche e in gran parte onomatopeiche; un altro delle voci di origine straniera, introdotte dopo cho le nazioni si furono conosciute tra loro; un terzo, universale, per la scienza del diritto delle genti, dal quale apparisse come gli stessi uomini, fatti o cose, guardati con diversi aspetti dalle varie nazioni, avessero ricevuto diversi vocaboli; e, infine, un dizionario di voci mentali, comuni a tutte le nazioni, che, spiegando le idee uniformi circa le sostanze e le modificazioni diverse che le nazioni ebbero nel pensare intorno alle stesse necessità umane o utilità comuni a tutte, secondo la diversitá dei loro siti, cicli, nature e costumi, narrasse le origini delle diverse lingue vocali, che tutte convengono in una lingua ideale comune.

La seconda fonte, schiusa dal Vico, è l'interpetrazione dei miti o favole, che, conforme alla sua dottrina, non erano allegorie, invenzioni o imposture, ma la scienza stessa dei popoli primitivi. Nel Diritto universale il Vico distinse quattro sensi pei quali gli dèi passarono: dapprima significando cose naturali, Giove il cielo, Diana le acque perenni, Dite o Plutone la terra inferiore, Nettuno il mare, e cosí via; poi, cose umane naturali, per es. Vulcano il fuoco, Cerere il frumento, Saturno i seminati; in terzo 
luogo, fatti sociali; fintanto che, in ultimo, salirono al cielo, furono assunti agli astri, e le cose terrene e umane vennero divise dalle divine. Ma nelle due Scienze nuove mise in rilievo quasi esclusivamente il terzo significato, quello sociale, che diventò per lui l'originario; perché (sembra che egli pensasse) le prime nazioni erano troppo intente a sé stesse, troppo immerse nella loro dura e difficile vita, da speculare astraendo dalle cose sociali. Cosí nei miti egli trovò riflesse le istituzioni, le scoperte, le divisioni sociali, le lotte di classe, i viaggi, le guerre, dei popoli primitivi. Anche pei tempi abbastanza progrediti il Vico $\mathrm{fu}$ alieno dalle interpetrazioni naturalistiche o filosofiche; e il "Conosci te stesso », attribuito all'antico savio, gli parre nient'altro che un monito alla plebe ateniese perclié conoscesse le proprie forze, trasportato dipoi a sensi metafisici e morali. Oltre questa ermeneutica sociale, un altro principio assai importante egli stabilisce: vale a dire che i significati galanti, lubrici e osceni delle favole furono tutti intrusi in tempi tardi e corrotti, che interpetrarono i costumi antichi sui proprî o presero a giustificare le proprie lascivie con l'immaginare che gli dèi ne avessero dato l'esempio. Onde si ebbero Giove adultero, Giunone nemica a morte della virtú degli Ercoli, la casta Diana che sollecita gli abbracciamenti degli addormentati Endimioni, Apollo che infesta fino alla morte le pudiche donzelle, Marte che come se non bastasse commettere adulterî in terra li trasporta fin dentro il mare con Venere, e, peggio ancora, gli amori di Giove con Ganimede e dello stesso Giove trasformato in cigno con Leda: dipinture atte a sciogliere il freno al vizio, come per l'appunto accadde nel giovinetto Cherea dell'Eunuco di Terenzio. Ma nella loro forma e significato originarî le favole furono tutte severe e austere, degne di fondatori di nazioni; e, per es., Apollo che insegue Dafne alludeva agl'indovini o áuspici 
delle nozze, che perseguitavano per le selve le donne ancora in preda ai concubiti vagabondi e nefarî; Venere, che si copre le vergogna col cesto, era simbolo pudico di matrimonî solenni; gli eroi, figliuoli di Giove, non erano già i frutti degli adulterî, ma gli eroi nati da nozze certe e solenni, celebrate con la volontà di Giove che si rivelava negli auspicî. Omnia munda mundis et immunda immundis: le selve e i picchi delle montagne non potevano produrre immagini da alcove e postriboli.

Oltre queste due ricche fonti delle lingue e dei miti, il Vico ne menziona e adopera una terza, che chiama dei « grandi frantumi dell'antichità, cioè delle memorie serbate da storici e da poeti: per es., la tradizione egiziana delle tre età degli dèi, degli eroi e degli uomini; quei quattro o cinque vocaboli che Omero attribuisce alla * lingua degli dèi (ossia, come postilla il Vico, di eroi antichissimi); quegli elenchi di nomi che si trovano in sant'Agostino e il Vico con altri attribuiva a Varrone c che si riferivano ciascuno ad altrettante bisogne della vita naturale, morale, economica e civile dei primi tempi; il luco di Romolo, che Livio definisce « vetus urbes condentium consilium », e alcuni altri pochi aurci detti, « luoghi d'oro », di scrittori antichi. Frantumi finora inutili alla scienza, perché erano giaciuti squallidi, tronchi e slogati, ma che, tersi, composti e allogati, arrecano grandi'lumi. Né (quantunque poco il Vico se ne valga e si veda in fondo che non vi ha troppa pratica) trascura egli di notare i monumenti architettonici o scultorî, dichiarando che come del tempo storico i documenti piú accertati sono le a pubbliche medaglie » ossia le monete, cosí per il periodo favoloso e oscuro sono da tenere in luogo di esse alcuni vestigi restati in marmi », che provano gli antichi costumi: quali le piramidi egiziane descritte di geroglifici e altri frantumi di antichita, che si trovano da per tutto, 
con siffatti caratteri di corpi scolpiti. È degno di nota poi come gli accada perfino di dare esempio di ragionamenti fondati sopra osservazioni di tecnica e condueenti a conclusioni di preistoria; come là dove dice che una prima età del genere umano è contrassegnata dal mangiare carni arrosto, cibo il piú schietto e semplice di tutti perché non ha bisogno d'altro che della brace; e una seconda età, dalle carni lesse, che, oltre del fuoco, hanno bisogno dell'acqua, del caldaio e del tripode.

Metodo potente d'indagine per il Vico è la comparazione, istituita tra gli svolgimenti di cui si ha notizia piú intera e quelli noti imperfettamente o frammentariamente, donde la ricostruzione analogica dei secondi in base ai primi: dimodoché, per es., il principio dell'eroismo, scoperto a evidenza dentro la storia romana, giova a spiegare la storia farolosa dei greci, a supplire la tronca degli egizî e a dare luce alle nascoste di tutte le altre nażioni antiche. Senza negare il fatto delle trasmissioni da un popolo all'altro, il Vico ne satireggiava l'abuso e ne attenuava l'importanza per le società primitive, facendo valere in cambio quello degli svolgimenti spontanei e adoperandosi a ricostruirli col netodo comparativo. Il quale egli intendera in modo assai largo, cioè come da esereitare con materiale raccolto dai piú varî paesi e tempi. Per ispiegare come il cielo fulminante suggerisse ai primi uomini l'idea di un dio, ricorda che gl'indigeni d'America, quando prima udirono lo strepito e videro le stragi delle armi da fuoco in mano agli spagnuoli, credettero clie costoro fossero dèi; i rapsodi dei poemi omerici gli richiamano in mente i cantastorie che sul molo di Napoli recitano i poemi di Orlando e dei paladini; le trasformazioni o metamorfosi cantate dai poeti antichi ravvicina alle fiabe dell'orco e delle fate che ancora le madri narrano per trattenimento ai figliuoletti bambini, o alle favole che nei 
tempi del Medioevo si erano sparse del mago Merlino; la mitologia del focolare è condotta da lui fino alle costumanze del ceppo che in Firenze, ai tempi del Boccaccio, il padre di famiglia accendeva a capodanno sul focolare dando incenso e spargendo vino, e di quello che la plebe napoletana brucia la sera di Natale, nonché all'uso che vigeva altresí nel regno di Napoli di numerare le famiglie per * fuochi »; il serpente Pitone e tutti gli altri serpenti mitici sono messi a riscontro perfino con la biscia « che $i$ milanesi accampa » dei Visconti; i geroglifici coi « rébus de Picardie ", che erano in uso nel settentrione della Francia.

Riuscirebbe vano cercare, nella filologia anteriore 0 contemporanea, cospicui precedenti al complesso di questi canoni, negativi e positivi, che il Vico stabiliva per la storia del tempo oscuro e favoloso; perché essi sono troppo intrinseci a tutto il pensiero filosofico di lui da potere aver avuto altra origine fuori di questo pensiero stesso. I rudi frammenti delle antiche leggi e costumanze e formole romane, i poemi omerici, i vocaboli della lingua latina guardati con occhio sgombro - cioè con quella potenza che ha l'uomo di genio di vedere le cose direttamente, - elaborati da una mente tutta disposta ad appercepirli nella loro vera indole, doverono suscitare nello spirito del Vico, rispetto alla dotta ma incolore o falsamente colorita storiografia del proprio tempo, quella medesima rivolta e rivoluzione, che un secolo dopo fu svegliata nello spirito di Agostino Thierry dalle pagine di poctica prosa dello Chateaubriand, ritraenti i Franchi e Faramondo coi loro gesti incomposti, con le loro rozze e atroci armi, coi loro gridi terribili e con le loro cantilene da barbari. 


\section{XV}

LE SOCIETÀ EROICHE

Appunto come i Franchi apparivano, nei compendî di storia patria pei collegi gesuitici e per le altre scuole di Francia, dispogliati di tutti i loro tratti caratteristici, ridotti a sapienti monarchi, a pie regine e a guerrieri devoti della Chiesa; cosí la storia antica e primitiva, mercé la rettorica e le ingenue idee dei letterati, si era venuta tingendo di luceicanti e falsi colori, press'a poco dei medesimi coi quali allora i Lebrun o i Luca Giordano solevano colorire i loro pomposi e teatrali quadri storici. Re che studiavano saggi provvedimenti per giovare ai popoli e insieme non far mancare alle loro corti lo splendore che irradia una fiorente nobilta, re filosofi, di quelli che avevano fatto sospirare Platone verso $i$ tempi in cui $i$ filosofi regnavano o i re filosofavano; leali e prodi capitani, pronti a sacrificarsi per la comune felicità; uomini di stato che compievano a bella posta viaggi d'istruzione per riportare da lontano le leggi piú ragionevoli ai loro concittadini aspettanti; buơni padri di faniglia, eccellenti madri, giovinetti arditi e obbedienti, fanciulle innamorate e pudiche, personificazione ciascuna di qualche virtú, anzi di tutte le virtú radunate, modelli di umana perfezione; - queste le figure che, rese sacre dalla veneranda antichita, riempi- 
vano i libri e le fantasie. Questi gli eroi della storia greca e romana; e questa fastosa decorazione di cartone clipinto o dorato bisognava stracciare e spazzar via per procedere a ritrovare, nel piú intimo fondo dei ricordi del genere umano, i veri eroi, della realtà e non della letteratura, della vita e non del palcoscenico: gli eroi ignoranti, superstiziosi, feroci, egoisti, duri verso le loro famiglie, spietati verso le plebi, avidi, usurai e, pur tuttavia, anzi per effetto di questi stessi loro aspri atteggiamenti d'animo, eroi, cioè virtuosi di quella virtú che nei tempi primitivi, come era sola possibile, sola era necessaria: la virtú della forza, della disciplina, della cupa e intransigente religiosità.

La falsificazione dell'eroe primitivo nell'uomo saggio e virtuoso dei tempi colti si assommava, per quel che si attiene alla storia politica, nel fraintendimento delle tre parole capitali che compendiano la costituzione dello stato: re, popolo, libertà. Per il fraintendimento della prima si eredeva che la forma originaria dello stato fosse la monarchia assoluta sul tipo del regno di Francia, la monarchia illuminata che si appoggia sulle forze popolari e tiene sottomessi i grandi, la quale è, invece, prodotto tardo, anzi ultimo, della storia. In siffat to errore si era impigliato Giovanni Bodin, verso cui il Vico polemizza di preferenza. Ma il Bodin, piú acuto degli altri trattatisti di politica, si avvolgeva in contradizioni, perché, quantunque entrato nel comune errore, pure, osservando nella sognata liberta romana antica gli effetti di repubblica aristocratica, puntello il suo sistema col distinguere tra stato e governo, e affermare che Roma, ai suoi primi tempi, fu popolare di stato ma si governò aristocraticamente; e poiché neppure questo puntello bastava a sostenere il gran peso dei fatti, finí col confessare che quella repubblica fu aristocratica di governo e di stato, contradicendo cosí a tutta la pro- 
pria cottrina sulla necessaria successione degli stati. Il vero è che ì re di quei primi tempi non erano, a Roma, monarchi, come non furono a Sparta né altrove. Re monarchi, ma di un tipo speciale, soggetti non ad altri che a Dio, imperanti in nome di spaventose religioni col mezzo d'immanissime pene, furono i padri o patrizî o eroi solo al tempo delle monarehie fumiliari, quando eiascuna famiglia viveva da sé. Sorto il primo stato, alleatisi cioè i varî patres familias eroi c costituito per tal modo un ordo, quello patrizio, i loro re non erano altri che uno o piú di essi medesimi, sempliei magistrati dell'ordine. Tantoché Roma, dopo avere in forza di una rivoluzione affatto aristocratiea seaeciato i Tarquinî, non mutò punto di stato; e serbò i re nei due consoli, che erano "reges annui», due re aristoeratici, ai quali « nihil quicquam de regia potestate diminutum ». Lo stesso carattere ebbero i due re di Sparta, sottomessi al pari dei consoli al sindacato e ehe potevano essere dannati a morte dagli efori.

Come falsamente vennero creduti monarchici, falsamente altresí quegli stati furono spaceiati per popolari. Il popolo, del quale a proposito di essi si parla, nou solo non eoineideva con la plebe, ma non la comprendeva in sé: «popolo » era il solo ordine patrizio, e « liberta " la sola libertà dei patrizî, la libertà signorile; la « patria » ben era detta cosi, perelié veramente a res patrum », interesse dei soli padri. É ridicolo pensare ehe la plebe, caterva di vilissimi giornalieri trattati come sehiavi, fosse fornita del diritto di eleggere il re, la cui elezione i padri poi si riserbassero di approvare nel senato. Le relazioni tra padri e plebe consistevano di ben altro che di paeifiea convivenza, scambievole fiducia e coneorde eooperazione. Gli eroi, seeondo una notizia clie il Vico attrịbuisce and Aristotele, prestavano solenne giuramento di essere eterni nemici della plebe: ccco il loro spirito democratieo! E la 
* romana virtí », che offre tanti e tanti esempî gloriosi, nessuno ne offre di pietà verso la plebe. Bruto, che consacra con due suoi figliuoli la sua casa alla liberta; Scevola, che col punire del fuoco la sua destra, atterrisce Porsenna; Manlio l'imperioso, che per un felice peccato di disciplina militare fa mozzare la testa al suo figliuolo reduce da una vittoria; i Curzî, che si gettano armati a cavallo nella fossa fatale; i Decî, che si consacrano per la salvezza dei loro eserciti; i Fabrizî e i Curî, che rifiutano le some d'oro dei sanniti e i regni di Pirro; gli Attilî Regoli, che vanno a certa morte per serbare la santità romana dei giuramenti; - che cosa fecero a pro della plebe, se non sempre piú angariarla nclle guerre, sempre piú profondamente sommergerla nel mare delle usure, sempre piú seppellirla nelle prigioni private dei nobili, dove i plebei erano battuti a spalle nude a guisa di vilissimi schiavi? E guai a colui tra gli aristocratici che avesse lasciato scorgere la piu piccola voglia di alleviare quei mali! Era subito accusato ribelle e traditore e messo a morte; come accadde in Roma a Manlio capitolino, che aveva salvato il Campidoglio dall'incendio dei Galli e che pur tuttavia, per le sue simpatie democratiche, fu fatto precipitare dal monte Tarpeo; e accadde in Isparta (la citta degli eroi di Grecia, come Roma fu degli eroi del mondo) al magnanimo re Agide, il Manlio capitolino di cola, il quale, perché aveva tentato di sgravare la povera plebe lacedemone con una legge di conto nuovo e di sollevarla con un'altra testamentaria, fu dagli efori fatto strozzare. La celebrata « virtú romana * sbalordisce chi vi si appressa con le idee moderne di una virtú consistente nella giustizia e nella benevolenza verso il genere umano. Quale virtú dove fu tanta superbia? quale moderazione dove tanta avarizia? quale mansuetudine dove tanta fierezza? quale giustizia dove tanta inegualità? 
Allo stesso modo che verso la plebe, durissimi procedevano gli eroi verso le proprie famiglie. L'educazione dei fanciulli era severa, aspra, crudele: gli spartani, al fine di avvezzarli a non temere dolori e morte, battevano nel tempio di Diana i loro figliuoli fino all'anima, talché sovente, convulsi dal dolore, cadevano morti sotto le bacchette dei padri. In Grecia come in Roma era permesso di uccidere gl'innocenti bambini di fresco nati, diversamente dai tempi moderni nei quali le delizie di che si circondano i figliuoli fanciulli formano la delicatezza della natura umana. Le mogli erano comperate con le doti eroiche (donde il costume, che rimase per solennita in Roma, dei matrimonî "coëmptione et farre", di cui il simile viene narrato da Tacito degli antichi Germani e si deve stimare che fosse di tutti i popoli barbari); ed erano tenute per sola necessita di natura, a uso di fare figliuoli, e, del resto, trattate come schiave: il che si vede ancora in molte parti del vecehio e quasi dappertutto nel nuovo mondo. Gli acquisti dei figliuoli, i risparmî delle mogli andavano a esclusivo profitto dei loro padri e mariti.

Rispondevano a siffatta costituzione politica e familiare gli abiti di vita, ignari di lussi, lautezze ed agi. I giuochi erano faticosi, come lotta e corsa, per fermare le forze e gli animi; o pericolosi, come giostre e cacce di fiere, per adusare al disprezzo delle ferite e della morte. Le guerre si conducevano cone guerre di religione, e percio tutte atrocissime. Conseguenza di esse le schiavitú eroiche, nelle quali i vinti erano tenuti uomini senza Dio, onde con la libertà eivile perdevano anche quella naturale. Gli stranieri erano considerati nemici: le prime nazioni furono sommamente inospitali. Brigantaggio e corseggio, ammessi; e Plutarco (o, veramente, Giustino) dice che gli eroi si recavano a grande onore e si riputavano in pregio d'armi con l'esser chiamati * ladroni ». 
Era, insomma, una società uscente immediatamente da quell'età degli dèi che, come sappiamo, fu la crisi dello stato ferino. Usciva dalla preistoria, come si direbbe modernamente, ed entrava per la prima volta nella storia, ritenendo assai degli anteriori costumi, di quelli che il Vico (pensando ai solitarî Polifemi delle grotte) chiamava « imperi paterni ciclopici ». Usciva dall'eta dell'oro, dall'età dell'innocenza, tanto mansueta, benigna, discreta, comportevole e doverosa, come vogliono i dotti e i poeti, e che in realta fu tutto un « fanatismo di superstizione ", agitata dal continuo terrore della divinità, alla quale per placamento offriva vittime umane (di che si hanno tracce presso i fenici, gli sciti, i germani, i popoli delle Americhe, e presso i romani stessi, che li sostituirono piú tardi con la cerimonia del gittare fantocci di giunco nel Tevere): sacrificavano perfino i proplî figliuoli, come ne serba ricordo, tra l'altro, il sacrificio che Agamennone offerse d'Ifigenia. Ma in quella età degli dèi, attraverso e per opera di questa crudele superstizione, si fondarono le grandi istituzioni umane: il culto religioso con la divinazione per auspicî, i matrimonî, le sepolture. Nozze, tribunali ed are, e il togliere all'etere maligno e alle fiere i corpi degli estinti, "diero alle umane belve esser pietose », come disse poi il Foscolo nei Sepolcri, verseggiando per l'appunto la prosa del Vico. Quei « ciclopi », che adunavano e confondevano in sé gli ufficî del re; del sapiente (sapiente in divinazione) e del sacerdote, avevano posto dapprima le loro sedi sulle alture dei monti, in luoghi d'aria ventilata e perciò sana, in siti naturalmente forti, presso alle fontane perenni, dove erano nidi di aquile e di avoltoi (gli uccelli, dai quali furono tratti gli auspicî). Donde l'importanza dell'acqua e del fuoco, diventati simboli della famiglia; e "aqua et igni si strinsero i primi matrimont, tra coloro che avevano l'acqua e il fuoco comuni, cioè ap- 
partenevano alla stessa famiglia, sicché dovettero cominciare tra fratelli e sorelle. Eta fortemente morale, codesta dei ciclopi; sebbene non quale la finsero dipoi i poeti effeminati che licesse ciò che piacesse, perché a quegli uomini, storditi ad ogni gusto di nauscante riflessione » (come si può anche oggi vedere da chi osservi il costume dei contadini), non piaceva se non ciò che cra lecito, e non era lecito se non cio che giovava. Erano giusti, della selvatica giustizia verso il loro dio; temperanti, perché avevano smesso il concubito ferino; forti, industriosi e magnanimi, come di necessità in quelle difficili e rischiose condizioni di vita. Solo piú tardi quei primi aggruppamenti umani si cstesero alle pianure, che cominciarono a coltivare; e da mediterranei che erano in origine, via via scesero alle marine, e navigarono e dedussero colonie.

Le famiglie, le «genti » erano dunque prima delle « città », e le città furono costituite dalle genti, collegate in ordine, dalle «gentes maiores " o « case nobili antiche», come vennero poi chiamate per distinguerle dalle altre accolte in séguito nell'ordine (per es., ai tempi di Giunio Bruto, per riempire i vuoti del senato romano dopo la cacciata dei re), le quali si chiamarono invece "gentes minores ». Ma quelle genti o famiglie avevano in sé un elemento di differenziazione e di lotta. Le famiglie non erano composte (come, pel solito errore di dare a vocaboli antichi significatì moderni, si è generalmente creduto) dalle sole mogli e figlinoli, ma altresi dai famoli, da coloro che, meno forti e perduranti piú a lungo nel divagamento ferino, finalmente, come le fiere talvolta, o pel gran freddo o inseguite dai cacciatori, per campar la vita si riparano in luoghi abitati, avevano chiesto rifugio presso i piú forti, nelle ròcche dei padri. Essi, in cambio della ricevuta protezione, lavoravano le terre dei padri, alle quali furono come affissi e annodati, onde si appellarono anche 
"nexi »; li seguirono e serrirono, e si dissero perciò "clientes". La società dei famoli coi padri fu la seconda forma di umano consorzio, dopo quella naturale del matrimonio; e costitui il consorzio feudale, che a torto si è creduto particolare di alcuni tempi barbari, cioè del Medioero, giacché esistette in tutte le società eroiche e fu l'eterno principio dei feudi onde nacquero tutte le repubbliche al mondo. Come Tacito dice parlando dei Germani, a questi famoli e clienti a suum principem defendere et tueri, sua quoque fortia facta gloria eius adsignare, procipuum iuramentum erat ": che è una delle proprieta piú spiccanti dei feudi. Del resto, non tanto i figli degli eroi si distinguevano dai famoli quanto piuttosto vi si confondevano: si distinguevano nel nome, "liberi ", ma si confondevano nella eguale comune obbedienza e nella mancanza di propria personalità.

La necessita, in cui si trovarono i padri di garantirsi contro i famoli che sovente si ammutinavano, dié origine all'alleanza dei padri tra loro, all'ordine patrizio e alla citta eroica. Della quale citta i famoli formarono la prima plebe, senza diritti di cittadini, perché tali essi non erano, né di matrimonî solenni, perché gli auspicî stavano solo presso i padri, né di testamenti, perché i testamenti avevano e serbarono carattere politico d'imperio. Ed erano perciò esclusi dai comizî curiati, che si tenevano dai padri sotto le armi e che restarono dipoi per trattare le cose sacre; sotto il quale aspetto si guardavano in quei primi tempi dappertutto (in Roma, come nell' Egitto o nella Germania) anche le cose profane. I re dei patrizî, che abbiamo detto magistrati dell'ordine, furono quindi, piú particolarmente, i loro conduttori e capitani nella resistenza da opporre ai famoli o plebei.

Ma gli eroi non provvidero alla stabilità del loro ordine con la solà resistenza, fatta di forza; e come essi, 
sovrani nelle loro famiglie, quando si assoggettarono alla maggiore sovranita dell'ordine formarono una specie di feudi nobili o armati, cosi per tenere contenti in qualche modo i famoli all'obbedienza, concessero loro, pur senza accoglierli nella cittadinanza, una specie di feudo rustico. L'origine della proprieta appare in tal modo tutta diversa da quella leggiadramente poetica, secondo la quale gli uomini, ornati dalle virtú del secolo d'oro quando la giustizia dimorava sulla terra, prevedendo i disordini che sarebbero potuti nascere dalla comunione, essi stessi sarebbero stati benigni arbitri nel tracciare i confini dei campi, cercanclo che ad altri non toccassero terre tutte fertili, ad altri tutte infeconde, ad altri affatto assetate, ad altri abbondanti di acque perenni!; e diversa altresi dall'origine "filosofica » di una volontaria sottomissione ai sapienti, e infine da quella, escogitata da * rei politici », che fanno nascere la proprietà dalla violenza. - La concessione dei feudi rustici, che può dirsi la prima legge agraria, distinse i tre dominî, il bonitario o dei soli frutti concesso dai patrizî ai plebei, il quiritario o nobile conservato con le armi presso i padri, e l'eminente, che spettava all'ordine tutto, cioè a tutto lo Stato patrizio. E perché nella ricchezza era riposta la forza dell'ordine, le repubbliche eroiche, per serbare la ricchezza presso i nobili, si guardavano a tutto potere dall'arricchire le plebi, e nelle guerre (ecco la ragione politica della «clemenza romana ) toglievano ai vinti le sole armi e, imponendo un tributo comportevole, rilasciavano loro il dominio bonitario delle terre. Per la medesima ragione, i patrizî erano rattenutissimi di venire alle guerre, nelle quali la moltitudine dei plebei si agguerriva e si faceva pericolosa.

Anche le leggi solo lentamente si staccarono dalla forza, pur ritenendone in ogni lor parte l'impronta e il ricordo. Nelle repubbliche eroiche (afferma il Vico, anzi fa affermare 
da Aristotele) non r'erano dapprima leggi da punire le offese e ammendare i torti privati; onde, mancando le leggi giudiziarie, ne veniva la necessità dei duelli e delle rappresaglie, che perpetuavano le costumanze dell'età dell'innocenza o età degli dèi. La poesia e la storia ci descrivono alcuni di questi duelli, che erano giudizî armati, come quello di Menelao e Paride sotto le mura di Troia e quello degli Orazî e Curiazî tra Roma ed Alba. Consiglio della provvidenza divina, acciocchẻ tra genti barbare e di cortissimo raziocinio, che non intendevano ragione, da guerre non si seminassero guerre, e il torto e il diritto fossero in qualche modo discriminati dal credere di avere propizî o avversi gli dèi, secondo la vittoria o la sconfitta.

Ai giudizî armati si accompagnarono e seguirono giudizî per mezzo di formole verbali, le quali per l'abito religioso degli animi si adoperavano con iscrupolo sommo di osservanza, badando a non alterarle pur d'una lettera (religio verborum). Orazio, che, per l'uccisione della sorella incorse nella legge «horrendi carminis », non poté essere assoluto dai decemviri, benché lo tenessero innocente; e il popolo l'assolse (dice Livio) « magis admiratione virtutis quam iure causa ». Anche piú tardi il diritto romano ritenne tanto di questi scrupoli di parole che esso appunto porse argomento a piú commedie di Plauto, nelle quali i lenoni sono raggirati dai giovani innamorati, che li fanno trovare rei in qualche formola di legge.

Il diritto privato rispondeva pienamente alla costituzione economica di quelle societa, che era del tutto naturale, ristretta alle cose necessarie della vita, senza uso di danaro; e però il diritto non conosceva i contratti che poi si dissero compiersi col solo consenso. Tutte le obbligazioni si assicuravano con la mano, le prime compere furono permutazioni, le locazioni di case consistevano in 
censi di suoli da fabbricare, le locazioni dei terreni in enfiteusi, le società e i mandati erano ignoti.

La materialità dei primi contratti e la violenza dei primi processi furono via via temperate, e diventarono simboliche. Come le finzioni della forza nei riti matrimoniali rammentava la forza vera onde i giganti strascinavano le prime femmine dentro le grotte; cosi, egualmente, le cerimonie della mancipazione, dell'usucapione, della rivendicazione erano state dapprima atti realmente eseguiti. La mancipazione si compieva, come si è detto, con vera mano ossia con forza effettiva (per es., nella occupazione, prima fonte di tutti i dominî); l'usucapione si celebrava col continuo ingombramento dei corpi sopra esse cose possedute; le rivendicazioni furono duelli, e le «condictiones », rappresaglie private. Divennero poi cerimonie o favole: la mancipazione, tradizione civile con atti e parole solenni (si quis nexum faciet mancipiumque, uti lingna nuncupassit ita ius esto); l'usucapione, possesso che si finge ritenersi con l'animo; le rappresaglie, azioni personali con la solennita di denunziarle o intimarle al debitore. Si portarono in piazza tante maschere quante erano le persone, e sotto la persona o maschera di un padre di famiglia si nascondevano tutti i figliuoli e tutti i servi della casa. Invece delle forme astratte, che ancora mancavano, si ebbero forme corporee animate: l'Ereditá, per es., fu finta domina delle robe ereditarie e in ogni particolare cosa creditata fu ravvisata tutta intera; l'idea del diritto indivisibile si materializzava nella gleba o zolla di terra, che si presentava al giudice con la formola "hunc fundum *. Quell'antica giurisprudenza era tutta poetica: fingeva i fatti non fatti, i non fatti fatti, nati i non nati ancora, morti i viventi, $i$ morti vivi nelle loro eredita giacenti. Introdusse tante vane maschere senza soggetto (iura imaginaria), ragioni favoleggiate da fantasia; e le formole, con le quali parlavano 
le leggi, per le loro circoscritte misure di tali e tante parole si dissero "carmina». I frammenti delle dodici tavole, se bene vi si rifletti, nella piú parte dei loro capi vanno a terminare in versi adonî, che sono ultimi ritagli di versi eroici; e Cicerone per imitazione iniziò un paragrafo del De legibus col verso: «Ad Divos adeunto caste, pietatem adhibento ». Nello stesso Cicerone il Vico riusciva a trovare che $i$ fanciulli romani andavano cantando le leggi delle dodici tavole «tamquam necessarium carmen »; il medesimo trovara in Eliano a proposito dei fanciulli cretesi e delle leggi di Minosse; e in altri autori, che le leggi egiziane, secondo una tradizione, erano a poemi della dea Iside , e che in versi o in prosa ritmiche furono formolate quelle che Licurgo diede agli spartani e Dracone agli ateniesi. Tutto il diritto romano antico fu un a serioso poema », o (come il Vico dice altrove) « poëma quoddam dramaticum romanorum », clic si rappresentava dai romani nel fòro; e l'antica giurisprudenza, una severa poesia.

Questa atmosfera poetica delle società eroiche, e questo atteggiarsi metrico dei loro linguaggi, trovano conferma in molte testimonianze e documenti, in osservazioni e congetture di dotti, in ragguagli di viaggiatori e missionarî. Gli ebraisti sono divisi d'opinione intorno all'essere composta la poesia ebraica di metri o di ritmi; ma Flavio Giuseppe, Origene, Eusebio (diceva il Vico, facendo sua un'affermazione di san Girolano) stanno a favore dei metri, e lo stesso san Girolamo vuole tessuta in verso eroico gran parte del Libro di Giobbe. Gli arabi, ignoranti di lettere, conservarono la propria lingua con tenere a memoria $\mathbf{i}$ poemi nazionali, tino al tempo in cui inondarono le provincie orientali dell'impero greco. In versi scrissero gli egizî le memorie dei defunti; in versi consegnarono le prime Ioro storie $\mathrm{i}$ persiani e $\mathrm{i}$ cinesi in versi altresí (secondo Tacito) i germani e (secondo afferma Giusto Lipsio) 
gli americani; e poiché, delle due ultime nazioni, la prima non fu conosciuta se non assai tardi dai romani e la seconda dagli europei solo alla fine del secolo decimoquinto, si ha forte argomento di congetturare il medesimo per tutte le altre nazioni barbare, antiche e moderne.

Il primo verso, che si ritrova non solo presso i greci ma presso gli assiri, i feniei e gli egizî, fu quello eroieo; e per la tardezza delle menti e per la difficoltà della pronunzia dové nascere dapprima spondaico (onde non lasciò poi mai nell'ultima sede lo spondeo), e solo di poi, facendosi piú spedite le menti e le lingue, ammise il dattilo. In séguito ancora, spedendosi lingue e menti vieppiú, nacque il verso giambico (di piede presto, come dice Orazio), il piú somigliante di tutti alla prosa; tanto che i primi prosatori, anteriori a Gorgia, usarono numeri quasi poetici ossia giambi, e di frequente la prosa scivola in questi. In giambo fu verseggiata la tragedia, alla quale quel verso convenne per natura, perché nato per isfogare la collera (come di Archiloco si narra che l'avesse ritrovato per isfogure la sua contro Licambe); e se del giambo si valse poi anche la commedia, fu per una «vana osservazione d'esemplo e non perché esso le fosse connaturato come alla tragedia.

Oltre che per il metro, i primi linguaggi di quelle società erano poetici perché tutto il loro corpo si componeva di metafore attuose, d'inmagini vive, di somiglianze evidenti, di comparazioni acconce, di espressioni per gli effetti o per le eagioni, per le parti o per gl'interi, di ellissi o.parlari difettosi, di pleonasmi o parlari soverehi, di onomatopeie o imitazioni di voei a suono, di accorciamenti, di parole congiunte, di circonlocuzioni minute, di aggiunti individuati, di contorsioni sintattiche e di episodî. Tutti modi di farsi intendere, trovati da coloro ehe ignoravano le voci proprie o ehe nel parlare con altrui mancavano di 
voci intese da ambe le parti. Gli episodî infatti sono proprî degli ignoranti e delle donnicciuole, che non sanno trascegliere delle cose ciò che a essi bisogna né tralasciare ciò che non appartiene al loro proposito; il parlare contorto è naturale effetto di chi non sappia o sia impedito di spiegarsi tutto, come si può osservare negli irati e dispettosi che proferiscono i casi retto e obliquo e tacciono i verbi. Persino le singole parole di quei linguaggi recano, nella loro abbondanza di dittonghi, traccia del cantare onde sorse il parlare; e cosi ricche di dittonghi rimasero nelle lingue greca e francese, le quali passarono rapidamente e immaturamente dall'eta spontanca alla riflessa. Un tesoro di forme eroiche deve offrire la lingua tedesca, con la sua ricchezza di voci composte che cosi felicemente traducono quelle greche e con la sua sintassi tanto piú raggirata della latina quanto la latina è piú della greca. Se i dotti di essa lingua (ripete piú volte il Vico, guardando con bramosia il territorio della germanistica a lui inaccessibile) attenderanno a trovarne le origini mercé i principî della Scienza nuova, vi faranno certamente discoperte maravigliose.

Poetica o, per dir meglio, mitica fu altresí, presso quelle socictà, la concezione del mondo, e poetiche le storie che narrarono di sé medesime, e cioè delle loro origini e delle loro lotte e vicende; anzi, come si è visto, le loro poctiche concezioni di storia sociale precedono le loro concezioni cosmologiche, fisiche e psicologiche. Applicando inflessibilmente questo criterio, il Vico svolse la sua teogonia naturale, sorta naturalmente nelle fantasie a certe occasioni di umane necessità e utilità: la genesi dei dodici dèi maggiori, ossia di quelli che le genti maggiori finsero e in gran parte portarono seco allorché fondarono le città. Giove o il Cielo fu, col suo parlare per cenni di fulmini, il datore delle prime leggi familiari; Giunone sim- 
boleggio le nozze; Diana, la castità dei connubî; Apollo, la luce civile; Vulcano, Saturno e Cibele, rispettivamente il fuoco appiccato alle selve per diradarle, la seminagione e la cultura delle terre; Marte, il combattere eroico \& pro aris et focis »; Venere, la bellezza civile. Alla quale Venere « celeste » sorse accanto una Venere plebea, cui si attribuirono le colombe, non gia per significare svisceratezze amorose, ma perché «degeneres », uccelli vili a petto delle aquile; e un duplice significato, patrizio e plebeo, fu dato parimente a Vulcano e a Marte. Nelle dodici deita maggiori cominciarono a riflettersi le tempestose relazioni dei padri coi famoli, alle cui lotte e sofferenze alludono i miti di Tantalo, d'Issione e di Sisifo. Ercole (le cui dodici fatiche sono storie poetiche di dodici diverse età) in quanto lotta con Anteo è carattere dei nobili delle città eroiche, come Anteo dei famoli ammutinati che vengono ricondotti nelle prime citta poste sulle alture (Anteo sollevato in aria), e vinti e annodati alla terra, e cioè costretti al lavoro servile. La nascita della decima divinità, Minerva, esprime l'infiacchimento o diminuzione del potere eroico, perché Vulcano plebeo (i famoli ammutinati) con una scure (strumento di chi esercita arti servili) fende il capo a Giove. Mercurio rappresenta invece la comunicazione del dominio bonitario ai plebei e il mantenimento del quiritario ai padri. L'ultima delle dodici divinita, Nettuno, sorge nel tempo della discesa delle genti alle marine, e le favole di Minosse, degli argonauti, della guerra troiana, del ritorno di Ulisse, di Europa e del toro, del Minotauro, di Perseo, di Teseo, ricordano le colonie e i corseggi.

Il mitologizzamento della storia prosegue anche dopo la fondazione della città. $E$, se non dèi propriamente, caratteri poetici sono quelli dei fondatori della civilta, di Zoroastro o dei due Mercuri Trismegisti, di Orfeo o di Confucio. Esopo è carattere dei socî o famoli degli eroi, e 
perciò fu detto brutto ossia privo di bellezza civile (honestas); e dalla favola esopica della societa leonina traspare a maraviglia la realtà del consorzio degli eroi coi famoli, messi a parte delle fatiche ma non poi delle prede. Dracone, del quale non altro si sa dalle storie greche se non che impose leggi severissime, simboleggia la crudelta degli eroi verso i famoli. Solone o fu un capoparte della plebe di Atene o, addirittura, un'idea degli stessi plebei ateniesi, considerati sotto l'aspetto delle loro rivendicazioni. Pitagora e i pitagorici, nonché di sublimi filosofi, formarono setta politica di « nobili », i quali, avendo attentato di ridurre le Ioro repubbliehe da popolari in aristocratiche, furono tutti tagliati a pezzi. Caratteri poetici sono, nella storia romana, Romolo, al quale vennero attribuite tutte le leggi intorno agli ordini; Numa, autore di quelle intorno alle cose sacre e alle divine cerimonie; Tullo Ostilio, delle leggi e ordinamenti di disciplina militare; Servio Tullio, del censo (che, contrariamente alla veriti storica, venne supposto fondamento di repubblica popolare, laddove fu di aristocratica); Tarquinio Prisco, delle insegne e divise; perfino il decemvirato e le dodici tavole (intorno alle quali il Vico scrisse una speciale dissertazione) diventarono caratteri poetici, perché a questi avvenimenti e personaggi furono riportate moltissime delle leggi posteriori agguagliatrici della libertà.

Cosí, prima che i filosofi prendessero a elaborare le favole e, sotto l'illusione d'interpetrarle, effettivamente ne creassero di nuove, - e Platone, per es., introducesse in Giove l'idea del suo etere che scorre e penetra tutto, e altri in Minerva che nasce dal capo di Giove vedessero raffigurata l'idea eterna di Dio generata in esso Dio, a contrasto delle idee negli uomini che sono prodotte da Dio, o nel Caos e nell'Orco la confusione dei semi universali della natura e la prima materia delle cose, - i poeti teologi vi 
avevano espresso le loro idec, poco o niente metafisiche e fisiche, nudrite tutte di casi umani e politici. Il Caos dei poeti teologi era la confusione dei semi umani nello stato dell'infame comunione delle donne: confuso perché senz'ordine di umanita, oscuro perché senza luce civile. L'Orco, mostro informe, divorava ogni cosa, perché gli uomini in quella comunione non avevano forme d'nomini ed erano assorbiti dal nulla, non lasciando, per l'incertczza della prole, nulla di sé. I quattro elementi del mondo rispondevano ai quattro elementi della vita sociale: l'aria dove fulminava Giove, l'acqua delle fonti perenni, il fuoco onde le selve furono bruciate, e la terra dove si esercita il lavoro umano. L'essere e il sussistere erano concepiti il primo come il « mangiare » (ancora i contadini, per dire ehe l'ammalato è in vita, dicono che « mangia $»$ ), e il secondo come lo « stare sui talloni ». La compagine del corpo era ridotta ai solidi e ai liquidi, l'anima all'aria, la generazione al "concipere 0 "concapere ", ossia prendere d'ogni intorno i corpi vicini, vincerne la resistenza e adeguarli e conformarli alla propria natura; e tutte le operazioni interne dell'anima, al capo, al petto e al cuore.

Anguste, perché ristrette alla vita di quelle società, erano le idee cosmografiche. Il primo ciclo non fu messo piú in su delle alture rei monti, dove i giganti videro scoppiare i fulmini; l'inferno non fu piú profondo di un fosso, e soltanto a poco a poco venne ampliato e profondato nelle valli, come opposte al cielo, ossia alle cime dei monti; la terra s'identificava coi confini dei campi coltivati. Col progresso dei tempi il ciclo, oggetto di contemplazione per trarne augurî, si alzò piú in su, e con esso piủ in su gli dèi c gli croi, i quali furono affissi ai pianeti e alle costellazioni, c si ebbe cosí l'astronomia poctica. Poetica del pari era la cronologia primitiva, nella quale gli anni erano noverati con le raccolte del frumento, come 
mostrano il Post aliquot, mea regna, videns mirabor aristas e il tertia messis erat virgiliani, e anche l'uso odierno dei contadini toscani, che, invece di «tre anni », dicono « abbiamo tre volte mietuto ». La conoscenza geografica non si estendeva di la dal paese abitato da ciascuna nazione: che è la ragione per la quale i popoli, portandosi in terre straniere e lontane, dettero alle nuove citta e ai nuovi monti, colli, stretti, isole e promontorî i medesimi nomi, che avevano quelli della loro patria d'origine. L'Asia o l'India fu dapprima, pei greci, la parte orientale della Grecia stessa; l'Europa o l'Esperia, quella occidentale; la Tracia o la Scizia, la settentrionale; la Mauritania (donde il Vico faceva derivare il nome di Morea), la meridionale.

Ma noi non esporremo altri particolari (e moltissimi ne abbiamo già tralasciati), perché non è nelle particolari determinazioni il pregio del quadro che il Vico dipinge dell'etả eroica. Etimologie, interpetrazioni mitiche, genesi e successione cronologica delle deită, genesi e successione delle forme fonetiche, metriche o stilistiche, tutte sono contestabili una per una, tutte o quasi poggiano su fondamenti eruditi alquanto fragili, ma, dal loro complesso, si sprigiona una verità che va oltre le particolari affermazioni. Il loro complesso è il possente sforzo di rievocare una forma di società e di umanità vivente senza dubbio nei ricordi.e nei monumenti sopravanzati, riconoscibile ancora qua e là frammentariamente in alcune parti del mondo moderno, ma che per secoli, e ancora ai tempi del Vico, era stata come sepolta sotto un cumulo d'immagini estranee, di paradigmi convenzionali, di pregiudizî d'ogni sorta, che impedivano di scorgerne i lineamenti genuini. 


\section{XVI}

OMero e la POESIA PRIMItTIVA

Della società eroica fu poeta Omero; e se tale egli fu, non gli poté appartenere quella profonda saggezza, quella squisita ed elevata moralita, quella somma perizia in tutte le scienze e arti piú sublimi, che i filosofi e gli scrittori antichi avevano immaginato di lui, e che l'opinione comune dei letterati e critici del secolo decimosettimo ancora gli attribuiva.

Quale stravagante filosofo sarebbe stato Omero, se fosse stato filosofo; quale stolto ordinatore della greca civilta, se avesse inteso a ordinare civiltà! II suo Giove adduce la forza, la forza brutale, per misura della riverenza che bisognava avergli; la sua Minerva, non contenta d'avere stesa a terra Venere, percuote Marte con un sasso, Marte a sua volta la chiama mosca sfacciata, Giunone strappa il turcasso a Diana e glielo rompe sulla schiena; un semplice mortale, Diomede, ferisce Venere e Marte. Gli eroi Achille e Agamennone si scagliano l'un l'altro contumelie, quali ora appena userebbero i servi nelle commedie, dicendosi « occhi di cane » e « cuore di cervo »; e contendono nel modo piú rozzo per le Briseidi e per le Criseidi. Fieri di costumi, abbandonano i cadaveri dei nemici al pasto dei cani e corvi; intemperanti nel godere, si ubbria- 
cano. Altezza d'intelletto, gentilezza d'animo, equilibrio spirituale si cercherebbero indarno in tutti i loro atti e sentimenti. Quegli eroi si dimostrano, invece, di cortissimo intendimento, di vastissima fantasia, di violentissime passioni, zotici, crudi, aspri, fieri, orgogliosi, diffidenti e ostinati nei propositi e, al tempo stesso, mobilissimi a ogni nuovo oggetto che loro si presenti innanzi. Anche qui il riscontro piú calzante può essere offerto dalla psicologia dei contadini; i quali, come giornalmente si osserva, a ogni motivo di ragione che loro si dica, si rimettono, ma, perché deboli di raziocinio, lasciando subito sfuggire il concetto che li aveva persuasi, tornano facilmente al primo proposito. Parimente gli eroi omerici talora, al primo detto che si odono opporre, si acquetano; tal'altra, nel bollore della collera, rimembrando a un tratto cosa lagrimevole, si dileguano in amarissimi pianti; o, mentre sono afflitti da somma angoscia, presentandosi loro innanzi cosa lieta (come a Ulisse la cena di Alcinoo) si dimenticano affatto di guai e tutti si sciolgono in allegria; 0 , infine, mentre stanno riposati e quieti, a un'innocente parola altrui che loro non vada all'umore montano in si cieca collera da minacciare presente atroce morte a chi la profferi. Anche le virtú che sono loro proprie in sommo grado, quel loro animo aperto, risentito, magnanimo e generoso, reca impresso il medesimo carattere di passionalità e d'irriflessione.

L'croe degli eroi, Achille, colui che porta seco i fati di Troia, per una privata offesa fattagli da Agamennone (la quale, per grave che fosse, non era giusto vendicare con la rovina della patria e di tutta la nazione), si compiace che vadano in perdizione tutti i greci, battuti miseramente da Ettore. Né si risolve a recar loro soccorso se non per dare soddisfacimento a un suo privato dolore, di averc Ettore ucciso il suo Patroclo. E almeno foss'cgli venuto in quell'estremo sdegno per passione d'amore e per 
gelosia! Ma per la Briseide toltagli da Agamennone, - e per la quale fa tanto fiacasso da empirne la terra e il cielo e porgere la materia perpetua all'Iliade, - Achille non mostra, in tutto il corso del poema, pur un menomo senso di affetti amorosi; come di questi non dà il piú piccolo segno Menelao, che per Elena rapitagli muove Grecia tutta contro Troia, ma non mai si tormenta di gelosia perché Paride se la goda. Privo di ogni senso di umanita, Achille a Ettore che vuole patteggiare con lui la sepoltura se nell'abbattimento l'uccida, nulla riflettendo all'egualità del grado, nulla alla morte che tutti agguaglia, feroce risponde: «Quando mai gli nomini patteggiarono coi leoni, o i lupi e gli agnelli ebbero uniformita di voleri?»; e * anzi » (soggiunge), "se t'avlò ucciso, ti trascinerò nudo legato al mio cocchio per tre giorni d'intorno alle mura di Troia " (come realmente poi fece); e, finalmente: " ti darò a mangiare ai miei cani di caccia ». E questo avrebbe pur fatto, se l'infelice padre Priamo non fosse venuto da lui a riscattarne il cadavere. Ma, anche in quell' incontro sommamente pietoso (continua il Vico, rifacendo alquanto a suo modo il testo omerico), ricevuto Priamo nella sua tenda, il quale di notte, con la scorta di Mercurio, era passato tutto solo per mezzo al campo dei greci, e ammessolo a cenar seco, per un sol detto che all' infelice vecchio cade involontariamente di bocca lamentando la perdita di sí valoroso figliuolo, dimentico delle santissime leggi dell'ospitalità, non rattenuto dalla fede onde Priamo si era tutto a lui affidato, nulla commosso dalle molte e gravi miserie di un tal re, nulla dalla pieta di un tal padie, nulla dalla venerazione di un tanto vechio, nulla riflettendo alla fortuna comune della quale non vi ha cosa che piú vaglia a muovere compatimento, montato in una collera bestiale gl' intuona sopra clue "gli vuol mozzare la testa »! Della Briseide toltagli nemmeno morto si placa, se l'infelice bellis- 
sima real donzella Polissena, della rovinata casa del poc'anzi ricco e potente Priamo, divenuta misera schiava, non gli venga sacrificata sul sepolero e le suc ceneri assetate di vendetta non bevano l'ultima goccia di quel sangue innocente. E giú nell'inferno Achille, domandato da Ulisse come vi stia volentieri, risponde che « vorrebbe essere un vilissimo schiavo, ma vivo $» !$ Questo è l'eroe che Omero,

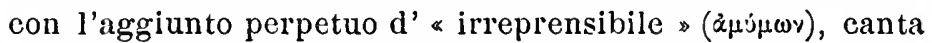
ai popoli greci in esempio della virtú eroica. Un siffatto eroe, che pone tutta la ragione nella punta della lancia, non si può altrimenti intendere se non come un uomo orgoglioso, il quale ora si direbbe che non si faceia passare la mosca per innanzi alla punta del naso.

Se i piú grandi caratteri di Omero sono tanto sconvenevoli alla nostra natura civile, le comparazioni delle quali egli si vale hanno a lor materia belve e altre cose selvagge. E se per i costumi che rappresenta - da fanciulli per la leggerezza delle menti, da femmine per la robustezza della fantasia, da violentissimi giovani per il fervido bollore della collera, - e per le favole degne di vecchierella che intrattenga bimbi ond'è piena l'Odissea, non si può attribuire a Omero nessuna sapienza riposta; quel suo cotanto riuscire nelle fiere comparazioni non $\grave{e}$ certamente da ingegno addimesticato e incivilito da alcuna filosofia. Né da animo che sia umanato e impietosito da filosofia potrebbe nascere quella truculenza e fierezza di stile, onde si descrivono tante e sí varie e sanguinose battaglie, tante e sí diverse e tutte in istravaganti guise crudelissime specie di ammazzamenti, che particolarmente formano la sublimità dell'Iliade.

Ma ehi fu, in realtà, Omero? Che cosa di lui dicono gli antichi scrittori, che cosa si trae dai suoi poemi? A leggere l'Iliade e l'Odissea senza pregiudizî, a ogni passo ci si avventano agli occhi e ci offendono stravaganze $\mathrm{e}$ in- 
coerenze. Incoerenze di costumi, che trasportano or di qua or di la a tempi lontanissimi tra loro: da una parte si vede Achille, l'eroe della forza; dall'altra, Ulisse, l'eroe della saggezza; da una parte, la crudezza, la villania, la ferocia, l'atrocità; dall'altra, i lussi di Alcinoo, le delizie di Calipso, i piaceri di Circe, i canti delle sirene, i passatempi dei proci, che tentano anzi assediano le caste Penclopi; da una parte, costumi rustici e ruvidi, dall'altra giuochi, vesti magnifiche, cibi squisiti e arti d'intagliare in bassorilievo e fondere in metalli; da una parte, rigida società eroica, dall'altra, perfino, accenni a libertà popolari. Questi costumi cosí delicati mal si convengono con gli altri tanto selvaggi e fieri, che nello stesso tempo si narrano dei medesimi eroi, particolarmente nell'Iliade. Messi insieme tutti a un tempo, riescono incompossibili: dai costumi dell'eta troiana si sbalza senza transizione a quelli lel tempo di Numa; talché, " ne placidis coëant inmitia », si è costretti a pensare che i due poemi furono per piú etia e da piú mani lavorati e condotti. Incoerenze di allusioni geogratiche, che anch'esse trabalzano in ambienti fisici diversi e lontani: l'Ilicule all'oriente della Grecia, verso settentrione; l'Odissea, all'occidente, verso mezzodi. Incoerenze di linguaggio, sconcezze di favellari, che permangono nonostante l'emendazione di Aristareo, e per la quale si sono proposte le piú strane teorie, come quella che Omero sarebbe andato raccogliendo il suo linguaggio la tutte le varie popolazioni greche.

Dai poemi passando alle tradizioni circa il loro autore, nessuna fede meritano le vite di Omero seritte da Erodoto (o da chi altri ne sia l'autore) e da Plutarco (dallo pseudo Piutareo). Intorno a Omero mancano le notizic piú elementari: proprio dove dagli antichi si tratta di questo che fu il maggior lume di Grecia, siamo lasciati affatto al buio. Non si sa di Omero né il tempo in cui visse né il 
luogo di nascita: ciascuno dei popoli di Grecia lo rivendicara suo cittadino. Si narra bensi ch'egli fosse povero e cieco; ma codeste sono di quelle minute particolarita che mettono sospetto, come muove a riso ciò che dice Longino che Omero da giovane componesse l'Iliade e da vecchio l'Odissea. Mirabile che si conoscessero queste private faccende di un uomo del quale s'ignoravano poi due cose da nulla: il tempo e il luogo! E la critica deve domandarsi, anzitutto, come mai fosse possibile clie un sol uomo componesse due cosi lunghi poemi, in un'età nella quale non esisteva ancora la scrittura; giacché le tre iscrizioni eroiche, una di Anfitrione, l'altria d'Ippocoonte e la terza di Laomedonte, delle quali con troppo buona fede parla il Vossio, sono imposture, simili alle tante che sogliono eseguire i falsificatori di medaglie antiche.

Per tutte queste considerazioni sorse nel Vico il sospetto che Omero non fosse, per lo meno in tutto e per tutto, un personaggio reale, ma anch'esso * per la metà» uno di quei caratteri poetici ai quali si erano riportate nell'antichitả lunghe serie di azioni, opere e avvenimenti. Se infatti ci si prova a pensare che i poemi omerici non siano l'invenzione di un individuo, ma due grandi tesori dei costumi della Grecia antichissima, che contengono la storia del diritto naturale e dell'età eroica delle genti greche; se invece che a uno o due poeti síngoli si pensa a un popolo intero poetante; invece che a due opere di getto, a una poesia popolare svoltasi per secoli: tutto si rischiara e si riaccorda. Si spiegano le stravaganze delle favole, perché la composizione dell'Iliade e dell'Odissea appartiene alla terza età di quelle, vere e severe presso i poeti teologi, alterate e corrotte presso gli eroici, e ricevute cosi corrotte nei due poemi. Si spiegano le varieta dei costumi, richiamanti le-varie età della composizione; e altresí l'Omero giorane e l'Omero vecchio, simbolo del piú antico e del 
piú recente tempo della Grecia primitiva. Si spiega la varieta dei luoghi di nascita e di morte, assegnati al loro autore, e le varietà dei suoi linguaggi, perché varî furono i popoli che produssero quei canti. Si spiega, infine, perchè ogni popolo greco volle Omero suo concittadino, per li ragione cioc̀ che essi popoli per l'appunto furono quest'Omero; e perché fosse detto cieco e mendico, perché tali erano di solito i cantori che giravano per le fiere reeitando le storie. Bisogna dunque ehe Omero, perché sia inteso nella sua verita, venga sperduto dentro la folla dei greei popoli e considerato come un'idea o carattere eroico di uomini greci in quanto narravano cantando le loro storie. Cosi quelle che sono sconcezze e. inverisimiglianze nell'Omero finora creduto, diventano nell'Omero qui ritrovato tutte convenevolezze e necessita. E, innanzi tutto, gli si aggiunge una sfolgorantissima lode d'essere stato il primo storico a noi pervenuto dell'intera Grecia. In Omero si ha il documento della primitiva identita di storia e poesia, e una conferma di quel che il Vico credeva di leggere in Strabone, cioè ehe prima di Erodoto, anzi prima di Ecateo milesio, la storia dei popoli di Grecia fu scritta dai loro poeti. Nell'Odissea, volendosi lodare alcuno per avere ben narrata una storia, si dice " averla racentata da musico e da cantore ».

Il Vico non si perde in poco feconde congetture istituendo indagini piú particolari circa il modo di elaborazione dei poemi omerici. Propende tuttavia, come s'è visto, per due prineipali autori-poeti, l'uno per l'Iliade, nativo dell'oriente di Greeia, verso settentrione, l'altro per l'Odissea, nativo dell'occidente ver'so mezzodí; e il nome "Omero » intende come di compositore e legatore di favole. Ma, d'altro canto, a causa degl significato puramente ideale ehe per lui ha quel nome, non è da escludere, forse, l'interpetrazione che $i$ due Omeri fossero, a loro volta, 
due correnti poetiche e due gruppi di popoli o di cantori popolari. Le persone storiche, che egli si trova innanzi, sono i rapsodi, uomini volgari che partitamente, chi uno chi altro, andavano recitando i canti d'Omero nelle fiere e nelle feste per le città greche. Lunga età corse dalla primitiva composizione fino ai Pisistratidi, i quali fecero dividere e disporre i canti omerici nei due gruppi dell'Iliade e dell'Odissea (donde si deduce quanto innanzi dovessero essere stati una confusa congerie di cose), e ordinarono che d'indi in poi fossero cantati dai rapsodi nelle feste panatenaiche.

Comunque, non è di certo in questa risoluzione materialmente intesa dell'individuo Omero in un mito o carattere poetico l'importanza (come, forse, non è la verita) della teoria vichiana. Dalle incoerenze ch'egli non pel primo notava, e non sempre con esattezza (la qual cosa, per altro, è di poco rilievo, essendo agevole compensare le osservazioni inesatte con le molte altre esatte da lui tralasciate), non c'era rigoroso passaggio logico all'affermazione della non esistenza di un Omero individuo, principale autore di uno o di entrambi i poemi. Quelle incoerenze valevano a dimostrare che il poeta o $i$ poeti lavorarono sopra una ricea materia tradizionale, della prove- nienza piú varia per luoghi e per tempi, e non tanto disposta a strati secondo la provenienza (che era a un dipresso l'ipotesi messa innanzi dal D'Aubignac), quanto piuttosto in tutti $i$ suoi strati mescolata e sconvolta. Uno o molti poeti, orvero molti poeti e un abile collettore dei loro canti, o una società di abili collettori; queste e altrettali ipotesi si potevano proporre (come si sono proposte di poi) con pari diritto, e sostenere (come sono state sostenute) con argomentazioni parimente valide e parimente difettose perchè non documentabili. Ma nel fondo di quella risoluzione di Omero in un carattere poetico (come analo- 
gamente in altre simili risoluzioni fatte o tentate dal Vico) era la scoperta della lunga e laboriosa genesi storica attraverso cui era passata la materia di quei poemi, che, in questo senso, ben potevano dirsi prodotto di collaborazione dell'intero popolo greeo. La sostituzione a Omero di un popolo di Omeri fu, anche questa volta, la mitologia tessuta dal Vieo sulla propria scoperta: mitologia che deve essere ritradotta in prosa seientifica. Parimente l'analisi che il Vico svolge del costume dei poemi omerici, oltre che nou del tutto nuova, può ben essere, ed è veramente, non solo qua e la mista di qualche inesattezza, ma, in genere, esagerata e unilaterale. Ciò nonostante, quell'analisi, presa nell'insieme, rappresentava un immenso progresso e apriva nuove vie alla critica. E in qual altro modo si poteva abbattere il resistente fantoccio dell'eroe omerico ingentilito, gran signore e buon principe, esempio imitabile di virtú civili, nilitari e private, se non scagliandogli contro quell'Achille contadinesco, tutto passioni elementari, violento, testardo, irriflessivo, pronto all'impeto generoso ma non meno allo sfogamento bestiale?

Né minore progresso il Vico fit compiere all'intelligenza artistica della poesia omerica: ed è qui anzi la sua maggiole originalità. La riconosciuta mancanza di buona e ragionata filosofia nel poeta Omero sarebbe stata per ogni altro eritico di quel tempo (ed era infatti pel D'Aubignae) l'enunciazione di un grave biasimo; ma in boeca al Vico, e in conseguenza delle sue nuove idee estetiche, suonava lode. Gli errori, che si seorgevano in Omero tl lume dell'intellettualismo, inducevano i critici della scuola neoclassica, "omerofili " ardenti, a ripetere volentieri l'oraziano: "quandoque bonus dormitat Homerus ". Ma il Vico, tutt'all'opposto: " se cosi spesso non avesse dormicchiato, Omero non sarebbe stato cosi buon pocta! » ( nisi ita sape dormitaret, nunquam bonus fuisset Homerus! »): 
appunto perché filosofo non fu, Omero fu poeta grandissimo. Ebbe memoria vigorosa, fantasia robusta, ingegno sublime; onde né filosofie né arti poetiche e critiche, le quali vennero appresso, poterono fare un altro poeta che per corti spazî gli tenesse dietro. Caratteri eroici si seppero fingere solamente da lui; le sue comparazioni sono incomparabili; le sue sentenze s'innalzano, insino al cielo sublimi, e sono individuate in coloro che le sentono, prodotte in forza di un'accesa fantasia; la sua locuzione, piena d'evidenza e di splendore; la sua favella, tutta per somiglianze, immagini, paragoni, priva di quelle idee di generi e di specie che definiscono intellettivamente le cose. Egli non è delicato ma grande, perché la delicatezza è una piccola virtú e la grandczza naturalmente disprezza le cose piccole: anzi (diceva il Vico con uno dei suoi magnifici paragoni tante volte ripetuto di poi), a quel modo che un grande rovinoso torrente non può far di meno di portare seco torbide l'acque c rotolare sassi e tronchi con la violenza del corso, cosí in Omero si trovano sovente detti di cose vili. Ma il torrente corre impetuoso e superbo con tutte le scorie che trascina seco; e Omero, nonostante le sue rozzezze, e in parte a cagione di esse, è pur sempro il padre e il principe di tutti gli altri poeti.

La rinnovazione della critica omerica portava implicita in sé la rinnovazione di tutta la storia letteraria antica. Ma il Vico fa solo pochi accenni ad altri punti di questa storia: egli non era uno specialista (ignorava il greco), non scrisse da specialista, e troppe volte, non potendo risolvere col documento e col pensiero i problemi che si proponeva, li risolse con I'immaginazione, la quale per altro era sempre in lui tutta solcata da lampi di verità. Cosí i poeti ciclici non saranno stati denominati a questo modo dal circolo di genti valgari in mezzo al quale (simili ai " Rinaldi " o cantastorie, che il Vico vedeva sul molo di 
Napoli) declamavano i poemi, e quel circolo non avrì nulla da vedere col « vilem patulumque orbem * oraziano; ma che essi fossero poco diversi dai cantastoric, non è male osservato. Cosi non è il caso d'indugiarsi sulle sue congetture circa l'età di Esiodo o di Esopo, né si prenderanno alla lettera le tre epoche che egli stabilisce della lirica: inni religiosi, canti funerarî per eroi morti, e, infine, lirica melica $o$ * arie per musica s, nella quale ultima rientrerebbe Pindaro, che fiori nei tempi della virtú « pomposa» della Grecia, ammirata nei giuochi olimpici dove tali poeti cantarono; pure, è qui ben avvertito il divario tra la lirica primitiva e quella affinata e dotta. Della tragedia il Vico pone l'origine nel ditirambo o satira drammatica (di cui nessun esempio si era serbato) e in costumanze villeresche che ravvicina a quelle che erano vive ancora ai suoi giorni nella Campania durante la stagione della vendemnia; e ne nota le relazioni con l'epos. Ia tragedia nacque rozza, quando gia era spento lo spirito eroico, e si perfezionò affiatandosi con la poesia omerica, ispirandosi ai personaggi di questa e rifuggendo da caratteri di nuova invenzione. Strettamente imparentata con la tragedia fu la commedia antica, derivata anch'essa da un coro e serbante la sua impronta arcaica in quel porre sulla scena personaggi viventi e fatti reali. Un profondo rivolgimento di spirito segnò, invece, la comnedia nuova, dove la filosofia fece sentire direttamente la propria efficacia. Ai generi fantastici erano succeduti gl'intelligibili e ragionati; e Menandro e gli altri poeti della nuova commedia, vissuti ai tempi umanissimi della Grecia, presero i generi intelligibili dei costumi umani e li portarono in ritratti nelle loro commedie, sulle quali si sente che è passato il soffio della filosofia socratica. I personaggi della nuova commedia furono perciò finti di getto, e non piú personaggi pubblici ma privati; e, poiché il coro è un pubblico che ragiona 
e di non altro che di cose pubbliche, in quella commedia non poté piú avere luogo il coro. Si cominciarono, circa quel tempo, a introdurre nella poesia gli eroi ottimi, gli eroi moralmente perfetti. Aristotele, memore della forte individuazione dei caratteri omerici, ancora alzava in precetto d'arte poetica che gli eroi di tragedia non siano né ottimi nẻ pessimi, si bene mescolati di grandi virtú e grandi vizî. Ma i poeti dei tempi tardi foggiarono un " eroismo di virtú », giovandosi dell'idea costruita dai filosofi: un eroismo che pừ chiamarsi, perfino, "galante ". Essi o finsero perciò favole nuove di pianta, o le favole, nate dapprima gravi e severe quali convenivano a fondatori di nazioni, effeminarono piegandole all'effeminarsi dei costumi. Galante è altresi il «pastorcccio » dei bucolici greci, dei Mosco e degli Anacreonte, « marci di amore delicatissimo ». Un'osservazione generale intorno alla letteratura greca e romana è che in esse furono con tanto rigore guardati reciprocamente i confini della poesia e della prosa che nessun antico scrisse orazioni e poemi insieme (unica eccezione forse, i cattivi versi, ridenda poëmata, di Cicerone); il che il Vico tentava di spiegare coi costumi denocratici che costringevano gli oratori a tenersi studiosamente lontani dal culto di modi d'espressione alti e immaginosi, poco intelligibili al pupolo e perciò d'impedimento all'agevole e piena notizia delle cause da giudicare.

Della letteratura romana il Vico non discorre con la larghezza usata per la greca, la quale gli porgeva documenti ben piú primitivi. Intravvide, per altro, negli inizî di essa, qualcosa di analogo alla letteratura greca : i primi poeti c i primi autori della lingua latina furono i Salî, poeti sacri; e ciò convencrolmente ai principî dell' umanitì delle nazioni, le quali nei primi tempi, che furono religiosi, non dovettero altro lodarc che gli dèi. Fa al modo stesso che quelle piủ antiche memorie a noi giunte della lingua latina, 
ossia i frantumi degl'inni saliari, hanno un'aria di verso eroico, il medesimo metro si sente nelle memorie dei trionfatori romani, come nel «Duello magno dirimendo, regibus subigendis »di Lucio Emilio Regillo, e nel « Fundit, fugat, prosternit maximas legiones » di Acilio Glabrione. Anche i primi poeti romani cantarono storie vere, come Livio Andronico (diceva il Vico, seguendo un errore di Celio Rodigino) la Romanide, contenente gli annali degli antichi romani, e Nevio e poi Ennio le guerre cartaginesi; e la satira sparlava di persone reali e per lo piú notorie. Senonché, diversamente dai greei, i romani, i quali nei loro costumi camminarono con giusto passo, non fecero rapido e brusco passaggio dalla barbarie alla delicatezza, epperò perderono affatto di veduta la loro storia degli dèi (che Varrone chiama « tempo oscuro » dei romani), e conservarono quindi in fivella volgare solamente la storia croica, che si stende da Romolo fino alle leggi Publilia e Petelia. La letteratura romana, nei suoi maggiori monumenti, è opera di poeti colti, quale fu Virgilio, che il Vico ammira per la profonda scienza delle antichita eroiche, ma di eui dice (in contrasto con la critica neoclassica e, come a lui pareva, in accordo col sentimento di Plutarco e di Longino) che, in quanto a forza poetica, non si può metterlo neppure a confronto con Omero. Di Lucrezio giudica che portò in linyna latina, e per dippiú in versi, un'affatto nuova materia greea, ma, a riserva delle poetiche introduzioni e d'una o altra digressione (come quella della delicata inimitabile descrizione della tenera giovenca che ha perduto la madre, e quella, incomparabile nel genere grande, ove descrive la pestilenza di Atene), del rimancute tratta le materie fisiche con istile niente diverso da quello con eui si sarebbero insegnate in una scuola latina di filosofia naturale: laddove Virgilio, nella Georgica, tratta dell'arte vilIercecia «poeticamente». Poesia colta e riflessa è altresi 
in Orazio, il quale, come Pindaro nei tempi pomposi della Grecia, lavorò le sue odi in quelli più «sfoggiosi » di Roma, cioè ai tempi di Augusto.

Una letteratura che gli avrebbe offerto elementi preziosi per lo studio della poesia primitiva, sarebbe stata quella biblica; e il Vico muove in verita qualche passo per avvicinarlesi, quando nota che la poesia fu la lingua primitiva di tutte le nazioni, «anche dell'ebrea »; che la lingua ebraica omnis poëtica est parabolis ac similitudinibus referta; e che Mosè "non f'ece nessun uso della sapienza riposta dei sacerdoti egiziani » e tessé la sua storia « con parlari che hanno molto di conformità con quelli di Omero, e spesso li vince nella sublimita dell'espressione ». Ma subito se ne discosta, quasi un istinto lo avverta che gli poteva accadere di trattare il Pentateuco come l'Iliade e Mosè come Omero. Onde gli accade di estasiarsi innanzi a quel motto con cui Dio si descrive a Mosè: "Ego sum qui sum», che è di una profondità metafisica (egli dice) raggiunta dai greci appena con Platone, il quale concepí Dio come tó ör, e ignorata fino a tempi tardissimi dai latini; tanto che la voce « ens $»$ non è latina pura ma della bassa latinita. Ovvero insiste perché si osservi che nei tempi nei quali correva tra i greci un diritto naturale tutto superstizione e fierezza, Iddio dette al suo popolo una legge « si ripiena di dignita circa i dogmi della divinità e sí ricolma di umanità circa le pratiche della giustizia, che neppure negli umanissimi tempi della Grecia l'intesero i Platoni o la praticarono gli Aristidi »: una legge « i cui sommi dieci capi contengono un giusto eterno ed universale sull'idea ottima dell'umana natura schiarita, che formano per abiti un tal sapiente che difficilmente per råiocinî potrebbono le massime delle migliori filosofie; onde Teofrasto chiamò gli ebrei filosofi per natura». La « volonta di credere» sarebbe qui tanto piú efficacemente operante, e tanto piú manifesta, se, come 
sospettiamo, il Vico aveva letto il Tractatus theologicopoliticus del reprobo Spinoza; dove ai profeti ebrei si negano le «sublimes cogitationes», riconoscendo loro la sola "pietatem"; si sostiene che non insegnarono se non cose "admodum simplices qua ab unoquoque facile percipi poterant, atque has eo stilo adornavisse iisque rationibus confirmavisse quibus maxime multitudinis animus ad devotionem erga Deum moveri posset »; si afferma che le leggi rivelate da Dio a Mosè « nihil aliud fuissent quam iura singularis hebreorum imperii »; e, andando ben oltre gli sparsi accenni fatti da altri critici, s'istituiscono spregiudicate indagini sul testo della Bibbia e sull'autenticità e la diversitit di autori dei libri del Pentateuco. Si direbbe quasi che dalla critica biblica dello Spinoza il Vico avesse avuto incentivo alla sua della formazione e dello spirito dei poemi omeriei, e che, passato per tal modo dalla storia sacra alla profana, da Mosè a Omero, si fosse poi ostinato a non ripassare a niun patto da Omero a Mosè, dalla storia profana alla sacra. 



\section{XVII}

\section{LA STORIA DI ROMA}

E LA FORMAZIONE DELLE DEMOCRAZIE

\section{L}

a societí eroica, nel periodo di fresea origine e di vigore nol quale l'abbiamo descritta, contiene e comprime in sé encrgicamente, e ne fa suo proprio sostegno, l'elemento di opposizione, i famoli o clienti o vassalli: la plebe. Ia questo elemento ricsee a poco a poco a distacearsi, a eontrapporsi, a lottare apertamente e ininterrottamente, in modo da sconvolgere via via quella vecchia società e dare vita e forma a una nuova, nella quale esso stesso si risolve: la società democratiea, la repubblica popolare. Il Vico intende anche questo processo come uniforme in tutti $\mathbf{i}$ popoli; ma poiché le sue riferenze ad altre storie, che non siano quella di Roma, maneano o sono vaghissime (appena fa qualche aceenno all'origine della democrazia ateniese), la descrizione di quel proeesso diventa, nelle pagine della Scienza nuova, un brano della storia romana, o, eome ora si direbbe, della storia sociale di Roma.

Quel che il Vico poté eongetturare cirea le popolazioni e la primitiva civilta d'Italia, non ha molta importanza, perehé in questo campo, piuttosto areheologieo ed etnografico che storico, egli non condusse studî particolari. Nel De antiquissima italorum sapientia aveva dato all'origine 
di Roma lo sfondo di un'antichissima cultura italica, anteriore alla greca e proveniente dall'Egitto, che i romani si sarebbero appropriata in modo conforme all'indole loro, rigettando cioè i presupposti tcorici e prendendo i risultati pratici (dagli etruschi la tragica religione e l'arte di schierare in battaglia, come poi dagli ateniesi e dagli spartani le leggi), e serbando per tal modo l'ignoranza e la ferocia; onde sarebbe provenuto che essi parlassero lingua di filosofi senza essere filosofi. In séguito, il Vico ritenne ancora per qualche tempo l'anteriorità e l'indipendenza della piú antica civiltà italica rispetto alla greca, e considerò Pitagora, piuttosto che fondatore, cultore d'italica sapienza; ma anche questa tesi sembra, in ultimo, da lui abbandonata, come definitivamente abbandonò quella circa l'origine della religione, della lingua, dei costumi e delle leggi di Roma da imitazioni staniere, "ingenuamente professando di avere per questa parte errato » sull'ésempio del Cratilo platonico. Tra quali condizioni propriamente Roma nascesse, il Vico non sa dire; certo è per lui che, se con la storia di Roma non comincia il mondo, quella storia è per altro un nuovo cominciamento. Il punto di partenza che egli assume è l'asilo di Romolo, cioè le famiglie dei padri che accolgono nel loro seno i divaganti e ne fanno i loro famoli. Niente colonia troiana; il Vico conosce la Lettre del Bochart al De Segrais (1663) contro la leggenda della venuta di Enea in Italia, e ne accetta le conclusioni, confermanti i dubbî già manifestati da alcuni storici antichi. Per lui, l'origine troiana è una favola, nata dall'incrocio di due diverse «borie " nazionali: quella dei greci, che fecero tanto rumore della guerra di Troia e intrusero in Roma il loro Enea, allorché di Roma presero a narrare la storia, e quella dei romani, che per vantare famosa origine straniera lo ricevettero; favola, dunque, che non poté formarsi se non molto dopo í tempi della guerra con Pirro (all'in- 
circa a quelli di Fabio Pittore e Cincio Alimento), quando i romani cominciarono a dilettarsi delle cose greche. Piuttosto, a spiegare i nomi e i miti greci misti al racconto della primitiva storia di Roma, e l'alfabeto romano tanto simile a quello greco antico, egli sarebbe incline all' ipotesi che i romani avessero, nei loro primi tempi, vinta e distrutta nel lido del Lazio alcuna colonia greca (rimasta poi sepolta nelle tenebre dell'antichita), e, con l'accoglierne in Roma come rifuggiti e socî gli abitatori, si fossero imbevuti di non poche tradizioni e costumanze elleniche.

Neppure s'indugia il Vico sugl'incidenti storici del periodo regio; anzi, in ciò è una delle principali differenze tra la sua critica e quella che era cominciata a esercitarsi, o si esercito poco dopo di lui, intorno ai primi secoli della storia romana. Il Vico non mira a porre in luogo di aneddoti leggendarî aneddoti storici, ma a intendere la sostanza delle istituzioni e $\mathrm{i}$ modi del loro cangiamento. Due idee direttive (come si è visto) egli ha circa il periodo regio: l'una, che questo non fu periodo di monarchia ma di aristocrazia, e che sia da interpetrare conforme al tipo della società eroica o della repubblica dei padri; l'altra, che i nomi dei re stiano come simboli o " caratteri poetici.» delle istituzioni di quella societa, e talvolta anche, per « anacronismo ", si raccolgano sotto di essi avvenimenti e riforme dei secoli susseguenti. Come anche si è avuto occasione di accennare, l'ordinamento di Servio Tullio non era, a giudizio del Vico, da fraintendere, al modo che fecero i romani in tempi seriori, quale pianta della liberta popolare, perché fu in effetto pianta della libertà signorile, per la quale i patrizî concessero ai plebei il dominio bonitario dei campi con l'obbligo di pagar loro, e cioè ai singoli patrizî, il censo e di servirli a proprie spese nelle guerre. E Giunio Bruto, discacciando i Tarquinî e surrogando il re con due consoli o re aristocratici annuali, restitui la repubblica ro- 
mana ai suoi principî, ossia riordinò la libertà dei signori dai loro tiranni, non già la libertà del popolo dai signori.

L'oppressione che i patrizî escrcitarono sulla plebe dopo la loro reintegrazione compiuta da Giunio Bruto, la lotta che essi suscitarono e la resistenza che spiegarono, furono l'anima del nuovo svolgimento, e contengono il segreto della grandezza di Roma, la * clavis historia romana unicersce». Polibio offerse di questa grandezza una spiegazione troppo vaga col riporla nella virtú o nella religione dei patrizî e narrò piuttosto i fatti di essa virtú che non le loro cagioni. Il Machiavelli anche è censurato dal Vico, una volta perché riferí la causa della grandezza romana a sparsi istituti romani civili e militari senza scoprire la fonte di quegl'istituti, cioè la natura di quella società; e un'altra, perché ne arrecò una causa parziale, la magnanimità della plebe. Plutarco gli sembra inferiore a tutti, perché, invidioso della virtú e sapienza romana, attribuí quella grandezza alla fortuna. Ma, in verità, i romani si sottomisero le altre città del Lazio, poi l'Italia e in ultimo il mondo, perché ebbero giovane l'eroismo, quando tra gli altri popoli del Lazio cominciava a invechiare. Per quel vigore giovanile, i patrizî furono forti nel custodire il loro ordine e la religione su cui si fondava e che lo garantiva (i nobili, osserva il Vico, cosí, sempre e dappertutto, si comportano verso la religione nativa, e quando prendono a disprezzarla è gran segno che una nazione vada a perdersi); la plebe fu magnanima nel volere comunicati a sé la religione, gli auspicî e tutte le ragioni civili; i giureconsulti, infine, furono sapienti nell' interpetrare e condurre le antiche leggi fil filo ai nuovi casi che chiedevano di essere regolati, e si studiarono a tutto lor potere che quanto meno e con tardi passi si estendessero le pirole di quelle. Per tal cagione principalmente l'impero romano cotanto s'ingrandí e durò, perché nclle sue vi- 
cende politiche procurò di stare fermo sopra i suoi principî. Alle gare tra gli ordini si deve altresi la prodezza in guerra, perché i nobili naturalmente si consacravano per la salvezza della loro patria, con la quale avevano salvi tutti gli onori civili dentro il loro ordine, e i plebei compievano segnalatissime imprese per dimostrarsi meritevoli degli onori dei nobili. E quando i romani stesero le conquiste e spiegarono le vittorie sul mondo intero, essi fecero uso di quattro norme, che gia avevano praticato coi plebei dentro Roma: perché le provincie feroci ridussero in clientele mandandovi colonie, alle mansuete rilasciarono il dominio bonitario dei campi, all' Italia permisero il dominio quiritario, ai municipî o città benemerite largirono lo stesso trattamento di parificazione che aveva ottenuto, in ultimo, la plebe.

I risultati delle prime lotte, nelle quali, secondo il Vico, era in questione il dominio bonitario dei campi (riconosciuto gia nella costituzione serviana, ma ritolto dai nobili pei (lebiti dei censi non soddisfatti), condussero al tribunato, e, per la richiesta che facevano i plebei del dominio quiritario, alla legge delle dodici tavole, che accolse quella conquista plebea. Ma la legge delle dodici tavole fu, insieme, la conquista del diritto scritto, la cessazione dell'arcano onde i patrizî avvolgevano le leggi, che essi soli conoscevano, intendevano e interpetravano, e perciò amministravano a loro arbitrio. A questa divulgazione e fissamento del diritto scritto i patrizì non poterono bonariamente accondiscendere con quel « desideria plebis non aspernari », che dice Livio; ma piuttosto vi dovettero repugnare con quella ritrosia di cui narra Dionigi d'Alicarnasso, espressa dal « mores patrios servandos, leges ferri non oportere».

La storiografia posteriore adornò di favole l'origine della legge delle dodici tavole; e raccontò, tra l'altro, 
della missione che $\mathrm{i}$ decemviri avrebbero inviato ad Atene per riportarne la nuova legge: cosa affermata da Livio e da Dionigi, ma ignorata da Polibio e che il Vico con gran travaglio si sforzava di dimostrare non creduta da Varrone e da Cicerone. Come mai nella natura selvaggia e ritirata delle prime nazioni, tra le quali non poté formarsi comunicazione di lingue se non dopo sorte le occasioni di guerre, alleanze e commerci, si sarebbe da oltremare, dalla lontana Attica, tragittata in Roma la fama della sapienza di Solone? Come i romani di quei tempi arrebbero avuto notizia tanto esatta circa la qualità delle leggi ateniesi da stimarle proporzionate a sedare le contese accese tra i loro plebei e i loro nobili? Quali ambasccrie potevano correre allora tra i greci e quei romani che, ancora parecchi anni dopo, erano maltrattati come sconosciuti dai greci di Taranto? E che cosa dire poi di quegli ambasciatori che se ne tornarono da Atene con le leggi greche nelle valigie senza conoscere che cosa per l'appunto contenessero, talché se per caso non si fosse trovato in Roma lo scolaro di Eraclito, Ermodoro, bandito dalla sua patria, i romani non avrebbero saputo che farsi di quel chiuso e inattingibile tesoro? Ed Ermodoro come fece mai a tradurle con tanta latina purità che Diodoro siculo giudica non odorare nulla affatto di grecismo, in guisa cosi perfetta che nessuno scrittore latino seppe piú in nessun tempo raggiungere nel tradurre dal greco? Come rivestí idee greche con voci latine tanto propric (per es., auctoritas), che i greei affermano non esservi in lingua greca termini corrispondenti per ispiegarle? Ma la lettera di Eraclito a Ermodoro dovette essere mandata per quelle stesse poste, per le quali Pitagora aveva fatto i suoi lontanissimi viaggi per il mondo, e, insomma, è da ritenere un' impostura bella e buona; impostura la venuta stessa di Ermodoro in Italia, ché non mai un cittadino della libera e possente Efeso si sarebbe 
recato, per trovar conforto all'esilio, nella barbarica Roma, a tutti ignota fuori dei confini del Lazio; impostura la statua di Ermodoro, che Plinio narra vedersi ancora ai suoi tempi nel Comizio, nel modo in cui ora a Napoli si vedono la grotta della Sibilla e la tomba di Virgilio; e, insomma, tutta la favola della origine ateniese di quelle leggi è una boria di dotti, che prima le fecero provenire da altri popoli del Lazio (per es., dagli equicoli); poi dalle citta greche d'Italia, poi da Sparta, e finalmente da Atene, sul qual nome, per la fama dei filosofi ateniesi, si fermarono soddisfatti. Di certo le leggi delle dodici tavole presentano somiglianze non solo con le leggi ateniesi o spartane, ma anche con quelle di altri popoli e con le mosaiche; ma la ragione di ciò è nell'uniforme corso delle nazioni. Di certo, ai decemviri furono nell'antichità attribuite leggi con evidenti tracce greche, come quella che proibisce il lusso greco dei funerali; ma questo accadde perché, come si è notato, la legislazione decemvirale, non meno che i nomi dei singoli re, divenne un « carattere poetico », e a essa si rapportarono tutte le leggi che dipoi agguagliarono la libertà e che si scrissero in pubblici monumenti. Ma la legge originaria delle dodici tavole, tanto incivile, rozza, inumana, crudele e fiera, tanto poco conveniente al tempo in cui Atene sfoggiava di cultura, è un gran testimonio dell'antico diritto naturale delle genti del Lazio, dei costumi che si erano cominciati a celebrare colà tin dall'età di Saturno.

Ottenuto il dominio quiritario dei campi e la legge seritta, la lotta ricominciò pel diritto dei connubî: lotta di cui si perse il vero significato e sulla quale gli stessi stolici antichi scrissero cose assurde, inmaginando che essa fosse impiantata sulla richiesta dei plebei (i quali erano poco piú che miseri c vilissimi schiavi) di potersi liberamente imparentare coi nobili. In tal guisa la storia romana 
diventa assai piú incredibile della favolosa dei greci, perché di questa non si sa che cosa abbia voluto dire, ma quella si oppone a tutto l'ordine dei desiderî umani, presentando una plebe che prima aspira alla nobiltà, poi agli onori e magistrature, e in ultimo alla ricchezza; laddove gli uomini prima desiderano ricchezze, poi ufticî di stato, e in ultimo nobiltà. Senonché la plebe romana non bramava già « connubia cum patribus », ma « connubia patrum»; non il diritto d'imparentarsi coi nobili (cosa che non arrebbe ardito pretendere e che in fondo non le premeya), ma il diritto di contrarre nozze solenni, come le contraevano i nobili. Perché, senza le nozze solenni, senza gli auspicî, i plebei non potevano esercitare in effetto il diritto quiritario dei campi, e non potevano tramandarlo ai loro congiunti, essendo privi di suità, di agnazione e di gentilità. La richiesta dei connubî valeva, insomma, né piú né meno che quella della cittadinanza; e fu soddisfatta con la rogazione Canuleia.

Dopo la quale, i plebei avanzarono la richiesta delle dipendenze di ragion pubblica; e prima ne riportarono gl'imperî coi consolati, e finalmente i sacerdozî e i ponteficati, e con questi insieme la scienza delle leggi. In tal modo la pianta di libertá signorile, tracciata da Servio Tullio, si cangiò in pianta di libertà popolare; e il censo, che si pagava ai patrizî, fu pagato invece all'crario, che somministrava ai plebei le spese nelle guerre. La difficolta concernente il cosiddetto censo di Servio Tullio fu detta dal Vico « la piú aspra sua meditazione sulle cose romane », con la quale riuscí a risolvere l'anacronismo del censo serviano e a riportare la riforma al tempo di Fabio Massimo. I tribuni procedettero a chiedere la potestà di comandare le leggi, perché le leggi precedenti, Orazia e Ortensia, non avevano dato ai plebisciti forza di obbligare il popolo tutto se non nei due casi particolari, a cagion dei quali la 
plebe si era ritirata una volta sull'Aventino e un'altra sul Gianicolo. Questa nuova conquista, che stabili la superiorità della plebe c mutò la repubblica da aristocratica in popolare, fu la legge Publilia, ordinata dal dittatore $\mathrm{Pu}$ blilio Filone, per la quale i plebisciti * omnes quivites tenevent ". L'autorità del senato ne venne ristretta, perché laddove, precedentemente, di quel che il popolo aveva deliberato i padri si facevano "auctores ", ora i padri erano essi " autori " al popolo, che approvava le leggi secondo la formola proposta dal senato, o le antiquava, cioè dichiarava di non volere novita. La plebe ottenme, inoltre, l'ultima magistratura ancora non comunicata, la censura. La legge Petelia, che seguí pochi anni dopo, cancello l'ultimo vestigio di legame feudale, il nesso (nexus), che rendeva i plebei, per causa di debiti, vassalli ligi dei nobili e li costringeva sovente a lavorare tutta la vita nelle private prigioni di costoro.

Quando Fabio Massimo alla divisione tra patriziato e plebe, coi corrispondenti comizî curiati e tributi, ebbe sostituita la divisione secondo i patrimonî dei cittadini, ripartiti nelle tre classi di senatori, cavalieri c plebei, l'ordine dei nobili venne a sparire affatto, e "senatore»e «caraliere» non furono píu sinonimi di «patrizio», né "plebeo»d' «ignobile ». Ma al senato rimase il dominio sorrano sopra i fondi del romano imperio, che era gia passato nel popolo; e, merce i cosi detti senatoconsulti ultimi o ultima necessitatis", lo mantenne con la forza delle armi finché la romana fu repubblica popolare; e quante volte il popolo tentì di disporne, tante il senato armò i consoli, i quali dichiararono ribelli e uecisero i tribuni della plebe che arevano promosso quei tentativi. Il che si spiega con tua ragione di feudi sovrani soggetti a maggiore sovranita, come al Vico pareva confermato dal detto di Scipione Nasica nell'armare il popolo contro Ti- 
berio Gracco: «Qui rempublicam salvam velit, consulem sequatur ». Aperta con le leggi la porta degli onori alla moltitudine che comanda nelle repubbliche popolari, non restava altro in tempo di pace che contendere di potenza, non con le leggi ma con le armi; e mercé atti di potenza comandare leggi per arricchire, quali furono le agraric dei Gracchi, onde provennero in pari tempo guerre civili in casa e ingiuste fuori.

Tutta la societa, col trionfo della plebe e con la mutazione dello stato da aristocratico in popolare, muta fisonomia. Muta, in primo luogo, la fisonomia della famiglia: nella quale, durante l'impero del patriziato, per serbare le ricchezze dentro l'ordine, solo tardi furono ammesse le successioni testamentarie e facilmente i testamenti venivano annullati; dalla successione paterna era escluso il figliuolo emancipato; l'emancipazione aveva l'effetto di una pena; le legittimazioni non erano perınesse; è da dubitare che le donne succedessero. Ma nella società democratica, poiché la plebe pone tutta la sua ricchezza, tutta la sua forza e potenza nella moltitudine dei figliuoli, si comincia a sentire la tenerezza del sangne, e i pretori ne considerano i diritti e prendono a fargli ragione con le * bonorum possessiones » e a sanare coi loro rimedî i vizî o i difetti dei testamenti, agevolando cosí la divulgazione delle ricchezze, che sole sono ammirate presso il volgo. - Muta il significato degli istituti della proprietà : il dominio civile non è piú di ragion pubblica e si disperde per tutti i dominî privati dei cittadini, che formano ora la città popolare; il dominio ottimo non è piú quello fortissimo, non infievolito da niun peso reale, neppure pubblico, e significa semplicemente quello che sia libero da ogni peso privato: il quiritario non è piú il dominio di cui il nobile era signore feudale e che doveva venire a difendere nel caso che ne fosse decaduto il cliente o plebeo, ma è di- 
ventato dominio civile privato, assistito da rivendicazioni, diversamente dal bonitario che si mantiene col solo possesso. - Le forme dei processi, cosi frondose di finzioni, di formole solenni, di atti simbolici, sono semplificate e razionalizzate: si comincia a far uso dell'intelletto, ossia della mente del legislatore, e i cittadini si conformano in un'idea di comune ragionevole utilita, intesa come spirituale di sua natura. Le caussce, che prima erano formole cautelate di proprie e precise parole, diventano affari o negozî, che si solennizzano coi patti convenuti e, nei trasferimenti di dominio, con la tradizione naturale; e solamente nei contratti che si dicono compiersi con le parole, nei contratti verbali, cioè nelle stipulazioni, le cautele rimangono « caussa », nell'antica proprieta di questo termine. Cosi il certo delle leggi, essendosi la ragione umana spiegata tutta, mette capo nel vero delle idee, determinate con la ragione delle circostanze dei fatti, che è \& una formola informe di ogni forma particolare s (formula natura, come dice Varrone), ehe a guisa di luce informa di sé, in tutte le ultime minutissime parti della superficie loro, i corpi opachi dei fatti sopra i quali ella è diffusa. Nelle repubbliche popolari regnia l'aquum bonum, l'equita naturale. - Le crudelissime pene, che si usavano nel tempo delle monarchie familiari e delle società eroiche (le leggi delle dodici tavole condannavano a essere bruciati vivi coloro che avevano dato fuoco alle biade altrui, precipitati giú dalla rupe Tarpea i falsi testimoni, fatti vivi in brani i debitori falliti), vengono sostituite da pene benigne, perché la moltitudine, che è composta di deboli, è di sua natura incline a compassione. - Le leggi, che erano nelle aristocrazie poche, ferme e religiosamente osservate, si moltiplicano nelle democrazie e si fanno cangevoli e flessibili. Gli spartani, che scrbarono l'aristocrazia, dicevano che in Atene si scrivevano molte leggi, ma le poche 
che erano in Isparta si osservavano: la plebe romana, a guisa dell'ateniese, comandava tuttodí leggi singolari, e invano Silla, capoparte dei nobili, cercỏ di ripararvi alquanto con le «questioni perpetue », perché, dopo di lui, si moltiplicarono di nuovo. - Le stesse guerre, crudelissime nelle repubbliche aristocratiche, che distruggevano le citta conquistate e riducevano $\mathrm{i}$ vinti in gruppi di giornalieri sparsi per le campagne a coltivare a pro dei vincitori, si mitigano nelle repubbliche popolari, le quali, togliendo ai vinti il diritto delle genti eroiche, lasciano loro quello naturale delle genti umane. Gl'imperî si dilatano, perché le repubbliche popolari valgono assai piú delle aristocratiche per le conquiste, e piú ancora vi valgono le monarchie.

Eppure, in questo generale umanarsi dei costumi, scema la sapienza di governo, la virtú politica. Gli antichi patrizî facevano duramente rispettare le leggi; e, avendo privatamente ciascuno gran parte della pubblica utilità, a questo grande interesse particolare, che veniva loro conservato dalla repubblica, posponevano gl'interessi privati minori, e perciò magnanimamente difendevano il bene dello stato e saggiamente consigliavano intorno ad esso. Per contrario, negli stati popolari, e perché i cittadini comandano il bene pubblico che si ripartisce loro in minutissime parti quanti sono essi i cittadini che compongono il popolo, e per le cagioni che producono siffatta forma di stati, che sono affetto d'agi, tenerezza di figliuoli, amore di donna e desiderio di vita, gli uomini sono portati ad attendere alle ultime cireostanze dei fatti che promuovono le loro private utilita, e percio all'equobono, che è ciò solo di cui le moltitudini sono capaci.

A cotal punto balza spontanea, perehé di lunga mano preparata e resa necessaria, la monarchia: quella monarehia che gli ordinarî scrittori di politica facerano venir 
fuori, senza il precorso di tante e sí varie cagioni che debbono condizionarla, di un tratto, al bel principio della storia umana, "cosí come (dice il Vico) nasce, piovendo l'està, una ranocchia ». E molto meno sorse artificialmente, per effetto della favoleggiata legge regia dell" "ignorante grecuzzo "Triboniano, con la quale il popolo romano si sarebbe spogliato del suo sovrano e libero imperio per conferirlo a Ottavio Augusto. La legge, che le die' vita, fu una legge naturale, concepita con questa formola di eterna utilità : che poiché nelle repubbliche popolari tutti guardano ai loro privati interessi ai quali fanno servirc le pubbliche armi in eccidio della propria nazione, per impedire che le nazioni vadano in rovina debba sorgere un solo, come tra i romani Augusto ("qui"; come scrive Tacito, " cuncta bellis civilibus fessa nomine principis sub imperium accepit "); un solo, che con la forza delle armi richiami a sé tutte le cure pubbliche c lasci ai soggetti l'attendere alle loro cose private o a quel tanto delle cose pubbliclie che viene Ioro permesso, e si circondi di pochi sapienti di stato per consultare con l'equita civile nei gabinetti circa i pubblici affari. Quel solo è invocato alla pari da nobili e da plebei: dai nobili, che dopo essere stati abbassati e sottomessi al governo plebeo, abbandonata l'antica aristocratica volontà d'impero, non pensano se non ad avere salva almeno la vita comoda; e dai plebei, che dopo avere sperimentato l'anarchia o la sfrenata demagogia (della quale non si da tirannide peggiore, essendo tanti i tiranni quanti sono gli audaci e dissoluti delle citta), fatti accorti dai proprî mali, chiedono pace e protezione.

La monarchia è, dunque, una nuova forma del governo popolarc. Perché un potente diventi sovrano, è necessario che il popolo parteggi per lui, ed egli deve governare popolarmente, agguagliare tutti i soggetti, umiliare i grandi per tenere libera e sicura la moltitudine dalla Ioro oppres- 
sione, mantenere il popolo soddisfatto e contento eirca il sostentamento che gli bisogna per la vita e circa gli usi della libertà naturale, e adoprare un ben ponderato sistema di concessioni e privilegi o a interi ordini (nel qual caso si chiamano "privilegi di liberta »), o a persone particolåri, promovendo fuori d'ordine uomini di merito straordinario e di virtú eecezionali.

Nella monarchia, che è governo « umano s al pari della democrazia, prosegue e s'intensifica quel processo di mmanamento o ingentilimento dei costumi e delle leggi, che le repubbliche popolari avevano iniziato. Si sciolgono sempre piú i rigidi vincoli della famiglia paterna e gentilizia. Gl'imperatori, ai quali faceva ombra lo splendore della nobiltà, si diedero a promuovere le ragioni della natura umana, comune a nobili e a plebei; e Augusto attese a proteggere i fedecommessi, coi quali nei tempi innanzi, mercé la puntualita degli eredi gravati, i beni erano passati agl'incapaci di eredita, e li trasformò in neessita di ragione, costringendo gli eredi a mandarli ad effetto. Suecesse una folla di senatoconsulti, coi quali i cognati entrarono nell'ol'dine degli agnati; finché Giustiniano tolse le differenze tra legati e fedecommessi, confuse la quarta falcidia e trebellianica, distinse poco i testamenti dai codicilli c adeguò «ab intestato », in tutto e per tutto, gli agnati e i cognati. Tanto le leggi romane ultime si profusero in favorire $\mathrm{i}$ testamenti che, laddove anticamente per ogni leggiero motivo essi erano invalidati, poi si dovettero interpetrare nel modo che meglio conduceva a mantenerli saldi. Caduto affatto il diritto « ciclopico », che i padri avevano esercitato sulle persone dei figliuoli, andò cadendo altresí quello economico sugli aequisti dei tigliuoli; onde gl'imperatori introdussero prima il peculio eastrense per attrarre i giovani alla guerra, poi il quasicastrense per invitarli alla milizia palatina, e finalmente, per tenere con- 
tenti quelli che non erano né soldati né letterati, il peculio arventizio. Tolsero l'effetto della patria potestà alle adozioni, le quali non si contennero piú ristrette nella cerchia di pochi congiunti; approvarono universalmente le arrogazioni, difficili alquanto perché è difficile che un paterfamilias, un sui iuris, si sottometta alla patria potestà d'un estraneo; reputarono le emancipazioni quali benefizî e dettero alle legittimazioni \& per subsequens matrimonium » tutto il vigore delle nozze solenni. L' "impero paterno ", nome superbo che sembrava scemare la maesta imperiale, fu mutato nell'altro di « patria potestà ». L'umanitarismo della monarchia si estese anche a quella parte dell'antica famiglia che erano gli schiavi, avendo gl'imperatori raffrenata la crudeltà dei signori verso di essi e, per favorirli, ampliate negli effetti e ristrette nelle solennita le manomissioni; e la cittadinanza, che prima non si dava se non ai grandi stranieri benemeriti del popolo romano, fu concessa a ognuno che, anche di padre schiavo, purché di madre libera o affranchita, naseesse in Roma. Le pene si addolcirono ancora, e i monarchi si adornarono del grazioso titolo di «clementi ". Le parole delle leggi furono sempre piú largamente interpetrate mercé l'equitì naturale, e si può dire che Costantino cancellasse affatto le formole, quando stabili che ogni motivo particolare di equita faceva mancare le leggi. Si giunse all'estremo opposto del "privilegia ne irroganto » delle dodici tavole; e privilegi erano tutte le eccezioni fatte alle leggi e dettate dal particolare merito dei fatti, che li traeva fuori dalle comuni disposizioni. La restrizione dei diritti ai singoli popoli fu via via abolita: sotto Caracalla si fece di tutto il mondo romano una sola Roma, perché è voto proprio dei grandi monarehi di fare una citta sola di tutto il mondo, secondo il pensiero che Alessandro Magno espresse aicendo che tutto il mondo era per lui una citta, della 
quale la sua falange formava la ròcca. Dagli editti dei pretori si passò, sotto Adriano, all'editto perpetuo compilato da Salvio Giuliano e composto quasi tutto di editti provinciali.

Con la monarchia, il diritto naturale delle genti cede il posto al diritto naturale delle nazioni; onde quella forma politica, sociale e giuridica è la piú conveniente all'umana natura della piú spiegata ragione. Con essa (come si è avuto occasione di accennare) si raggiunge da capo, dopo lungo svolgimento, quell'uno, che era delle monarchie familiari dei primi padri; e il corso delle nazioni si deve considerare affatto compiuto. Andare piú oltre non è possibile: possibile è soltanto, in quella somma delicatezza dell'umanità ingentilita, corrompersi, imbarbarirsi con la «barbarie della riflessione », e ricadere in una sorta di nuova ferinita, per ripassare dipoi a nuova eroica barbarie. 


\section{XVIII}

La barbarie ritornata o il Medioevo

$\mathrm{D}_{\mathrm{i}}$

tali ricorsi il Vico non studia e non addita se non un caso solo: il periodo di storia europea che, appunto ai suoi tempi, si era cominciato a piú particolarmente delimitare dagli storici e a denominare (benché il Vico non lo denomini ancora cosí) il "Medioevo".

Che quello fosse periodo di decadenza e di barbarie, non era di certo un pensiero che giungesse nuovo alla coscienza; perché, specie dal tempo dell'umanesimo, si era sentito il distacco e l'orrore verso quei secoli \& media et infimce latinitatis ", nei quali si trascurarono e dispersero i tesori delle letterature classiche, e i buoni studî languirono o si spcnsero affatto. E piú viva e piena questa coscienza, generale nell' Europa colta, era in Italia, la quale non poteva dimenticare che, se per altri popoli s'inizio in quel periodo la fortuna, la potenza e la civiltà, per lei il medioevo fu la fine dell'alma Roma, anzi l'avvilimento del nome romano e sorgente sopr'esso l'orgoglio vandalico, visigotico e langobardico, la devastazione delle ricche città, la distruzione dei maestosi monumenti, cle ancora mostravano dappertutto, nei loro ruderi, la lamentabile ruina. Il Machiavelli aveva dato principio alle sue storie con la concitata descrizione, che tutti conoscono, del cangiamento to- 
tale, seguito alla caduta dell'Impero d'oceidente. Ma passare a rassegna le rovine o raccogliere le antichità medievali non significava ancora penetrare nell'intimo spirito di quel tempo; cosí come notare le differenze e le deficienze d'un uomo rispetto a un altro non, vuol dire intendere lo spirito di quell'uomo e neppure quello dell'altro. Il Vico inaugura l'intelligenza dello spirito medievale, cioè della costituzione mentale, sociale e culturale di quell'età.

Egli, che pur viveva in una parte d'Italia dove molte erano, nonché i documenti, le persistenze del medioevo, confessa che quei tempi della barbarie seconda gli erano riusciti assai piú oscuri dei tempi della barbarie prima, dai quali anzi aveva tratto luce per comprendere l'altra. E l'aveva compresa nell'atto stesso che l'aveva cosi battezzata: «barbarie seconda 0 «ritornata 0 « ricorsa , considerandola come esempio della legge ideale che egli avera stabilita dei ricorsi. Il medioevo gli apparve, iu generale, come un ripresentarsi di condizioni primitive di vita e un conseguente riprodursi del processo sociale che da queste si svolge. Pensiero quanto originale altrettanto ricco di verita, al quale sarebbe fuori luogo opporre che il Vico scopre i caratteri generici e non i tratti individuali del medioevo; perché noi sappiamo che tale per l'appunto era il suo problema, la ricerca dei caratteri generici ossia delle uniformiti, e che egli schivava la storia propriamente detta per non essere condotto al bivio tra la scienza e la fede, tra la concezione affatto immanente della storia e perciò escludente rivelazione e miracolo, e quella affatto trascendente e, come miracolosa, ribelle alla trattazione scientifica. Anche ai giorni nostri (la cosa è degna di essere notata) abbiamo veduto risorgere codesto tentativo di rendere comportabili tra loro religione e storia, col prescindere dall'aspetto individuale degli avvenimenti 
e ridurre la storia a storia d'istituzioni e di uniformita ${ }^{1}$. In questa posizione, che il problema prende nel Vico, si trova anche la ragione del fatto, clıe è parso assai strano in un cattolico, del nessun risalto da lui dato al cristianesimo, nel quale egli s'ineontra proprio all'aprirsi del medioevo e di cui si spaccia in poche parole col dire: che Dio, avendo per vie sovrumane schiarita e ferma la verità della religione cristiana con la virtú dei martiri contro la potenza di Roma c con la dottrina dei padri e coi miracoli contro la vana sapienza greca, e dovendo sorgere nazioni armate da combattere in ogni parte la divinita del suo antore, permise che nascesse nuovo ordine di umanità tra le nazioni, perché la vera religione si fosse stabilita secondo il corso naturale delle cose umane.

Appaghiamoei dunque delle somiglianze che il Vico viene notando tra la societa medievale e quella dei primi secoli di Grecia e di Roma, e non prendiamo scandalo neppure se molto spesso le sue esemplificazioni e documentazioni riescano fallaci e immaginarie. Il suo pensiero storico sostanziale (come già sappiamo) è tanto gagliardo che passa attraverso gli errori, o a essi vive in mezzo senza esserne consunto. - Ecco (per rifare, riordinandolo, il suo raceonto o il suo quadro), ecco nel medioevo risorgere dappertutto gruppi di case sui monti, e sopra il caseggiato appollaiarsi le ròcche, come gia nell'età divina dei * ciclopi »; perché altro modo di scampo non v'era per le misere genti, battute dalla violenza dei barbari invasori e dalle lotte intestine. Le città piú antiche, sorte nei tempi di mezzo, e quasi tutte le capitali dei popoli, sono, infatti, poste sulle alture; gl'italiani chiamano « castella » tutte le nuove signorie che allora si formarono; e perciò anche,

1 Si vedano (a proposito di un libro del Sorel) le mie Conversazioni critiche, I, 310-22. 
forse, i nobili si dissero « summo o « illustri loco nati», laddove i plebei, che abitavano le valli e pianure sottostanti, « imo, obscuro loco nati ». Ecco riaprirsi gli asili, specialmente presso i signori ecclesiastici, che in tanta ferocia di vita erano piú mansueti; e gli oppressi e gli spauriti portarsi presso costoro e porre sé stessi e i loro patrimonî sotto la loro protezione. Perciò nella Germania, che dovette essere piú fiera che le altre parti d'Europa, restarono quasi piú sovrani ecclesiastici, vescovi e abati, che non secolari. Un illustre esempio di queste formazioni politiche era, nel regno di Napoli, la badia di San Lorenzo di Aversa, alla quale s'incorporò quella di San Lorenzo di Capua, e che nella Campania, nel Sannio, nella Puglia e nell'antica Calabria, dal fiume Volturno fino al mare piccolo di Taranto, governava centodieci chiese o direttamente o per abati e monaci a lei soggetti, e di quasi tutti quei luoghi gli abati di San Lorenzo erano signori o baroni. E poiché nei luoghi erti e riposti si edificavano piccole chiesicciuole per celebrarvi la messa e per compiere gli altri uffizî di pietả, quelle diventarono naturali asili dei popoli, che vi vennero fabbricando attorno i loro abituri: che è la ragione per cui dappertutto in Europa tante citta, terre e castella s'incontrano con nomi di santi e le chiese sono i monumenti piú antichi di quei tempi. Ed ecco, per conseguenza, in tutta l'Europa, non già formarsi per la prima volta ma tornare al mondo la feudalità, che si vaneggia uscita dalle scintille dell' incendio che i barbari dettero al diritto romano (come sostengono l'Oldendorpio e tanti altri giuristi), quando invece il diritto romano esso nacque dalle scintille dei feudi celebrati nella prima barbarie del Lazio, e la feudalita medievale non fu diritto nuovo delle genti di Europa, ma un diritto antichissimo, rinnovellato con l'ultima barbarie. Altro che " nateria vile» (come, al dire del Vico, la chiamava il Cuiacio) quella dei feudi, che è 
invece tutta eroica e degna di essere adornata della piú colta e riposta erudizione greca e romana! E perché mai se non per questa sostanzialc identità di natura le piú belle espressioni della giurisprudenza romana (con le quali lo stesso Cuiacio mitiga la barbarie della dottrina feudalc) riescono tanto acconce, che non si potrebbe desiderare di piú, ad esprimere le proprictà e gli attributi dei feudi? -Ritornò dunque nel medioevo la fondamentale divisione tra eroi e famoli, tra «viri» o «baroni 》 ( varones »si chiamavo ancora gli « uomini », $\mathbf{i}$ «viri », in lingua spagnuola) c semplici "homines ", come erano detti i vassalli, tra "patres ", o "patroni » o " padroni », e servi. I feudisti eluditi, che traducono "feudum " in "clientela", fanno ben piú di un'abile sostituzione linguistica, come s'immaginano, c, senza saperlo, definiscono storicamente il feudo. E i primi foudi del medioevo dovettero essere personali, come le prime clientele di Romolo: forma di vassallaggio che si osservava ancora ai tempi del Vico nei regni del settentrione, e specialmente in Polonia, dove gli "kmet» erano una specie di schiavi che quei «palatini » (o voievoda) spesso mettevano come poste dei loro giuochi, perlendone le intere famiglie che passavano cosí a nuovi padroni. Vennero poi i feudi rustici, di specie reale, consistenti in terre incolte che $i$ vincitori assegnavano ai popoli vinti per sostentamento, ritenendo essi le terre colte, e che $\mathbf{i}$ feudisti traducevano (con un'altra eleganza latina e con non minore verità storica) « beneficia». Gli antichi « nexi » furono i nuovi uomini « ligi » o \& legati », che dovevano riconoscere amici e nemici tutti gli amici e nemici del loro signore e che prestavano quella che in Roma si disse «opera militaris» e nel medioevo si ridisse « militare servitium ». Il legame feudale si estese a piú larghi rapporti politici; e come già i re vinti diventavano alleati e socî di Roma e « servabant maiestatem populi romani», 
cosi si ebbero feudi sovrani soggetti a maggiori sovranitì, i rappresentanti delle quali, grandi re e signori di grandi regni e numerose provincie, presero titolo di « maestà 》.

Le repubbliche risorsero aristocratiche, se non * di stato », per forma esterna di costituzione, per lo meno «di governo », quanto a effettivo potere politico: il che veniva confessato dai politici e fra gli altri dal Bodin, il quale del suo regno di Francia giunse a dire che, durante le due linee merovingia e earolingia, fu addirittura aristocratico di stato. E aristocratici si serbarono fino al secolo decimosesto, viventi testimonî del passato, i regni di Svezia e di Danimarca, e si serbava ancora, ai tempi del Vico, la Polonia, già ricordata. I primi pubblici parlamenti di Europa dovettero essere composti, come il senato di Romolo, dagli anziani della nobilta, dai « seniores 》 (donde «signori »); ed erano corti armate, come gli antichi comizî curiati, di baroni ossia di pari. In quei parlamenti si discettavano cause feudali intorno a diritti o successioni o devoluzioni di feudi per cagioni di fellonia o di caducazione; le quali cause, confermate piú volte con tali giudicature, fecero le consuetudini feudali. Un'ombra di quei parlamenti sembrava al Vico il Sacro Regio Consiglio di Napoli, al eui presidente si dava il titolo di « Sacra Real Maestà », come ai consiglieri il nome di « milites », e dalle sue sentenze non v'era appellazione ad altro giudice, ma solamente richiamo al medesimo tribunale.

Governi aristocratiei, e tutti avvolti da un nimbo o da una nube religiosa; tantoché non solo i vescovi e gli abati erano, come si è visto, assai spesso feudatarî, ma i feudatarî e i sovrani si coprivano d'insegne religiose, e i re cattolici dappertutto, per difendere la religione cristiana della quale sono protettori, vestirono le dalmatiche dei diaconi, consacrarono le loro persone reali (onde « sacra real maestà ») e presero dignità ecclesiastiche, come Ugo 
Capeto che s'intitolava conte e abate di Parigi; c duchi e abati, o conti e abati, s'intitolavano comunemente, come appare dalle antichissime scritture, i principi di Francia. Quei primi re cristiani fondarono religioni armate, con le quali difesero il cattolicesimo contro ariani, saraceni e altri infedeli. Ritornarono con verita $\mathrm{i}$ "purce et pia bella" dei popoli eroici: la croce sopra un orbe, che tutte le potenze cristiane sostengono sulle loro corone, ricorda le croci spiegate in campo nelle guerre sante, nelle crociate. Ritornarono le schiavitú eroiche, che durarono molto a lungo tra le nazioni cristiane, perché, considerandosi le gucrre giudizî di Dio, i vincitori credevano che $i$ vinti fossero $a b$ bandonati da Dio e li tenevano nientemeno che bestie (cosí i cristiani chiamano « cani » i turchi, e questi li ricamhiano col nome di "porci»). Gli antichi toglievano ai vinti "divina et humana omnia »; e i barbari nuovi, nell'occupare le citta, non ad altro attendevano che a ricercare e portar via i depositi o reliquie di santi (che $i$ popoli in quei tempi sotterravano e nascondevano con ogni diligenza), onde in quei tempi avvennero quasi tutte le traslazioni dei corpi santi. Di siffatti costumi è restato come vestigio che i popoli vinti debbano riscattare dai generali vittoriosi tutte le campane delle citta prese.

Analoghe somiglianze si ravvisano nell'ordinamento giuridico della proprieta. La somma divisione delle cose in diritto feudale è quella dei beni feudali e dei beni allodiali. Ma gli allodiali erano in origine di un diritto fortissimo, non infievolito da niun peso stranicro, neppure pubblico: erano $\mathbf{i}$ beni direttamente acquistati e conquistati dai patrizî o baroni, laddove i feudali importavano la laudazione del signore, dal quale erano stati concessi. Gli allodiali rispondevano dunque al dominio quiritario, "ex iure optimo ", c i feudali al bonitario; e solamente quando, in tempi piú tardi, nella nuova Europa, come gia 
in Roma antica, si formò un nuovo censo ed erario, e gli allodiali furono sottomessi ai pesi pubblici, poté nascere la denominazione dispregiativa di essi come «beni del fuso », in contrasto coi feudali, « beni della lancia ». Cosí, a mo' di esempio, le provincie che di poi s'incorporarono nel regno di Francia, erano state un tempo signorie sovrane, soggette feudalmente al re di quel regno, nelle quali i principi sovrani avevano i loro beni (allodiali), liberi di ogni pubblico peso; ma, poiché per successione, ribellione o decadenza vennero a far parte del regno, quei beni furono sottoposti ai dazî e tributi e il dominio \& ex iure optimo s si confuse col dominio privato non feudale, soggetto a quei pesi, e gli allodî nel significato nobile divennero una cosa sola con gli allodî nel significato volgare. I feudisti degli ultimi tempi smarrirono il valore della distinzione originaria, come i giuristi romani ultimi non averano inteso il significato del dominio « ex iure optimo *. Al dominio feudale si collegavano le enfiteusi, onde il laudemio restò a significare egualmente ciò che il vassallo paga al signore e l'enfiteuticario al padrone diretto; le " commende ", che erano le antiche clientele; $\mathrm{i}$ « censi , per effetto dei quali si era tenuti a servire nelle guerre $i$ nobili (e i censuanti, o gli * angarî * e « perangarî ", ríprodussero gli «assidui» romani); le «precarie », che dovettero essere dapprima le terre date dai signori alle preghiere dei poveri; $i$ * livelli * o permutazioni di beni stabili, che in quell'economia agricola tenerano luogo dei commerci mancanti. L'esclusione delle donne dalle successioni, ch'era dell'antichissimo diritto romano, si celebrò di nuovo nella " legge salica ", in origine (congettura il Vico) comune alla Germania e a tutte le altre prime barbare nazioni di Europa, e poi restata in rigore solamente nelli Francia e nella Savoia.

I castighi erano crudeli e la pena di morte si disse 
«pena ordinaria ». Ma neppure nel medioevo c'erano vere leggi e procedimenti penali pei torti privati: gli ammazzamenti dei plebei o accadevano per fatto dei loro padroni medesimi, ehe niuno poteva accusare; o per fatto d'altri, e come di servi altrui si rifaceva al padrone il danno, secondo ancora si costumava nella Polonia, nella Lituania, nella Danimarca, nella Svezia e nella Norvegia. Col nome di «purgazioni canoniche» (non riconosciute per altro dai sacri canoni) si celebravano in tutta Europa certe specie di giudizî divini o duelli; e, fino ai tempi di Bartolo, vi ebbero vigore le rappresaglie. Nei giudizî intorno agli allodî i signori se la vedevano tra loro con le armi; e nel reame di Napoli, ancora ai tempi del Vico, i baroni non coi giudizî civili ma con duelli vendicavano gli attentati fatti dentro i loro feudi da altri baroni. In una societa dove la forza imperava, qual maraviglia che tornassero altresi i ladronecci eroici, e che il titolo di «corsaro " o * pirata divenisse titolo di signoria? Né mai, come allora, fu tanto varia e incostante la fortuna dei regni.

Il diritto romano giustinianeo, tutto penetrato dall'idea di equità, fu rifintato e cadde in oblio. In Francia e in Ispagna era gravemente punito chi osasse allegarlo nelle cause; e certamente in Italia si recavano a vergogna $i$ nobili di regolare i loro affari con le leggi romane e professavano di vivere secondo quella langobardica, laddove i plebei, cle piú tardi si disavvezzano dagli antichi costumi, praticavano alcuni diritti romani in forza di consuetudini. $\mathrm{E}$ in consuetudini piuttosto che in leggi consistevano i diritti allora vigenti, nei quali ripresero importanza le formole rigide e le cerimonie solenni e si distinsero i patti cosí detti « nudi » da quelli « vestiti », cioè sussidiati da tali formole e cerimonie. Della reverenza per le formole può valere come esempio la leggenda (al tempo del Vico creduta fatto storico) di quel che intervenne all'imperatore 
Corrado III allorché prese Weinsberga, città che avevo fomentato il suo competitore nell'impero e ehe egli condannò allo sterminio, dando legge che fossero salve le sole donne con quanto potessero trasportare addosso. Ma quelle donne uscirono dalla condannata citta, eariche dei loro figliuoli, mariti e padri; e l'imperatore, che stava alla porta, a capo dell'esereito, pronto con le spade sguainate e con le lance in resta a vendicare la collera sua spaventosa, vide e sofferse che passassero salvi tutti, per rispetto alla formola letterale della legge da hi decretata.

Erano tempi analfabetici, il che il Vico esprine con la sua immagine che tornarono allora i linguaggi * mutoli * o « geroglifiei ». Le lingue volgari, italiana, francese, spagnuola e tedesca, non erano messe in iseritto, e solo si scriveva da pochi ecclesiastici un barbarico latino, onde " chierico» significò « uomo dotto »; ma tra gli stessi sacerdoti regnava tanta ignoranza che si vedono scritture firmate da vescovi col segno della eroce, perehé non sapevano scrivere neppure i proprì nomi. Per sí fatta scarsezza una legge inglese disponeva che un reo di morte, che sapesse di lettere, come eccellente in arte non fosse messo a morte; e "letterato ", come " ehierico», si stese a significare l'erudito. Di qui l'importanza e il largo uso ehe ebbero le imprese gentilizie per accertare $\mathrm{i}$ dominî sopra ease, sepolcri, campi e armenti, delle quali tante si osservano ancola sugli edifizî di quei tempi.

Con la barbarie tornò altresí il predominio del verso sulla prosa. Le prose dei padri della chiesa latina (e il medesimo si dica di quelli della greca) sono piene di numeri poetici, talché sembrano cantilene. La prima lirica nuova fu religiosa; e se non si ebbero propriamente poemi religiosi eristiani, fu perché le cose della nostra teologia superano ogni senso e ogni immaginazione e spossano di troppo la facoltì poetica. Poesia e storia si riconfusero; $\mathrm{i}$ 
romanzieri, che furono i poeti eroici dei tempi barbari ritornati, credettero di narrare storie vere; onde poi il Boiardo e l'Ariosto presero i soggetti dei loro poemi dalla storia di Turpino, vescovo di Parigi. E quando, per effetto della famosa scuola parigina e della sottilissima teologia scolastica, la lingua francese passò di colpo dalla spontaneità alla riflessione, come essa lingua scrbò in gran numero i dittonghi pur riempiendosi di termini astratti, cosí la storia di Turpino sopravisse in Francia quasi poema omerico. Gli stessi facitori dei poemi in latino non cantarono altro che storie, come Gugliclmo pugliese nel De gestis normannorum in Italia e Guntero nel Carmen heroicum de rebus a F'rederico Barbarossa gestis. $\mathrm{E}$ poeti furono i primi scrittori del volgare in Italia non meno che in Provenza e in Francia. Risorse la virtú puntigliosa, eome quella di Achille, nella quale tutta la loro morale riponevano i duellisti; donde uscirono le leggi superbe, gli uffizî altieri e le soddisfizioni vendicative dei cavalieri crranti, eantate dai romanzieri. Non sembra proprio un personaggio omerico dalla rapida commozione quel Cola di Rienzo, che (come si legge uella vita di lui), mentre mentova l'infelice stato di Roma, esso e coloro coi quali ragiona, prorompono tutti in dirottissimi pianti? L'iperbole fantastica era ancora una condizione comune di spirito, cone nei fanciulli; perché (scrive il Vieo) \& sovente io mi ricoldo, quando vado a pásseggio, che i molli clivi che mi si svolgono innanzi agli occhi, parevano a me bambino altissime montagne e dirupate ». Cosí, nel medioevo, gli Orlandi e gli altri paladini erano finti di enorme statura; e le immagini degli esseri divini, del Padre eterno, di Cristo, della Vergine, si dipingevano e scolpivano di eccedente grandezza.

Ma gl'ingegni umani sono a guisa dei terreni i quali per lunghi secoli incolti, se una volta si riducono a col- 
tula, dànno nel bel principio frutti maravigliosi in perfezione, grandezza e abbondanza. Epperò al tempo della spirante barbarie d'Italia, dopo quattro secoli dei piú crudeli e tempestosi, nacque Dante, l'Omero della ritornata barbarie; come, poco stante, fiorí la poesia delicata del. Petrarca e la prosa leggiadra e graziosa del Boccaccio: esempî tutti e tre incomparabili. E per la natura già definita della barbarie, naturalmente veritiera, aperta, fida, generosa e magnanima, Dante espose in comparsa persone reali e rappresentò fatti reali dei trapassati; e il suo poema chiamò " commedia * in ricordo dell'antica commedia greca, che teneva il medesimo procedere. Nel qual poema sembrano avere corrispondenza cosí l'Iliade come l'Odissea: la prima, nell'Inferno, dove Dante impiegò il suo collerico ingegno e tutto il grande della sua fantasia nel narrare tante jre implacabili e nel rimembrare un gran numero di spietatissimi tormenti, degno riscontro alle tante forme atroci di morte descritte da Omero (le quali descrizioni, che ora destano pietả, agli uditori contemporanei cagionavano, invece, piacere); - la seconda, cioè l'Odissea, che celebra l'eroica pazienza di Ulisse, trova rispondenza nel Purgatorio, spettacolo di pene tormentose sofferte con inalterabile pazienza, e nel Paradiso, dove si gode infinita gioia con una somma pace dell'animo. E un'altra somiglianza di Dante con Omero è nella fisionomia della lingua da lui adoperata, cosí varia che si è opinato che egli l'avesse raccolta, come Omero quella dei suoi poemi, da tutti i dialetti della sua nazione: opinione dei dotti del secolo decimosesto, la quale non regge alta critica, perché è indubitabile che, quando Dante li usò, quei parlari dovevano essere comuni in Firenze, né vita d'nomo gli sarebbe bastata ad andiarli raccogliendo in tempi in cui dei varî dialetti mancavano gli scrittori. Ma la piú sostanziale somiglianza che egli ha con Omero è nella sublimita poetica. 
Dante è un divino poeta che alle fantasie delicate moderne sembra incolto e ruvido, e agli orecchi ammorbiditi da musiche effemminate suona un'ar'monia soventi volte insoave; ma non cosí agli uomini di gusto austero, che non badano a dilettarsi di fiori, acconcezze e amenità. E, come Omero, egli è grande non per la sua sapienza riposta, ma per la sua vigorosa fantasia. Certo, Dante fu dottissimo in divinita; ma in ciò, piuttosto che la sua forza, è la sua debolezza. Se Dante non avesse saputo né di scolastica né di latino, sarebbe riuscito anche maggior poeta, e forse la. lingua toscana avrebbe avuto (come non ebbe la latina) un poeta da contrapporre in tutto e per tutto a Omero. Il modo acconcio di commentarlo è di dare notizie delle cose, fatti o persone mentovati dal poeta, di spiegare $i$ sentimenti di lui, e tralasciare "ogni morale e molto piú scienziata allegoria ».

Colui che scrivera questa pagina di critica dantesca, e rivendicava Dante dopo secoli di gusto antidantesco (o dantesco-grammaticale e dantesco-scolastico), e lo rivendicava proprio negli anni della fioritura arcadica cosi ritrosa e avversa a Dante, avrebbe meritato d'incontrarsi col genio di Guglielmo Shakespeare, che forse egli solo, a quei tempi, era in grado d'intendere nella sua possanza poetica. Ma in Italia, come in generale fuori d'Inghilterra, dello Shakespeare allora non si conosceva nulla; e appena ne giungeva al Vico una tarda e vaga notizia, cioè che gl'inglesi, non ammolliti dalla delicatezza del secolo, non si dilettavano di tragedie che non avessero dell'atroce, appunto come il primo gusto del teatro greco fu delle nefarie cene di Tieste e dell'empie stragi compiute da Medea su fratelli e figliuoli. I a tendenza verso la poesia e la letteratura germanica rimase nel Vico, come sappiamo, un sospiro: non gli era possibile scorgerne i precisi e particolari lineamenti per quanto appuntasse cola lo sguardo, e 
una volta che vi accennò, sopra notizie di seconda mano, fu per affermare che, nella nazione tedesca, particolarmente nella Slesia provincia tutta di contadini, «nascono naturalmente poeti »; cioè, andando in cerca di poesia ingenua e popolare, gli accadde di abbracciare, senz'avvedersene, i rappresentanti della scuola slesiana, gli Hoffmannswaldau e i Lohenstein, imitatori tedeschi del suo compaesano cavalier Marino! Ma questo è un aneddoto, atto non ad altro che a confermare con nuovo esempio gli scherzi che faceva al Vico la sua accesa immaginazione.

Come si uscisse dalla seconda barbarie e dalla costituzione feudale, il Vico non dice; e sembra non aver fermato la sua attenzione sul movimento comunale, che tante analogie gli avrebbe offerto con lc lotte della plebe roinana e col formarsi dell'antica democrazia. Schernisce, per altro, anche qui, coloro che facevano sorgere le monarchie moderne, per es., la francese, di punto in bianco, con una sorta di legge di Triboniano, per la quale (dice ironicamente) i paladini di Francia si sarebbero spogliati della loro potenza, conferendola nei re della linea capctingia. Nota anche che, essendosi sparso e quindi disperso il potere dei baroni per effetto delle guerre civili nelle quali dovevano dipendere dai popoli, fu facile raccoglicrla nei re monarchi, mutandosi cosí l'ossequio dei vassalli al barone nell' "obsequium principis ". Ma speciale importanza egli dà al risorgere della giurisprudenza romana (di questo "diritto naturale delle genti di Europa », come lo aveva chiamato il Grozio) con gli studi aperti nelle università d'Italia; onde si appresero di nuovo i principî dell'equita naturale, i nobili e gl'ignobili furono adeguati nella ragion civile come gia sono nella natura umana, l'arcano delle leggi uscí di mano ai feudatarî, dei quali per tal modo venne sempre piú fiaccata la potenza; e si giunse al governo umano delle repubbliche libere e delle 
perfettissime monarchie. La già ricorsa società eroica aveva sofferto ormai il contrario ricorso; e, nella civiltà dei tempi nuovi, era impossibile richiamarla in vita, come vani riuscirono i tentativi di restaurazione aristocratica che fecero nell'antichità i pitagorici e Dione siracusano. Gli uomini plebei, una volta che si sono riconosciuti di egual natura coi nobili, non sopportano piú di restare inferiori a essi nella vita civile. E le pochissime repubbliche aristocratiche, che qua e là avanzavano in Europa, dovevano adoperare molte sollecite cure e accorti e saggi provvedimenti per tenere a freno e contente le moltitudini da esse governate. 


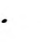




\section{XIX}

IL VICO CONTRO L'INDIRIZZO DI CULTURA DEI SUOI TEMPI

G

Tiunto, nel contemplare il corso della storia, ai tempi, che erano i suoi, di umanità sparsa su tutte le nazioni, il Vico fa una rapida deserizione del mondo contemporaneo, e tace, se non soddisfatto, incerto o prudente. Come non era salito alla Scienza nuova per diretto impulso di problemi politici (almeno nel significato ehe hanno d'ordinario queste parole), cosi dalle contemplazioni della Scienza nuova non discese alla vita attiva, neppure nella forma che si presenta piú ovvia a un uomo di pensiero, del libro e dell'opuscolo che critichi leggi e istituti o proponga riforme. Anche quando, per poco, concepí una « pratica della sua Scienza', non pensò che questa pratica, per la parte ehe gli spettava, potesse aggirarsi altrove che « dentro le accademie $\gg$.

La pratica « dentro le accademie», ossia nel campo della eultura, è, per altro, pratica anch'essa e politica; e non di certo tra le meno importanti. E uno storico o un filosofo non può astenersene mai del tutto, se anche l'aceentui piú o meno fortemente, la svolga con maggiore o minore ampiezza.

1 Si veda sopra, cap. IX, pp. 110-13. 
Il Vico v'insisté e la svolse ampiamente; anzi, la sua prima affermazione nella vita scientifica fu appunto l'esame dei metodi moderni di studio e di educazione, comparati con quelli degli antichi : esame il quale (dopo saggi e tentennamenti nelle prime orazioni) prese corpo nella prolusione universitaria del 1708, De nostri temporis studiorum ratione. Negli anni che seguirono, tutt'intento a lavorare alla Scienza nuova, non dette altre pubbliche manifestazioni del suo malcontento verso l'indirizzo che prevaleva negli studî; ma con tanto maggiore frequenza e vivacita si espresse sul proposito nelle lettere private e nelle conversazioni, né volle tralasciar di ricordare quel ch'egli ne pensasse nell'autobiografia. Non fa d'uopo dunque ricavare il suo atteggiamento polemico dai sottintesi e dagli incisi della sua opera capitale, perché egli medesino in piú occasioni rese espliciti i sottintesi e mutò gl'incisi in proposizioni principali.

Quella polemica si mosse in due campi attigui, che rispondono al duplice aspetto della Scienza nuova, come filosofia dello spirito e come scienza generalizzante. Sotto il primo aspetto, il Vico aveva fatto valere i diritti della fantasia, dell'universale fantastico, del probabile, del certo, dell'esperienza, dell'autorità, e quindi della poesia, della religione, della storia, dell'osservazione naturalistica, dell'erudizione, della tradizione. Sotto il secondo, aveva disegnato uno schema dello svolgimento naturale dello spirito, cosi nella storia del generc umano come nella vita individuale, messa da lui a continuo riscontro con le fasi della storia. Perciò il suo esame doveva volgersi di neeessita, per una parte, alle disposizioni spirituali del suo tempo e, per l'altra, al modo in cui s'intendeva e s'impartiva allora l'educazione dei fanciulli e giovinetti. Nell'uno e nell'altro campo, il Vico avvertiva le medesime deficienze, ed era offeso da quel medesimo arido intellettua- 
lismo che aveva reso inintelligibile il processo della mente, e mutilato e falsato la verita della storia umana.

Ai fanciulli, appena usciti dalle scuole di grammatica, si soleva infliggere un'istruzione di logica, adoperando, secondo le predilezioni dei maestri, ora la logica scolastica, ora c piú di frequente quella (in sostanza anch'essa aristotelica e scolastica), che era stata elaborata dall'Arnaud e si diceva " logica di Portoreale ", tutta ripiena di severissimi giudizi intorno a materie riposte di scienze superiori e lontane dal comune senso volgare, tutta infarcita di esempî tratti da tali scienze. I fanciulli avrebbero dovuto, mercé quella disciplina, farsi d'un tratto critici e purgare Ia mente non solo del falso, ma perfino delle opinioni verisimili e probabili; quando, in verita, di niente si potevano purgare, perché la loro mente era ancora sgombra o scarsamente fornita, e nessuna critica potevano esercitare, per mancanza di materia criticabile : avrebbero dovuto essere condotti a ben giudicare innanzi di ben apprendere, contro il corso naturale delle idee, che prima si apprende, poi si giudica e finalmente si ragiona. Il risultato era che gli spiriti a quel modo educati diventavano aridi e secchi nello spiegarsi e, senza far mai nulla, pretendevano giudicare d'ogni cosa; restavano tutta la vita afflatissimi nella maniera di pensare e inabili a ogni grande lavoro: critici, per cosi dire, ma sterili. Donde non solo leggerezza e arroganza nei giudizî, ma incapacita nella pratica della vita, nel trattare che si usa con gli uomini, nella eloquenza civile, la quale si fonda non tanto sulla critica quanto sul verisimile e deve ottenere il suo effetto, col dire cose opportune, penetrando la psicologia dell'interlocutore e'regolandosi in modo conforme. Della prematura educazione logico-critica il Vico aveva fatto esso medesimo dannosa esperienza; quando, a lui fanciullo, il gesuita Del Balzo, che fu tra $i$ suoi primi maestri, mise in mano 
l'opera del sommolista Paolo veneto; e l'ingegno, ancora debole da reggere a quella specie di logica crisippea, poco mancò non vi si perdesse, tanto che, per disperazione fatto disertore degli studî, ne divagò un anno e mezzo. Migliore ricordo serbava, invece, perfino delle capestrerie poetiche giovanili, alla scuola dei piú sfrenati marinisti napoletani: divertimento (egli dice) poco meno che necessario per gl'ingegni dei giovani, assottigliati di troppo e irrigiditi nello studio delle metafisiche in quegli anni per l'appunto in cui l'ingegno, per l'infocato vigore dell'età, deve dare in trascorsi perché non si assideri e dissecchi affatto. Quell'etì, che è la barbarie dell'intelletto, cosí vigorosa di fantasia e (per gli stretti rapporti che sono. tra queste due facolta) di memoria, richiede di essere nutrita ed esercitata con la lettura dei poeti, storici c oratori, e con l'apprendimento delle lingue. L'arte che vi si deve insegnare non è la critica ma la topica, la vera arte dell'ingegno, ossia della facoltà d'inventare; per la quale i fanciulli vengono apparecchiando la materia per giudicare bene dipoi, perché non si giudica bene se non si è conosciuto il tutto della cosa e la topica fa ritrovare per ciascuna cosa tutto ciò che vi è dentro. Per tal modo, i giovinetti, seguendo il corso stesso della natura, si formano e filosofi e ben parlanti.

Un correttivo, certamente, è indispensabile all'esuberante fantasia; ma non deve cercarsi nella logica, sí piuttosto nella geometria lineare, che è in qualche modo una pittura, e invigorisce la memoria col gran numero dei suoi elementi, ingentilisce la fantasia con le sue delicate figure e fa spedito l'ingegno nel doverle correre tutte e fra tutte raccogliere quelle cle fanno al caso per dimostrare la grandezza domandata. Senonché, anche per la geometria ogni frutto veniva guasto dal metodo favorito nelle scuole di allora, che era l'algebraico; il quale, non meno della 
logiea seolastica, assidera tutto il piú rigoglioso delle indoli giovanili, loro accieca la fantasia, spossa la memoria, infingardisce l'ingegno, rallenta l'intendimento, dannegyiando per queste quattro vie le arti belle, la conoscenza delle lingue e delle storie, Ie invenzioni e la prudenza. Piú particolarmente, l'algobra affligge l'ingegno, perché esso con quel. metodo non vede se non eiò soltanto che gli sta innanzi ai piedi; sbalordisce la memoria, perché, ritrovato il secondo segno, non bada piú al primo; abbacina la fantasia, perehé non immagina affatto nulla; distrugge l'intendimento, perché professa' d' indovinare. I giovani che vi hanno speso molto tempo, messi poi a trattare gli affari della vita civile, con loro sommo rammarico e pentimento vi si ritrovano meno atti. Onde, perehé recasse alcuna utilita e non facesse niuno di sí gravi danni, si dovrebbe apprendere per poco tempo alla fine del corso matematico e servirsene solo come mezzo abbreviativo. L'abito del ragionare si forma assai meglio con l'analitica metafisica, che in ogni questione prende il vero nell'infinito dell'ente, e pei generi della sostanza viene gradatamente rimovendo ciò che la cosa non è per tutte le specie, finché giunge all'ultima differenza costituente l'essenza della cosa clıe si desidera sapere.

Tutta l'educazione soffriva dell'eccesso di matematica e del difetto di concretezza; e come se i giovani dovessero dalle accademie uscire in un mondo umano, composto di linee, numeri e specie algebraiche, si riempiva loro il eapo dei magnifici voeaboli di « dimostrazioni », di « verita dimostrate, di « evidenze, e si condannava la regola del verisimile, della quale non hanno altra piú sicura i politiei nei loro consigli, i capitani nelle loro imprese, gli cratori nel trattare le cause, i giudici nel giudicarle, i mecliei nel eurare i unalati del corpo, i morali teologi nel curare quelli delle coscienze, e sopra la quale tutto il mondo 
si acqueta e riposa in tutte le liti e controversie; in tutti i provvedimenti, in tutte le elezioni, che tutte si determinano con l'unanimità o maggioranza dei voti. Si preparava una generazione vuota e prosuntuosa, saccente e non sapiente, razionatrice e povera di verità.

Quale l'educazione che somministravano, tali erano gli stessi educatori, ossia l'ambiente generale di cultura. La poesia era morta: quei metodi analitici assideravano * (giova adoperare ancora una volta la parola che il Vico ripete con frequenza come bene espressiva) \& tutto il generoso della miglior poesia' $\gg$. veramente non mai come in quella prima metà del settecento l'Europa fu cosí vasto deserto poetico: l'Italia si era ridotta al melodramma metastasiano; la Francia non aveva dato successori ai Corneille e ai Racine; in Ispagna, esaurita l'ultima grande manifestazione dello spirito nazionale, che fu il dramma, cominciavano l'imitazione francese e il razionalismo; l'Inghilterra sembrava dimentica affatto di aver dato i natali allo Shakespeare, e la Germania si trascinava anch'essa dietro l'imitazione neoclassica. E non solo non nascera nuova poesia, ma non se ne desiderava. Seguendo Cartesio e il Malebranche, i filosofi professavano di ammortire tutte le facoltà dell'anima che provengono dal senso, e specialmente la facoltà d'immaginare, che detestavano come madre di tutti gli errori. I poeti erano da essi condannati col falso pretesto che narrassero "favole »: come se quelle favole non fossero le eterne proprietà degli animi umani, che $i$ filosofi politici, economici e morali ragionano e i poeti presentano in vivi ritratti.

Sull'autorita degli stessi cartesiani, lo studio delle lingue formava oggetto di disprezzo: non aveva detto Renato che sapere di latino non è sapere piú di quello che sapeva la fante di Cicerone? La severa conoscenza del latino e del greco era finita con gli scrittori del cinque e seicento; 
il culto delle lingue orientali si era ristretto nei protestanti; lavori sulla giurisprudenza romana si facevano ancora solo in Olanda. La famosa biblioteca napoletana del Valletta, ricca delle piú belle edizioni latine e greche, fu generosamente comprata dai padri dell'Oratorio, ma per meno della meta del suo primitivo valore, tanto era scaduta la richiesta di quei libri. In Francia la biblioteca del cardinal Dubois non trovò un unico eompratore e andò dispersa nella vendita spicciola. I prineipi non amavano piú il bel latino d'olo; e a nessuno di essi cadde in pensiero di far conservare all'eternita da penna eccellente latina un avvenimento cosi strepitoso come la guerra di sueeessione di Spagna, paragonabile per la sua complessità solo alla seconda cartaginese.

Assai si vantavano i metodi nuovi, ma niuno avrebbe potuto indicare le cose nuove, che si erano ritrovate per loro mezzo. Formole nuove e cose veechie; e, in compenso, vane lusinghe di poter giungere in brevissimo tempo e eon pochissima fatica a saper tutto. La dottrina civile e politica era trascurata per la fisica, la fisiea per la matematica; l'esperienza, poco esereitata; lo spirito inventivo del secolo precedente, quasi affatto spento. Lo scettieismo, conseguenza del metodo cartesiano, invadeva il campo del sapere.

Continuava altresi in quel tempo, per tutta Europa, il predominio della lingua francese: lingua la quale, diversamente dall'italiana, è ribelle alla poesia e all'eloquenza; ricea (dicera il Vico) nei vocaboli di sostanza, e, poiché la sostanza è eosa brutale e immobile e non patisce comparazioni, incapace di dare eolore alle sentenze, di amplificare e d'ingrandire, restia alle inversioni, sterile di metafore. I franeesi, piuttosto ehe periodi, posseggono membri di periodi; né la loro metriea ha versi superiori ai eosi detti alessandrini, che sono distrofi e più inerti e 
tenui degli elegiaci; né le loro parole patiscono altri accenti che non siano quelli delle due ultime sillabe. Impotente al sublime, la lingua francese è, per altro, attissima al piccolo, e per la già notata abbondanza di vocaboli di sostanza, ossia di termini astratti, al genere didascalico: in luogo dell'eloquenza, offre l'esprit. Degnamente, la nuora critica e l'analisi erano nate in Francia e si rivestivano di quella lingua.

La sola ricchezza, che in tanta povertì ogni giorno si accrescera, crano i ristretti, le biblioteche, i dizionarî delle scienze, come quelli che portavano i nomi del Bayle, dello Hoffmann, del Moreri: maniera d'apprendere la piú scioperata e casuale che si potesse mai inventare. Il genio del secolo era piú vago di esporre in compendio quanto altri seppe che di profondarsi per passare piú innanzi. Sembra cosa impossibile; eppure si compilarono, perfino, dizionarî di matematiche. Si provava da tutti un gran desiderio di scienza a vil mercato; e si stimavano buoni i soli libri spiegati e facili, che si prestavano a formar oggetto di ragionamento per passatempo con le dame, condannando, col dirli inintelligibili, tutti quegli altri che richiedevano nel lettore varia e copiosa erudizione e lo costringevano al tormento del molto riflettere e combinare.

Quei dizionarî e ristretti suscitavano nel Vico il ricordo di altri sinili lavori della decadenza greca, le ecloghe, i lessici e le biblioteche dei Suidi, degli Stobei e dei Fozî. E tutta la cultura contemporanea gli sembrava che rinnovasse il caso della sapienza greca e andasse a spirare in metafisiche inutili o dannose alla civilta, e in matematiche occupate in considerare grandezze che non sopportano riga e compasso e non hanno nessuna applicazione. Come altri savî uomini del suo paese, egli viveva persuaso che se la providenza divina, per una delle sue infinite secrete vie, non la invigorisse e rinfrancasse, la repubblica delle 
lettere correva rapidamente verso la fine. Dove era piú, allora, il sapiente, il vero sapiente, che il Vico aveva incontrato nella storia, dapprima nella figura barbarica del poeta teologo e poi in quella civile e razionale del filosofo greco e del giurisprudente romano, e che vagheggiava modernamente nel maestro di eloquenza (e si direbbe nella sua propria persona), come chiamato a dare unità, vita ed efficacia a tutto il sapere? La sapienza, infatti, non è questa o quella conoscenza o la somma delle conoscenze; ma è la facoltà che comanda a tutte le discipline e dalla quale si apprendono tutte le scienze e tutte le arti che compongono l'umanità. $E$ poiché l'uomo è mente e animo, intelletto e volontà, essa deve compiere all'nomo entrambe queste parti e la seconda come conseguenza della prima: deve insegnare la cognizione delle divine cose per condurre a sommo bene le cose umane. Il sapiente è l' nomo nella sua totalità e centralita, l' nomo intero.

Altissimo ideale, senza dubbio, come perfettamente giuste tutte le critiche mosse al metodo di educazione e alle tendenze spirituali del tempo suo. Eppure, fra tante e mirabili verita che si lasciavano addietro di lungo tratto il secolo decimottavo, si sente nel Vico, pedagogista e critico, qualcosia di retrivo. Si sente che egli, esclusivamente sollecito delle sorti della grande e severa scienza e fisso l'occhio nella forma piú compiuta dell'umanità, non intendeva il valore rivoluzionario di quello scetticismo e razionalismo e di quella ribellione al passato, che erano necessarî strumenti di guerra contro re, nobili e preti; di quei ristretti e dizionarî, che dovevano mettere capo all'Enciclopedia; di quella scienza popolare, che preludeva al giornalismo; di quei libercoli per dame e per eleganti conversazioni, che avrebbero alimentato i salotti del secolo decimottavo e temprati gli spiriti al radicalismo giacobino. Si sente in lui, qui come nel suo sistema filosofico, il cat- 
tolico che è avvinghiato al filosofo, il pessimista cristiano che grava sul dialettico dell'immanenza. Egli non sa scorgere il progresso nei suoi avversarî, e perciò non li riconosce quali veramente erano, a lui inferiori ma gradini da salire per giungere a lui, e che egli stesso avrebbe dovuto salire per intendere e posseder meglio sé stesso. Il suo atteggiamento polemico verso la cultura del tempo compie e conferma l'analisi, che si è data di sopra, delle virtú e insieme dei difetti della sua filosofia. 


\section{$\mathrm{XX}$}

\section{CONCLUSIONE}

\section{Il Vico E lo Svolginento posteriore DEL PENSIERO FILOSOFICO E STORICO}

Si aspetterebbe invano che noi, qui al termine della nostra esposizione, aggiungessimo un giudizio, e, come si suol dire, una «valutazione» dell'opera del Vico. Se il giudizio non si è già ottenuto come risultante dall'esposizione stessa, coincidente con essa; se storia e critica non banno fatto tutt'uno; la colpa sara stata o nostra o del lettore poco attento e, a ogni modo, in questo punto non rimeriabile con aggiunte che sarebbero appiccicature e con ripetizioni che sarebbero ridondanze.

Confessiamo anche di non nutrire simpatia per quei capitoli, che si usa mettere come conclusione dei lavori critici intorno ai filosofi e che contengono la storia della fortuna delle loro idee. Giacché se le « idee » sono prese in modo estrinseco, nei loro effetti suciali e di cultura, quella rassegna, sebbene non priva di certa sua particolare importanza ${ }^{1}$, rimane estranea alla storia della filosofia propriamente detta; - se invece sono intese come

1 Si veda Appendice, II. 
vere e vive idee filosofiche, il racconto della loro fortuna confluisce né piú né meno che con la storia della filosofia posteriore, la quale non c'è ragione di far seguire alla trattazione di uno piuttosto che di altro filosofo singolo. Condursi diversamente è effetto del pregiudizio che le idee siano qualcosa di solido o di cristallizzato, quasi gemme preziose che si tramandino da una generazione all'altra $\mathrm{e}$ di cui sia sempre possibile riconoscere l'inalterata forma e fulgore nei nuovi diademi che esse compongono c sulle nuove fronti che rengono a costellare. Ma le idee, effettivamente, non sono altro che l'infaticabile pensiero umano, e tramandarle davvero vale trasformarle.

Pure, è un fatto che nessuno ha scritto sul Vico che non abbia sentito il bisogno di spingere lo sguardo ai tempi posteriori e notare somiglianze e analogie delle dottrine di lui con quelle che seguirono cinquant'anni o un secolo dopo. E, quel ch'è piú, anche noi, nonostante la confessata antipatia e i professati criterî metodici, proviamo ora il medesimo bisogno. Perché? Perché il Vico ai suoi tempi passò per uno stravagante e rimase un solitario; perché lo svolgimento successivo del pensiero avvenne quasi tutto fuori della sui diretta efficacia; perché anche oggi, noto sufficientemente in alcuni circoli ristretti, non ha avuto ancora il posto che merita nella storia generale del pensiero. Ora, quale mezzo si offre piú semplice per mostrare la rispondenza delle sue dottrine, vere o false che fossero, a profonde esigenze spirituali, che ricordare le idee e i tentativi simili, riapparsi piú tardi cosí copiosi e intensi da dare la fisonomia al Iaroro di un secolo intero di filosofia e di storia? E se anche, dopo che noi abbiamo esaminato nell' intrinseco il pensiero di Iui, questo richiamo ai fatti che seguirono possa sembrare un espediente superfluo, si concederà per lo meno che, posto che la nostra come ogni altra orazione debba avere il suo compimento 
rettorico, nessuna perorazione si presenta piú spontanea di quella che consista in un rapido cenno della filosofia e della filologia posteriori, lumeggiate nelle loro attinenze col pensiero vichiano.

Si potrebbe anzi, col metodo che egli tenne per la barbarie seconda in confronto con la prima, presentare la storia ulteriore del pensiero come un ricorso delle idee del Vico. Ricorsero, in primo luogo, la critica di lui al sapere immediato di Cartesio e il suo criterio della conversione del vero col fatto, nel moto speculativo che andò dal Kant allo Hegel e che culminò nella tesi dell'identità del vero col fatto, del pensiero con l'essere. Ricorse la sua unita di filosofia e filologia nella rivendicazione della storia contro lo scetticismo e l' intellettualismo del secolo decimottavo, figlio del cartesianismo; nella sintesi a priori kantiana, che riconcilio ideale e reale, categoria ed esperienza; e nella filosofia storica dello Hegel, in cui lo storicismo del secolo decimonono tocè̀ il piú alto punto. Quell'unione di filosofia e filologia, che fu talvolta in lui unione violenta e commistione di metodi, ricorse anche in questa sua forma errata nella scuola hegeliana; talché siffatto indirizzo mentale potrebbe prendere a giusto titolo il nome di * vichismo ». Ricorse la limitazione che egli aveva tentata del valore delle matematiche e delle scienze esatte, e la sua critica della concezione matematica e naturalistica della filosofia, nella critica del Jacobi al determinismo spinoziano e in quella dello Hegel contro I'intelletto astratto; e, per le matematiche in particolare, fu (dal Dugald Stewart e da altri) messo in chiaro che la loro forza non è nei postulati ma nelle definizioni, e le * fictiones ", di cui egli parlava, sono rientrate nella terminologia odierna dei gnoseologi di quelle discipline. La logica poetica o la scienza della fantasia divenne l'Estetica, cosí ansiosamente indagata dai filosofi, letterati e artisti tedeschi nel corso 
del settecento e alla quale il Kant faceva compiere un gran passo con la critica dell'intuizione come concetto confuso (che era la dottrina leibniziana), e un altro gran passo lo Schelling e lo Hegel, i quali, collocando l'arte tra le pure forme dello spirito, si riavvicinarono al Vico. E il romanticismo (specie tedesco, ma anche piú o meno quello degli altri paesi), in quanto si espresse in formole teoriche, fu vichiano, perché celebrazione della fantasia come originale potenza creatrice. Ricorsero le sue dottrine sul linguaggio, interpetrato non piú intellettualisticamente quale sistema artificioso di segni, ma come libera e poetica cręazione dello spirito dallo Herder e dallo Humboldt. La dottrina della religione e del mito, dopo che ebbe abbandonato l'allegorismo e la teoria della frode, riconobbe con David Hume che la religione è un processo naturale, corrispondente agl'inizî della vita umana, tutti passione e immaginazione; con lo Heyne, che il mito è un « sermo symbolicus », non prodotto da arbitrio ma da bisogno e povertà, dalla «sermonis egestas », la quale si esprime "per rerum iam tum notarum similitudines "; con Ottofredo Yüller, che è impossibile intendere il mito senza rientrare nell' intimo dell'anima umana, dove se ne scorge la necessità e la spontaneità. La religione non fu piú guardata eome qualcosa di estraneo o di nemico verso la filosofia, come stoltezza o come inganno di gente furba alle spalle di gente semplice, ma, come il Vico voleva, quale filosofia rudimentale, onde tanto si trova nella metafisica ragionata quanto era gia in qualche modo nella metafisica poetica o religiosa. Similmente, poesia e storia non furono piú tenute disgiunte o contrapposte perché si combattessero a vicenda; e come già uno dei grandi ispiratori della nuova letteratura tedesca, lo Hamann (per tanti rispetti simile al Vico nelle tendenze, benché impari nella potenza mentale), aveva ammonito e preveduto dicendo: "se la nostra 
pocsia non vale, anche la nostra storia si fara piú magra delle vacche di Faraone », un alito di poesia ravvivò la storiografia del secolo decimonono, la quale da incolore ridivenue pittoresca, da fredda che era acquistò calore e vita. La critica all'utilitarismo degli Hobbes \& dei Locke, e l'affermazione della coscienza morale come spontaneo pudore e giudizio senza alcuna riflessione, si ripresentò, bene armata, nella Critica della ragion pratica; e la polemica contro l'atomismo sociale e il conseguente contrattualismo, nella Filosofia del diritto hegeliana. La libertà di coscienza e l'indifferentismo religioso, professati e inculcati dai pubblicisti del seicento, fu negata come dottrina filosofica; e un popolo senza Dio sembrò allo Hegel, come già al Vico, jutrovabile nella storia ed esistente solo nelle panzane dei narratori di viaggi in paesi ignoti o poco noti. Continuando l'opera della Riforma, che il Vico non aveva potuto conoscere né esattamente giudicare, la filosofia idealistica germanica intese non gia a strappare dalla vita la religione, ma ad affinarla, dando valore spirituale di religione alla filosofia stessa. Il certo, il duro certo, che il Vico distingueva dal vero del diritto, formo tema di meditazioni dal Tomasio al Kant e al Fichte, e da costoro ai recentissimi che cercarono, se anche non trovarono, il criterio distintivo tra le due forme, e tutti o quasi tutti mostrarono viva coscienza di cio che chiamarono « forza * o « coazione » e che era stato come obliato nella vecchia, superficiale e rettorica dottrina moralistica. Isa scuola storica del diritto, reazione al rivoluzionarismo e al riformismo astratto del secolo decimottavo, doveva ripigliare l'altra polemica del Vico contro il platonismo o il grozianismo di una repubblica ideale o di un diritto naturale, fuori e sopra la storia e misura della storia, e riconoscere col Vico che il diritto è corrispettivo all'intera vita socialc di un popolo in un dato momento storico e giudicabile solo 
in relazione a questa, e vivo e plastico e in continua mutazione come il linguaggio. Finalmente, la Provvidenza vichiana, cioè la razionalita e oggettivita della storia, che osserva logica diversa da quella che le viene attribuita dalle individuali immaginazioni e illusioni, prese un nome piú prosaico, ma non mutò carattere, nell'astuzia della ragione, formolata dallo $\mathrm{Hegel}$; e fu spiritosamente e cervelloticamente ritradotta nella popolare astuzia della specie dello Schopenhauer, e, poco spiritosamente sebbene assai psicologicamente, nella cosí detta legge wundtiana dell'eterogenesi dei fini.

Sono, come si vede, quasi tutte le idee capitali della filosofia idealistica del secolo decimonono, che si possono considerare ricorsi di dottrine vichiane. Quasi tutte, perché ve n'ha poi qualcuna della quale nel Vico si trova non il precorrimento ma l'esigenza, non l'addentellato $\mathrm{ma}$ la lacuna da riempire; e per questa parte si ha non piú il ricorso ma il progresso del secolo decimonono sopra di lui, e dal coro gli sorgono contro voci discordanti di antmonimento o di rimprovero. La distinzione vichiana dei due mondi dello spirito e della natura, a entrambi i quali era applicabile il criterio gnoseologico della conversione tra vero e fatto, ma al primo applicabile dall'uomo stesso perché quel mondo è opera dell'uomo, e perciò da lui conoscibile, e al secondo da Dio creatore, e perciò inconoscibile all'uomo, non fu accettata dalla nuova filosofia che, piú vichiana del Vico, dell'uomo semidio fece Dio, sollevò la mente umana a spirito universale o Idea, e la natura spiritualizzò e idealizzò e, come prodotto anch'essa dello spirito, tentò d'intendere speculativamente nella * Filosofia della natura. Distrutto per tal modo questo residuo di trascendenza, rifulse il concetto di progresso che il Vico non aveva scorto, e che i cartesiani e i loro seguaci del secolo decimottavo, nella loro maniera superficialmente ria- 
zionalistica, avevano pure in qualche modo intravisto e affermato.

Ia a questo mancato riscontro fa compenso quello pienissimo tra le « discoperte » storiche vichiane e la critica e storiografia del secolo decimonono. Riscontro, anzitutto, nei canoni metodici: nella scepsi circa i racconti degli storici antichi, nella superiorita riconosciuta ai documenti e monumenti sulle narrazioni, nell'indagamento dei linguaggi come tesori dei concetti e dei costumi primitivi, nell'interpetrazione sociale dei miti, nell'importanza data agli svolgimenti spontanei sulle estrinseche comunicazioni delle civiltà, nell'avvertenza di non interpetrare la psicologia primitiva con la psicologia moderna; e via discorrendo. Riscontro nelle soluzioni effettive dei problemi storici, perché furono riaffermati il carattere areaico e barbarico delle civilta primitive greca e romana, e l'indole aristocratica e feudale delle loro costituzioni politiche; il cerimoniale dell'antica giurisprudenza riapparve come un poema drammatico, allusivo ad azioni guerresche; il travestimento degli eroi romani in eroi democratici ebbe termine coi giacobini francesi e coi loro imitatori in Italia e altrove; Omero fu considerato poeta tanto piú grande quanto piú rude; la storia di Roma venne ricostruita soprattutto sulla base del diritto romano, e i nomi dei sette l'e apparvero simboli di istituzioni e le origini tradizionali di Roma tarde invenzioni greche o condotte sui modelli greci; la sostanza di quella storia fu riposta nella lotta economica e giuridica di patriziato e plebe, e la plebe derivata dai famoli o clienti; la lotta di classe, che il Vico pel primo aveva rischiarata di cruda luce, fu accolta come criterio di larga applicazione alla storia di tutti i tempi e per l'intelligenza dei maggiori rivolgimenti sociali; il Medioevo, segnatamente durante la restaurazione seguita al periodo napolconico, attrasse gli animi e le menti, vagheg- 
giato e sospirato come il contrapposto della societa razionalistica e borghese e inteso, percio, come il periodo religioso, aristucratico e poetico che il Vico aveva scoperto, come l'età giovanile dell' Europa moderna. L'Italia ritrovò cosi la grandezza del suo Dante, e quella critica dantesca, che il Vico aveva iniziata, il De Sanctis portò innanzi: come il Niebuhr e il Mommsen maturarono la storiografia romana di lui; il Wolf e i posteriori critici di Omero (particolarmente Ottofredo Müller), la teoria omerica; il Cornewal Lewis, il Pais e il Lambert, l'altra e analoga sua teoria circa la legge delle dodici Tavole; lo Heyne, il Müller, il Bachhofen, l'interpetrazione dei miti; il Grimm e altri filologi, il desiderato di una ricostruzione della vita primitiva per mezzo delle etimologie; il Savigny e la scuola storica, lo studio degli svolgimenti spontanei del diritto, con preferenza data alle consuetudini sulle leggi e i codici; il Thierry e il Fustel de Coulanges in Francia, il Troya in Italia e una legione di dotti in Germania, la concezione del medioevo e del feudalismo; il Marx e il Sorel, l'idea della lotta di classe e dei ringiovanimenti delle società per mezzo di ritorni a stati d'animo primitivi e a ricorsi di barbarie; perfino il superuomo del Nietzsche rinnovò, in qualche modo, l'eroe vichiano. Sono alcuni nomi soltanto, che ricordiamo alla rinfusa e un po' a caso, perché a ricordarli tutti e ciascuno a suo luogo si dovrebbe scrivere l' intera storia del pensiero europeo nella sua fase più moderna: una storia che non è ancora cliusa, sebbene abbia avuto, sotto nome di a positivismo », una parentesi di parziale ritorno all'astrattismo e materialismo del secolo decimottavo, che essa, invece, sembra ora chiusa fermamente.

Questi molteplici ricorsi dell'opera di un individuo ncll'opera di piú generazioni, questo riscontro tra un individuo e un secolo, giustificano una definizione immaginosa 
che si pùo dare del Vico, desumendola dallo svolgimento posteriore: che egli fu né piú né meno che il secolo decimonono in germe. Definizione che può valere come compendio della nostra ricostruzione ed esposizione delle dottrine di lui, e contribuire a far intendere il posto che gli spetta nella storia della filosofia moderna. Egli è da collocare veramente accanto a quel Leibniz, al quale è stato spesso paragonato: da collocarvelo non perché somigli al Leibniz, come si è creduto (i paragoni istituiti a quest'intento si provano o falsi o superticiali), ma, anzi, perché non gli somiglia punto o gli è quasi opposto. I1 Leibniz è il potenziamento del cartesianismo: intellettualista, nonostante le piccole percezioni c la conoscenza confusa; meccanista, nonostante il dinamismo, che è forse piú nella sua immaginazione che nel suo effettivo pensiero; antistorico, nonostante la sua immensa erudizione storica; chiuso a ogni coscienza di quel che sia il linguaggio, nonostante che del linguaggio si occupasse fervidamente durante tutta la vita; antidialettico, nonostante la tentata spiegazione del male nell'universo. Rispetto all'jdealismo posteriore, la filosofia leibniziana sta come l'espressione piú perfetta della vecchia metafisica, che doveva essere superata; quella del Vico, come l'abbozzo della nuova metafisica, che doveva essere svolto e determinato. L'uno si rivolse al suo secolo, che gli si affollò intorno e ne propagò rapidamente e sonoramente la voce; l'altro, a un sceolo avvenire ed cbbe intorno a sé il deserto e il silenzio. Ma la folla o il deserto non aggiungono o tolgono nulla al carattere intrinseco di un pensiero. 
.

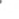




\section{LeOghI DEL VICO ADOPRATI O RICHIAMATI NEL CORSO DELL' ESPOSIZIONE}

I. - Per questo capitolo si vedano il De studiorum ratione, il Le antiquissima italorum sapientia, le dne Risposte al "Giornale dé letterati a e la prima parte dell'Autobiografia. Per l'accenno allo spirito della Riforma (p. 8), Opere, ediz. Ferrari seconda, YI, 5.

II. - Genesi del nuovo orientamento gnoseologico del Vico (p. 22), Opere, ediz. cit., IV, $366^{\circ} \mathrm{sgg.;}$ - la certezza della storia (p. 23), V, 147; - il mondo della natura e il mondo umano (p. 24), IV, 33; V, 136-7; - sillogismo, sorite, metodo cartesiano, induzione baconiana (p. 25), V, 239 ; - l'asserito " metodo geometrico " (lella Scienza nuova e suo vero significato (pp. 25-6), V, 51; VI, $99 ; \mathrm{V}, 51 ;-$ significato del detto che per pensare la Scienza nuova convenga * ridursi a uno stato di somma ignoranza» $\theta$ « di puro intendimento (pp. 26-7), IV $33 ; \mathrm{V}, 136,50,51$; - l'* immaginato mondo di linee e di numeri " della matematica e la realtà (p. 29); II, 118, 160; - in qual guisa la scienza del mondo umano proceda come la geometria (pp. 29-30), V, 147; - la « divinità delle lrove della Scienza nuova (p. 30), ivi; - la filosofia e la filologia (pp. 30-1), III, 200; - l'autorità e la ragione (p. 31), V, 171 sgg.; - la filologia studia non solo le parole ma anche le cose (ivi), V, 115, 149 ; - oggetto amplissimo della filologia, IV, 27; V, 98; - filosofia e filologia ancora estranee l'una all'altra ai tempi del Vico (pp. 31-2), IV, 20 sgrg.; - l'erudizione di Grozio non è intrinseca al suo argomento (p. 32), IV, 19; - il disprezzo di Cartesio e del Male- 
branche contro l'erudizione (ivi), III, 232; - la necessità di ridurre la filologia a scienza (ivi), III, 233; IV, 307; V, 98, 174; - la filologia è necessaria conseguenza della filosofia (p. 33), III, 200; il metodo baconiano (ivi), V, 103; - l'esigenza d'una storia tipica delle società umane (ivi), V, 149-50, 174; IV, 20, 248; - il titolo " invidioso " della Scienza nuova (p. 34), V, 562.

III. - Varî significati della " storia ideale eterna "vichiana (p. 40-1), Opere, V, 147; - la * degnità » sulla * natura delle cose (1. 41), V, 99 (degn. XIV); - le cosí dette " prove metafisiche " vichiane (p. 42), IV, 81; - i fatti dubbî sono asseriti dal Vico " in conformità delle leggi » e ritenuti da lui * verità meditate in idea 》(ivi), IV, 73; V, 91, 148-50.

IV. - II « certo" (p. 45), Opere, V, 97; - la " sapienza poetica " costituisce "quasi tutto il corpo" della seconda Scienz" nuova (ivi), V, 44 ; - le " aspre difficultà " per discendere dalle nostre nature ingentilite a quelle degli nomini primitivi e l'idea della * natura simpatetiea »(p.46-7), IV, 36; V, 141, 166; - una delle ragioni perché " nuova : la Scienza del Vico (p. 47), V, 42; errori di Platone, Giulio Cesare Scaligero, Franceseo Sanchez, Gaspare Schopp (ivi), III, 232-3; IV, 194, 228; - gli errori di Grozio, Selden e Puffendorf (ivi), IV, 20; V, 175; - il Vico confessa l'errore commesso da lui medesimo nel De antiquissima (ivi), IV, 194, 228; - la sapienza poetica è la * chiave maestra " della seconda Scienza nuova (ivi), V, 42; - la « Logica poetica (p. 48), V, 179; la sapienza riposta fu intrusa nella poesia dai filosofi, III, IV, V, pressim, e cfr. per es. IV, 191-3; - la poesia è * necessità di natura " e la « prima operazione della mente umana » (p. 49), V, 220,237 ; - l'uomo, prima di riflettere, avverte con animo commosso e, prima di articolare, canta (ivi), V, 112, 114; - la poesia è anteriore alla prosa (ivi), V, 115; - il linguaggio a proprio " e il linguaggio « improprio "(ivi), V, 186; - la poesia e la metafisica (ivi), IV, 199-200; - nessuno fu insieme gran metafisico e gran poeta (ivi), IV, $200 ; \mathrm{V}, 441$; - i poeti sono il senso, i filosofi l'intelletto dell'umanità (pp. 49-50), V, 152; - il linguaggio ler " atti muti » (p. 50), V, 197, 466; - le ling'uo articolate non sono per convenzione (pp. 50-1), V, 209-10; - le origini delle lingue furon trovate dal Vico nei principî della poesia (p. 51), V, 
194-218, 466-7; - una e medesima è l'origine del linguaggio e (lella scrittura (ivi), V, 198; - i geroglifici (ivi), V, 201, 249; identità tra farola (poetica) ed espressione (p. 52), IV, 205; - le cinque « parole reali \$ d'Idantura (ivi), V, 82, 201-2; - gli alti Irapaveri troncati dal re Tarquinio (ivi), V, 202; - analoghi procedimenti espressivi presso popolazioni selvagge e i volghi (ivi), V, 202-3; - le imprese, bandiere, medaglie, monete (ivi), IV, 202$16 ; \mathrm{V}, 230-1 ;-\mathrm{la}$ * favoletta " sull'origine delle imprese (ivi), IV, 206; - le imprese primitive furon * mutole " (ivi), V, 230-1; le insegne e bandiere sono una sorta di " lingua armata (ivi), IV, 211-2; - le teorie di Platone, Aristotele, ecc. sulla poesia son « rovesciate»da quella del Vico (p. 53), IV, 163; V, 169; - i " canti degli uccelli » e il " sibilo dei vonti » del Patrizzi (ivi), III, 27.; - le teorie di Platone, Lazio, Scaligero, Sanchez sull'orisine delle lingue sono insoddisfacenti (ivi), IV, 194; - saggio di opinioni « boriose " e " ridevoli " sull'origine della scrittura (ivi), $\mathrm{V}, 195-7$; - polemica contro i trattatisti delle imprese eroiche (ivi), IV , 202; V, 230; - la poesia rappresenta il vero nella sua idea ottima (p. 54), IV, 224; - ha per fine l'animazione dell' inanimato (ivi), IV, 163; - non ̇̀ altro che imitazione (pp. 54-5), V, 112, 23s; - ha per propria materia l'impossibile credibile (p. 55 ), III, 272; IV , 165; V, 168-9; -- è nata da inopia (ivi), III, 271-2; V, 220, 442.3; - identita del vero dei filosofi e del vero dei poeti (ivi), V, 164; - da inopia nacquero il canto e lo metafore (ivi), $\mathrm{V}, 220 \cdot 1$; - scopo della poesia è insegnare al volgo l'operare virtuosamente (ivi), V, 16t; - diverse forme della poesia (p. 56), V, 456 sg.;.; - del verso e del metro (ivi), V, 222; - del parlare figurato (ivi), V, $182 \mathrm{sgg.;}$ - delle parti del discoi'so (ivi), V, 213 sgg.; - un caso d'afasia osservato dal Vico a Napoli (ivi), V, „17; - identità intrinseca tra pittura e poesia (ivi), VI, 40; analogie tra la poesia e la pittura medievali (ivi), III, 270; V, 438; - le delicatezze delle arti (belle) son frutto delle filosofie (ivi), V, 55; - la prima età del mondo fu tutta occupata intorno alla prima operazione della mente (p. 57), V, 237; - le arti (meccaniche) sono poesie in certo modo reali (ivi), $\mathrm{T}, 112,288$; - il diritto romano è un e poema drammatico serioso * (ivi), III, 347; IV $225 ; \mathrm{V}, 531 ;-$ la poesia è considerata dal Vico come teolo- 
gia fantastica (pp. 万̃ -8), V, 162 sgg.; - come educatrice di volgo (p. 58), V, 253 sgg.; - come fisica immaginaria (ivi), V, 369 sgg.; i popoli primitivi furon tutti di sublimi poeti (p. 58), V, 107, 443; poeti sono pol Vico perfino i fanciulli (ivi), V, 112; - gli uomini primitivi eran nulla o assai poco ragione (p. 59), V, 379; - quasi tutto corpo (ivi), V, 440; - la lingua degli dèi fu quasi tutta muta (ivi), V, 212; - l'immagine dei " grandi rapidi fiumi » (ivi), $\mathrm{V}$, 187 ; - i poeti primitivi furon fantastici per natura, quelli di epoche incivilite son tali per industria (ivi), III, 274; VI, 37; come il Vico ponesse il problema dell'origine della civiltà (p. 60). IV, 24.

V. - Il De sapientia reterm di Bacone è più ingegnoso che vero (p. 64), Opere, IV , 360, 365; - il De theologia gentili del Voss (ivi). IV, 21; V, 171; - la Demonstratio evangelica dell'Huet e il Phaleg et Chanaan del Bochart (ivi), V, 80; - i miti non contengono sapienza riposta (ivi), V, 169; - hanno significati univoci (p. 65), IV, 169; V, 43, 111-2; - il mito di Giove fu efrettivamente creduto dagli uomini primitivi (ivi), $\mathrm{V}, 166$; - i miti sono storia vera foggiata da spiriti primitivi (ivi), IV, $74 ; \mathrm{V}, 18,109$; - la sapienza riposta fu intrusa in essi soltanto in tempi colti (ivi), IV, 191-3; - né poi vennero trasmessi da un popolo all' altro (ivi), V, 80, 81; - il mito d'Ercole (pp. 66-7), IV, 167; - il mito del Cielo (p. 67), IV, 164: $\mathrm{V}, 248$; - il Vico asserisce, sí, clie i miti contengono i sensi storici. non filosofici, dei popoli primitivi (p. 68), V, 43; - ma propriamente egli ruol contrapporre $i$ sensi storici a " sensi mistici di altissima filosofia " e ai sensi * analogi ", intrusi in tempi colti nei miti (jvi), V, 169; - come la mente umana si liberi dai miti (pp. 68-9), IV, 172; V, 186, 411, 220; - definizione richiana dell'errore (p. 69), III, 110; II, 96; V, 166; - e del falso (ivi), IV, 169 ; - ogni favola ha un motivo di rero (ivi), IV, 169; V, 110;i miti sono embrione del sapere riposto (ivi), V, 420; - i creatori dei niti ("poeti teologi ") furono il senso, i filosofi l'intelletto dell'nmanità (ivi), $\mathrm{V}, 421$; - come si sia svolta storicamente l'idea di Dio (pp. 69-70), IV, 50-1; - i concetti delle tre pene divine $\theta$ delle vie purgativa e unitiva furono ispirati a Platone dalle favole (p. 70), V, 387; - donde derivino gli esempî esopici, l'induzione socrática e il sillogismo aristotelico (ivi), V, 192; - 
l'etimologia di « olfacere »(ivi), V, 379; - due pensieri circolano nella Scienza nuova sui rapporti tra religiono e filosofia (pp. 70-1), $\mathrm{V}, 165,168$; - l'origine della divinazione (p. 71), V, 168; - il De origine idolatrice $\theta$ il De oraculis ethnicorum del van Dale (ivi), $\mathrm{V}, 171$; - le religioni sono nate non da impostura d'altrui ma da propria credulita (ivi), V, 108; - la religione precedo la filosofia $\theta$ il linguaggio (p. T2), IV, 193-4; - teologia poetica, naturale o rivelata (p. 72 ), V, 155 ; - il popolo ebraico fu privilegiato da Dio (ivi): efr. piú giú le citazioni al eap. XIII.

VI. - Poesia e storia (p. 75), Opere, V, 437, 109, 441; - insistenza del Vico nel notare che l'elemento poetico è intrinseco alla storia (ivi), V, 109, 110, 444; VI, 15; - lo stile di Erodoto (ivi), V, 18; - il detto di Cicerone (ivi), IV, 400; - le istituzioni sociali e la filosofia (p. 76), V, 532, 534; - i * quattro auttori * del Vico (pp. 76-7): Platone e Tacito, IV, 351; Pacone, IV, 351-2; Grozio, IV, 366-7; - l'insistente polemica del Vico contro Grozio, Selden, Puffendorf e gli « adornatori s del sistema groziano (pp. 76-7), III, passim, spec. 11-2; IV, passim, spec. 16-20, 307-8, 391; $\mathrm{V}$, passim, spec. 6, 175-7; - la polemica contro gli utilitaristi (p. 80): Hobbes, Spinoza, Bayle, Machiavelli, III, 12; Loeke, IV, 340 ; V, 49; epicurei e stoici, IV, 14; il "ve victis " del brenno gallico, III, 57 ; - il fallimento dello « sforzo magnanimo * dell'Hobbes (pp. 80-1), V, 106 ; - - del Locko (p. 81), VI, 5; - lo Spinoza e gli « spinosisti » dell'anticlità (ivi), V, 138, 49; - la polemiea contro i trattatisti di etica dal Cinque al Settecento (pp. 81-2): Cartesio, IV, 343; Malebranche e Pascal, VI, 127; Nieole, Pallavicini e Muratori, VI, 98; - l'utilità e l'honestas (p. 82), III, 30; - la frodo e la forza (ivi), IV, 87; III, 57; - la fedo nello promesse ò base della societa, e polemica relativa contro il Loeke (pp. 82-3), IV, 39-40; - l'utilità è oceasione, non causa (p. 83), III, 30 ; -- il detto di Pomponio (ivi), III, 30; IV, passim; V, passim;-l'omicidio per legittima difesa e il furto per fame (ivi), III, 31; l'etica platonica e l'etica aristotelica (pp. 83-4), IV, 334-5; - l'etiea dei ginreconsulti romani (p. 84), IV, 334; - i titoli magnifici dei libri dei ginsnaturalisti non eorrispondono al loro contenuto (ivi), IV, 35; - i principî di Grozio pesati con la bilancia della critiea non appariseono necessarî (ivi), III, 12; - Grozio e la questione 
dell'utilità (ivi), III, 30; - Grozio e la disputa se vi sia diritto in natura (ivi), V, 97; - Grozio non dinostrò la sua ipotesi sui " somplicioni > (ivi), IV , 43; - e fu epicureista senz'avvedersene (ivi), IV, 17; - la a degnità * vichiana sulle « $\cos \theta$ fuori del loro stato naturale » (pp. 84-5), V, 97 (degn. VIII); - il rimorso e il pudore son fonti di tutte le virtú (p. 85), III, 234 sgg.; IV, 49-50; il * senso comune degli uomini d'intorno allo umane necessità * è fonte del diritto delle genti (ivi), V, 98 (degn. XI e XII); - i costumi son generati dall'interno, non dall'esterno (ivi), V, 131; l'arbitrio umano è incertissimo (ivi), V, 98.

VII. - Il cielo fulminante fa immaginare ai * bestioni » primitivi una divinità corrucciata (p. 87), Opere, V, 165-8, 106, 142; per placarla essi raffrenano le passioni (ivi), V, 242; - il « conato » (p. 88), V, 142, 172-3; - il timore di Dio è fondamento della vita umana (ivi), IV , 291; V, 242-3, 250; - l'origine del culto religioso, dei matrimonî, delle sepolture (ivi), V, 137; - la religione è il solo mezzo per ridurre i popoli infieriti con le armi (ivi), $V$, 106; - la pietà è insegnata ai fanciulli mediante il timor di Dio (ivi), V, 242; - l'uomo disperato desidera un essere superiore che lo salvi (ivi), $\mathrm{V}, 1+2$; - tutte le nazioni credono in una divinità provvidente (ivi), $\mathrm{V}, 138$; - popoli senza Dio sono novelle di viaggiatori (ivi), V, 137; - parallelo tra le cose fisiche e le cose morali (pp. 88-9), IV, 17; - spenta la coscienza religiosa, si spegne il concetto di società e stato (p. 89), III, 387; - il concetto della divinità presso gli ebrei, i cristiani, i gentili e i maomettani (ivi), V, 138; - gli epicurei e gli stoici e il detto di Cicerone a Tito Pomponio Attico (ivi), ivi; - l'Hobbes e lo Spinoza - la loro inintelligenza del vero concetto di societa e stato (ivi), IV, $320 ; \mathrm{V}, 106,136,138$; - Pietro Bayle e i dotti della * sfumata letteratura ( (ivi), V, 290; - polemica contro Grozio (ivi), IV, 17; V, 176; IV, 43; - contro Puffendorf (pp. 89-90), IV, 18; $\mathrm{V}, 176$; - la religione vale come concezione della realtà, o che si affermi come metafisica intelligibile (p. 90), V, 153-4, 241-2; o che come metafisica poetica (p. 91), V, 163; - la spaventosità delle religioni primitive e polemica relativa contro Plutarco (ivi), V, 253; - il vero significato del detto che * il timoro feco gli dèi (ivi), V, 108; - un senso comune d'immortalità è nascosto 
nella mente degli uomini (ivi), IV, 9; - cómpito della filosofa ̀̀ talora, pol Vico, sollęvare l'uomo caduto (pp. 91-2), V, 96, 154; - ina tal'altra la filosofia, secondo lui, è adatta piuttosto a ragionare (p. 92), V, 242; - e la morale dei filosofi, piú che alla pratica, giova all'eloquenza (ivi), V, 573-4; - la religione caratterizza l'epoca barbarica, la filosofia i tempi civili (ivi), V, 490; il detto attribuito dal Vico a Polibio (ivi), V, 106, 137-8, 534; la religione ridusse $\mathrm{i}$ * figliuoli dei Polifemi * all'umanità (ivi), V, 108; - come il Vico concepisse lo * stato ferino * (p. 93), V, 142; - come il libero arbitrio (ivi), V, 97; - quale posizione assumesse nelle controversie sulla grazia (pp). 93-4), IV, 332-3; la grazia naturale e la soprannaturale (p. 92), V, 97; - la polemica contro il Selden (pp. 92-3), IV, 17; V, 176; - gli ebrei e le restanti nazioni, e il diverso aiuto loro prestato rispettivamente dalla provvidenza (p. 95), V, 131, e cfr. piú giú le citazioni al cap. XIII.

VIII. - Dio è * posse, nosse. velle infinitum », l'uomo " $p . n$. v. finitum * (p. 97), Opere, III, 21, 22; - lo Stato è immagine di Dio (ivi), III, 70-1; - definizione della giustizia come rettrice di egualità e sua distinzione in commutativa e distributiva (ivi), III, 28; IV, 331 ; - l'egualità e i meriti (p. 98), IV, 46; - la teoria del Vico sul riso (ivi), IV, 309-13; - il Vico annulla egli medesimo le distinzioni tra le due giustizie, le tre virtú e i tre diritti (ivi), III, 46, 27, 40; - tutt'uno fanno per lui anche giustizia e virtú (ivi), III, 28; -- la dottrina vichiana della pena (p. 99), III, 43-6; - la morale degli epicurei (ivi), IV, 335; - e degli stoici (ivi), IV, 14; - il griansenismo (pp. 99-100), VI, 5; - i filosofi "solitari " e i filosofi « politici », e l'eccellenza della dottrina dei platonici sulle passioni (p. 100), V, 96, 97; - lo utilità non sono né buone né cattive (ivi), III, 30; - il ius naturale prizs e il i. n. posterius, e polemica relativa contro Grozio (ivi), III, 49-52; il certo, il vero, l'autorita, la ragione (p. 101), III, 53; - la Scienza nuova contiene, pel Vico, una filosofia dell'autorita (ivi), V, 148, 171; - la lettera delle leggi (ivi), III, 53; - il " certo delle leggi "(ivi), V, 133; - le prime leggi furono exempla (p. 102), $\mathrm{V}, 240-1$; - il diritto primitivo fu concepito come forza materiale (ivi), IV , 45, 46; - i "fortissimi ", per altro, erano anche etica- 
mente gli " optimi ", III, 65, 63; - il lore diritto era il certo "ex vero mixtum" (ivi), III, 87 ; - male Grozio e gli altri giusnaturalisti han cominciato la storia dell'umanità dalla metà in giú (p. 103), IV, 20, 18; V, 175; - polemica contro Hobbes, Machiavelli ed Epicuro (ivi), IV, 171; - il quarto " auttore" del Vico: Tacito, e eome da Tacito e da Platone egli fosse indotto a meditare una storia eterna (pp. 103-4), IV, 351; - la "repubblica" di Platone e la “feccia " di Romolo (p. 104), V, 96.

IX. - Il sentire senz'avvertire, l'avvertire con animo commosso, il riflettere con mente pura (p. 105), Opere, V, 112; -- tutte le manifestazioni della vita dell'umanità si corrispondono nei tre tipi sociali del « ferino * (divino), del \& barbarico (eroico) e del \& ci vile * (umano) (pp. 105-6), V, 462-3; IV, 44, o cfr. passim tutto il quarto libro della seconda Sciena a mova; - le forme miste vaghes"giate da taluni politici son mostri (p. 106), Opere, V, 511; - i governi debbono esser conformi alla natura degli uomini governati; la scuola dei principi è la morale dei popoli (ivi), IV, 73; V, 117; Sciensa nuova seconda, ediz. Nicolini, p. 115S; - la necessità o conseguente bontà d'una società primitiva fondata sulla guerra e sul diritto del piú forte (ivi), Opere, IV, 46; - l'eguale necessita e bontì del tipo di società fiorente nei tempi della ragione umana tutta spiegata (ivi), IV , $46 ; \mathrm{V}, 466$; - le varie età della vita individuale e quello della vita delle nazioni (pp. 106-7), III, 192-3; il ius naturale philosophorum è ammesso dal Vico a parole (p. 107), III, 86; - ma negato nel fatto, allorché egli lo identifica col diritto eroico convertito in quello ingentilito e polemizza al riguardo contro Grozio (pp. 107-8), IV, 18; V, 148, 176; - analogamente, il Vico confessa d'essere stato indotto dallo studio di Platone a meditare un * diritto ideale eterno » (p. 108), IV, 335; - ma siffatto diritto egli nega, allorehé polemizza contro il medesime Platone, perché non tenne eonto dei « bestioni tutti stupore e ferocia * e meditò una repubblica ideale "onde le nazioni non si reggono punto (pp. 108-9), IV, 15; - la "generis humani respublica " ovvero la « gran città delle nazioni fondata e governata da Dio" è nient'altro che la Storia (pp. 109-10), III, 55; V, 571; - i costumi si svolgono gradualmento (p. 110), V, 117; - le due « praticho assegnate dal Vico alla sua Seienza nella prima Scienza 
nuove (pp. 110-1), IV, 24-5, 13, 159-60; - il paragrafo sulla " pratica ", aggiunto in una redazione intermedia della seconda Sciensa nuova e soppresso in quella definitiva (pp. 112-3), Sciensa nuova secondu, ediz. Nicolini, p. 1053 sgg.

x. - La Provvidenza va ragionata sull' \& econonia delle cose civili $\gg$ (p. 115), Opere, $\mathrm{V}, 13,143-4 ;$ - la sapienza poetica è cominciata dalla contemplazione d'un Dio provvidente (p. 110), V, 168; - insistente critica dol Vico della fortuna, del fato e del caso (p. 120), II, 107-8; III, 211-2, ecc.; - gli stoici e la \& catena di Giove" (ivi), V, 172; - le opportunita, le occasioni e gli accidenti (pp. 120-1), III, 22; - quello che fece il mondo delle nazioni fu Mente, non Fato o Caso (p. 21), V, 572; - esempî di illusioni unane circa i fini delle azioni che si compiono (pp. 121-2), IV, 34-5; V, 14, 333-5, 572; - l'utilitarismo in una pagina del Vico (pp. 122-3), V, 113; - cosa fosse la pubblica virtú romana (p. 123), V, 45-6; - la divinità provvidente è l'unitì dello spirito che dà vita al mondo delle nazioni (ivi), V, 463; - il problema del male in uno dei primi scritti del Vico (p. 124), II, 107.

XI. - La \& barbarie della riflessione " (p. 127), opere, V, 536. $570 ;-$ la successione delle forme di governo e polemica relativa contro Polibio (p. 128), 1V, 62; - la tradizione egiziana sulle tre età degli dèi, degli eroi o degli uomini (p. 127), IV, 251; V, 60, 105, 198; - quel che il Vico trovava nella storia romana (ivi): cfr. piú giú le citazioni al cap. XVII; - le « degnità > formolanti le leggi della dinamica sociale (pp. 129-30), V, 116- 7 (degn. LXVI e LXVIII); - perché Cartagine, Capua e Numanzia non seguirono il corso unifor'me delle nazioni (pp. 130-1), V, 558; - perché non l'han seguíto nemmeno gli americani (p. 131), V, 561; - le ancora " aristocratiche "Inghilterra e Polonia diverranno un giorno perfettissime monarchie (ivi), V, 559; - il modio evo s'apre con lo stabilimento del cristianesino (ivi), V, 537; - le due vio offerte alle nazioni corrotte: il ritorno allo stato ferino e il dominio stra. niero (ivi), V, 570; - uomini di stato e fllosofi, lavorando di conserva, posson contencre la dissoluzione d'una nazione (ivi), IV, 13 ; - le pocho republuliche aristocraticho persistenti nel secolo decimottavo si conservano con arti di sopraffina sapienza (ivi), V, $557-8$; - una « compiuta umanità " sembra al Vico che sia sparsa, 
nei principî del secolo decimottavo, per tut́e le nazioni (pp. 131-2), V, 559-61; - una canzone giovanile del Vico cupamente pessimistica (p. 133), VI, 317-21 (Affetti di un disperato); - il triste quadro dato dal Vico della condizione degli studi ai suoi tempi (ivi), VI, 3-1T, e cfr. piú giú il capitolo XIX; - " Iundus iuvenescit », ecc. (ivi), VI, 127, o cfr. VI, 35; Sciense nuora seconde, ediz. Nicolini, pp. 348-55; - i climi, le disposizioni delle razze e simili son considerati talora dal Vico come fattori storici (p. 135), Opere, III, 95, 266; IV, 73, 249-50; - né egli manca d'osservare che la scrittura alfabetica fu trasmessa dai caldei ai fenici o da questi agli egiziani (p. 136), V, 206.

XII. - Per questo capitolo, vedere le stesse fonti indicate pel capitolo I, e cfr. Opere, VI, 105-6; V, 524-5.

XIII. - Contrapposiziono degli ebrei alle genti e della storia sacra a quella profana (pp. 146-7), Opere, III, jassim, specialmente 206, 207, 262-5, 342; IV, pessim, spec. 51, 74-5, 76-7, 91, 151-2, 182, 252; V, passim, spec. 113, 131, 148, 229; - Tacito chiama gli ebrei * uomini insocieroli * (pp. 150-1), IV, 17, e cfr. V, 7981 ; - il privilegio degli ebrei di serbare intatte le loro memorie fin dal principio del mondo (p. 151), III, 303; IV, 23; V, 60, 61, 103-4; - la « perpetuità della storia saeja con la profana $\gg$ (p. 148), III, 249-51; IV, 253; - la bizzarra costruzione fatta dal Vico dei cominciamenti della storia umana (pp. 152-3), III, 249-61; IV, 7580 ; V, 108-9, 157-61; - saggio dei tentativi vichiani di apologetica biblica (pp. 153-4): 1. prove del diluvio universale e dell'esistenza dei giganti, III, 255-7; IV, 78-9, 289; V, 104-5; 2. il governo teocratico fu peculiare agli ebrei, III, 249, 362; 3 . a loro fu ignota la divinazione, III, 207; V, 104; 4. nei verbi ebraici la terza persona singolare maschile del perfetto corrisponde alla radice, V, 217-8; - nella Scienza nuova si ha tutta spiegata la storia " sull'identità in sostanza d'intendersi e diversità dei modi di spiegarsi ( (p. 155), V, 562; - i fatti, in essa, si arrecano soltanto come esempî (ivi), IV, 38; - la diligenza è minuta e tarda virtú (p. 157), Scieña nuovce seconda, ediz. Nicolini, pp. 11-2.

XIV. - Le magnifiche opinioni intorno all'antichità; l'uomo rovesciato nell'ignoranza fa di sé regola dell'universo; "fama crescit eundo "; " onne ignotum pro magnifico est > (pp. 162-3), 
Opere, V, $94-5$ (degn. I); - errori attribuiti dal Vico a Cicerone, Seneca e Grozio (p. 163), V, 321; - la * boria delle nazioni ? (ivi), V, 60, 95; - l'illusione dell'uomo al buio in una stanza piccolissima (p. 164), V, 58; - la « boria dei dotti »(ivi), V, 63, 95-6; - l'errore « borioso " commesso da Platone, specialmente nelle ricerche del Cratilo (ivi), V, 114; - esempî di errori " boriosi " commessi da altri dotti (ivi), V, 196-7; - l'errore "borioso " commesso dallo stesso Vico nel De antiquissima (ivi), IV, 228, 365; - la ridicolezza dell'immaginata " successione delle scuole per le nazioni " e dei sognati viaggi, sopra tutto di Pitagora (ivi), III, 309; IV, 26; V, 63.4, 79; - le principali invenzioni si riscontran simili presso i popoli primitivi, perché occasionate privatamente in ciascuno dalle medesime necessità (p. 165), $\mathrm{V}$, 98.9 (degn. XIII); 一 ̀े inutile supporre un'efficacia del diritto ateniese o di quello mosaico sul romano (ivi), IV, 97; Sciensa nuov seconta, ediz. Nicolini, pp. 1101-3; - la dottrina pitagorica della trasmissione delle anime si ritrova perfino in India (ivi), Opere. IV, 29-30; - i greci fino alla generazione precedente a Tucidide non conoscevano le proprie antichità (ivi), IV, $28 ; \mathrm{V}, 84$; - soltanto ai tempi di Senofonte ebbero notizia delle cose persiane (ivi), IV, 31; V, 85; - Roma fu città nuova fondata tra molti popoli piú antichi (ivi), IV, 27; - Livio non si fa mallevadore della storia romana anteriore alle guerre cartaginesi (pp. 165-6), IV $28 ; \mathrm{V}, 93 ;$ - la storia greco-romana primitiva è, per la sua incertezza, quasi res mullius (p. 167), V, 93; - le lingue sono i testimoni piú gravi degli antichi costumi dei popoli (ivi), V, 100 (degn. XVII); - le etimologie di "intelligere " $\mathrm{e}$ " dissercre " (p. 167), IV, 201; V, 115-6; - la maggior parte delle espressioni intorno a cose inanimate si svelano trasporti dal corpo umano (ivi), V, 182-3; - i tre " etimologici " e il « dizionario di voci mentali " vagheggiati dal Vieo (ivi), IV, 236-7, 237-8, 239-41, 241-3 e cfr. V, 211; - i quattro sensi pei quali, nel Diritto universale, il Vico pensò fossero passati gli dèi (pp. 167-8), III, 451-2; - il significato primitivo del motto "Conosci te stesso "(p. 68), IV, 156, 229; V, 189; - come, quando e perehé furono intrusi significati osceni nei miti (ivi), III, 454; IV, 185; V , 74-5, 113;- esempî varî: 1. Giove, Ginnone, ecc. (ivi), V, 73-4; - 2. Apollo e Dafne 
(pp. 168-9), III, 452; IV, 185; V, 265; - 3. cesto di Venere (p. 169), $\mathrm{V}, 246$; - 4. gli oroi detti " figli di Giove " (ivi), IV, 183-4; V, 245 ; - i " grandi frantumi (o " rottami ») dell'antichità " (p. 163), IV, $244 ; \mathrm{V}, 149$; - la tradizione egiziana delle tre età degli dèi, degli eroi e degli uomini (ivi), IV, 251; V, 60, 105, 198; - la lingua degli dèi ricordata da Omero (ivi), V, 105, 203; - gli elenchi di nomi di dèi attribuiti dal Vico a Varrone (ivi), V, 105; il luco di Romolo (ivi), IV, 99; V, 102; - perché tutti codesti "frantumi " restassero fino al Vico inutili alla scienza (ivi), $\mathrm{V}$, 149 ; - $\mathrm{i}$ " vestigi restati in marmo " (pp. 169-70), IV, $75 ;-$ le carni arrosto e le carni lesse (p. 170), V, 430-1; - il principio dell'eroismo scoperto nella storia romana serve a spiegare o suppliro le storie favolose di altri popoli (ivi), IV, 88-9; - i rapsodi dei poemi omerici e i cantastorie del Molo di Napoli (ivi), $\mathrm{V}, 447$; - le metamorfosi cantate dai pocti antichi e le moderne fiabe dell'orco e delle fate o le favole medievali su Merlino (p. 170-1), III, 273; - il mito del focolare (p. 171), V, 259.60; - i serpenti mitici e il biscione doi Visconti (ivi), IV, 213; V, 270; - i geroglifici e i rébus de Picardie (ivi), V, 202.

XV. - I re primitivi furono immaginati filosofi, per es. da Platone (p. 173), Opere, V, 117, 255; - gli eroi, vagheggiati pronti a sacrificarsi per la comune felicità (ivi), V, 359; - i pretesi viaggi d'istruzione compiuti da uomini di stato per riportare in patria buone leggi (ivi): cfr., per l'esempio delle Dodici Tavole, su cui il Vico insiste a preferenza, III, 476-81; Scienza nuova seconda, ediz. Nicolini, 1061 sgg.; - il fraintendimento delle parole " re ", "libertì ", " popolo " (p. 178), Opere, V, 356, 359; - fece erroneamente credere la monarchia assoluta la forma originaria di governo (ivi), V, 118, 255; - sopra tutti al Bodin (pp. 174-5), V, 356, 515 sgg.; - ma i re di Roma e di Sparta non furon monarchi (p. 175), V, 520-1; - i veri monarchi primitivi furono nelle loro famiglie i singoli eroi (ivi), V, 255; - il carattere aristocratico della rivoluzione contro i Tarquinî e le analogie tra i consoli romani e i re spartani (ivi), V, 357, $520 \mathrm{sgg}$; ; - il " popolo" primitivo fu composto di soli patrizî (ivi), V, 520; - la " libertà " primitiva fu soltanto " signorile" (ivi), V, 357, 520; - la " patria "primitiva soltanto "res patrum" (ivi), III, $399 ; \mathrm{IV}, 94 ; \mathrm{V}$, 
299 ; - nessuna partecipazione ebbe la plebe all'elezione dei re (ivi), $\mathrm{V}, 355-6$; - il giuramento degli eroi greci d'esser nemici eterni della plebe (ivi), V, 121; - la crudeltà del patriziato romano contro la plebe (pp. 175-6), V, 361-3; - gli eroi eran durissini ancho verso le loro famiglio (p. 177), V, 363-5; - i loro giuochi, faticosissimi (ivi), V, 364; - lo loro guerre, atrocissime (ivi), V, 286, $364 ;-$ le schiavitú in cui tenevano i vinti, severissime (ivi), V, $364 ;-$ gli stranieri eran da loro considerati nemici (ivi), V, 320, 342 ; - l'inospitalità delle nazioni primitive (ivi), V, 80 ; - i ladronecci e corseggi eroici (ivi), V, 311; - gl' a imperî paterni ciclopici » (p. 178), V, 363; - l'eta dell'oro fu tutta un " fanatismo di superstizione $\approx$ (ivi), V, 253 ; - le vittine umane offerte agli dèi dai popoli primitivi (ivi), V, 251-3; - l'origine storica del culto religioso (ivi), V, 168; - dei matrimonî (ivi), V, 243; delle sepolture (ivi), V, 262; - i ciclopi primitivi furono, insieme, re, sapienti (in divinazione) e sacerdoti (ivi), III, 302; - le prime sedi degli eroi furon le alture dei monti presso le fontane perenni (ivi), V, 259; - i primi matrimonî, aqua et igni (pp. 178-9), V, 259 ; - il vero concetto che avevan del lecito gli nomini primitivi (p. 179), V, 250; - tardi quei primi aggruppamenti umani scesero alle pianure e poi alle marine (ivi), V, 126; - piú tardi ancora navigarono e dedussero colonie (ivi), IV, $151 \mathrm{sgg.;} \mathrm{V}$, 308; - le gentes maiores e le gentes minores (ivi), V, 132; - le famiglie primitive furon composte, non di soli figliuoli, ma anche di famoli (ivi), V, 277; - ossia di deboli rifugiatisi presso i forti (ivi), V, 279; - o che, coltivando le terre dei padri, furon detti "nexi * (pp. 179-80), V, 282; - e clientes" (p. 180), V, 281; donde il principio eterno dei feudi (ivi), V, 119, 311 sgg.; - provato anche da quel che Tacito (Germania, 14) dice dei famoli dei Germani (ivi), V, 283; - i figli e i famoli, di fronte al pater, si distinguevano soltanto pel none (ivi), V, 281; - i frequenti ammutinamenti dei famoli fecero alleare i patres tra loro, da che sorse la città eroica (ivi), V, 299; - della quale i famoli costituirono la prima plebe senza né diritti politici, né matrimonî solenni, né testamenti (ivi), V, 309-10; -- i quali ultimi avevan carattere politico d'imperio (ivi), III, 344-5; - esclusione dei plebei dai comizî curiati (ivi), V, $307-8,332$; - i re prinitivi furon conduttori 
dei patrizî nella resistenza contro i famoli (ivi), V, 299-300; - i feudi nobili armati e i feudi rustici (p. 181), V, 312; - la vera origine della proprieta (ivi), IV, 83-4; - il dominio bonitario, quiritario ed eminente (ivi), IV, 103; V, 312-3; - perché nelle repubbliche eroiche i patrizì si guardassero dall'arricchire le plebi e fossero rattenutissimi dal venire alle guerre (ivi), V, 121 ; nelle repubbliche eroiche mancavan leggi per punire le offese private (pp. 181-2), V, 122; - donde la necessità dei duelli e delle rappresagiio (p. 182), V, 482; - duelli famosi: Menelao e Paride, Orazî e Curiazî (ivi), V, 484; - posteriormente s'ebbero giudizî per formole verbali scrupolosissimamento interpetrate (ivi), V, 485; come per es. la "lex horrendi carminis" di Roma primitiva (ivi), $\mathrm{V}, 240,485$; - la qual religio verborum, ritenuta anche nel diritto romano degli ultimi tempi della repubblica (ivi), V, 508; - porse argomento a piú commedie di Plauto (ivi), V, 486; - ristrettissimo ora il primitivo diritto privato, che ignorava i contratti consensuali (pp. 182-3), V, 293-4; - il simbolismo dei contratti e delle procedure di tempi piú civili deriva dalla materialità e dalla violenza dei contratti e procedure dei tempi primitivi (p. 183), come, per os.: nelle finzioni della forza nei riti matrimoniali, $\mathrm{V}$, 246; nella mancipazione, nell'usucapione, nelle rivendicazioni, $V$, $526-8$; nelle tante maschere portate in piazza quante eran le persone, V, 528-9; nell'Eredità finta domina delle robe ereditarie, V, 529 ; - l'antica giurisprudenza insomma fu tutta poetica (pp. 183-4), $\mathrm{V}, 530$; - in versi adonî infatti vanno a terminare parecchio leg.ri delle Dodici Tavole (p. 184), V, 22ł; - che i fanciulli romani imparavano a memoria, come quei cretesi le legrgi di Minosse (ivi), V, 225; - poetiche del pari le leggi degli egizî, di Licurgo, di Dracone (ivi), V, 225; - il diritto romano antico fu un " poema se. rioso " (ivi), III, 347; IV, 225; V, 531; - la poesia ebraica, araba, egizia (ivi), V, 224; - in verso consegnarono le loro prime storie $\mathrm{i}$ persiani, i chinesi, i germani e gli americani (pp. 18t-5), V, 225 ; - il verso eroic's (prima spondaico, poi dattilico) e il verso giambico (p. 185), V, 114-5, 222-3; - i numeri poetici eran frequentissimi noi primi prosatori (ivi), V, 219 ; - in giambi fu verseggiata la tragedia o poi, per imitazione, la commedia (ivi), V, 460; quale fosse il « corpo » dei linguaggi primitivi (ivi), IV, 225; - 
gli episodì e il parlare contorto (p. 186), IV, 225-6; V, 219; perché la lingua greca e la francese sian ricche di dittonghi (p. 180), V, 101, 220-1; - la lingua tedesca e le scoperte maravigliose a cui darebbe luago lo studiarne le origini (ivi), IV, 226; $\mathrm{V}, 226-7$; - l'esigenza d'una teogonia naturale e la genesi dei dodici dèi maggiori (ivi), IV, 255-70; V, 175; - Giove o il Cielo (ivi), V, 227 sgg.; - Giunone (pp. 186-7), V, 246 sgg.; - Diana (p. 187), V, 261 sgg.; - Apollo (ivi), V, 265 sgg.; - Vulcano, Saturno, Cibele (ivi), V, $274 \mathrm{sgg}$.; - Marte (ivi), V, $286 \mathrm{sgg.;}$ Venere (ivi), V, $289 \mathrm{sgg}$; - Venere celeste e Venere plebea (ivi), V, 291 sggr; - il duplice significato anche dei miti di Vulcano e di Marte (p. 181), V, 295 sgg.; - Tantalo, Issione, Sisifo (ivi), V, 298-9; - Ercole che lotta con Anteo (ivi), V, 32t-5; - Minerra (ivi), V, 304 sgg.; - Mereurio (ivi), V, 316 sgg.; - Nettuno (ivi), V, 339 sgg.; - Minosse (ivi), V, 338; - gli argonauti, la guerra troiana, il ritorno di Ulisse, Europa e il toro, il Minotauro (ivi), V, 339; - Perseo, Teseo (ivi), V, 340; - Zoroastro (ivi), V, 61-2, 63 ; - i due Mercurì Trismegisti (ivi), V, 67 sgg., 71; - Orfeo (ivi), V, 72 sgg.; - Confucio (ivi), V, 58, 194; - Esopo (pp. 187-8), V, 192-3; - Dracone (p. 188), V, 191-2; - Solone (ivi), V, 188 sgg.; - i sette re di Roma (ivi), V, 190-1; - il decemvirato e le Dodici Tavole (ivi), V, 191; Sciensa nuova seconda, ediz. Nicolini, pp. 1093-1141; - Platone introdusse nel mito di Giove l'idea dell'etere (ivi), Opere, IV, 192; V, 249; -- altri filosofi in Minerva, che esce dal capo di Giove, veggon descritta la sapienza divina (ivi), V, 304; -- altri ancora, nel caos e nell'orco', la confusione dei semi universali della natura (ivi), IV, 191; - cosa fossero invece per gli uomini primitivi il caos e l'orco (p. 189), V, 369; a che cosa corrispondessero per essi i quattro elementi del mondo (ivi), V, 371; -- como essi concepissero l'essere e il sussistere, la compagine del corpo e tutte le funzioni interne dell'anima (ivi), V, 372 sgg.; - come il cielo astronomico (p. 183), V, 383; - come l'inferno (ivi), V, 386; - come la terra (ivi), V, 389; - l'astronomia poetica (ivi), V, $391 \mathrm{sgg}$; - la eronologia poetica (pp. 18990 ), 'T, 394 sgg.; - la greografia poetica (p. 190), V, 403 sgg.

$\mathrm{XVI}$. - A Omero non poté appartenere quella sapienza riposta che comunemente gli si suole attribuire (p. 191), V, 422; - 
quale stravagante filosofo (ivi), V, 425 ; - e quale stolto ordinatore della civiltà greca in tal caso egli sarebbe statol (ivi), V, 424; - quali sconcezze egli fa dire e compiere a Giove, Minerva, Marte, Giunone! (ivi), V, 423-4; - quali eontumelie si scambiano Achille e Agamennone! (ivi), V, 4.24; - gli eroi omerici abbandonano i cadaveri dei nemici a cani e corvi (ivi), V, 423; - si ubbriacano (pp. 191-2), V, 425; - si dimostrano di cortissimo intendimento, come oggi i contadini (p. 192), V, 380-1; - per un nonnulla passan dal rise al pianto, dalla calma alla-collera bestiale (ivi), V, 426; - son tutti passione e irriflessione (ivi), V, 381 ; - carattere di Achille (pp. 192-4), V, 359.61, 381, 360, 426, 360-1; - le comparazioni omeriche hanno per oggetto belve o altre cose selvagge (p. 194), V, 425; - i costumi degii eroi omerici o le a favole da vecchiarella $>$ dell'Odissea (ivi), V, 427; lo stile trueulento d'Omero (ivi), V, 425 ; - nei poemi omerici vi sono incoerenze di costumi (p. 195), V, 429-32, 452; - incoerenze di allusioni geografiche (ivi), V, 428; - giacché l' Iliade trabalza all'oriente della Grecia, l'Odissea all'occidente (ivi), V, 448; - incoerenze di linguaggio (ivi), V, 448; - come sorse la strana opinione (alla quale, fino al 1725, aveva acceduto lo stesso Vico: efr. III, 279; IV, 198; VI, 38) che Omero fosse andato raceogliendo il suo linguaggio dalle varie popolazioni greche (ivi), V, 429 ; VI, 42 ; - nessuna fede meritano le vite di Omero dello pseudo Erodoto e dello pseudo Plutarco (ivi), V, 449; - del maggior lume di Grecia s'ignora la patria e il tempo in cui visse (pp. 195-6), V, 427 sgg., 448; - lo si afforma, ciò non ostante, povero e cieco (p. 196), V, 449; - e si dice perfino che da giorane componesse l'Iliade, da vecehio l'Odissea (ivi), V, 448; - ma la serittura non esisteva ai tempi di Omero (ivi), V, 445-148; - le tre iscrizioni eroiche allegate dal Voss sono imposture (ivi), V, 445; - per tutte queste ragioni il Vico sospettò che Omero fosse "per la metà s un carattere poetico (ivi), V, 449.50; - se infatti si pensa che i poemi omerici sieno due grandi tesori dei costumi della Grecia antichissima (ivi), V, 455; - se a un popolo intero poe-tante (ivi), V, 451; - si spiegano le stravaganze delle favole omeriche (ivi), IV, $185 ; \mathrm{V}, 113,43 \mathrm{i} ;$ - si spiegano le varietà dei costumi, l'Omero "giovane * e l'Omero " vecchio ", il vario luogo 
di nascita assegnato a Omero, la varietà dei dialetti, ecc. (pp. 196-7), $\mathrm{V}, 451-2$; - Omero pertanto deve essere sperduto dentro la folla dei poeti greci (p. 197), V, 452; - e considerato come un carattere poetico di uomini greci in quanto narravan cantando le loro storie (ivi), V, 450; - cosi le sue pretese sconcezze e inverisimiglianze diventano acconcezze e necessità (ivi), V, 450 ; - egli è stato il primo storico della gentilita (ivi), V, 40̃4; - in lui si ha il documento della primitiva identità di storia e poesia (ivi), V, 444; - e la conferma che prima d'Ecateo Milesio la storia dei greci fu scritta da poeti (ivi), V, 445; - nell'Odissere di chi ha ben raccontata una storia si dice averla narrata da aedo (ivi), V, 445 ; - il Vico propende per due principali autori-poeti (ivi), V, 448,452 ; - « omero » significa per lui « compositore e legatoro di favole 》 (ivi), V, 445; - quel che egli dice dei rapsodi e della redazione pisistratea (p. 198), V, 445-6; - "nisi ita scepe dorinituret, munquam bonus fuisset Homerus 》 (p. 199), III, 235; appunto perché non filosofo, Omero fu poeta grandissimo (p. 200), $V, 443,453$; - ebbe memoria vigorosa, fantasia robusta, ingegno sublime (ivi), V, 453; - i caratteri eroici si seppero fingere soltanto da lui (ivi), V, 435, 442; - le sue comparazioni sono incon larabili; le sue sentenze, sublimi (ivi), V, 442; - la sua locuzione è piena d'evidenza e di splendore (ivi), V, 453; - la sua favella è tutta per somiglianze, inmagini, paragoni (ivi), $\mathrm{V}, 442$; non delicato ma grande (ivi), V, 4t1; - egli è il padre e principe di tutti gli altri poeti (ivi), V, 434,453; - i poeti ciclici $\theta$ i cantastorio del Molo di Napoli (pp. 200-1), V, 447; - congetture del Vico sull'età di Esiodo (p. 201), V, 81, 446-7; - e di Esopo (ivi), $\mathrm{V}, 78$; - le tre epoche della lirica greca (ivi), V, 457-8; - la tragedia greca (p. 195), V, 434,456-60; - la commedia antica e nuova (pp. 201-2), ivi; - il precetto d'Aristotele sugli eroi di tragedia e l'eroismo \& galante $>$ foggiato dai poeti di tempi tardi (p. 202), V, 380-1; - il « pastoreccio galante " di Mosco e Anaerennto (ivi), IV, 178; - perché nella letteratura greca e romana furon serbati con tanto rigore i confini tra la poesia e la prosa (ivi), VI, 46; - i poeti salî della primitiva letteratura latina (ivi), V, 456 ; - $\mathrm{i}$ * frantumi degli inni saliari (p. 203), V, 224; - Livio Andronico, Novio, Ennio (ivi), V, 226; - i romani perderon di 
veduta la loro storia degli dèi e conservarono in favella volgare soltanto la loro storia eroica (ivi), ${ }^{\circ} \mathrm{V}, 100-1$; - Virgilio (ivi), V, 388; IV, 200; - Lucrezio (ivi), VI, 138; - Orazio (pp. 203-4), $\mathrm{V}, 458$; - la poesia fu la lingua primitiva anche della nazione ebraica (p. 204), III, 294; IV, 163; - parallelo tra Mosè e Omero, e il detto "Ego sum qui sum" (ivi), IV, 25; - carattere del decalogo (p. 198), IV, 145; V, 131; - il detto di Teofrasto sugli ebrei * fllosofi per natura 》 (ivi), III, 265; IV, 187; V, 131.

XVII. - Nel De antiquissima il Vico aveva dato alla storia di Roma lo sfondo di un'antichissima cultura italica proveniente dall'Egitto (pp. 207-8), II, 59-60, 142-3; - in séguito ritenne ancora Pitagora cultore d'italica sapienza (p. 208), II, 114 ; III, 309; ma poi abbandonò definitivamente la tesi della derivazione degli istituti romani da istituti stranieri, professando d'avere errato sull'esempio del Cratilo platonico (ivi), IV, 228; - per lui con la storia di Roma non comincia il mondo (ivi), IV, 27 ; - suo punto di partenza è l'asilo di Romolo (ivi), IV, 249-50; V, 86-7, 102, o prassim; - niente colonia troiana (ivi), V, 416; - la leggenda della renuta di Enea in Italia è il frutto dell'incrocio della boria greca e della boria romana (pp. 208-9), V, 416; - preferibile l' ipotesi che $\mathrm{i}$ romani in tempi antichissimi avessero distrutto una colonia greca nel lido del Lazio (p. 209), V, 410-1; - il periodo regio a Roma fu periodo non di monarchia ma di aristocrazia (ivi), V, 355-8; - i re di Roma furon simboli di istitnzioni ginridiche (ivi), V, 190-1; - il censo di Servio Tullio fu pianta di libertà non popolare, nua signorile (ivi), V, 325-8; - la rivoluzione contro i Tarquinî obbe carattere aristocratico, non popolare ( $\mathrm{pp}$. 209-10), V, 355-8; - la * clatis historice romance universce » (p. 210), III, 473; - è vaga la spiegazione che della grandezza romana adduce Polibio (ivi), III, 474; V, 510; - sbaglia anche il Machiavelli, cosí quando la riferisce a istituti sparsi (ivi), III, 474; come quando ne arreca quale causa la magnanimità della plebe (ivi), V, 510; - inferiore a tutti è Plutarco, che la ripone nella fortuna (ivi), III, $474 ; \mathrm{V}, 510$; - i romani ebbero giovane l'eroismo (ivi), V, 102; - i patrizî furono forti nel custodire il loro ordine e la religione su cui si fondava (ivi), V, 122; - giacché, quando i nobili disprezzano una religione nativa, è gran segno 
che una nazione vada a perdersi (ivi), $\mathrm{V}, 275$; - la plebe fu magnanima nel volere religione, auspicî e diritti civili, e i giureconsulti sapienti nell' interpetrar le antiche leggi secondo le mutate condizioni sociali (ivi), V, 122; - l'impero romano s'ingrandi e durò perché restò sempre fermo sui suoi principî (pp. 210-1), V, 509 ; - le gare tra patrizî e plebei nelle guerre (p. 211), V, 122 ; quattro norme applicarono i romani cosí nelle lotte interne tra patrizî e plebei, come nella conquista del mondo (ivi), IV, 148.51; V, 344; - come la plebe ottenesse il dominio bonitario, poi il quiritario e con questo la legge delle Dodici Tavole (ivi), IV, 110-2; V, 86-7; Scieña nuova seconda, ediz. Nicolini, pp. 1133-7; - con questa la plebe conquistò il diritto scritto e cessò l'areano onde i patrizî avvolgevano le leggi (ivi), Opere, V, 123; - i patrizî accolsero i desiderî della plebe non con la condiscendenza che dice Livio, ma con la pitrosia che narra Dionigi d'Alicarnasso (ivi), V, 123; - la leggrenda dei tre commissarî inviati ad Atene per riportare a Roma quella che fu poi la legge delle Dodici Tavole venne creduta da Livio e Dionigi (p. 212), Sc. nuo. sec., ed. cit., pp. 1119-22; - ma ignorata da Polibio (ivi), Opere, VI, 146-7; V, 124-5; - e nou creduta da Cicerone (ivi), III, 481; V, 126-7; Sc. nuo. sec., ed. cit., pp. 1125-7; - come mai all'ancor barbara Roma sarebbe potuta giungere la fama di Solone? (ivi), Opere, IV, 66 ; - come i romani avrebbero potuto aver notizia cosi esatta circa la qualità delle leggi ateniesi? (ivi), IV, 67; - erall possibili allora anbascerie tra greci o romani? (ivi), IV, 67; Sc. nuo. sec., ed. cit., p. 1095 ; - come mai si trovava proprio a Roma Ermodoro per tradurre le leggi portate di Grecia? (ivi), Sc.nuo.sec., ed. cit., pp. 1095, 1097; - come egli avrebbe fatto a tradurle con tanta latina purità? (ivi), Opere, IV, 67; Sc. nuo. sec., ed. cit., p. 1125; - che dire della falsissima lettera di Eraclito a Ermodoro? (ivi), Opere, IV, 67; Sc. nuo. sec., ed. cit., pp. 1095-6; impostura siffatta lettera, impostura la venuta d'Ermodoro in Italia, impostura la sua statua nel Comizio (pp. 212-3), Opere, IV, 67-8; Sc. nuo. sec., ed. cit., pp. 1097-1110; - l'origine ateniese delle Dodici Tavole è una boria di dotti, ehe le fecero provenire via via da diversi popoli (p. 213); Sc. nuo. sec., ed. cit., pp. 1122-1140; le siniglianze, che esse presentano con le leggi ateniesi, spartane 
e di altri popoli, son da attribuire al corso uniforme delle nazioni (ivi), Opere, V, 69; $\$$ c. nuo. sec., ed. cit., pp. 1101-5; - come si spieghi la disposizione decemvirale sul lusso dei funerali (ivi), Opere, V, 191; Sc. nuo. sec., ed. cit., 1106-8, 1140; - la legislazione decemvirale divenne un carattere poetico (ivi), Opere, IV, $70 ; \mathrm{V}, 191 ;$ s $\%$. moo. sec., ed. cit., p. 1140; - ma la legge originaria delle Dodici Tavole, rozza, inumana, crudele e fiera (ivi), ivi, pp. 1117-8; Opere, V, 85; - è un gran testimonio dell'antico diritto naturale delle genti del Lazio (ivi), V, 100; - la lotta pei connubî e la rogazione canulea (pp. 213-4), IV, 54-5, 123; V, 85, $310,495-6$; - quando fosse effettivamente promulgato il cosí detto censo di Servio Tullio (p. 214), V. 33-4, 325-8; - le successire concessioni fatte alla plebe e la Lex Publilia (pp. 214-5), V, 89-91; la Lex: Petelia (p. 215), V, 91; - la riforma di Fabio Massimo (ivi), V, 331; - il dominio sorrano del senato e il senatoconsulto ultimce necessitatis (pp. 215-6), V, 91-2; - i Gracchi e le guerre civili (p. 216), V, 125; - come dalla famiglia romana dell'epoca aristocratica (ivi), V, 499, 501; - si giungesse a quella dell'epoca democratica (ivi), $\mathrm{Y}, 502$; - analoghi mutamenti accaduti negli istituti della proprietà (pp. 216-7), V, 327-8, 493-4; - nelle procedure giudiziarie (p. 217), V, 531-2; - nelle " culessce" (ivi), V, 534 ; - dal certo delle leggi si giunse all'cequum bonum (ivi), $\mathrm{V}$, 134, 534-5; - da pene sererissime a pene miti (ivi), V, 521-2; - le leggi si moltiplicarono (pp. 217-8), V, 507-8; - men crudeli divennero le guerre (p. 218), V, 523; - tendenza delle democrazie e delle monarchie alle conquiste (ivi), V, 524 ; - negli Stati aristocratici, per altro, al contrario degli Stati popolari, l'iuteresse pubblico è preposto alle utilità private (ivi), V, 475-6; - l'origine spontanea della monarchia (pp. 318-9), V, 62, 401; - la favoleggiata : legge regia " (pp. 219-20), V, 125-6, 513-5; Scienza nuога seconda, ediz. Nicolini, pp. 1145-53; - sotto l'impero si sciolsero sempre piú i rigidi vincoli della famiglia gentilizia, mercé l'incremento dei fedecommessi e dei testamenti (p. 220), Opere, $\mathrm{V}, 503$; l'istituzione dei tre peculî (pp. 220-1), V, 503-4; - l'indebolimento della patria potestà (p. 221), V, 50ł; - l'alleviamento della schiaritú (ivi), V, 505; - le pene si addolcirono ancora (p. 213), V, 52.-3; - nell'interpetrazione delle leggi prese il sopravrento 
l'equità naturale (ivi), V, 508; - la cittadinanza fu concessa da Caracalla a tutto il mondo romano (ivi), V, 523; - dai singoli editti dei pretori si passò all'Editto perpetuo (p. 222), V, 523; il diritto naturale delle genti, insomma, cede il posto al diritto naturale delle nazioni (ivi), V, 505; - e si ritorna, dopo lungo svolgimento, a quell'uno ch'era delle monarchie familiari dei primi padri (ivi), V, 524-5 ; - possibile è ormai soltanto imbarbarirsi con la "barbarie della riflessione rinità, per ripassare a una nuova ed eroica barbarie (ivi), V, $570-1$.

XVIII. - La barbarie seconda o ricorsa riuscí al Vico piú oscura della barbarie prima (p. 224), V, 33, 536-7, 550; - il nessun risalto dato dal Vico al cristianesimo (p. 225), V, 537; - risorgono gruppi di case sui monti (pp. 225-6), V, 259; - risorgono, con lo chieso e i conrenti, gli asili (p. 226), V, 5.t0; - risorgono i feudi (ivi), V, 556 ; - la fendalità medievale è un diritto antichissimo rinnovellato con l'ultima barbarie (ivi), IV, 101; non vile ma eroica ̀̀ la materia dei feudi (pp. 226-7), V, 555.6; ritorna la divisione tra eroi e famoli (p. 227), V, 542, 54t-5; il feudo ò offottivamente, come vien tradotto dai feudisti, « clientela $\gg$ (ivi), V, 543; - le clientole di Romolo o i feudi personali ded medioevo (ivi), V, 545̆; - gli kmet polacchi (ivi), V, 546; i feudi rustici medievali (ivi), V, 546-7; - che i feudisti tradncono eccellentemente * beneficia (ivi), V, 545; - i « nexi » dei tempi primi e gli « nomini ligi » dei tempi secondi (ivi), V, $547,-1$ ' opera militaris " romana $\mathrm{o}$ il * militare servitium " medievale (ivi), V, 5ł2; - i * socî * di Roma antica e i feudi soggetti a maggiore sovranità medievali (pp. 227-8), V, 547; - gli Stati aristocratici medievali e dei tempi moderni: Svezia, Danimarca, Polonia (p. 228), V, 555; - il senato di Romolo o i parlamenti medievali (ivi), V, 552-3; - le cause discettate in questi (ivi), V, 554; - il Sacro Real Consiglio napoletano (ivi), V, 554-5; carattere religioso dei feudatarî (ivi), V, 540; - $\theta$ dei re medievali (ivi), V, 537; - Ugo Capeto (pp. 228-9), V, 537; - le * religioni armate " del medioevo e $\mathrm{i}$ * pura et pia bella * ricorsi (p. 229), V, 537-8; - le schiavitú eroiche ricorse (ivi), V, 539-40; - la caccia alle reliquie di santi e l'obbligo nei popoli vinti di riscat- 
tar le campane (ivi), V, 538; - i beni allodiali e i beni feudali (pp. 229-30), V, 551-2, 327-8; - le cnfiteusi, i censi, le precarie, i livelli medievali (p. 230), V, 548; - l'esclusione delle donne dalle successioni e la legge salica (ivi), V, 498, 552; - la crudeltà delle pene medievali (pp. 230-1), V, 522; - ma nemmeno nel medioevo c'erano leggi e procedure pei torti privati (p. 231), $\mathrm{V}, 553$; - le purgazioni canoniche $\gg$ e le rappresaglie (ivi), $\mathrm{V}$, 483,539 ; - i duelli tra i baroni per questioni allodiali, specie nel Regno di Napoli (ivi), V, 550; -- i ladronecci e i corseggi eroici ricorsi (ivi), V, 539; - nel medioevo fu incostantissima la fortuna dei regni (ivi), V, 520; - l'oblio nell'alto medioevo del diritto giustinianeo (ivi), V, 508-9; - tutto a base di consuetudini fu invece il diritto feudale (ivi), III, 165 ; - $\mathrm{i}$ « patti nudi " $\mathrm{e}$ i \& patti vestiti » (ivi), V, 549; - l'aneddoto leggendario su Corrado III di Hohensthaufen e la presa di Weinsberg (pp. 231-2), V, 488; ritornarono i tempi mutoli o analfabetici (p. 232), V, 231-2; - le lingue italiana, francese, spagnuola e tedesca non eran messe in iscritto (ivi), V, 539 ; - soltanto da pochi ecclesiastici si scriveva un barbaro latino (ivi), VI, 37; - « chierico" perciò equivaleva a * letterato"(ivi), V, 231 ; - tuttavia molti vescovi non sapevan firmare se non col segno di croce (ivi), V, 231; - origine della norma "excellens in arte non debet mori 》 (ivi), V, 234; - perché * letterato » significhi anche * erudito » (ivi), $\mathrm{r}, 234 ;-\mathrm{l}$ 'importanza nel medioevo delle impresc gentilizie (ivi), V, j39; - la prosa dei padri della Chiesa è piena di numeri poetici (ivi), V, 222 ; - perché non si ebbero poemi religiosi cristiani (ivi), VI, 45-6; - i romanzieri medievali credettero di narrare storie rere (p. 233), V, 438-9; - la storia di Turpino sopravvisse in Francia quasi poema omerico (p. 225), V, 101; - Guglielmo Pugliese e Guntero (ivi), V, 226, 438; - poeti furono i primi scrittori volgari in Italia, Francia, Provenza (ivi), VI, 37 ; - nei cavalieri erranti ricorse la virtú puntigliosa degli eroi omerici (ivi), $\mathrm{V}, 361$; Cola di Rienzo (ivi), V, 425 ; - quel che parevano al Vico fanciullo i * molli clivi * (ivi), III, 270; - i paladini medievali eran finti di enorme statura, allo stesso modo che di eccedente grandezza si dipingevan le imınagini degli esseri celesti (ivi), V, 438; - il paragone tra gl' ingegni umani e i terreni incolti (pp. 233-4), VI, 
43; - Daute, Petrarca, Boccaccio (p. 234), ivi ; V, 425; - perché Dante chiamasse il suo poema " commedia » (ivi), V, 439 ; - parallelo tra l'Inferno e l'Ilinde, il Purgatorio e Paradiso e l'Odissea (ivi), VI, 38; - il linguaggio di Omero e il linguaggio di Dante (ivi), III, 279; IV, 198-9; VI, 38, 42; V, 429; - la « divinità " di Dante (p. 235), VI, 39-40; - se Dante non aresse saputo di scolastica e di latino, sarebbe riuscito maggior poeta (ivi), IV, 200; in qual guisa si debba commentare la Dirina Commedia (ivi), VI, 44; - il teatro inglese (ivi), VI, 38; - i poeti slesiani (p. 236), $\mathrm{V}, 226$; - la polemica del Vico contro il Bodin a proposito dell'origine della monarchia francese (ivi), V, 5\%6; - il mutamento dell'ossequio al barone nell'obsequium principis, V, 544; - il risorgere del diritto giustinianeo e la civiltà dei tempi moderni (pp. 236-7), V, 557-8.

XIX. - Per questo capitolo si vedano il De studiorum ratione, le prime pagine dell'autobiografia e le lettere all'Esperti (Opere, VI, 3-6), al De Vitry (VI, 7-10) e al Solla (VI, 10-7). Per l'accenno alla sapienza, Opere. $\mathrm{V}, 153$. 


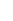


APPENDICE 
$$
\text { . }
$$ 
Intoryo alla vita e al Carattere di G. B. Vico *

Alla trasfigurazione rettorico-leggendaria, che negli inizî e nel ferrore del Risorgimento nazionale, si compi dei poeti, dei filosofi, di quasi tutti gli uomini piủ o meno rappresentativi della storia italiana, atteggiandoli come patrioti, liberali, ribelli, o almeno frementi, contro il trono e l'altare, si tentò per un momento di sottomettere anche, cou lieve toceo di bacchetta magica, Giambattista Vico. E si disse, tra l'altro, che il Vico, consapevole della grave scossa che il suo pensiero dava alle tradizionali credenze religiose, e messo in guardia da amici, si era industriato a eingere di

* Poiché il lavoro precedente è rigorosamente ristretto all' analisi della filosofia víchiana e non dì alcun cenno della vita e del carattere personale del Vico, non riuscirà sgradito trovare in appendice la conferenza, che su quest' ultimo argomento io tenui alla Società napoletana di storia patria il 14 aprile 1909 , e che, messa in iscritto, fu poi inserita nella Voce di Firenze (a. I, n. 43, 7 ottobre 1909). - Aggiungo, per memoria, che il Vico nacque in Napoli il 23 giugno 1668 (non 1670, com'egli dice nell'autobiografia), e morí il 23 genuaio 1744 (non il 20, come dicono tutti i biografi): cfr. nella nuova edizione dell'Autobiografia, carteggio e poesie varie (Bari, Laterza, 1911), pp. 101, 123, 124. 
tenebre la Scienza nuova, in modo che solo i fini intenditori potessero scorgere dove andasse a parare. Ma questa leggenda, che corse soprattutto tra i patrioti napoletani dei primi dell'Ottocento, non poté reggere a lungo, nonché alla critica, al lume del buon senso; c Cataldo Iannelli, fin dal 1817, ne fece giustizia ${ }^{\text {. }}$

E certo, nel riguardo oggettivo, che le dottriue del Vico recavano implicita una critica cosi della trascendenza cristiana e della teologia come della storia del cristianesimo. Potrà darsi, nel riguardo soggettivo, ehe il Vico, durante la sua giovinezza (della quale sappiamo ben poeo), fosse travagliato da dubbî religiosi. Oltre che nelle sue letture, egli poteva trovare tentazioni a questi dubbî nellat societì dei giovani suoi coetanci, tra i quali non crano rari $\mathrm{i}$ \& libertini », o, come anche si trovano chiamati nelle scritture di allora, gli « epicurei " e « ateisti ${ }^{2}$. In una lettera del 1720 al padre Giacchi, egli dice che in Napoli si ricordavano di lui " fin dalla sua prima giovinezza e debolezze ed errori », e questi, fissi nella memoria, diventavano, come accade, * criterî eterni per giudicare tutto il bello e compito che per avventura altri faccia di poi ${ }^{3}$. Quali

1 Si veda, per tutta la questione, Croce, Bibliografia vichiana, pp. 91-5.

2 Nei Giornali del Confuorto (mss. nella Bibl. della Società stor. napol., XX. C. 22, vol. III, f. 111), sotto l'agosto 1692: * Sono state carcerate nelle carceri di San Domenico del tribunale del Sant'Officio alcune persone civili, tra quali il dottor Giacinto di Cristofaro, figlio del dottor Bernardo; e molti altri sono scampati via, quali seguitano la setta degli epicurei o ateisti, volendo che l'anima morisse col corpo . . Il De Cristofaro è il noto matematico e giureconsulto napoletano, pel quale si veda F. Axodeo, Vita matematica napoletana, parte III (Napoli, Giannini, 1905), pp. 31-44, e fu amico del Vico. Altre notizie intorno agli a epicurei > di Napoli di quel tempo, in Carducci, Opere, vol. II, pp. 235̄-6.

3 Lettera del 12 ottobre 1720. 
erano mai codesti errori e debolezze? - E quando useí il De universi iuris uno principio et fine uno, anzi la Sinopsi che ne dava il programma, « le prime voci » avverse, che il Vico sentí levarsi, "erano tinte da una simulata pietà » contro le quali egli trovò scudo e conforto nella religione stessa, cioè nell'assenso del Giacchi, « primo lume del piú severo e piú santo ordine de' religiosi »'. Ma come delle accuse che su questo punto gli si facevano non ci resta notizia particolare, cosi dei dubbî religiosi, che poterono travagliarlo, non si ha nemmeno la generica certezza. Tutti gli scritti del Vico mostrano che nel suo animo si assidera grave, salda, immota, come colonna adamantina, la religione cattolica: salda e forte cosí da non essere neppure in piccola parte intaccata dalla critica, che egli inaugrurava, dei miti. Né soltanto in tutte le esteriori dimostrazioni il Vico fu cattolico irrepronsibile, e sottomise sempre ogui parola che mettesse in istampa alla doppia censura, pubblica e privata, degli amici ecclesiastici, e fra zimarre sacerdotali e cocolle fiatesche, piú ancola che fra toghe di giuristi, menò la sua vita filosofica e letteraria; - ma egli giunse perfino allo scrupolo d'intermettere il commento al Grozio, non sembrandogli dicevole che un cattolico commentasse un antore protestante ${ }^{2}$; ed ebbe cosí delicato punto d'onore cattolico da non accettare nemmeno la polemica circa i suoi sentimenti religiosi: "Questa difficoltit (diceva ai critici del Giomale de' letterati), come quella che mi fate sull'immortalità dell'anima, dove par che premiate la mano con ben sette argomenti, se non mi fusser fatte da voi, io giudicherei che andassero piu altamente a penetrare in parte la quale, quantunque si pro-

1 Ivi.

Autob., ed. cit., p. 39. 
tegga e sostenga con la vita e coi costumi, pure s'offende con la stessa difesil. Ma trattiamo le cose ! ${ }^{1}$. Il suo cattolicesimo si mostra scevro di materialità e superstizioni, cosí generali nel costume del tempo, e specie a Napoli dove in ogni avvenimento della vita privata e pubblica interveniva attore e direttore san Gennaro: era cattolicesimo di animo e di mente alta, e non di volgo. Ma neppure contro le credenze popolari e le superstizioni il Vico assunse le parti di censore; - pago di non parlarne, come non si parla delle debolezze di persone e d'istituzioni che sono oggetto della nostra reverenza.

\section{II}

Disposizione d'animo analoga per piú rispetti a quella verso la religione ebbe il Vico verso la vita politica e sociale. Non era nulla in lui dello spirito combattivo da apostolo, propagandista, agitatore e congiurato, che fu di alcuni filosofi della Rinascenza; in ispecie di quel Brano e di quel Campanella, che egli (benché, e forse perché, napoletano) non nomina mai. Certo, il suo tempo e il suo paese non furono luogo e tempo di rivolgimenti e rivoluzioni e di quegli ardenti contrasti che suscitano grandi azioni e passioni politiche. Pure, vi si agitarono partiti politici (il francese e l'austriaco), e si profilỏ un certo desiderio d'indipendenza nazionale, e sorsero womini che dettero l'opera e la vita a questi fini, e furono perseguitati $\mathrm{c}$ andarono profughi; e, segnatamente, giungeva in quel tempo al piú alto punto la lotta dello Stato contro la

1 Le cose, cioé, non le obiezioni religiose, che a lui suonavano come offesa personale (Rivposta al Giornale de' leiterati, in Orazioni ecc., ed. Gentile-Nicolini, pp. 266-7). 
Chiesa, e di Napoli contro Roma, con Pietro Giannone, del quale come di tutto quel movimento il Vico tacque sempre e parre non essersi nemmeno accorto. La vita politica stava alta sopra il suo capo, come il cielo e le stelle; ed egli non si protese mai nel vano sforzo di attingerla. Come le controversie religiose, cosí quelle politiche o sociali furono il limite della sua attivita. Era veramente nomo apolitico. Di che non si può fargli colpa né accagionarlo di fiacchezan, perché ogni uomo ha il suo limite, e una lotta esclude l'altra, un lavoro esclude gli altri lavori.

Non che egli si ritraesse da ogni contatto con la politica e coi rappresentanti di essa. Purtroppo, dovette cor- teggiare assai di frequente e l'una e gli altri, con istorie, orazioni, versi ed epigrafi, latini e italiani; i quali basterebbero da soli a ricostruire la serie delle vicende cui ando soggetta Napoli dalla fine del secolo decimosettimo alla metà del decimottavo: il viceregno spagnuolo, la congiura e rivoluzione tentata dagli autonomisti, la reazione e il rassodato viceregno spagnuolo, la conquista austriaca, il viceregno austriaco, la riconquista spagnuola e il regno di Carlo Borbone... Ma egli, « molto pei suoi bisogni conversevole " ${ }^{2}$, e professore di eloquenza nella regia universita, dovera fornire i componimenti letterarî, richiesti dalle solennita del giorno; eosi come il drappiere lavorava, per le medesime occasioni, le frange, e lo stuccatore le volute e gli srolazzi. E quali frange e quali svolazzi! Perdurava la moda letteraria secentesco-spagnuola; - cio basta per gran parte a spiegare quel che nelle lodi profuse dal Vico ci sembra, ed è, iperbolico e barocco. Del suo animo indifferente e innocente può dare esempio quel

I In Autob., ecc., ed. cit., p. 142. 
luogo dell'autobiografia, dove, dopo aver fatto ricordo del Panegyricus Philippo V inscriptus, da lui composto per ordine dell'ultimo viccré spagnuolo duca di Ascalona, continua, come se niente fosse, col riattaceo di un semplice * appresso ": "Appresso, ricevutosi questo reame al dominio austriaco, dal signor conte Wirrigo di Daun, allora governatore delle armi cesaree in questo regno, ebbe l'ordine di comporre le iscrizioni pei fúnerali espiatorî di Ginseppe Capece e Carlo di Sangro ${ }^{1}$; cioè dei due ribelli contro Filippo $\mathrm{V}$, che il governo precedente aveva messi a morte, qualche anno prima, nella repressione della congiura di Macchia, dal Vico narrata, veridicamente bensí ma con ossequio al governo costituito, nel De parthenopea coniuratione.

Ma non e'è, nel Vico, bassezza; e, se deve dirsi, in quei suoi scritti, retore e panegirista, non può dirsi adulatore. L'adulatore, l'uomo senza coscienza, vilipende e calunnia gli avversarî degli uomini da lui adulati, o colpisce i vinti; e questo è bassezza. Il Vico, il quale, pur conoscendo chi fosse l'italiano, anzi il napoletano, che aveva inviato agli Acta lipsiensia la noterella contumeliosa contro di lui, e fremendo d'ira, e potendo facilmente rovinarlo (perché quella noterella era anticattolica), gencrosamente non volle mai svelare quel nome ${ }^{2}$, presta, si, i suoi servigi di professore d'eloquenza, ma non traffica con gli interessi dei suoi lodati padroni. Della Vita di Antonio Carafa, composta per commissione, e col provento della quale maritò una figliuola, dice che la lavorò * temprata di onore del subietto, di riverenza verso i principi e di giustizia che si dee aver per la verità ${ }^{3}$. E, per tornare

1 Autob., ed. cit., p. 56.

2 Lettera del 4 dicembre 17:29: in Autob., ecc., ed. cit., pp. 209-10. 3 Autob., ed. cit., p. 38. 
al caso sopraricordato del Capece e del Sangro, quando nel De parthenopea coniuratione egli narra la morte di yuei due nemici della parte trionfante, mostra anche allora, in taluni particolari, il suo animo gentile; e del Capece, che non volle arrendersi ai soldati spagnuoli, scrive "ostentans pectus neci eamque infestis armis efflagitans, inexoratus occubuit, fortissimum mortis genus si causa cohonestasset $\bowtie$; e pel Sangro, riferita la voce della grazia fattagli da Luigi XIV e giunta troppo tardi, aggiunge: "un. de maior damnati, qui iam prenas persolverat, miseraiin ${ }^{1}$.

Senza dubbio, non poteva essergli, e non gli era, nascosto che la piú parte degli individui da lui lodati valeva ben poco. A leggere i suoi scritti panegiristici parrebbe che Napoli avesse allora una nobilta splendida di virtú, di cultura, di dottrina; eppure, informando il padre De Vitry che gli aveva chiesto notizie circa le condizioni degli studî in Napoli, il Vico non celava la realtà : " i nobili sono addormentati da' piaceri della vita allegra ${ }^{2}$. Un suo motto satirico circa quella nobilta, spesso pezzente ma sempre fastosa e capace di soffrire la fame in casa pur di sfogsiare in pubblico con cocchi e altre gale, ci è stato serbato dal suo scolaro Antonio Genovesi ${ }^{3}$. A proposito del letterato duca di Laurenzano formulava la teoria che gli scrittori "nobili » non possono essere se non eccellenti ${ }^{4}$; eppure, tra le sue carte io ho trovato il manoscritto di un libro di quel signore, riscritto da cima a fondo dallo stesso

I Opp., ed. Ferrari, I, pp. 367, 368; e cfr. B. Croce, in Critica, XIX, 377 sger.

2 In Autab., ecc., ed. cit., p. 191.

s Diceva che molti * tiravano le carrozze colle budella .! (ivi, p. 121).

1 In Autob., ecc., ell. cit., pp. $215-6$. 
Vico $^{2}$. Contradizioni e transazioni da pover'uomo, schiacciato dalla miseria e divenuto riguardoso e timido; tanto clie riesce difficile determinare fino a qual punto egli ammirasse a parole e per compiacenza, e fin a qual altro il suo sentimento d'inferiorita sociale si mutasse in effettiva ammirazione per coloro che avevano e ricchezze e dignità e tutto quello che a lni mancava, e che stavano cosi in alto, ed erano $i$ « signori ".

Perché, com'è risaputo, le sue condizioni economiche furono sempre tristissime. Figliuolo di un libraiuccio di Napoli, fu dapprima costretto a recarsi come precettore domestico in un borgo selvaggio del Cilento; poi, tornato a Napoli, tentò invano di ottenere il posto di segretario della città, e, avuta per concorso nel 1699 la cattedra di rettorica, rimase per trentasei anni in quell'ufficio con lo stipendio annuo di cento ducati (lire 425). Invano tento, nel 1723, di passare a cattedra di maggiore importanza: fosse sfortuna, fosse inabilità ( nomo di poco spirito intorno alle cose che riguardano l'utilità", si riconosceva esso stesso) ${ }^{2}$, dové rinunziare a ogni avanzamento universitario. Era costretto, dunque, ad aiutarsi un po' coi lavori letterarî del genere-detto di sopra, e piú ancora con le lezioni private; e non solamente (oltre quella nella pubblica universita) teneva scuola a casa sus, ma saliva e scendeva le altrui scale come insegnante di grammatica a giovinetti, o addirittura a fanciulli. Non fu fortunato nella famiglia: la moglie era analfabeta, senza le virtú delle donne analfabete, incapacissima di curare le piú piccole

1 Bibl. vich., pp. 2i-8.

2 Autol., ed. cit., p. 24. 
faccende domestiche; cosicché il marito doveva farne le parti. Dei figlinoli, una femmina gli morí dopo lunga malattia, e dopo quei lunghi dispendî che inacerbiscono le malattie dei poveri; un figliuolo maschio gli dié grandi dolori ed egli fu costretto a invocare l'intervento della polizia per chiuderlo in una casa di correzione. La sua irrazionale e sublime tenerezza paterna fu tanta, in questa occasione, che al vedere dalla finestra gli uffiziali di polizia, da lui richiesti, i quali venivano a portar via il figliuolo sciagurato ed amato, corse a costui gridandogli: "Figlio mio, sálvati! "1.

Ebbe, invero, animo affettuosissimo: il che si può ritrare, fra l'altro, dall'orazione piena di nobilta e di dolcezza che compose per la morte della sua amica donna Angela Cimini, dagli accenti di pietì e di sdegno che ha nella Scienza nuova per le plebi oppresse, di cui investiga la storia, o per le dolenti figure di Priamo e di Polissena, di cui risente la poesia; e perfino da certi sparsi segni stilistici, come, per es., in quella diguita (la XL) dove ricorda che le streghe, per solennizzare le loro stregonerie, * uccidono spietatamente e fanno in brani amabilissimi innocenti bambini s, e tutto si turba, in modo inopportuno ma significante, per la sorte di quei piccini, che adorna nella commossa fantasia di superlativa amabilita! I maggiori conforti domestici gli vennero dalla figliuola Luisa, colta e poetessa, e dal tigliuolo Gennaro, che lo supplí e poi gli successe nella cattedra. Quando, nell'elogio della contessa d'Althann, accenna sarcasticamente ai filosofi che ragionano passeggiando per gli ameni giardini o sotto i portici dipinti, non nauseati né afflitti dalle " mogli che infantano » e dai a figliuoli che nei morbi

l Villarosa, nelle aggiunte all'Autob. (ed. cit., p. 79). 
languiscono ${ }^{1}$, si sente che parla per diretta esperienz: e che lo pungono ricordi angosciosi della propria vita familiare.

Accade molto spesso, specie ai giorni nostri, di osservare gli uomini di qualche ingegno emanciparsi da questo o quello dei piú umili doveri; e tanto piú bisogna ammirare quest'uomo di genio, che invece li accettò tutti e (peradoperare una parola che il Flaubert disse di sé medesimo), pensando da semidio, visse costantemente da borghese, anzi da popolano. Egli avera preso l'abitudine di leggere, scrivere, meditare e comporre i suoi lavori " ragionando con amici e tra lo strepito de' suoi figliuoli "

La salute ebbe sempre malferma; gli amici lo chiamavano « mastro Tisicuzzo ${ }^{3}$ : debole assai da giovane, straziato in vecchiaia da ulceri alla gola, da dolori alle cosce e alle gambe. Insomma, quel riposo, quell'ozio, quella tranquillita, che altri filosofi goderono per tutta la loro vita, o per lunghi tratti di questa, al Vico maneò sempre. Egli dovette fare da Marta e da Maddalena: travagliandosi per le necessità pratiche sue e dei suoi; travagliandosi insiememente con sé stesso, per adempiere alla missione assegnatagli fin dalla nascita e dare forma concreta al mondo spirituale che gli si agitara dentro.

\section{9}

Non e'è bisogno, dunque, di foggiare o desiderare un Vico eroe, cercandolo nella vita religiosa, sociale e politica, quando il Vico eroe ei sta innanzi, ed è appunto questo: l'eroe della vita filosofica. $\dot{E}$ stato notato da altri

1 Opp., ed. Ferrari, VI, p. 235.

2 Autob., ed. cit., p. 33.

3 In Autob., ed. cit., p. 120. 
che egli ebbe carissima la parola " eroe " e tutti i derivati di essa ( eroismo », "eroico», ecc.); e ne fece continuo uso e svariatissime applicazioni. L'eroismo era, per lui, la forza vergine e strapotente, che appare negli inizî e riappare nei ricorsi della storia. Questa forza egli doveva sentire in sé medesimo, nel lavorare per la verita e nell'aprire, abbattendo ostacoli d'ogni sorta, nuove vie alla scienza. Per questa forza, superate le giovanili incertezze, gli smarrimenti, gli avvilimenti, che talvolta lo fecero cadere in un cupo pessimismo individuale e cosmico (come si vede dalla canzone Affetti d'un disperato), poté sollẹvarsi alla sicura professione di metodo scientifico, che enuncio nel De nostri temporis studiorum ratione, e al suo primo tentativo di applicazione filosofico-storica, rappresentato dal De antiquissima italorum sapientia; e da questo, poi, disfacendo in parte il suo stesso pensiero e ritessendo col resto una nuova tela, giungere al De uno universi iuris principio et fine uno e alla Scienza nuova: « dopo venticinque anni (egli diceva delle scoperte conténute in questa) di continova ed aspra meditazione ».

L'opera, menata a termine da quel povero maestro di grammatica e rettorica, da quel pedagogo che un satirico contemporaneo raffigura \& stralunato e smunto, con la ferula in mano ${ }^{2}$, da quel tormentato paterfamilias, stupisce e, quasi, spaventa: tanta somma di energia mentale vi è condensata. 亡́ un'opera di reazione e di rivoluzione insieme: reazione al presente per riattaccarsi alla tradizione dell'antichità e del rinascimento; rivoluzione contro il presente e il passato per fondare quell'avvenire, che si chiameri poi, cronologicamente, secolo decimonono.

Nel campo della scienza, l'umile popolano diventava

Ivi, p. 120 . 
aristocratico; e quello "stile da signori ${ }^{1}$, che egli falsamente lodava nelle misere scritture dei superbi cavalieri e dei pomposi mitrati del suo tempo, era veramente il suo. Egli aborriva la letteratura galante e socievole, che cominciava a diffondersi dalla Francia in Italia e negli altri paesi d'Europa, i « libri per le dame "2. Ma non meno rifuggiva da quella maniera di trattazioni che si chiamano ora « manuali », e in cui si espongono per filo e per segno definizioni elementari e cose gia da altri accertate: libri che possono giovare soltanto ai giovani ${ }^{3}$, ai quali per altro il Vico già abbastanza si sacrificava nella eerchia della scuola perché dovesse poi sacrificar loro anche qualcosa della propria inviolabile vita scientifica. In questa mirara ad altro pubblico che a giovinetti, cavalicri e dame: quando scriveva, il suo primo pensiero, la sua prima "pratica

- era: *Come riceverebbero le cose da lui meditate un Platone, un Varrone, un Quinto Muzio Scevola?»; e Ia seconda: "Come ricevera queste case la posterita "4. Dei contemporanei, aveva innanzi agli occhi, esclusivamente, la Repubblica letteraria, l'Ordine dei dotti, le Accademie di Europa; un pubblico, a eui non bisognava ripetere ciò che gia era stato trovato e detto nel corso della storia delle scienze e che esso aveva bene a mente, ma porgere soltanto pensieri che fossero reale avanzannento del sapere: non libri voluminosi, ma « piccioli libricciuoli, tutti pieni di cose proprie ${ }^{5}$. Un pubblico ideale, insomma, che ingenuamente egli confondeva talvolta con quello dei dotti di professione e dei critici da riviste letterarie; donde,

1 In Autob., ed. cit., p. 216.

2 In Autob., ed. cit., p. 186.

3 Ordz., ecc., ed. cit., p. 215.

4 Scienza nuova, ed. Niculini, p. 51.

s Oraz., ed. cit., p. 5l. 
poi, le frequenti sue delusioni. I libri brevi, in materia metafisica, sembrava a lui che avessero (come infatti hanno) particolare efficacia, acconciamente paragonata alle meditazioni sacre, " che brievemente propongono pochi punti ", le quali fanno molto piú profitto nelle cose dello spirito cristiano che non « le prediche piú eloquenti e piú spiegate da facondissimi predicatori ' ${ }^{1}$. Per quest'amore alla brevità, fu restio dall'aggravare di troppi libri la repubblica letteraria, che gia non regge sotto il peso; lascio inedite le orazioni, stampò per dovere il De ratione, ed ebbe, infine, a manifestare piú volte ${ }^{2}$ il desiderio che, di tutte le sue opere, sola gli sopravvivesse la Scienza nuova, la quale conteneva condensate e perfezionate tutte le sue indagini precedenti.

All'aristocrazia dell'ideale si accompagnavano nella sua concezione della vita scientifica il piú nobile decoro e la piú profonda lealtà. Dalle sue polemiche si poțrebbe ricavare un intero catechismo cirea il modo in cui si debbono condurre le dispute letterarie. Bisogna (egli diee) non mirare a vincere nella disputa, ma a vincere nella verita; onde voleva che quelle si svolgessero « con sedatissima maniera di ragionare ", perché « chi ha potenza non minaccia e chi ha ragione non ingiuria "; variate tutt'al piú da piacevoli motti, « i quali diano a divedere gli animi de' ragionatori esser placidi e tranquilli, non perturbati e commossi ». Agli avversarî, che movevano obiezioni vaghe, faceva notare: " Il giudizio è in termini troppo generali: e gli uomini gravi non hanno mai di risposta degnato se non le particolari e determinate opposizioni, che loro sono fatte ». Ai medesimi, quando si appellavano al * raftinato

1 Oraz., ed. cit., p. 258.

2 Tra le altre, nella lettera a Celestino Galiani del 18 novembre 1725 (Autob., ed. cit., p. 170-1), e il cui autoğrafo è presso di me. 
buon gusto del secolo, il quale ha sbandito, ecc. ecc.", rispondeva sdegnoso: "Questa è invero una grande opposizione, perché opposizione non è; perché, ritirandosi gli avversarî al tribunale del proprio giudizio, con quel dire di ' codesto che tu dici non ho idea', da avversarî divengono giudici ". Alle autorità non intendeva appoggiarsi, ma neppure le disprezzava; dovendo l'autorita « farci considerati a investigare le cagioni che mai potessero gli autori, e massimamente gravissimi, indur'e a questo o a quello opinare ». E, accusato di avere commesso il medesimo peccato di Aristotele attribuendo errori ai filosofi per poterli con agevolezza confutare, protestava dignitosamente: * Io ni contento del mio poco sapere ingenuo, che essere comparato di mal costume ad un gran filosofo ». Della sua equanimita può dare esempio lo splendido elogio che egli fa di Cartesio, contro il quale pure era rivolto tutto lo sforzo maggiore del suo pensiero. La sua lealtà è attestata dal pronto riconoscere i proprî errori: "Confesso (dice, in un punto, ai critici del Giornale dei letterati) che la mia divisione è viziosa " ${ }^{1}$. "Né gia questo (scrive nella seconda Scienza nuova) dee sembrare fasto a taluni che noi non contenti de' rantaggiosi giudizî da tali uomini dati alle nostre opere, dopo le disapproviamo e ne facciamo rifiuto; perché questo è argomento della somma venerazione e stima che noi facciamo di tali uomini anzi che no. Imperciocché i rozzi ed orgogliosi scrittori sostengono le loro opere anche contro le giuste accuse e ragionevoli anmende d'altrui; altri, che per arventura sono di cuor picciolo, s'empiono de' favoreroli giudizî dati alle loro, e per quelli stessi non piú s'avanzano a perfezionarle; ma a noi le lodi degli nomini grandi hanno ingrandito l'animo di correggere, sup-

1 Si vedano pass. le Risposte, in Oraz., ecc., ed. cit. 
plire ed anco in miglior forma di eangiar questa nostra $»$ '.

Vita scientifica proba, come di serio ricercatore del vero: vita sentimentale commossa e rapita, come di chi giunga a faecia a faceia eol vero a lungo bramato e eercato, ed esulti di poterlo annunziare agli uomini. Di qui la sua alta poesia, che è non già nei rersi, ma nelle prose, e, segnatamente, nella Scienza nuova. "Il Vico è poet: (scrive il Tommaseo): dal fumo di luce, dalle metafisiche astrazioni trae imagini vive: racentando, ragiona e, ragionando, dipinge; e per-le cime de' pensieri non passeggia, ma vola; onde in un suo periodo sorente è piú estro lirico ehe in odi assai ${ }^{2}$. Certo, fossero anche tutte inmaginazioni le sue dottrine, quella nascita che egli deserive della societa, quella rappresentazione delle eta primitive e delle lotte in cui si travagliano e assurgono, splenderebbe ognora, con le sue gigantesche figure, con le sue robuste passioni, eol divino immanente in quegli aspri petti, come un mirabile poema; e il be Sanetis vide infatti nella Scienza nuova l'andamento di un poema, quasi di una nuora Divina commedia. E, come Dante sublime, fu anche piú di Dante severo; e se le labbra del ghibellin fuggiaseo pur si mossero talvolta \& un poco a riso », il Vico leva veramente innanzi alla storia un volto * che giammai non rise ». Del resto, egli che ha avuto tante eensure pel suo stile, non era serittore volgare; anzi, studioso della buona forma e della toseaniti ${ }^{3}$, non meno che sottile estimatore, al dire del Capasso, di vocaboli latini ${ }^{4}$

1 Scienza mova, ed. cit., p. 10.

2 G. B. Vico e il suo secolo (nel vol.: La storia civile nella letteraria, Torino, Lnescher, 1872), p. 104; cfr. un giudizio sul Vico scrittore, ivi, pp.9-10. Piú ampiamente ora, il Nıcolixi, nella introd. alla sua ediz. della Scienza nuova.

3 Autob., ed. cit., pp. 10-11: cfr. Opp., ed. Ferrari, VI, 45, 140.

4 In Autob., ed. cit., p. 120. 
Ia componeva male $\mathrm{i}$ suoi libri, perché la sua mente non padroneggiava tutta la materia filosofica e storica, che aveva accumulata; seriveva confusamente, perché con furore e come in preda a un dèmone: donde, le sproporzioni nelle varie parti dell'opera, nelle singole pagine, nei singoli periodi. Rende taloria immagine di quella bottiglia di cui parla il poeta, piena d'acqua e capovolta di botto, nella quale l'umore, che vorrebbe uscire, tanto s'affretta e intrica per la via angusta, " che a goccir a goccia fuori esce a fatica ». A fatica o a fiotti, disordinatamente. Un'idea che egli sta enunciando, gliene richiama un'altra, e questa un fatto, e il fatto un altro fatto; ed egli vuol dire tutto in una volta, e perciò le parentesi si aprono nelle parentesi, con ritıno spesso vorticoso. Ma quei suoi periodi disordinati, come erano materiati di pensieri originali, cosi solo tutti contesti di frasi possenti, di parole scultorie, di espressioni commosse, d'immagini pittoresche. Egli scrive male, se cosí piace dire; ma di quello "scriver male », del quale i grandi scrittori portano con sé il segreto.

$\mathrm{v}$

L'eroismo filosofico del Vico non si affermò soltanto nella lotta interiore con sé stesso per l'elaborazione della scienza, ma fu sottomesso ad altre e piú dure prove. La posizione mentale, da lui raggiunta, avversa al presente e, sotto specic di reazione, vòlta all'avvenire, lo condannava necessariamente all' incomprensione. É codesta, senza dubbio, la sorte di tutti gli uomini di genio: incompresi intimamente, anche quando la fortuna sociale sembra secondarli ed essi sollevano entusiasmi e trovano in folla scolari e ripetitori. Il motto che, secondo la leggenda, lo Hegel avrebbe pronunziato sul letto di morte ( nno solo 
de' miei scolari mi ha inteso, e questi mi ha frainteso »), esprime a meraviglia tale necessita storica: chi è perfettamente inteso nel suo tempo, muore col suo tempo. Pure, di rado o non mai la sproporzione tra il proprio pensiero e la incomprensione dei contemporanei fu cosi grande come nel caso del Vico. Se altre cagioni d'infelicita non l'avessero tormentato, sarebbe bastata quest' una. Il « desio di laude », che è poi negli animi non rolgari desio di vedere compartecipato, assentito e unicersalizzato negli altri spiriti ciò che a essi sembra vero e buono, rimase sempre per lui un « van desio».

Tanto piú l'incomprensione e l'indifferenza lo angosciavano, in quanto, com'è facile supporre, aveva piena coscienza dell'importanza delle proprie scoperte. Egli sapera che la Provvidenza gli avera affidato una missione altissima; sapeva di esser "nato per la gloria della sua patria, e in conseguenza dell'Italia, perché quivi nato, e non in Marroceo, esso riuscí letterato "'. Allorché mandó fuori la Scienza nuora, gli pareva come di avere dato fuoco a una mina, e ne aspettava da un momento all'altro lo scoppio e il fragore. Non ne segui nulla: la gente non gliene parlava; onde egli seriveva a un amico, dopo qualche giorno: "In questa citta sí io fo conto di averla mandata al deserto; e sfuggo tutti i luoghi celebri per non abbattermi in coloro a' quali l'ho mandata, e, se per necessiti egli addivenga, di sfuggita li saluto: nel quale atto non dandomi essi né pure un riscontro di averla ricevuta, mi confermano l'opinione che io l'abbia mandata al deserto ${ }^{2}$. Egli aveva creduto, addirittura, a un effetto rapido e immediato; e sperato di trovare gli animi pronti e gl'intelletti aperti a ricevere e a fecondare $i$ suoi pensieri,

1 In Autob., ed. cit., p. 48.

2 Lettera al Giacchi, 25 nov, 1725, in Aletcl., ed. cit., p. 175. 
nientemeno che tra $\mathrm{i}$ suoi contemporanei e conoscenti di Napoli: tra i frati occupati a comporre e mandare a memoria prediche verbose, tra i verseggiatori ehe rimavano. sonettuzzi, tra gli avvoeati che scrivevano allegazioni!

Trovò, invece, moltissimi scettici e indifferenti, e non pochi irrisori. Giả il libro sul Diritio universale, quando comparve, venne generalmente " ripreso per oscuretto", come c'informa il Metastasio ${ }^{1}$; e fu poco letto e avventatamente eensurato per le stravaganze che la lettura disattenta e a salti faceva trovarvi in ogni punto ${ }^{2}$. Il padre Paoli, cui l'autore ne aveva donato copia, vi serisse sopra un distico eeliando sull'incomprensibilita clell'opera ${ }^{3}$. Peggio fu per la Scienza nuova: si sa che Nicola Capasso (che pure era dotto uomo e bene affetto verso il Vico), provatosi a leggrerla, credé di avere smarrito ogni sciutilla d'intenclimento, e, buffoneggiando, eorse a farsi tastare il polso dal medico Cirillo ${ }^{4}$. Un erudito senese, nel riferire le impressioni di una sua visita al Vico e della lettura di qualche suo scritto, lo defini stravagante, privo di criterio e seceatore $^{5}$. Un nobile napoletano, interrogato a Venezia dal Finetti circa quel che si pensasse a Napoli del Vico, disse ehe, per un certo tempo costui era passato per uomo davvero dotto, ma che dipoi, per le strane sue opinioni, aveva acquistato fama di squilibrato. «E quando dié fuori la Scienza nuova? », insisté il Finetti. "Oh, allor’a (rispose l'altro), era gia diventato affat to pazzo! ${ }^{6}$. I maldicenti lo

1 Ivi, p. 113.

2 Lettera al Giacchi del 12 ottobre 1720, in Autob., ed. cit., p. 143.

3 Ivi, p. 113.

4 In Autob., ed. cit., p. 119: cfr. p. 76.

5 Lettera di G. N. Bandiera del 1726, ed. dal Nicolini e ristamp. in Critica, XV (1917), pp. 295-97.

'In Autob., p. 119. 
colpivano perfino nella modesta professione da cui traeva il sostentamento, dicendolo a buono ad insegnarc a' giovani dopo aver fatto tutto il corso de' loro studì, cioè quando erano stati da essi già resi appagati del lor sapere »; o, piú insidiosamente, che egli cra adatto non a insegnare, ma a a dar buon indirizzo ad essi maestri "'; c, cioè, riconoscevano la sua superiorità soltanto per farsene un argomento da danneggiallo nella gia cosi stentata sua vita pratica.

\section{VI}

Né alla generale indifferenza e alla leggerezza o alla malignita dei critici potevano formare compenso gli amici e lodatori, che anche al Vico non mancarono. Come sarebbero potuti mancargli, se egli ne faceva una trepida ed attenta cultura artificiale? Si veda, per es., in qual modo coltivasse il cappuccino padre Giacchi. Lodava di costui le " opere ammirabili », " il divinissimo ingegno », « la rar"a sublimita delle meravigliose e divine idec ». Gli annunziava di aver dato a leggere ai letterati della città l'epistola elogiativa ricevuta da lui, e che tutti ne avevano ammirato * il sublime torno del concepire * (eppure egli proprio, rifaceva in latino d'oro le iscrizioni che il Giacchi componeva in un latino fiatesco!) ${ }^{2}$. Gli comunicava, altra volta, che le lodi di un Giacchi avevano destato invidia ed erano state prese da taluni per adulazioni. Eguali fatiche spendeva per propiziarsi l'arcivescovo di Bari, Muzio di Gacta, un vanitoso, tutto pieno del proprio merito (negli Elogi del Gimma fa perfino lodare la sua avvenenza), e che non sapeva parlare se non di sé stesso, autore di un panegirico

1 Autob., 1. c.

2 Eurono pubblicate da me in Napoli notriliss., XIII (1904), f. I, e di hus vo in treondu sitppl. alla Bihl. vich., pp. 70-2. 
di papa Benedetto XIII, pel quale, lodato e rilodato dal Vico, non si saziava mai, e provocava, anzi chiedeva espressamente, nuove lodi. E il Vico a inaffiarlo pazientemente della linfa desiderata: "la maravigliosa opera di V. S. I. »; il suo « dire da signore »; le « digressioni demosteniche »; l'eloquenza, che fu la favella filosofica, con la quale parlarono gli antichi accademici greci, tra i latini Cicerone, e " tra gl'italiani niun altro che V. S. I. »! All'avvocato Francesco Solla, che gli era stato scolaro e si era poi ritirato in provincia, insinuava che la sua Scienza nuova aspettava che egli fosse tra i poclissimi forniti d'alto intendimento, che volessero riceverla con mente sgombra di tutti i pregiudizî circa i principî dell'umanità ${ }^{\text {. }}$ Erano artifizî ingenui, fanciullaggini pietose, con le quali procurava di dare un'illusoria soddisfazione al suo bisogno di riconoscimento e di lode, e un calmante ai suoi nervi eccitati. Ma anche a questo modo raccoglieva frutti assai poveri. Nelle lettere del Giacchi, non è parola che provi clic costui avesse intesa una sola delle dottrine vichiane o che almeno le avesse considerate con serio interesse. Monsignor di Gaeta, dopo molti giri di frasi, gli confessa di avere "piú ammirate che intese " le opere di lui ; e, certamente, non le aveva neppur lette, tutto occupato ad ammirare la propria prosa. Il Solla, nel quale il Vico sembrava riporre tante speranze, giudicava l'orazione per la morte di donua Angela Cimini cosa superiore a tutte le altre opere dell'autore e alla stessa Scienza nuova. Un simile incauto complimento rivolgeva al Vico un altro ammiratore, pur caldo e affettuoso, l'Esteban ${ }^{3}$. Lodi generiche o banali gli giungevano talvolta (ma piú spesso per-

1 In Autob., ed. cit., p. 202.

2 Ivi, p. 251.

3 Ivi, pp. $195-6$. 
duravano, ostinati, la trascuranza e il silenzio), in ricambio di alcuno dei tanti esemplari delle proprie opere, che inviava non solamente ai letterati di Napoli, ma a quelli di Roma, di Pisa, di Padova, anzi di Germania, di Olanda, d'Inghilterra: ne mandò, perfino, a Isacco Newton ${ }^{1}$. Egli ottenne, tutt'al piú, di farsi considerare ertudito tra centinaia di eruditi e letterato tra migliaia di letterati: dotto uomo, insomma; ma niente altro.

Senza dubbio, il Vico ebbe, tra i modesti, tra gli oscuri, tra i giovani, schietti ammiratori. Di costoro era il poeta, poi oratore sacro, Gherardo de Angelis; i gia ricordati Solla ed Esteban; il frate Nicola Concina di Padova; e altri pochi. Ma, se il loro affetto era grande, la loro intelligenza era scarsa. Anche il Concina confessava, in mezzo al fervore dei suoi entusiasmi, di non intendere troppo bene: "Oh quanti fecondissimi e sublimissimi lumi vi solıo per entro! Cosí avessi io talento da farne uso e da comprendere il fondo ed il mirabile artificio, che parmi alquanto di ravvisare! "2. Il miglior ufficio, che codesti amici potessero adempiere, era di lenire con parole buone, se non con intima corrispondenza di pensieri, l'animo esacerbato del Vico. Cosi faceva l'Esteban, concludendo Ia lettera nella quale procura di rimediare a quel che gli era scappato dalla penna a proposito dell'orazione per la Cimini, e ripeteva frasi che aveva dovuto cogliere sulla bocea del maestro: "Vivete sicuro che la Provvidenza, per canali da V.S. non immaginati, fara sorgere a V.S. una fonte perenne di glorie immortali! " ${ }^{3}$. Il gesuita padre Domenico Lodovico (autore del distico, che si legge sotto il ritratto del Vico), riceruta la seconda Scienza

\footnotetext{
1 Autub., ed. cit., p. 55.

In Autob., ed. cit., p. 231.

3 Ivi, p. 205.
} 
uuova, mandò all'autore, con pratico senno, un po' di vino della cantina c un po' di pane del forno della casa gesuitica della Numziatella, con una graziosa letterina nella quale lo pregava di accettare « codeste cosucce, comeché scmplici, quando né pure il bambino Gesú rifiuta le rozze offerte de' rustici pastorelli ». E gli suggeriva di aggiungere nella simbolica dipintura che precede l'opera, accanto all'alfabeto, un piccolo nano in atteggiamento di chi ammirando ammuta come il montanaro di Dante, scrivendovi sotto « con significante dieresi » il nome: Lodo-vico! ". Tra i tanti giovani della sua scuola, crano alcuni, tutti pieni della dottrina di lui, pronti a difendere il maestro a spada tratta ${ }^{2}$; ma si sa che cosa valgano codesti entusiasmi di giovani. Se quegli scolari avessero penetrato davvero le dottrine o qualche parte delle dottrine vichiane, se ne sarebbero vedute le tracce nella letteratura e nclla cultura della generazione che seguí al Vico; e, invece, non ne fu nulla o quasi. Appena qualche sentenza, qualche affermazione storica, qualche concetto isolato e superficialmente inteso fu ripetuto a Venezia dal Conti, a Padova dal Concina, in Ispagna da Ignazio Luzán (il quale aveva dimorato a Napoli negli anni della pubblicazione della Scienza nuova) ${ }^{3}$, e qualche cosa di piú nella patria dell'autore, dal Genovesi e particolarmente da Ferdinando Galiani.

Gl'invidi, i leggieri, i pettegoli, i calunniatori, gl' inintelligenti eccitavano nel Vico scoppî di collera violenta. Di questo suo peccato si confessa nell'autobiografia, dicendo che * con maniera troppo risentita inveiva contro o gli errori d'ingegno o di dottrina o mal costume dei letterati suoi emuli, che doveva con cristiana carita, e da vero fi-

1 In Autob., ed. cit., pp. 213-4.

2 Ivi, p. 121.

3 Ivi, p. 122. 
losofo, o dissimulare o compatirgli „'. Ma, in fondo, quel peccato non gli spiaceva: al pari di Dante, vi trovava qualche bellezza. L'orazione per la Cimini contiene una specie d'inno alla collera, alla * collera eroica ", "che negli animi generosi co' suoi bollori turbando e dall'ino confondendo ogni mal nata riflessione della mente, da cui nasce la razza vile della fraude, dell'inganno, della menzogna, fa ella gli eroi aperti, veritieri e fidi, o sí, interessandoli della verita, li arma forti campioni della ragione incontro ai torti ed alle offese " ?

Benché nello scrivere si guardasse « a tutto poterc » ral cadere in quella passione ${ }^{3}$, la collera si sente tumultuare mal repressa nelle lettere private, in tutte quelle punte contro $\mathrm{i}$ * dotti cattivi ", che « amano piú l'erudizione che la verita », contro il comune degli uomini che è " tutto memoria e fantasia ", e via dicendo. Nella conversazione poi, era, a quel che sembra, mordacissimo. Quando, nel 1736, Damiano Romano pubblicò ụn libro contro la tesi vichiana relativa alle dodici tavole, il. Vico (racconta il Romano medesimo), sebbene vi fosse stato trattato coi titoli di "dottissimo » e di « celeberrimo » e con ogni altra dimostrazione di reverenza, "ci addentó in maniera che fu di ribrezzo e di orrore a chiunque vi si trovò presente ", vedendo egli di malissima voglia che "un garzone come noi si fusse con lui cimentato ${ }^{4}$. Ma agli scoppî di collera si alternavano le ricadute nella piú profonda tristezza. In un sonetto, egli si dice oppresso da quel fitto " che l'ingiusto odio altrui creò sovente », onde si era ap-

\footnotetext{
1 Ivi, pp. 96, 120-1.

O Opp., el. Ferrari, VI, p. 254.

:Autob., ed. cit., p. 76.

4 In Autob.; pp. 120-1.
} 
partato dal consorzio umano a vivere solo con sé stesso. Da quel torpore si riscoteva, talvolta, per qualche istante:

Poi ricaggio in me stesso, e da mie gravi cure sospinto a tornar là dov'era, di me, non per mia colpa, ho da dolermi ${ }^{1}$.

VII

Eppure, fra tanti tormenti e contrarietà e delusioni, in mezzo a questa tristezza che veniva frequente a ricoprirlo dei suoi neri veli, il Vico provó una delle piú alte felicità dell'nomo: quel « vivere di meditazione scevra e pura di passione, ché allora senza la compagnia tumultuosa e grave del corpo vive veramente l'uomo solo.... "; quella vita di sicuro possesso, perché « medesimata con l'anima, sempre presta e presente, che gli dimostra il suo essere fisso nell'Eterno che tutti i tempi misura, e spaziante nell' infinito che tutte le finite cose comprende; e sí il colma di una eterna immersa gioia, non in certi luoghi invidiosamente né in certi tempi avaramente ristretta, ma che senza uggia di emulazione, senza tema di scemamento, per ciò unicamente in esso lui accrescere si potrebbe se ella fosse tuttavia a piú e piú umane menti comunicata e diffusa ${ }^{2}$. Della verità raggiunta non dubito mai, pur continuando sempre a elaborarla: sopra il sistema presentato nel libro del Diritto universale la sua mente (egli dice) « riposava sodisfatta ${ }^{3}$. Le fatiche, e gli stessi dolori che avera cosí acerbamente sofferti, gli erano cari, perché attraverso di essi era pervenuto alle sue scoperte:

1 In Autob., ed. cit., p. 325.

¿ Opp., ed. Ferrari, p. $2 t \bar{\gamma}$.

3 Lettera al Giacchi del 12 luglio 1720, in Autob., ed. cit., p. 138. 
"Benedico ben venticinque anni da me spesi nella meditazione di siffatto argomento, ed in mezzo le avversiti della mia fortuna e le remore che mi facevano gli esempli infelici degl'ingegni, che han tentato delle nuove e gravi diseoverte.... ${ }^{2}$. Come poteva non benedire quelle fatiche e quei dolori e quelle avversità, se ogni qual volta si sollevava dal tumulto passionale dell' uomo empirico e dalle lotte dell'uomo pratico, la sua mente gli mostrava la necessita ineluttabile e di quanto egli aveva operato e di quanto aveva sofferto, e l'una e l'altra necessita strette in modo tra loro da formarne una sola e indivisibile?

La sua stessa dottrina filosotica gli porgeva dunque la medicina del male, e promoveva nel suo animo la catarsi liberatrice: quella dottrina che aveva per centro l'idea della Provvidenza immanente o, come si disse poi, della necessità storica. "Sia pur sempre lodata la Provvidenza, che quando agl'inferni occhi mortali sembra ella tutta severa giustizia, allora piú che mai è impiegata in una somma benignità! Perché da questa opera io mi sento aver vestito un nuovo uomo e provo rintuzzati quegli stimoli di piú lamentarmi della mia avversa fortuna, e di piú inveire contro alla corrotta moda delle lettere clie mi ha fatto tal'avversa fortuna; perché questil moda, questa fortuna mi hanno avvalorato e assistito a lavorare quest'opera. Anzi (nou sara per avventura egli vero, ma mi piacercbbe che fosse vero), quest'oyera mi ha informato di un certo spirito eroico, per lo quale non piú mi perturba alcun timore della morte e sperimento l'animo non pii curante di parlare degli emoli. Finalmente, mi ha formato come solra un'alta adamantina ròcca il giadizio

1 Lettera al card. Corsini del 15 dicembre 1725, in Autol., ed. cit., p. 178 . 
di Dio, il quale fa giustizia alle opere d'ingegno con la stima dei saggi ", degli uomini cioè di altissimo intendimento, di erudizione tutta propria, generosi e magnanimi, " intenti a conferire opere immortali nel comune delle lettere ", che " sempre e da per tutto furono pochissimi "1. La Provvidenza gli mostrava, dunque, la necessità di tutto cio che gli era accaduto e ancora gli accadeva nella vita, e, inculcandogli la rassegnazione, gli prometteva la Gloria.

\section{VIII}

Cosí l'uomo collerico diventara períno tollerante: di quella tolleranza, di quella indulgenza superiore che non è da confondere col volgare tollerantismo. L'Universita, nella quale aveva sperato fare avanzamento e verso cui avera rivolto il pensiero nel comporre le prime opere, non aveva voluto sapere di lui; ed egli si era tutto ritirato in sé stesso a meditare la Scienza mova. Dunque (dicẹra con sorriso in cui si sente ancora alcunché di amaro), questa mia opera io la debbo all' Università, che, riputandomi immeritevole della cattedra e non volendomi « occupato a trattar paragrafi ", mi ha dato l'agio di meditarla: " posso io avergliene piú grado di questo?" ". Un amico, il forentino Sostegni, in un sonetto a lui indirizzato, usciva in parole di biasimo contro la città di Napoli, che aveva tenuto in poco conto il suo gran figlio. $\mathrm{E}$ il Vico, nella risposta, giustifica con nobili parole la patria, dura con lui perché molto da lui aspettava e molto areva voluto ottenerne:

1 In Autol., ed. cit., pp. 120-1.

2 Lettera al Giacchi del 25 novembre 1725, in Autob.; ed. cit., p. 134. 
Severa madre non vezzeggia in seno figlio, che ne fia poscia oscura e vile; ma grave in viso ancor l'ode e rimira '.

Da questa condizione di spirito nacque l'Autobiografia: opera che è stata mal giudicata e del tutto fraintesa dal Ferrari, il quale vi biasima il teleologisino dominante e vi lamenta la mancanza di una spiegazione "psicologica » della vita del Vico ${ }^{2}$. Come se il Vico medesimo non avesse professato che l'aveva scritta « da filosofo $!^{3}$. E che cosa significa scrivere da filosofo la vita di un filosofo, se non intendere l'oggettiva necessita del suo pensiero e scorgerne gli addentellati anche dove all'autore, nel momento che lo penso, non apparivano del tutto chiari? Il Vico " meditò nelle cagioni cosi naturali come morali, e nell'occasioni della fortuna; meditò nelle sue ch'ebbe fin da fanciullo o inclinazioni o avversioni piú ad altre spezie di studî che ad altre; meditò nell'opportunitadi o nelle traversie onde fece o ritardò i suoi progressi; meditó, finalmente, in certi suoi sforzi di alcuni suoi sensi diritti, i quali poi avevangli a fruttare le riflessioni, sulle quali lavorò l'ultima sua opera della Scienza nuova, la qual appruovasse tale e non altra aver dovuto essere la sua vita letteraria " ${ }^{4}$. L'Autobiografia del Vico è, insomma, l'applicazione della Scienza nuova alla biografia dell'autore, alla storia della propria vita individuale; e il metodo ne è, quanto originale, altrettanto giusto e vero. Che poi il Vico riuscisse solo in parte nel suo assunto, e, cioè, non potesse fare la critica c la storia di sé stesso come sono in

1 In Autob., ed. cit., p. 325.

* Nell'introd. al IV vol. delle Opere.

3 Autob., ed. cit., p. 62.

L. c. 
grado di farla i critici e gli storici odierni, e altrimenti saranno quelli futuri, è troppo ovvio perché vi si debba insistere. - L'Autobiografia termina anch'essa-con una benedizione alle avversità, un riconoscimento deĺla Provvidenza e una certezza di fama e di gloria.

IX

Negli ultimi anni di sua vita il Vico, aggravato dalla vecchiaia, dalle domestiche cure e dalle malattie, « rinunziò affatto agfi studî̀ $\star^{2}$.

Da la tremante man cade il mio stilo e de' pensier s'è chiuso il mio tesauro?,

esclamava in due rersi, pieni di lacrime, di un sonetto del 1735. Preparò allora, per una possibile ristampa, le aggiunte e correzioni alla seconda Scienza nuova, e le incorporò nel definitiro manoseritto dell'opera; pensò per un momento di mettere a stampa l'operetta De cequilibrio corporis animantis, composta molti anni prima c che andò poi smarrita ${ }^{3}$; adempié ancora a qualche obbligo di uffizio, come, nel 1738, all'orazione per le nozze di re Carlo Borbone. Ma già nel 1736 o nel 1737 il figliuolo aveva cominciato a sostituirlo nella scuola, c nel gennaio 1741 riceveva definitivamente la cattedra dalla quale il padre si ritirava 4 Viveva il Vico tra i suoi, come un vecchio soldato exceta militia, nel ricordo delle battaglic combattute, nella coseienza del dovere compiuto. Il buon figliuolo gli faceva,

1 Autol., ed. cit., p. 75.

2 In Autob., ed. cit., p. 326 (sonetto del 1735 per le nozze di Raiinondo de Sangro).

3 Autob., ed. cit., 75: cfr. Bill. vich., pp. 38-9, Sec. suppl., p. 6.

1 In Aulob., ed. cit., p. 123. 
ogni giorno, qualche ora di lettura dei classici latini da lui piú amati e studiati un tempo. $\mathrm{E}$, in questo suo tramonto, gli fu risparmiato, almeno, il tormento dei tormenti: qucllo che straziò negli ultimi anni di vita un filosofo tanto di lui piú fortunato, Emanuele Kant, ansioso di dare séguito e compimento al suo sistema filosofico e consumantesi in una sterile lotta coi pensieri che gli sfuggivano e le parole che non più gli obbedivano. Il Vico aveva detto tutto ciò che dovera dire, e conobbe da sé stesso, quale grande storico di sé stesso, il momento in cui la Provvidenza aveva terminato in lui l'opera sua, chiudeva il tesoro dei pensieri che gli aveva cosi largamente aperto per tanti anni c gli comandava di deporre la penna. 
.

$-$ 


\section{LA FORTENA DEL VICO:}

La narrazione delle vicende alle quali andò soggetta la fitma del Vico non dev'essere sostituita o frammischiata all'esposizione e gindizio dol pensiero vichiano, perdendo di rista la storia della filosofia propriamente detta o turbandola con la storia della cultura². Ma anche quando poi si passi a questa seconda storia, bisogna guardarsi da un altro genere di errore: dalla pretesa di giungere a determinare, mercé quella narrazione, se l'opera del Vico fosse o no culturalmente utile, e quanti gradi di utilita le si debbano riconoscere. Siffatta indagine è priva di significato e la corrispondente misurazione impossibile a eseguire; perché (se ben si consideri) un unico discepolo può valere le decine e le centinaia, un effetto solo prodottosi dopo secoli compensare un'efficacia ritardata per secoli, un oblio immeritato riuscire altrettanto memorabile e ammonitiro quanto una fama meritatissima, e una mede-

* Restringo in breve i principali risultati delle ricerche da me fatte sull'argomento ed esposte nella Bibliografia vichiama e nei due annessi Supplementi (cfr. in questo volume, p. 342), ai qnali lavori rimando per maggiori particulari e per la documentazione delle cose che qui si affermano.

1 Si veda sopra, pp. 249-50. 
sima verita, scoperta due volte in modo indipendente, da questa stessa duplicazione e apparente superfluita ricevere come il crisma della sua ineluttabile necessità. L'opera del Vico (si è concluso di solito) fu del tutto inutile, perché apparsa fuori tempo ossia troppo presto, e rimasta sconosciuta - giunta a notizia quando non poteva insegnare piú nullat. $E$, col dire ciò, si è blasfemato contro la storia, la quale non ammette nulla d'inutile ed è sempre, in ogni sua parte, opera (avrebbe detto il Vico) della «Provvidenza », alle cui ampie utilità non è lecito applicare piccole misure umane, corte di una spanna.

Ebbe il Vico rinomanza, lettori, intenditori e seguaci nel corso del secolo decimottavo? Si è risposto, con pari risolutezza, no e si ; e, a provare la risposta aformatira, si sono andati raccogliencio con molta diligenza i ricordi che del nome e dclle dottrine del Vico si trovano sparsi negli scritti di quel secolo, accumulando sospetti e indizî su tracce inconfessate dolle sue idee, che si scorgerebbero in libri italiani e stranicri. Ma un pensatore come il Vico non si può dire propriamente conosciuto se non quando di lui sia stato còlto il pensiero fondamentale e risentito lo spirito animatore. Ora la maggior parte dei fatti arrecati a documento dell'efficacia dell'opera sua concernono dottrine particolari, che, avulse dal complesso, furono accettate o contestate né piú né meno di quelle di qualsiasi altro critico ed erudito o dicitore di paradossi del tempo suc. Tale è il caso, in primo luogo, della teoria circa l'origine della legge delle dodici tavole, discussa nella poIemica che si agitò fra Bernardo Tanucci e Guido Grandi dal 1728 al 1731, oppugnata nel 1736 da Damiano Romano, accolta in Francia nel 1735 dal Bonamy e rammentata nel 1750 dal Terrasson; delle nuove interpretazioni storiche circa i primi tempi di Roma, ricordate dallo Clastellux, seguite e svolte dal Duni e, attraverso costui, sfruttate dal 
Du Bignon; delle ipotesi sulla preistoria e sulle origini dell'umanita, adoperate e alterate dal Boulanger in Francia e da Mario Pagano in Italia; dei concetti storici e politiei, e di quelli sulla poesia e sul linguaggio che si trovano presso il Galiani, il Pagano, il Cesarotti e qualche altro.

Questione piú sostanzialo era quella del metodo di studiare e giudicare le istituzioni politiche e le leggi; per la qual parte il Montesquieu fu messo a paragone col Vico e accusato di essersi valso largamente della Scienza nuova senza citarla. Ė ormai accertato che nel 1728 Antonio Conti in Venezia consigliò al futuro autore dell'Esprit des lois (come risulta dai diarî di quest'ultimo) di comprare a Napoli il libro del Vico: consiglio che fu certamente messo in atto quando il Montesquieu si lecò a Napoli l'anno dopo, per'ché un esemplare della scienza nuova, nell'edizione del 1725 , si serba ancora nolla biblioteca del castello de li Brède. Ma ingegno troppo diverso rispetto al Vico, e troppo meno profondo, era quello dello scrittore francese, da trarre vitale nutrimento da un'opera come la Scienza nuova; e i vestigi d'imitazione, che si è creduto di scorgere nell'Esprit des lois, sollo assai contestabili e, in ogni caso, di scarsil importanza. Deve dirsi, per altro, che il merito generalmente attribuito al Montesquieu, di avere introdotto l'elemento storieo nel diritto positivo, prendendo per tal modo a considerare in guisa veramente filosofica (come poi scrisse lo Hegel) la legislazione, quale momento dipendente di una totalita in rapporto a tutte le altre determinazioni che formano il carattere di un popolo o di un'epoca; - questo unerito, in ordine cosi di tempo come di eccellenza, spetta invece al Vico.

Come il Montesquieu per la scienza della legislazione, cosí il Wolf per la questione omerica, fu sospettato di essersi giovato tacitamente delle speculazioni vichiane. Ma il Wolf, quando nel 1795 dié fuori i Prolegomena ad Home- 
rum, ignorava, almeno direttamente, la Scienza nuova, che non conobbe se non di nome nel 1801 e di fatto l'anno dopo, pel dono che di quel libro gli fece il Cesarotti. $\grave{E}$ da notare per altro ehe i concetti del Vico circa il carattere barbarico e la mancanza di riposta sapienza nell'epos omerico erano (forse per opera del Galiani) divulgati nel 1765 dalla Gazette littéraire de l'Europe del Suard e dell'Arnaud; e, meglio ancora, che la Scienza nuova era conoseiuta e adoperata dal filologo e archeologo danese Zoega (il quale la cita in un suo saggio su Omero, composto nel 1788 sebbene pubblicato assai piú tardi); c elic con lo Zoega tellevit carteggio lo Heyne, il quale aceusò poi il Wolf di avere attinto alle sue lezioni per la teoria presentata nei Prolegomena (e, in verità, sin dal 1790 manifestava l'idea di una genesi graduale dei poemi omerici); e, infine, che quelle teorie gia si profilavano nel Wood e in alcune memorie del Merian. I concetti vichiani con o senza il nome del loro autore erano dunque penetrati in qualehe misura nell'ambiente filologico tedesco; e il Wolf ne ebbe indubbiamente un certo sentore indiretto. $\mathrm{E}$, in ogni caso, resta sempre, anche qui, il fatto riconosciuto da tutti coloro che hanno studiato la questione: che la teoria omerica, cosi come si trova esposta dal Wolf, dovrebbe dirsi non wolfiana ma vichiana, perché tale è veramente in quasi tutti i suoi tratti fondamentali. Del resto, il Wolf, filologo di gran lunga superiore al Vico ma anch'esso pensatore assai minore, non era in grado d'intendere le motivazioni ideali che avevano condotto il suo predecessore a quella dottrina intorno a Omero; com'è chiaro dall'articolo, alquanto superficiale, che nel 1807 vi serisse intorno.

Certamente a Napoli, nel secolo decimottavo, $f u$ in molti una confusa coscienza della grandezza dell'opera vichiana; ma in che propriamente questa grandezza consistesse nou si sapeva determinare, perché facevano aneora 
difetto l'esperienza e la preparazione adeguate. E fuori d'Italia, e in Germania in particolare, dove questa preparazione c'era, o almeno ce n'era assai di piú, l'opera del Vico rimase generalmente sconosciuta, in parte per il discredito in cui erano caduti sin dalla fine del seicento $i$ libri italiani, in parte per le difficolta che lo stile del Vico offriva agli stranieri. Quando la Scienza nuova capito tra le mani di nomini atti a comprenderla, sembra come se il caso si divertisse a impedirne loro la seria lettura e l'intelligenza. Lo Hamann si procurò la Scienza nuova da Firenze nel 1777 , in un tempo in cui si occupava di economia e di fisiocrazia, immaginando che vi si trattassero tali materie; e rimase deluso quando, nella scorsa che le dette, si arvide di avere innanzi una selva di ricerche filologiche, eseguite per giunta con scarsa acribia. Il Goetle l'ebbe a Napoli, nel 1787, con grandi raccomandazioni, dal Filangieri e la portò seco in Germania e nel 1792 la prestava al Jacobi; ma solo per una felice combinazione, piuttosto che per una vera conoscenza o per un chiaro intuito, avvicinò il nome del Vico a quello dello Hamann. Lo Herder (che anch'esso conobbe l'opera del Vico non for'se nel 1777 mercé l'accenno fattogliene dallo Hamann nel loro carteggio, ma piuttosto nel suo viaggio d'Italia nel 1789), ne discorse nel 1797 in termini affatto generici e senza avvertire nessuno dei molteplici rapporti clie al Vico lo stringevino, in ispecie nelle dottrine sul linguaggio e sulla poesia.

I soli che nel secolo decimottavo veramente penetrassero la tendenza fondamentale del Vico e, pur senza volerlo, ne riconoscessero la gelluina grandezza, furono (a nuova conferma della salda contestura spirituale del cattolicesimo) gli avversarî cattolici, che egli, allora, ebbe in buon numero: il Romano, il Lami, il Rogadei, e sopra tutti, il Finetti. Videro costoro che il Vico, nonostante i suoi fermi 
propositi di ortodossia religiosa, coltivava un'idea delli Provvidenza affatto difforme da quella della teologia cristiana, e di Dio faceva continua menzione a parole, ma non lo lasciava poi operare effettivamente, come Dio personale, nella storia; - che distaccava con taglio cosí netto storia profana e storia sacra da giungere a una dottrina affatto naturale e umana delle origini della civiltà (mercé lo stato ferino) e di quelle della religione (mercé il timore, il pudore e l'universale fantastico), laddove la dottrina tradizionale cattolica ammetteva una certa comunicazione tra la storia sacra e la profana, e nella religione e civilti pagana riconosceva il lievito operante di una qualche notizia, sia pur vaga, della primitiva verità rivelata; - che, pure protestando di aceogliere e rafforzare l'autorita della Bibbia, egli la millava e scrollava in molti punti; - che la sua critica alla tradizione storica profana, condotta con spirito superbo di ribellione al passato, poteva aprire l'adito a dannosissimi abusi, perché istigava ad applicare il medesimo spirito e metodo alla storia sacra, come fece poi il Boulanger ${ }^{1}$. Un'invettiva, insomma, nella quale erano gia accuratamente indicate tutte le parti che dovevano dipoi entrare a comporre il grandioso elogio che il secolo decimonono avrebbe indirizzato al Vico. Nacque per tal moulo tra gli uomini di chiesa una certa diffidenza verso questo autore; di che, tra l'altro, fu effetto piú tardi, al tempo della restaurazione, la polemica antivichiana del vescovo Colangelo, preceduta da un giudizio del regio censore Lorenzo Giustiniani, che diceva la Scienza nuova: un «libro il quale diede luogo a segnare un'epoca molto infelice in Europa \%

1 La critica dei cattolici contro il Vico porse materia a un libro assai istruttivo del Labanca: cfr. piú oltre in questo volume, p. 339. 
Quasi a contrasto, tra $\mathbf{i}$ giovani che in Napoli, sulla fine del secolo decimottavo, coltivavano con ardore gli studî sociali e politici e si accingevano all'opera attiva dellà imminente rivoluzione, il Vico cominciò a essere considerato come scrittore anticlericale e anticattolico, e sorse la leggenda (ricordata in altra parte di questo volume ${ }^{1}$ ) che il Vico avesse di proposito e per accorgimento reso oscuro il suo libro per salvarsi dalla censura ecclesiastica. Quei giovani presero a leggere e a vantare la Scienza nuova; disegnarono di ristamparla, perché era divenuta rara, con le altre opere dell'autore e con gli scritti inediti; prepararono lavori espositivi e critici sul sistema filosofieo e storico del Vico; taluno, come il Pagano, si provò a riclaborarlo mescolandolo con le idee del sensismo francese, e tal altro, come il Filangieri, benché molto lo ammirasse, non ne fu distolto dai sogni del piu roseo riformismo; nel 1797 il tedesco Gerning, che capitò a Napoli, notò questo fervore di studî intorno al Vico e augurò una traduzione o almeno un estratto tedesco della Scienza nuova. E quando la caduta della repubblica napoletana del 1799 spinse quei giovani (quelli, tra essi, che scamparono dalle stragi e dai patiboli della reazione borbonica) agli esilî nell' Italia superiore e specialmente in Lombardia, la fama del Vico cbbe i suoi primi ardenti apostoli e missionarî. Vincenzo Cuoco, Francesco Lomonaco, Francesco Salfi e altri patrioti meridionali fecero conoscere la Scienza nuova il Monti, che ne toccò nella sua prolusione universitaria di Pavia del 1803; a Ugo Foscolo, che ne accolse parecchi pensieri nel carme dei Sepolcri e nei saggi di critica; ad Alessandro Manzoni, che doveva poi istituire nel $\mathrm{Di}$ scorso sulla storia longobarda un celebre raffronto tra il Vico e il Muratori; e ad altri minori. Il Cuoco informo

I Si veda sopra, pp. $28 \overline{\text { - }} 6$. 
intorno al Vico il Degérando, che allora lavorava alla sua Histoire comparée des systèmes philosophiques; un altro esule, il De Angelis, metteva la Scienza nuova tra le mani di Giulio Michelet; il Salti discorreva del Vico negli articoli della Revue encyclopédique e in volumi ed opuscoli scritti in francese. Anche per suggerimento di quei napoletani, fu nel 1801 a Milano ristampata la Scienza nuova; e altre edizioni e raccolte di opere minori vichiane non tardarono a comparire. Per tali vicende, e in quel primo decennio del secolo decimonono, il Vico, da reputazione quasi esclusivamente municipale e napoletana, pervenne a reputazione nazionale e italiana.

Senonché, conforme alle loro personali disposizioni e alle tendenze del tempo, il primo e principale ammaestramento che i patrioti studiosi del Vico trassero dal suo pensiero, fu politico o di filosofia politica; e cioè, la critica di quel giacobinismo e di quel filogallismo che avevano fatto cosí cattiva prova negli avvenimenti del 1799. Il pensiero del Vico li guidò a concetti piú concreti, e generò un'opera di capitale importanza, il Saggio storico sulla rivoluzione napoletana (1800) di Vincenzo Cuoco. Similmente, alcuni decennî dopo, il Ballanche, nei suoi Essais de palingénesie sociale (1827), scriveva che il Vico, se fosse stato noto in Francia nel secolo decimottavo, avrebbe esercitato un'azione moderatrice e benefica sulle rivoluzioni sociali che seguirono. Un altro particolare aspetto del Vico, la riforma ch'egli aveva iniziata della metodologia storica e della scienza sociale a servigio della storia, fu avvertito e lumeggiato dall'archeologo Cataldo Iannelli nel libro: Sulla natura e necessità della scienza delle cose e delle storie umane (1818). Il Foscolo principalmente, e coloro che da lui presero ispirazione, fecero penetrare nella critica e storia letteraria qualcosa delle concezioni vichiane sulla interpetrazione storica della poesia. 
Invece, in Germania, il Jacobi, che aveva letto il $D e$ intiquissima, si collocava subito nel centro del pensiero tilosofico vichiano, scorgendo e additando fin dal 1811, nel suo libro Ueber den göttlichen Dingen und ihrer Offenbarung, lo stretto rapporto tra il principio della conversione del vero col fatto, e la teoria kantiana che non si possa perfettamente concepire e intendere se non ciò che si è in grado di costruire; dalla quale un sol passo conduceva poi (egli notava) al sistema dell'identità. La medesima cosa riconobbe il Baader, che trovava in questo sistema la conferma e il fondamento del prinsipio enunciato dal Vico. Ma la buona traduzione della Scienza nuova, pubblicata nel 1822 dal Weber, non sembra avesse fortuna; né del Vico sembra che conoscesse nulla lo Hegel, che tante affinitá sostanziali e formali (in ispecie nella Fenomenologia) ha col pensatore napoletano e al quale doveva essere rimproverata la mania triadica, come già il cattolico Finetti aveva rimproverato il Vico di star sempre « sulla regola del tre ». Né i riscontri delle nuove dottrine filologiche tedesche del Niebuhr, del Mïller, del Böckh e di tanti altri con quelle vichiane furono volentieri messi in luce; e caratteristico è l'atteggiamento del Niebuhr, il quale, conoscesse o no l'opera del Vico quando pubblicò la prima edizione della sua Römische Geschichte, la conobbe senza dubbio dipoi per comunicazione che gliene f'ece il Savigny e per l'articolo di confronto: Vico und Viebuhr, pubblicato nel 1816 dallo svizzero Orelli; eppure continuò a tacerne per non si sa quale dispregio o dispetto, nel che fu imitato poco lodevolmente dal Mommsen.

In Francia, la divulgazione del pensiero del Vico si dovette al Michelet, che ne tradusse le opere e che ancora negli ultimi suoi anni chiamava l'Italia: \& cette seconde mère ct nourrice qui, jeune, m'allaita de Virgile, et, mn̂r, me nourrit de Vico, puissants cordiaux qui tant de fois ont renou- 
velé mon ceeur ». E il Michelet, per il primo o tra i primi, scrisse che il Vico non era stato inteso nel secolo decimottavo, perché parlava al decimonono. Fecero corona al Michelet,.oltre il Ballanche gia ricordato, il Jouffroy, il Lerminier, lo Chateaubriand, il Cousin (a parecchi dei quali non isfuggirono i rapporti tra il Vico e quella filosofia tedesca che il Cousin per l'appunto divulgara allora in Francia), e piú tardi il Laurent, il Vacherot, il De Ferron, il Franck, - Cournot e moltissimi altri: lesse e ammirò il Vico Auguste Comte, che nel 1844 ne scriveva allo Stuart Mill, e perfino Leone Gambetta ideava, da giovane, una storia generale del commercio condotta sullo schema vichiano dei « ricorsi »! La popolarità ottenuta per qualche tempo in Francia dal nome del Vico fu tanta che vi si accenna scherzosamente in piú luoghi dei romanzi del Balzac e nel Bouvard et Pécuchet del Flaubert. Ma l'efficacia di un pensiero di quella qualità non poteva essere né profonda né duratura nel tenace intellettualismo e spiritualismo francese. Il piú cospicuo risultato che essa raggiunse è forse da cercare nelle teorie del Fustel de Coulanges sulla città antica e sull'origine della feudalità.

Ma, per tornare all'Italia, se qui le aspirazioni al risorgimento nazionale, che portavano a rivendicare e celebrare tutte le glorie italiane, innalzarono il nome del Vico collocandolo quasi a lato di quello di Dante, il simultaneo risorgimento filosofico, nel districarsi dal sensismo e materialismo del secolo decimottaro, non poteva non fare capo all'ultimo grande filosofo idealista italiano e giovarsi del suo pensiero e valersi della sua autorità. Del Vico si raccolsero allora le opere complete e si moltiplicarono le edizioni dei singoli trattati. E poiché nel moto politico del Risorgimento si seguirono e in parte s'intrecciarono due correnti, la neoguelfa e la radicale; e il simile accadde in quello filosofico con le due tendenze idealịstico-cattolica e ideá- 
listico-razionale, rosminiano-giobertiana e bruniano-hegeliana; il Vico, eattolico e insieme libero filosofo, si prestava assai bene, com'è facile intendere, alle opposte simpatie e alle opposte interpetrazioni delle due scuole. Si vennero cosi formando di lui due immagini diverse, entrambe storicamente giustificate, benché una lo ritraesse piuttosto quale egli volle essere e l'altra quale effettualmente fu. Il Vico dei cattolici liberali era soprattutto il Vico dei punti metafisici, il platonico, il mistico del Dio inconoseibile, il tradizionalista dei prologhi al Divitto universale, e perciò anche filosofo schiettamente italiano da contrapporre a quelli della restante Europa, figli della Riforma; il Vico dei razionalisti, invece, l'audace ed eretico scopritore della Scienea nuova, e pereio filosofo europeo da mettere nella compagnia di Cartesio e Spinoza, di Kant e di Hegel. La prima immagine si può vedere nei libri del Rosmini, del Gioberti, del Tommaseo e di altri molti, tra $i$ quali non bisogna dimenticare quelli di un nobile scrittore napoletano, Enrico Cenni, che, meglio forse di tutti, amorosamente dipinse il Vico dei cattolici. La seconda immagine si ritrova presso $i$ filosofi e eritici, che dal 1840 in poi si educarono alla scuola dell'idealismo germanico; e segnatamente in Bertrando Spaventa e in Francesco de Sanctis, che cominciarono a vedere con chiarezza le relazioni tra il Vico e il pensiero europeo anteriore e posteriore, e a convertire $i$ semplici accenni e le vaghe impressioni di altri su tal proposito in interpetrazioni scientifiche e in giudizî determinati. E che gl'interpetri e critici del secondo indirizzo cogliessero nel segno e quelli cattolico-liberali o cattolicoidealisti si soffermassero in una posizione insostenibile, riproducendo nella loro irresolutezza e incoerenza l'irresolutezza e l'incocrenza medesima del Vico, era provato, tra l'altro, dalla diffidenza e ostilita che cattolici meno liberali ma piú coerenti (come lo spagnuolo Giacomo Bal- 
mes) seguitarono a dimostrare tenacemente verso l'autore della Scienza mova.

Non piccola efficacia esercitó il Vico nella storiografia italiana del medesimo periodo, concorrendo a formare un piú verace senso storico e spesso rischiarando le questioni che si dibatterano, come quella della condizione delle popolazioni romane sotto i Longobardi, a proposito della quale il Manzoni richiamò la vichiana * libertà signorile * ${ }^{1}$. E il suo pensiero dominò gli studî di giurisprudenza, specialmente nell'Italia meridionale; e, sebbene non producesse in questo campo grandi frutti scientifici, conferi a quei giuristi un'elevatezza e larghezza di criterî e una concretezza di giudizî, che rimasero dipoi un ricordo e un desiderio.

Dopo il 1870 , con la decadenza della filosofia in Italia e fuori, lo studio del Vico decadde; e per oltre un quarantennio non si sentí neppure il bisogno di ristampare le opere di lui. La monografia del Cantoni, che è del 186 nonostante alcune parti pregevoli, mostra già chiarissimi i segni della decadenza, perché tutta fondata sul concetto clie il Vico è tanto migliore quanto meno è metafisico e piú psicologo e storico; né ciò è detto in relazione all' intrinseca debolezza che il Cantoni gli addebita in cose cli filosofia, ma pel sottinteso convincimento, che è nel critico, della ranità di ogni metafisica, buona a suscitare entusiasmi solo nelle teste immaginose e confusionarie degli italiani del mezzogiorno. Al grande idealista della Scienza nuova fu riserbato perfino l'obbrobrio degli omaggi dei positivisti; i quali, nella loro meravigliosa ignoranza che è quasi innocenza, non clubitavano e non dubitano di allegare a conferma della loro professione di fede metodica il detto:

1 Si veda sul largo influsso vichiano in quel periodo la mia Storia della storiografia italiana nel secolo $X I T$ (Bari, 1921): efr. I'indice dei nomi. 
*verum ipsum factum »; e cioè, a senso loro, il vero è il fatto, il fatto bruto, che si vede e si tocca o che si crede di vedere e toccare. Scarsi gli scritti che recarono qualche serio contributo alla conoscenza di punti particolari delle sue dottrine. L'interesse pel Vico si è ravvivato solamente nell'ultimo decennio col generale ravvivarsi degli studî filosofici.

I due migliori lavori generali intorno al Vico, comparsi sullo scorcio del secolo passato, si debbono al cattolico tedesco Carlo Werner (1881), che ne espose con grande diligenza le dottrine filosofiche e storiche, giudicandole col criterio del teismo speculativo (svoltosi sotto l'influsso del Baader e della seconda filosofia schellinghiana), il quale è senza dubbio assai piú favorevole dello psicologismo del Cantoni alla comprensione del Vico; - e all'inglese Roberto Flint (1884), che serisse per la raccolta dei Philosophical classics una breve monografia sullo stesso argomento, esatta nei particolari e, se non profonda, guidata da limpido buon senso. E di recente, mentre il Sorel in Francia ha mostrato la fecondita di alcune dottrine del Vico e specialmente di quella dei ricorsi, tentandone applicazioni alla storia del cristianesimo primitivo e alla teoria del movimento proletario odierno, in Germania dal Biese e dal Mauther ne sono stati richiamati in onore i concetti sulla metafora e sul linguaggio.

Nondimeno, il Vico non ha ottenuto il posto che gli spetta nei libri dedicati alla storia della filosofia moderna; nei quali - o sia quello dello Höffding o l'altro, che gli ¿ tanto superiore, del Windelband, o qualunque altro si voglia, - il filosofo italiano, quando non sia passato del tutto sotto silenzio, appena viene rieordato come colui che avrebbe, dopo il Bossuet e prima dello Herder, tentato la dubbia scienza della * Filosofia della storia . Questa poca considerazione nasce in parte dalla scarsa conoscenza di 
quel che fu realmente il Vico, la cui molteplice energia di gnoseologo, cli teorico dell'etica, dell'estetica, del diritto, della religione rimane come seppellita sotto quel nome di « filosofo della storia ». Ma, per un'altra parte, è effetto del contraccolpo che la storia della filosotia risente dalla storia politica e dalla storia della cultura; onde i pensatori, la cui fortuna sociale seguí quella non felice dei popoli e degli stati ai quali appartennero, o che per altre cause e incidenti non operarono abbastanza largamente nella civiltà europea, sono sacrificati ad altri, di gran lunga meno importanti sotto l'aspetto filosofico, ma piú efficaci o piú noti come esponenti di vita sociale e rappresentanti d'indirizzi di cultura; e dove si terrebbe impossibile ignorare, a mo' d'esempio, il Paley o il D'Holbach 0 il Mendelssohn, sembra naturale ignorare un Giambattista Vico, che pure è, in mezzo a costoro, gigante tra pigmei. Il che quanto sia storicamente ingiusto è dimostrato, in tesi teorica, dalla distinzione, su cui insistiamo, tra storia della filosofia e storia della cultura; e, nel caso particolare del Vico, da tutto il nostro lavoro, dal quale appare evidente la lacuna che la trascuranza dell'opera di lui lascia aperta nella storia generale del pensiero europeo ai principî del secolo decimottavo. 


\section{III}

\section{CenNi Bibliografici}

\section{I}

Opere del Vico

Il piú antico scritto del Vico, che ci sia pervenuto, è la canzone Affetti di un disperato, composta di certo prima che l'autore raggiungesse il venticinquesimo anno, a Vatolla nel Cilento (dove si trattenne per eirca un novennio eome pedagogo in easa Rocca), e stampata con la lata di Venezia, Gonzatti, 1693. Seguirono versi e orazioni di carattere quasi meramente rettorico.

Il carattere filosofico si accentua nelle sei orazioni che il Vico lesse nell'universita di Napoli dal $1699^{\circ}$ al 1707 e che, non messe a stampa da lui, furono poi ritrovate e pubblicate dal Galasso (Napoli, Morano, 1869). In queste orazioni, sebbene si mostrino alcune tendenze della sua mente, c'è ancora la filosotia tradizionale, non senza qualehe traecia di cartesianismo. L'opposizione al cartesianismo, e insieme la professione metodica del Vico, si affermano per la prima volta nell'orazione per la solenne inaugurazione degli studî dell'anno 1708, intitolata: De nostri temporis studiorum ratione, pubblicata dall'autore stesso 
l'anno dopo (Napoli, Mosca, 1709). Un lungo escurso (\$\$ 1215) contiene uno schizzo della storia della giurisprudenza romana, primo saggio degli studî storici che condussero piú tardi al Diritto universale e alle due Scienze nuove.

L'anno seguente apparve la prima costruzione filosofica e storica del Vico: il De antiquissima italorum sapientia ex lingua latince originibus eruenda, o meglio il solo primo libro (Napoli, Mosca, 1710); gli altri due non furono mai pubblicati, ma di quel che dovessero contenere può dare idea ciò che se ne dice nell'Autobiografia. Oltre la gnoseologia (nella prima forma) e la metafisica del Vico (da lui mantenuta poi sempre salda), il De antiquissima tenta di ritrarre per la prima volta la sapienza primitiva o un caso particolare di quella sapienza (l'italica); quantunque, come si è gia detto nel testo del nostro lavoro, il tentativo sia fondato sull' idea clie quella sapienza fosse filosofica, e condotto col criterio della trasmissione delle civiltà dal Vieo poi rifiutato, come rifiutò l'opinione tradizionale, ivi ancora accolta, dell'origine ateniese della legge delle dodici tavole. Inaccettabile è dunque il gindizio del Cantonı (G. B. Vico, p. 38) che il De antiquissima formi « una strana anomalia nella storia del pensiero del Vico, essendo contrario a tutta la sua vita scientifica, alle sue tendẻnze, ai prineipî e al metodo che quasi universalmente applicò poi nelle sue ricerche storiche \#; ed è vero l'opposto, che esso è il primo anello del futuro svolgimento, senza del quale i impossibile intendere il pensiero posteriore. - Le censure, che il Giornale de' letterati d'Italia (1711, tomi V e VIII) mosse cosi alla tesi storica come ad alcune delle tesi filosofiche del $D e$ antiquissima, dettero origine a due importanti Risposte del Vico (Napoli, Mosca, 1711 e 1712), nelle quali le sue idee gnoseologiche e metafisiche sono difese e schiarite. Alla parte non piú messa in istampa del De antiquissima si riannodavano le meditazioni di filosofia della medicina, dalle 
quali il Vico trasse un opuscolo: De axquilibrio corporis animantis, che molti anni dipoi pensava di pubblicare e che è andato perduto; onde di quelle, come delle sue speculazioni di fisica (che dovevano costituire il Liber physicus), non si sa altro se non ciò che egli stesso dice nell'autobiografia.

Tralasciando gli scritti rettorici e per commissione, dei quali il piú esteso è il De rebus gestis Antonii Caraphcei (Napoli, Mosca, 1716), i nuovi frutti del suo pensiero (che si andò concentrando sui problemi morali e storici), prima accennati in una prolusione del 1719 della quale il sommario è nell'autobiografia, furono condensati dal Vico, in italiano, nel 1720 , in un programma a stanpa di quattro pagine fitte a due colonne, noto sotto il nome di Sinopsi del diritto niversale, e svolti nell'ampia trattazione: De universi iuris uno principio et fine uno liber unus (Napoli, Mosca, 1720), compiuta l'anno dopo dal Liber alter qui est de constantia iurisprudentis, e accresciuta nel 1722 dalle Notce in duos libros, ecc., che rappresentano un ulteriore avanzamento (ivi); la quale opera si suole designare nel suo complesso, seguendo l'esempio dello stesso autore, col nome di Divitto universale.

Questo libro, secondo il Cantoni (op. cit., p. 243), rappresentercbbe il culmine dell'attività scientifica del Vico: giudizio non meno inaccettabile del precedente. L'autore (Opp., V, 10-11) rifiutò il Diritto universale, perché gli pareva che vi perdurassero il pregiudizio e la pretesa di "scendere » dalla mente di Platone e degli altri filosofi a quelle degli nomini primitivi, onde in esso avrebbe errato "in alquante materie ; ma lo disse anche a ragione, " abbozzo della Scienza nuova », qual è veramente. Le idee sulla poesia vi sono ancora perplesse, Omero non vi è ancora un mito, i canoni mitologici sono meno unitarî di quel che divennero poi, per l'origine delle XII tavole si affaccia 
un'ipotesi ibrida, la teoria dei ricorsi vi è appena debolmente adombrata, e insomma cosí la storia ideale eterna come la gnoseologia, sulla quale essa si fonda, sono ancora immature. L'opera è rifusa nelle posteriori, salvo ciò che riguarda la generale filosofia etica e giuridica (che non è molto originale) e salvo alcuni svolgimenti storici che nelle opere posteriori ricompaiono solo in accenno.

$\dot{E}$ andato perduto il manoscritto di un'opera italiana, divisa in due libri, in cui il Vico esponeva le sue dottrine «per via negativa », ossia con metodo prevalentemente polemico. In modo positivo, invece, e in forma concisa, le espose nei Princip̂́ di una Scicnza nuova intorno alla comune natura delle nazioni, per la quale si ritrovano $i$ principî di altro sistema del diritto naturale delle genti (Napoli, Mosca, 1725), che sono conosciuti con la denominazione (anche questa proveniente dall'autore medesimo) di Prima scienza nuova.

Nello stesso anno in cui pubblicò la Prima scienza nuova, cioè nel 1725, il Vico narrò la storia dei suoi studî: Vita di G. B. V. scritta da sé medesimo, che fu inserita nella Raccolta di opuscoli scientifici e filologici del Calogerà (Venezia, Zani, 1728, vol. I, pp. 145-256). Dei minori scritti di questo periodo sono notevoli altresi lo due orazioni in morte della contessa di Althann (1724) e della marchesana della Petrella Angiola Cimini (1727); it volumetto Vici vindicia (Napoli, Mosca, 1729), contenente una difesa di carattere personale (con un'importante digressione teorica sul «riso s) contro una maligna noterella inserita negli Acta lipsiensia del 1727 intorno alla Scienza nuova; e alcune lettere bellissime al Giacchi, al Degli Angioli, all'Esperti, al De Vitry e al Solla, sul contrasto tra la sua opera e le condizioni degli studî a quel tempo.

Alla Prima scienza nuova il Vico pensò di aggiungere una lunga serie di Anmotazioni (effettivamente poi scritte 
ma andate disperse) in una ristampa che se ne preparava a Venezia tra il 1728 e il 1730. Ma poiché questil non ebbe piú effetto e, d'altro canto, quel libro non lo soddisfaccva se non proprio per le materie (egli dice), per l'ordine tenuto $(O p p ., \mathrm{V}, 11)$, si risolse al dare un'esposizione affatto nuova delle sue dottrine nei Cinque libri de' principî di una Scienza nuova d'intorno alla comune natura delle nazioni, in questa seconda impressione con piu propia maniera condotti e di molto accresciuti (Napoli, Mosca, 1730), che formano la Seconda scienza nuova. Quantunque il Cantoni (op. cit., pp. 238-9) consideri quest'opera come una senile degenerazione del pensiero del Vico, essa è invece il risultato necessario e la forma perfetta a cui mettono capo i tentativi precedenti; ed è il libro che, insieme col De antiquissima e con l'autobiografia, basta a fornire tutto l'essenziale per la conoscenza del pensiero di lui. Nel Diritto universale e nella Prima scicnza nuova si può spigolare soltanto qualche particolare dipoi tralasciato; ma, pel resto, vi compaiono le medesime dottrine della Seconda scienza nuova in un modo meno profondo e meno sicuro, e, certamente, meno vichiano. Il confronto particolare tra queste tre opcre fu eseguito con diligenza nei sommarietti apposti dal Ferrari alle sue edizioni della Prima e della Seconda scienza nuova; e moltissimi altri riscontri e piú particolareggiati possono vedersi ora nella edizione della Scienza nuova, curata dal Nicolini.

Anche alla redazione del 1730 il V., senza quasi piú mutarue l'ordine e la sostanza, andò facendo, negli anni tra il 1731 e il 1736 circa, molte rariazioni c aggiunte, che poi incorporò per gran parte nel testo in un manoseritto definitivo, sul quale fu condotta l'edizione dei Principî di una Scienza nuova d'intorno alla comune natura delle nazioni, uscita sei mesi dopo la morte del V. (Napoli, nella stamperia muziana, 1744). Sono serbati nella 
Biblioteca nazionale di Napoli gli autografi cosi di questo manoseritto come di due altri anteriori di aggiunte e correzioni, dai quali trassero alcuni brani rimasti inediti il Giordano (Napoli, 1818) e il Del Giudice (Napoli, 1862), e ora tutti i brani inediti e le varianti ha estratto il Nicolini per la sua edizione.

Dopo la Seconda scienza nuova, il Vico scrisse pochissime cose: notevoli, tra esse, l'orazione De mente heroica (Napoli, 1732), l'aggiunta all'autobiografia (1731) e alcuni sonetti, nei quali, sebbene composti (come quasi tutti i stoi versi) per occasione e commissione, risuona, qua e là, uma nota personale.

Ristampe, raccolte e tradezioni

Degli scritti minori del Vico si fecero due raccolte, una delle sole Latina orationcs, a cura di F. Daniele (Napoli, 1766), e l'altra, ricca di cose inedite ma non esente da raffazzonature dell'editore, degli Opuscoli italiani e latini, in quattro volumi, a cura di C. A. de Rosa marchese di Villarosa (Napoli, 1818-1823). Il Villarosa ebbe tutto ciò che avanzava delle carte del Vico dal figliuolo di costui, Gennaro; e i preziosi autografi si serbano ancora a Napoli in casa dei miei cari amici ingegneri Tommaso e Vincenzo. de Rosa di Villarosa.

Delle Opere complete la prima, e si può dire unica edizione perché riprodotta in tutte le altre, è quella di Giuseppe Ferrari in sei volumi (Milano, Classici italiani, 1835-7), listampata con qualche migliormento nel 1852-54. Le opere a cura di N. M. Corcia (Napoli, tipografia della Sibilla, 1834, due voll.), sono, invece, una scelta; e le Opere a cura di F. Predari (Milano, Bravetta, 1835) si arrestano al primo e disordinato volume. Incompleta e disordinata è 
anche l'edizione di Napoli, Iovene, 1840-41, che segue l'edizione del Ferrari, ma pur contiene qualche bazzecola inedita. Materialmente condotta sulla ferririana, c poco corretta, è l'edizione napoletana delle Opere (i primi volumi presso la tipografia dei Classici italiani, o gli altri presso l'editore Morano) in otto volumi (I-II, 1858, III, 1861, IV, 1859, V-VI, 1860, VII, 1865, VIII, 1869); la quale, per altro, ̀̀ la piú completa di tutte, essendovi unite la Sinopsi, le Istituzioni oratorie e le Mrazioni latine edite dal Galasso (che vennero fuori dopo l'edizione Ferrari); vi sono aggiunte anche versioni italiane del De ratione, del De antiquissima e del Diritto universale, a cura dell'avv. F. S. Pomodoro.

Scritti inediti o sparsi del V., non compresi in nessuna di tali edizioni, sono raccolti nel Croce, Bibliografia vichianu e Primo e Secondo supplemento, e Nuove ricerche, Ş 2; e in un opuscolo di B. Dovati : si veda piú oltre.

Una edizione critica della Seconda scienza nuova è stata pubblicata nella Collana dei classici della filosofia moderna rivetta da B. Croce e G. Gentile (Bari, Laterza, 1911-16, voll. 3). É dovuta al d." Fausto Nicolini, che si è valso per essa degli autografi ed ha arricchito l'edizione Ferrari, che conteneva solo i brani soppressi di quella del 1730, di tutti i brani delle redazioni intermedie fino al testo del 1744; ha, inoltre, riscontrato le citazioni vichiane e recato in nota i luoghi degli autori classici e moderni ai quali si riferiva il V.; additati i molti errori d'erudizione, procurando sempre che fosse possibile di mostrarne la genesi; schiariti i punti oscuri col riferimento alle altre opere del Vico; e, finalmente, ha riformato (secondo un desiderio piú volte espresso anche da autorevoli letterati come il Tommaseo) l'ortogratia e la punteggratura. Dell'edizione ferrariana sono riprodotti in questa del Nicolini, ma alquanto ritoccati, gli utili sommarietti: In un'ampia iutroduzione si studia il Vico scrittore e si da notizia delle suc- 
cessive redazioni e rimanipolazioni della Scienza nuova; due escursi mostrano come il Vico giunse via via alla sua teoria omerica e all'altra analoga sulla Legge delle XII Tavole; e le ricerche nei tre volumi sono agevolate da un minuto indice analitico.

Lo stesso Nicolini, col Croce e col Gentile, attende a una nuova edizionc delle opere complete, che farà parte della raccolta degli Scrittori d'Italia del Laterza e il cui disegno e indice particolareggiato si puỏ leggere nel Croce, Secondo supplemento alla Bibliografia vichiana (pp. 102-113). Di questa edizione sono stati pubblicati il vol. I (Le orazioni inaugurali, il De italorum sapientia e le polemiche, a cura del Gentile e del Nicolini, 1914), e il vol. V (L'autobiografia, il carteggio e le poesie varie, a cura del Croce, 1911).

Le opcre latine del V. sono state piú volte tradotte in italiano: il De antiquissima da un anonimo, che forse fu Vincenzo Monti (1816), e poi dal Sarchi (1870); il primo libro del Diritto universale dal Corcia (1839), dall'Amante (1841), dal Giani (1855) e dal Sarchi (1866), e tutti i due libri, nonché il De ratione e il De antiquissima, come si è già detto, dal Pomodoro. La Seconda scienza muova fu tradotta in francese, ma molto abbreviata, da Giulio Michelet (col titolo: Principes de la philosophie de l'histoire, Paris, Renouard, 1827, e piú rolte ristampata), e di nuovo, completa, da un anonimo che si designa come "l'auteur de l'Essai sur la formation du dogme catholique » e che fu la principessa di Belgioioso Cristina Trivulzi (Paris, Renouard, 1844). Completa anche, c fornita di ottime note, è la traduzione tedesca di W. E. Weber (Leipzig, Brockhaus, 1822), che suggerimenti e aiuti ebbe da Gaspare Orelli. In inglesc, si ha solo la versione del libro su Omero, condotta sulla francesc del Michelet e inserita nell'opera di H. Nelson Coleridge, Introduction to the study of the greek classic poets $\left(3 .^{a}\right.$ ediz., London, Murray, 
1846). Il Michelet tradusse alcune delle operette minori del V., che si accompagnamo alla Scienza mova nell'edizione Quvres choisies de V. (Paris, Hachette, 1835, e in ristampe). Del primo libro del Diritto universale si ha un compendio in tedesco di K. H. Mïller, primo rolumetto di una serie non proseguita di Kleine Schriften del V. (Neubrandeburg, Brünslow, 1854).

A supplemento dell'autobiografia, il Villarosa raccolse le notizie degli ultimi anni della vita del Vico, e le mise come continuazione di quello seritto nella sua edizione degli Opuscoli, rol. I (1818).

Questo supplemento, e tutto ciò che di poi è venuto fuori di documenti o di ricordi di contemporanei intorno al V., si trovano liaccolti nel quinto volume della nnova edizione, annunziata di sopra (p. 303), delle opere del V., intitolato: L'autobiografia, il carteggio e le poesie varie, a cura di B. Croce (Bari, Laterza, 1911). Posteriormente, alcune aggiunte, in CROCE, Nuove ricerche, $\$ 1$, e Nuove curiosità storiche (Napoli, Riceiardi, 1922), pp. 12952, e nel volumetto del Doxatr.

\section{IV}

Lemteratura intorno at. Vico

Le tre sole monogratie intorno al Vico, che possano ancora essere lette con frutto (quella del Ferrari, pur cosi benemerito editore, La mente del $T$., è degna di essere pietosamente (limenticata), sono: 
1. Carlo Cantoni, G. B. V., studî critici e comparativi (Torino, Civelli, 1867). Cfr. per alcune riserve A. FAgGI, in Rivista filosofica italiana, vol. IX, 1906, pp. 593-606, e G. Gentile, in Critica, vol. V, 1907, pp. 197-201.

2. KarL Werner, G. B. T. als Philosoph und gelehrter Forscher (Wien, Braumüller, 1881). Cfr. Zeitschrift für Philosophie und philos. Kritik, vol. LXXII, 1883, pp. 139-152.

3. Robert Flint, V. (Edinburgh a. London, 1884). Traduzione italiana di F. Finocchictti (Firenze, 1888).

Si veda ciò che intorno a esse si è detto di sopra, pp. 326-7.

Dei lavori brevi di carattere generale hanno singolare pregio :

1. B. Spaventa, G. B.V., in Prolusione e introlluzione alle lezioni di filosofia (Napoli, Vitale, 1862), pp. 83-102: opera ristampata col titolo: La filosofia italiana nelle sue relazioni con la flosofia europea, a cura di G. Gentile (Bari, Laterza, 1908); si veda a pp. 111-135 di questa ristampa.

2. F. DE SANCTIS, Storia della letteratura italiana (Napoli, Morano, 1870; molte ristampe), vol. II, pp. 342-362.

3. F. Fionentino, Lettere sopra la 'Scienza nuova' (Firenze, 1865); ristampate in Scritti varî (Napoli, Morano, 1871), pp. 161-211.

4. E. Cauer, G. B. V. und seine Stellung zur modernen Wissenschaft (nel Deutsches Museum, diretto da R. Prutz e W. Woelfsohn, Leipzig, Hinrichs, a. I, 1851, vol. I, pp. 249-265).

Per la trattazione piú o meno larga di parti speciali sono da tenere presenti :

1. F. A. Wolf, G. B. V. ïber den Homer (nel Museum der Alterthumsuissenschaft, Berlino, 1807, vol. II, pp. 555$570)$.

2. J. K. von Orelc1, V. und Niebuhr (nello Schweizerisches Museum di Aarau, vol. I, p. 184 segg.). 
3. C. IANandu, Sulla natura e necessiti della scienza delle cose e delle storie umane (Napoli, Porcelli, 1818, e Milano, Fontana, 1832).

4. EMrerico AMrari, Critica di una scienza della legislazione comparata (Genova, Istituto dei sordomuti, 1857). Cfr. intorno a questo libro K. Werner, $E$. A. in seinem Verhaltniss zu G. B. V. (Wien, 1880; dai Sitzungsberichte der phil.-histor. Classe della Accalemia imperiale di Vienna, vol. XCVI).

5. F. Acrl, Teoria del $V$. intorno alle idee o paradimmi (in Abbozzo di una tenria delle idee, Palermo, Lao, 1870; e con modificazioni nel volume: Videbimus in anigmate, Bologna, Mareggiani, 1907, pp. 287-313).

6. E. Cenvı, esposizione della metafisica del V., a pp. 10918.2 del volume nel quale nessuno la cercherebbe, perché il titolo suona: Considerazioni sull' Italia ad occasione del traforo del Gottardo (Firenze, Cellini, 1884).

7. E. Bouvy, De V. Cartesii adversario (Paris, Hachette, 1889).

8. E. Bouvy, La critique dantesque an dix-huitieme siecle: Dante et $V$. (Paris, Leroux, 1892).

9. G. Sorec, Étude sur V. (nel Devenir social, di Parigi, vol. II, 1896); e si veda, altresi, dello stesso autore: Le systeme historique de Renan (Paris, Jacques, 1905), passim.

10. B. Labanca, G. B. V. e i suni critici cattolici (Napoli, Pierro, 1898).

11. G. Rossi, V. nei tempi di V. (nella Rivista filosoficr ifaliana, rol. II, 1899 , pp. 294-319, e seconda parte, ivi, vol. $\mathrm{X}, 1907, \mathrm{pp}$. 602-634).

12. G. Madgan, Étude sur l'évolution intellectuelle de l'Italie de 1657 à 1750 environ (Pariss, Hachette, 1909).

13. G. Finsler, Homer in der Neuzeit von Dante bis Goethe (Leipzig, Tcubner, 191으). 
14. G. Gextule, Studi vichirni (Messina, Principato, 1915). Contiene, tra l'altro, un'importante monografia su Lo srolgimento della filosofia di G. B. V., pp. 15-143.

15. F. Nicolini, Ferdinando Galiani e G. B. V., in Giorn. stor. d. lett. ital., 1918, vol. LXXI.

16. Id., Divagazioni omeriche (Firenze, Ariani, 1919).

17. O. v. Gemmingex, Vico, Hamann und Herder, Inaugural-dissertation (Bona-Leipzig, Noske, 1918).

18. A. Scrocca, G. B. V. nella critica di B. Croce (Napoli, Giannini, s. a., ma 1919), dal punto di vista cattolico.

19. Benvenuto Doxati, Autografi e documenti vichiani inediti o dispersi, note per la storia del pensiero del $\mathrm{V}$. (Bologna, Zanichelli, 1921).

20. Circa i miei lavori precedenti sul V., si avverta che la materia del capitolo sulla dottrina estetica vichiana, B. Croce, Estetica ${ }^{5}$ (Bari, Laterza, 1922), cap. V, pp. 249265, è rielaborata in forma piú matura nel capitolo IV della presente monografia; lo scritto sull'Etica del V. (in Critica, VI, 1908, pp. 71-77) è rifuso nei capp. VI-VIII; e cosí quello sui Lineamenti di storia letteraria in G. B. V. (ivi, pp. 460-80), nei capp. XVI e XVIII; gli altri scritti sparsi hanno, in genere, interesse solamente erudito, filologico o polemico. -- Posteriormente alla prima ed. di questo libro, ho pubblicato: 1. Le fonti della gnoseologia vichiand (in Atti d. Acc. Pontan., vol. XLII; ristamp. nel vol. Saggio sullo Hegel e altri scritti di storia della filosofia, Bari, 1913); 2. La dottrina del riso e dell'ironia in G. B. T., ristamp., ivi; 3. Il Vico e la critica omerica, ristamp., ivi; 4. Francesco Bianchini e G. B. Vico, ristamp. in Conversazioni critiche (Bari, 1918), II, 101-109; 5. Il Vico e Gius. Ferrari; ivi, II, 124-30. Dell'influsso del V. sugli studî italiani nel corso del secolo decimonono ho trattato ampiamente nella Storia della storiografia italiana nel secolo decimonono (Bari, 
1921). Sulla posizione del V. nella storia della critica dantesca, v. La poesia di Dante (30. ${ }^{2}$ ed., Bari, 1922), pp. 173-4, 179-80.

Del resto, tutta la letteratura vichiana (con estratti dei libri, opuscoli e articoli piú rari e con documenti inediti), come tutte le piú minute notizie sulle edizioni degli scritti del V., si trovano raccolte nelle tre memorie, alle quali piú volte si ì fitto riferimento: B. Croce, Bibliografia vichiana contenente nella parte I il catalogo delle edizioni, traduzioni e manosivitti delle opere di G. B. V.; nella parte II, quello dei giudizi e lavori storico-critici intorno al V. sino all'anno corrente; nella parte III, lettere inedite del V. e al V., documenti e altri scritti inediti o rari, e e $a$ ric appendici illustrative (Napoli, 1904; estratto dagli Atti dell'siccudemia pontuniana, di Napoli, vol. XXXIV, di pp. xı-127, in 4..$^{\circ}$ ); Supplemonto alla Bibliografia vichiana (ivi, 1907; estr. dagli Atti cit., vol. XXXVII, di pp. 34, in $4 .^{\circ}$ ), e Secondo supplemento (ivi, 1911, estr. dagli Atti cit., vol. XL, di pp. 116 , in $\left.4 .^{\circ}\right)$; - riunite anche tutte e tre in un sol volume col titolo: Bibliografia vichiana, raccolte di tre memorie presentate all'Accademia pontaniana di Napoli nel 1903, 1907 e 1910, con appendice di F. Nicolini (Bari, Laterza, 1911). Continuazione di queste memorie sono le Nuove ricerche sulla rita e le opere del $V$. e sul vichismo, in Critica, voll. XV-XIX, 1917-21. Si veda anche Per la biografia di G. B. V., ivi, XIX, pp. 871-87 (e ora in Nuove curiosità storiche, Napoli, Ricciardi, 192.2, pp. $123-52)$. 


\section{INDICE DEI NOMI}

\section{A}

Abrano, 152.

Achille, 102, 130, 191, 192-4, 195, $199,233$.

Acilio Glabrione, 203.

Acquaviva M., 143.

Acri F., 339.

Adamo, 53.

Adriano, 222.

Agamennone, 178, 191, 192, 193.

Agide, 176.

Agostino (s.), 169.

Alcinoo, 192, 195.

Alessandro, 94, 130, 137, 221.

Amante E., 336.

Amari E., 339.

Anacreonte, 202.

Anfitrione (iscrizione di), 196.

Angelis (de) A., 322.

Angelis (de) Gh., 305, 332.

Annibale, 165.

Anteo, 187.

Apollo, 102, 168, 187.

Archiloco, 185.

Ariosto, 233.

Aristarco, 195.

Aristide, 92, 130, 204.

A ristotele. 15, 25, 35, 48, 51, 53, $70,94,97,145,175,182,202$, 298.

Arnaud, 241.

Ascalona (duea d'), 290.

Atlante, 164.
Attico Tito Pomponio, 89.

Attilio Regolo, 176.

Aubignac (d'), 198, 199.

Angusto, 204, 219-20.

\section{B}

Baader, 323, 327.

Bachhofen, 256.

Bacone F., 15, 25, 33, 35, 64, 76, $115,162,163$.

Ballanche, 322, 324.

Balmes, 325 .

Balzac O., 324.

Balzo (del) A., 241.

Bandiera G. N., 302.

Banier, 64, 71.

Bartoli D., 132.

Bartolo da Sassoferrato, 231.

Baumgarten, 48.

Bayle P., 80, 89, 161, 246.

Boroso, 164.

Bianchini F., 340.

Biese A., 327.

Bignon (du), 317.

Boccaccio, 63, 136, 171, 234.

Bochart S., 61, 208.

Böckh A., 323 .

Bodin, 174, 228.

Boiardo, 283.

Bonamy, 316.

Bossuet, 120, 154, 32 7 .

Boulanger, 317, 320 . 
Bouvy E, 339.

Boyle R., 143.

Brenno, 80, 82 .

Briseide, 191, 193.

Bruno Giordano, 288.

Bruto Giunio, 176, 179, 209, 210.

\section{C}

Caligola, 130.

Calipso, 195.

Calogerà, 332.

Campanella T., 96, 98, 128, 288.

Cantoni C., 326, 327, 330, 331, 333, 337.

Capasso N., 299, 302.

Capece G., 290, 291.

Capeto (Ugo), 228-29.

Caracalla, 221.

Cardano, 4.

Carlo di Borbone, 289.

Carneade, 80, 8t.

Cartesio, 1-3, 5, 6, 7, 8, 9, 10, 12, $14,15,18,19,26,27,32,81$, $142,144,244,251,298,325$.

Castelvetro, 53.

Cauer E., 338.

Cenni E., 325, 339.

Cerere, 167.

Cesare, 180, 137.

Cesarotti, 317, 318.

Chastellux, 316.

Chateaubriand, 171, 324.

Cherea, 168.

Cibele, 187.

Cicerone, 75, 89, 163, 184, 202, $212,244$.

Ciclici (poeti), 200.

Cimini Angela, 293.

Cincio Alimento, 209.

Circe, 195.

Cirillo N., 302.

Cola di Rienzo, 233.

Colangelo F., 320.

Comte A., 324.

Concina N., 305, 306.

Confucio, 187.

Conti A., 306, 317.

Conti N., 63.

Corcia N. M., 334, 336.
Corneille, 244.

Cornewal Lewis, 256.

Corrado III, imperatore, 232.

Costantino, 221.

Cournot, 324 .

Consin, 324.

Criseide, 191.

Cristofaro (di) G., 286.

Croce B., 335, 336, 337, 340-1.

Cuiacio, 226, 227.

Cuoco V., 135, 321, 32.2.

Curiazî, 182.

Curio, 176.

Curzio, 176.

\section{D}

Dafne, 168.

Dale (van) A., 71 .

Daniele F., 334 .

Dante, 155, 156, 234-6, 256, 299, $306,307,324,341$.

Daun (conte di), 290.

Decio, 176.

Degélando, 322.

Deschamps S., 93.

Diana, 167, 168, 177, 187, 191.

Diodoro, 163, 212.

Diomede, 191.

Dione siracusano, 212, 237.

Dionigi d'Alicarnasso, 211, 212.

Domiziano, 130.

Donati B., 335, 337, 340.

Dracone, 184, 188.

Dubois (cardin.), 245.

Dugald Stewart, 251.

Duni, 316.

\section{F}

Ecateo milesio, 197.

Elena, 193.

Eliano, 184.

Elingio Ingevaldo, 53.

Endimione, 168.

Enea, 208.

Ennio, 203.

Epicurei, 99.

Epicuro, 84, 103, 142. 
Eraclito, 212.

Ercole, 66, 67, 168, 187.

Erodoto, 75, 166, 195, 197.

Ermodoro, 212, 213.

Esiodo, 201.

Esopo, 70, 187, 201.

Esperti, 332.

Esteban F., 304, 305.

Ettore, 192, 193.

Europa, 187.

Eusebio, 184.

Ezechiele, 94.

\section{F}

labio Massimo, 214, 215.

Fabio Pittore, 209.

Fabrizio, 176.

Faggi A., 337.

Faramiondo, 171.

Ferrari G., 311, 333, :334, ¿137, 340.

Ferron (du), 324.

Fichte, 253.

Ficino M., 4, 143.

Filangieri, 319, 321.

Filippo V, 290.

Finetti, 302, 319, 323.

Finsler, 339.

Fiorentino F., 338.

Flaubert, 294, 324.

Flint R., 327, 338.

Fontenelle, 71, 161.

Foscolo, 178, 321, 322.

Fozio, 246.

Franck A., 324.

Fustel de Coulanges, 256, 324.

G

Gaeta (di) M., 303, 304.

Galasso A., 329.

Galeno, 51.

Galiani 1., 306, 317, 318.

Galileo, 10, 13, 15, 143, 146.

Gambetta L., 324 .

Ganimede, 168.

Garofalo P., 340.

Gassendi, 143.
Geminingen von O., 340.

Genovesi A., 291, 306.

Gentile G., 336, 338, 339.

Gerning, 321.

Giacchi B., 286, 287, 303-4, 332.

Giamblico, 51.

Giani C., 336.

Giannone P., 124, 289.

Giansenisti, 99.

Gimma, 303.

Giobbe, 184.

Gioberti, 325.

Giordano A., 334.

Giordano L., 173.

Giove, 55, 65, 69, 71, 88, 102, 120 , $152,167,168,169,186,187,188$, $189,191$.

Girolamo (san), 184.

Giudice (del) G., 334.

Giuljano Salvio, 222.

Giunone, 168, 186, 191.

Giuselpe ebreo, 95, 184.

Giustiniani L., 320 .

Giustiniano, 100, 220.

Giustino, 177.

Goetho, vin, 319.

Gorgia, 185.

Gracchi, 216.

Grandi, 316.

Gravina, 31 .

Grimm, 256.

Gronovio, 163.

Grozio, 22, 31, 32, 47, 76, 77, 78, $84,89,90,91,94,95,99,100$, $103,108,163,236,287$.

Guglielmo pugliese, 233.

Guntero, 233.

\section{$\mathbf{H}$}

Hamann, 252, 319.

Hegel, 251, 252, 253, 254, 300, 317, 323,325 .

Herder, 150, 252, 319, 327.

Heyne, 252, 256, 318.

Hobbes, 80, 81, 89, 103, 253.

Höffling H., 327.

Hoffmann, 246.

Hoffmannswaldau, 236.

Holbach (d'), 328. 
Huet D. (Uezio), 64.

Humboldt, 252.

Hume D., 252.

\section{I}

Iannelli C., 286, 322, 338.

Idantura, 51.

Ifigenia, 178.

Ippocoonte (iscrizione d'), 196.

Iside, 184.

Issione, 187.

\section{J}

Jacobi, 251, 319, 323.

Jouffroy, 324.

\section{K}

Kant, 251, 252, 253, 313, 325.

\section{工}

Labanca B., 320, 339.

Lambert, 256.

Lami G., 319.

Laomedonte (iscrizione di), 196.

Lattanzio, 95.

Laurent, 324.

Laurenzano (duca di), 291.

Lazio W., 53.

Lebrun, 173.

Leclerc G. (Clericus), 64.

Leda, 168.

Legge canuleia, 214.

Legge delle dodici tavole, 211-2.

Legge petelia, 215.

Legge publilia, 215.

Legge regia di Triboniano, 219.

Leibniz, 31, 32, 61, 145, 146, 257.

Lelio, 92 .

Lerminier, 324.

Licambe, 185.

Licurgo, 184.

Lipsio G., 184.

Livio Andronico, 203.
Livio Tito, 165, 169, 211.

Locke, 80, 81, 83, 143, 253.

Lodovico (padre), 305.

Lohenstein, 236.

Lomonaco F., 321.

Longino, 196, 203.

Lucio Emilio Regillo, 203.

Lucrezio, 142, 203.

Luigi XIV, 291.

Luzán I., 306.

\section{M}

Macchia (congiura di), 290.

Machiavelli, 85, 80, 82, 103, 128, $210,223$.

Malebranche, 8, 32, 81, 145, 244.

Mallinkrot B., 53.

Manlio capitolino, 176.

Manlio l'imperioso, 176.

Manzoni, 321, 326.

Marino G. B., 236.

Marte, 168, 187, 191.

Marx C., 256.

Maffei S., 157.

Maugain G., 339.

Mauthner F., 327.

Mazzoni I., 143.

Medea, 235.

Menandro, 201.

Mendelssohn, 328.

Menelao, 182, 193.

Mercurî Trismegisti (i due), 164, 187.

Mercurio, 193.

Mercurio gotico, 53.

Merian, 318.

Merlino, 171.

Metastasio, 244, 302.

Michelet, 150, 322, 323, 324, 336.

Minosse, 184, 187.

Minotauro, 187.

Mommsen, 256, 323.

Montesquieu, 317.

Monti V., 321, 336.

Moreri, 246.

Mosco, 202.

Mosè, 95, 152, 204-5.

Müller K. O., 252, 256, 323, 337.

Muratori, 82, 157, 321. 


\section{$\mathbf{N}$}

Nelson Coleridge, 386.

Nerone, 130.

Nettuno, 167, 187.

Nevio, 203.

Newton, 146, 305.

Nicole P., 81.

Nicolini F., 15̃7, 299, 333, 334, $335,336,340,341$.

Niebuhr, 256, 323.

Nietzsche F., 256.

Nifo A., 143.

Noè, 152 .

Numa Pompilio, 94, 188, 195.

Oldendorpio, 226.

Olivieri A., 339.

Omero, 39, 65, 155, 156, 169, 19i$205,234-5,256,257,331$.

Orazî, 182, 201.

Orazio Flacco, 185, 204.

Orelli, 323, 336, 338 .

Orfeo, 16t, 187.

Origene, 184.

Orlando, 170, 233 .

\section{$\mathbf{P}$}

Pagano M., 317, 321.

Pais E., 256.

Pallavicino, 81 .

Paoli (padre), 302.

Paolo veneto, 24?.

Paride, 182.

Pascal, 81.

Patrizzi F., 53, 143.

Patroclo, 192.

Penelope, 195.

Perseo, 187.

Petrarea, 156, 234.

Piccolomini A., 143.

Pico della Mirandola, 14:.

Pindaro, 201, 204.

Pirro, 176, 208.

Pisistrato, 198.
Pitagora, 12, 94, 165, 188, 208, 212.

Pitagorici, 237.

Platone, $16,35,47,51,53,65,70$, 76, 103, 104, 108, 109, 121, 128, 164, 173, 188, 204, 208, 296, 331.

Planto, 3, 182.

Plinio, 213.

Plutarco, 91. 177, 195, 203, 210.

Polibio, 92, 128, 208, 210, 212.

Polissena, 194, 293.

Pomodoro F. S., 335, 336.

Pomponio, 83.

Porsenna, 176.

Portoreale, 241.

Predari, 334.

Priamo, 193, 194, 293.

Proclo, 14.

Publilio Filone, 156, 215.

Puffendorf, 47, 77, 89, 90, 91, 94,95 .

$\mathbf{R}$

Racine, 244.

Ricardo: r. Deschamps.

Rinaldi o cantastorie, 200 .

Rodigino Celio, 203.

Rogadei G. D., 319.

Romano D., 307, 316, 319.

Romolo, 104, 129, 169, 185, 203, $208,227,228$.

Rosa (de) C. A. vedi Villarosa.

Rosmini, 325.

Rossi G., 339.

$\mathbf{S}$

Saint-Evremond, 161.

Salfi, 321, 322.

Sanchez F. (il Brocense), 4, 47, 53 .

Sanctis (de) F., 256, 299, 325, 338 .

Sangro (di) C., 290, 291.

Sangro (di) R., 312.

Sarehi, 336.

Saturno, 167, 187, 213.

Savigny, 256, 323.

Sealigero, 47, 53, 157.

Scevola (Q. Muzio), 176, 296. 
Schelling, 25:.

Schopenhauer, 254.

Schopp G., 47.

Schwarz J., 90.

Scipione Africano, 92, 130, 137.

Scipione Nasica, 215.

Scoto (Duns). 16, 61.

Scrocea A., 340.

Selden G., 47, 77, 94, 154.

Seneca, 163.

Senofonte, 165.

Servio Tullio, 188, 209, 214.

Shakespeare, 235, 244.

Silla, 218.

Simone il giusto, 94 .

Sisifo, 187.

Socrate, 27, 70, 76, 92, 201.

Solla F., 304, 305, 322.

Solone, 156, 188, 212.

Sorel G., 225, 256, 327, 339.

Sostegni, 310.

Spaventa B., 14!, 325, 338.

Spinoza. 26, 80, 81, 89, 103, 143, $205,325$.

Steuco A., 143.

Stobeo. 246.

Stoici, 99, 120 .

Strabone, 197.

Stuart Mill, 324.

Suida, 246.

\section{T}

'Tacito, $76,103,104,151,177,180$, $184,219$.

Tantalo, 187.

Tanueci B.. 316.

Tarquinio Prisco, 188.

Tarquinio Superbo, 52, 175, 209.

Teofrasto, 204.

Terenzio, 168.

Terrasson, 316 .

Tesauro E., 54.

Teseo, 187.
Thierry A., 171, 256.

Tiberio, 14, 130.

Tomasio C., 77, 101, 253.

Tommaseo N., 299, 325, 335 .

Tommaso d'Aquino, 28.

Trabalza C., 340 .

Triboniano, 219, 236.

Trivulzi Cristina. principessa di Belgioioso, 336.

Troya C., 256.

Tucidide, 165 .

Tullo Ostilio, 188.

Turpino, 233.

\section{V}

Vacherot, 324.

Valletta G., 245.

Varrone, 169, 203, 212, 217, 296.

Venere, 168, 169, 187, 191.

Vico Gennaro, 293, 334.

Vico Luisa, 293.

Villarosa, 334, 337.

Virgilio, 203.

Visconti, 171.

Vitry (de), padre, 291, 332.

Voss (Vossio), 64, 196.

Vulcano, 167, 187.

\section{W}

Weber W. E., 323, 336.

Werner, 141, 327, 338, 339.

Windelband, 4 n., 327.

Wolf F., 256, 317-8, 338.

Wood, 318.

\section{Z}

Zeno A., 157.

Zenone, 143, 145.

Zoega, 318.

Zoroastro, 164, 187. 


\section{INIICE}

I. La prima forma della gnoseologia vichiana . . , 1

II. La seconda forma della gnoseologia vichiana . : 21

III. La struttura interna della Scienza nuova . . . " 37

IV. La forma fantastica del conoscere (La poesia e il linguaggio) . . . . . . . . . . . . . . " 45

V. La forma semifantastica del conoscere (Il mito e la religione). . . . . . . . . . . . . . . " 63

VI. La coscienza morale. . . . . . . . . . . » Ti

VII. Morale e religione . . . . . . . . . . . .

VIII. Morale e diritto . . . . . . . . . . . . , 97

IX. La storicità del diritto . . . . . . . . . . " 105

X. La Provvidenza . . . . . . . . . . . . 115

XI. I ricorsi . . . . . . . . . . . . . . . 19 . 19

XII. La metafisica . . . . . . . . . . . . . : 139

XIII. Passaggio alla storiografia. - Carattere generale della storiografia vichiana . . . . . . . „ 149

XIV. Nuovi canoni per la storia del tempo oscuro e favoloso . . . . . . . . . . . . . . \& 161

XV. Le società eroiche . . . . . . . . . . , 173

XVI. Omero e la poesia primitira . . . . . . . . " 191

XVII. La storia di Roma e la formazione delle democrazie . . . . . . . . . . . . $\quad 207$ 
XVIII. La barbarie ritornata o il Medioevo . . . . . pag. 223

XIX. Il Vico contro l'indirizzo di cultura dei suoi tempi " 239

$\mathrm{XX}$. Conclusione. - Il Vico e lo svolgimento posteriore del pensiero filosofico e storico . . . , 249

Luoghi del Vico adoprati o richiamati nel corso dell'esposizione . . . . . . . . . . . . 2559

Appendice :

I. Intorno alla vita e al carattere di G. B. Vico . . : 285

II. La fortuna del Vico . . . . . . . . . . . . , 315

III. Cenni bibliografici . . . . . . . . . . . . $\quad$ > 329

Indice dei nomi . . . . . . . . . . . . . . 343 


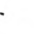



